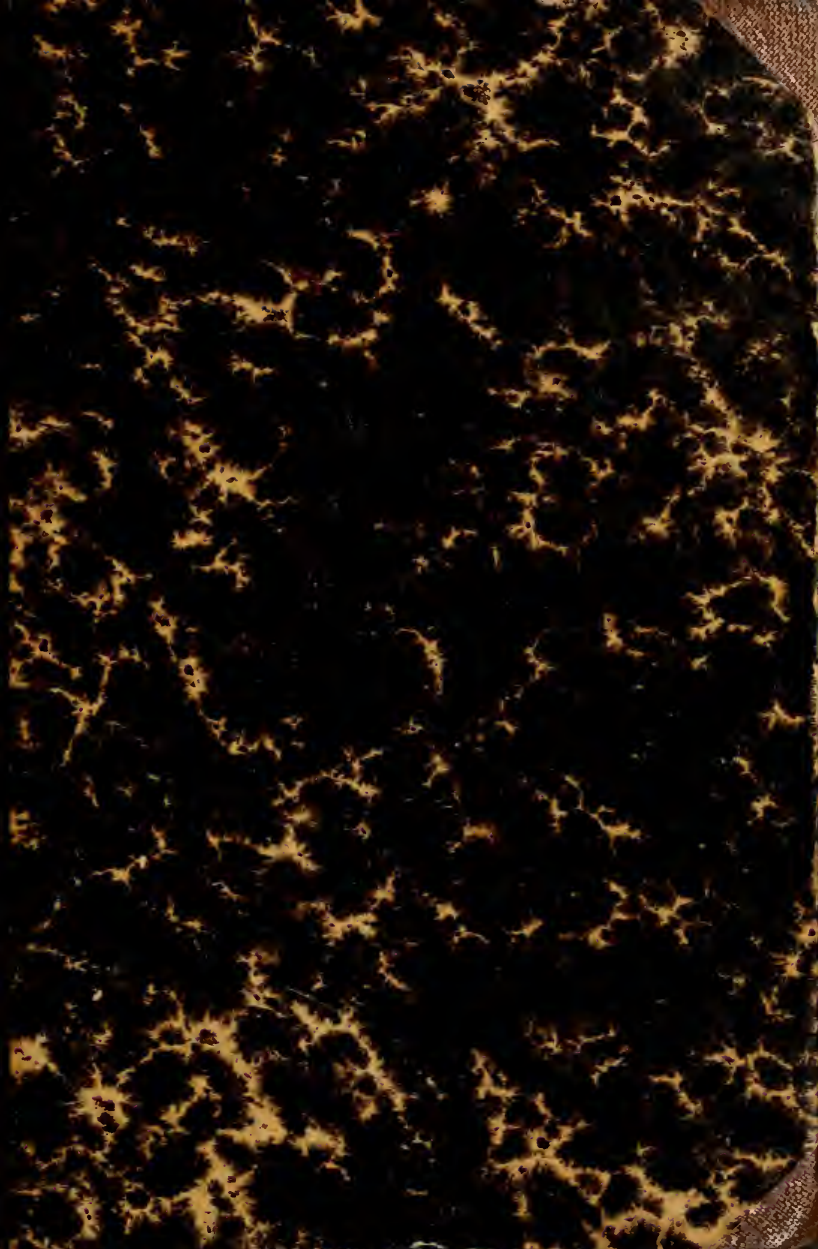




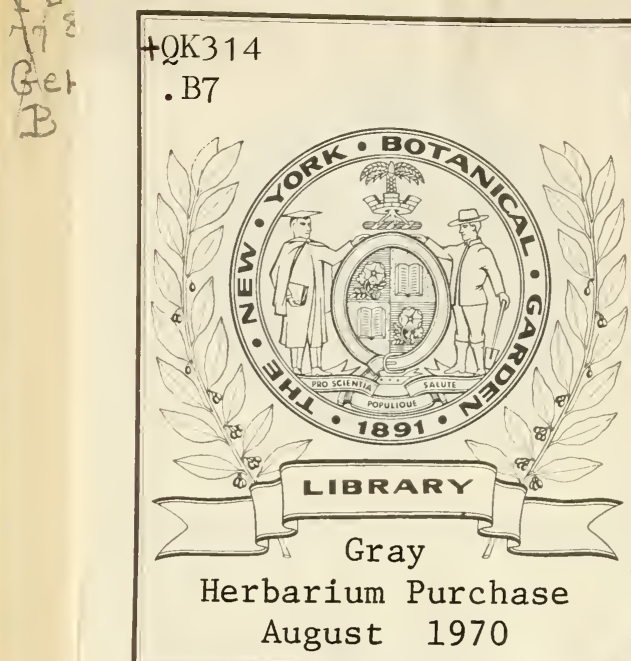

UR $121 \mathrm{E}$

\section{GRAY HERBARIUM}

Received

$$
\begin{aligned}
& \text { Sept. 11, 1933. } \\
& \text { Bought }
\end{aligned}
$$





$$
+0 \times 314
$$


FLORA

B E P O L I N E N I S

SIVE

D E S C R I P T I O

PI AANTARUM PHANEROGAMARUM

CIRle

BEROLINUM SPONTE CRESCENTIUM

VEL IN AGRIS CULTARUII,

A D DITIS

F I I, I I B US ET CHARIS.

BEROLINI,

IN BIBLIOPOLIO FIITTNERIANO. MDCCCXXV.

IATE \& $Y$

NEW $70 \%$

BOTANF(i)

GAROEN 


\section{I R O}

CELEBERRIIIO, ILLUSTRISSIMO, AMPLISSIMO, EXPERIENTTISSINO

C. A SM. RUDOLPH I PHILOS. ET MED. DOCTORI, IUUUS PROF. P. O. MUSEI ANATOMICI DIRECTORI, RECI A CONSIIIS MED. INTIMIS, COLLEGI MEDICO-TECINICI PRIMARIT MEMBRP, EQUITI O. REG. DE AQUILA TIUBA, ACADD. SCIENTIAPUAI BEROLINENS. HOLMIENS. NEAPOIITAN, PARISIENS, PETROjolit, ACAD, NAT, CURIOS. IEOpoldin. MULTARUARUe ALIARUM SOCIETATUM SODAII

PRAECEPTORI AC FAUTORI SUMME VENERANDO. 
E $\mathbf{T}$ 


\section{I $\quad$ R $\quad$ O}

CELEBERRIMO, EXPERIENTISSIMO, AMPLISSIMO, ILLUSTRISSIIIO

\section{Dr. FR. GOTTL. HA YNE}

UNIVEKSTTATIS ITTERARIAE BEROIINENSIS PROFESSORI,

8OCIETATIS AMICORUM NATURAM INDAOANTIUM BEFOLINENSIS, NATURAE CURIOSORUM HAT.ENSIS, IIPsiensis, MARBURoENSIs, PHYTOORAPHICAE ET PHY3ICAE coettingensis, PHARMACEUticae petropolita NAE ET GERMANIAR SEPTENTRIONALIS ATQUE MARCHIAE OECONOMICAE POTSDAMENSIS 8OCIETATIS etc. SODAXI.

PRAECEPTORI ET AVUNCULO

PIE C OXENDO. 


$$
\text { OB COMPLURA }
$$

INSIGLIS BENEVOLENTIAE ET HUMANITATIS

$$
\text { DOCUMENTA }
$$

HAS STUDIORUM PRIMITIAS

D. D. 


\section{S.}

Supervacaneus videtur labor agri Berolinensis plantas describendi, quum eas secundum recentioris Botanices principia descriptas complectatur Opus Cel a Schlechtendal sub titulo: „Flora Mesomarchica et Berolinensis." Sed hic liber, etsi diligentissime doctissimeque in re tractanda versetur, minus commode, ob pretinm voluminisque molem, Botanicem tractare inchoantis plantasque colligendi causa itinera iustituentis usuí patet. Quam ob rem amicis adhortantibus Floram illam Mesomarchicam talem in formam redigeridi cepimus consilium, ut libri pretium vilins fieret et in unum volumen omnes plantae Phanerogamae et eae Cryptogamarum confererentur, quarum cognitio vulgo aestate appeti solet, Filicum scilicet et Chararum. Ipso autem connatu et opere perficiendo occupati in alias discedentes sententias non poteramus, quin, quem ducem nobis esse futurum putavimus, multis locis relinqueremus. 
Hinc factum est, ut et in notis generum proponendis et in agnoscendis et describendis speciebus recederemus, nec etiam omnes notiones terminologicas a recentioribus propositas, quas Schlechtendalius accepit, reciperemus.

Quo facilius ratio et discrimen generum in. telligatur notas genericas fusius tractatas singulis generibus adjecimus et generum notis inter se comcomparatis res primarias in Conspectu posuimus. Quum vero brevitati esset studendum haud exigua signorum et verborum ad compendium collatorum copia libello erat inserenda, quarum, quac majoris sunt momenti, explicare liceat.

Achaen. Achaenium.

A. Altitudo.

Anth. Antherae.

Cal. Calyx.

Caps, Capsula.

Car. Caryopsis.

Cor. Corolla.

DC. De Candolle Regni Vegetabilis Systema.

Filam. Filaneutum.

Fl. Floret.

Flosc. Flosculus.

Fol. Folium.

Fr. Fructus fert.

H. A. Hayne Arzeneigewảchse.

H. F. Hayne dendrologische Flora.

J. Jussieus. 
Inf. s. infer. inferus s, inferior.

Involucr. Involucrum.

Kth. Kunth Flora Berolinensis.

Legum. Legumen.

L. R. S. Linnaei Systema Vegetabilium ed. Roe• mer et Schultes.

L. W. Linnaei Systema Vegetabilium ed. Willdenow.

Mert g. Mertens u. Koch deutsche Flora.

Nect. Nectaritum.

Off. Planta a Medicis adhibita.

Pan. Panicula.

Receptac. Receptaculum.

Schr. g. Schrader Flora germanica.

S. Schlechtendal Flora Mesomarchica et Bero. linensis.

Spermoph. Spermophorum.

Stigm. Stigma.

Stam. Stamina.

Snp. s. super, superus v. superior.

Valv. Valvula.

W. Willdenow. W. p. W. Prodromus.

$1-3^{\prime}$ etc. 1 ad 3 pedes etc.

$1-3$ " etc. 1 ad 3 pollices etc.

4, 5, 6, 7, 8, 9 etc. Menses. 4, Aprilis. 5. Majus etc.

4-6, inde ab Aprili usque ad Majum.

t. Arborem vel etiam flores neutros indicat.

T. Frutex. (Superat altitudinem pedum qua. tuor.) 
7. Fruticulus (Pedes 4 vix attingit).

h Frutesculus. (Rami non lignescunt.)

2 Planta perennis lierbacea.

$\odot$ Planta annua.

$\sigma^{7}$ Planta biennis v. flos masculus,

ఫ̧ Flores hermaphrodití.

우 Flores feminei.

o = Cotyledones accumbentes.

- || Cotyledones inctimbentes.

o $>$ Cotyledones conduplicatae.

Optamus denique, ut arrideat Fortuna laborí et ut leni animo in hoc primo juvenili tentamine nati ex humana imbecillitate perferantur erro. res, quos in posterum, si qua fata sinant, additamentis emendare curae cordique nobis erit.

Valete et favete! Dabam Berolini Cal. Iunii. 


\title{
CONSPECTUS GENERUM.
}

\section{MONANDRIA.}

\author{
1. MIONOGYNIA.
}

1. Hippuris (Hygrobiae Richard) Cal. sup. minimus. Nux.

2. DIGYNIA.

2. Callitriche (Callitrichinae Link) Cor. 2.petala. Sanzarae.

\section{DIANDRIA.}

1. RTONOG INIA.

A) Cal. inf. 4-dentatus. Cur. 1-petala regularis. 3. Ligustrum (O'einae Linh) Bacca. 2-locularis.

4. Syinga (fasmineae f.) Caps. 2-locularis. 13) Cal. inf. rarius. Cor. 1-petala irregularis. $\div$ Caps. polysperma.

5. Veronica (Scroplut'arineae R. Brown) Ca'.4-5. partitus. Cor. subrotata inaequalis.

6. Gratiola Scrophularineae R. Br.) Cal.5-partitus Cor. 2-labiata.

7. Pinguicola (Lentibulariae Rrch.) Cal. 2-Labiatus - 5-fidis. Cor ringens calcurata.

S. Uiricularia (Lentibulariae Rich) Cal, 2-sepalus. Cor. personata v. ringens calcarata. 
+† Achaen. 4 .

9. Lycopus (Labiatae 7.) Cor. tubulosa Stam, di. stantia.

10. Salvia (Labiatae f.) Cor. ringens Stam. trans. verse pedicello affixa,

C) Cal. sup. 2-sepalus Cor. 2-petala.

11) Circaea (Onagrae f.)

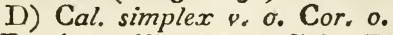

12. Fraxinus (fasmineae f.?, Flor. polygami. Samara.

13 Lemna (Hydrocharidibus aff. $R, B r$. ) Cal. 1phyllus. Caps.

14 Cladium (Cyperoideae f.) Cal.glumaceus multi-valvis. Achaen.

2. DIOYNIA.

15. Anthoxanthum (Gramineae f.) Cal. glumaceus 2-valv. 1-Horus. Cor. duplex.

\section{TRIANDRIA.}

1. MONOGYNSE。

A. Cal. sup. margo. Cor. $5-f i d a$.

16. Valeriana (Valerianeae DC.) Achaen. pappo coronatum.

17. Fedia (Valerianeae DC.) Nux. calyce coronata. B. Cal. o. Cor. sup. 6-partita.

19. Iris (Irideae f.) Corollac laciniae alternatim re. fl. xae.

C. Cal. inf. 5.sepalus. Cor. o. 19. Yolycuemum (Chenopodeae 7.3) Achaen membranaceum. 
D. Cal. glumaceus 1-valy. inferus. Cor. 0. Achaen.

20. Cyperus (Cyperoideae 7.) Glumae distichae imbricatae omnes floriferae.

21. Scirpus (Cyperoideae $f_{\text {.) }}$ Glumae undique imbricatae fere omnes floriferae.

22. Schoenus (Cyperoideae f.) Glumae undique im. bricatae, $3-4$ inferioribus minoribus vacuis. 23. Eriophorum (Cyperoideae 7 .) Glumae undique imbricatae. Setae hypogynae lanae similes excrescentes.

E. Cal. o. Cor. 2-valy, glumacea.

24. Nardus (Gramineae 7.)

\section{DICYNIA.}

\section{A. Cal. o. Cor. 2-valvis.}

25. Leersia (Gramineae 7.)

B. Cal. plerumque 2-valvis. Cor, plerumque 2valvis.

+ Inflorescentia paniculata.

a) Cal. I-florus.

26. Alopecurus (Gramineae 7.) Calycis valv. basi connatae. Cor.1-valv. aristata.

27. Phleum (Gramineae f.) Calycis valv. carinatocompressae. Corollae valv. membranaceae, Rudimentum pedicelliforme v. o.

28. Phalaris (Gramineae 7.) Calycis valv. carinatocompressae. Corollae valv. cartilagineae Rudimenta squamaeformia.

29. Milium (Gramineae f.) Calycis valv. convexoventricosae. Corollae valv. demum cartilagineae caryopsin cingentes. 
30. Agrostis (Gramineae 7.) Calycis valv. convexo. compressae nutuicae. Corollae valv. membrana. ceae, basi pilis brevissinis instructae.

31. Arundo (Gramineae f.) Calycis valv. convexocompressae. Corollae valvulae membranaceae, basi pilis quartam corolla e partem aequantibus $v$. longiorib. cinctae. Rudimentum pedicelliforme. 32. Stipa (Gramineae f.) Calycis valvulae convexocompressae Corollae (Flosculi) nervo medio apice incrassato et in aristam elongato.

b) Cal. multiflorus. «. Flosculis omnibus

33. Avena (Gramineae f.) Cal. 2 -multillorus. Corcllae valv. infer. apice dentata 2 -fida v. 2 aristata, arista tortili sub v. supra medium dorsum oriente.

34. Triodia (Gramineae f.) Cal. 3-5-Alorus, valv. magnis convexo-dilatatis flosculos cingentibus. Cor. apice 3-dentata, dente medio in mucronem protracto.

35. Bromus (Gramineae f) Cal. 3. multiflorus. Corollae valv. sup. in carina ciliis rigidis pectinata, infer. apice emarginata 2-mucronata v. 2 aristata, arista subapicali.

36. Festuca (Gramineae f.) Cal. 2-multiflorus flosculis brevior. Flosc. lanceolati v. lanceolato-subulati, dorso rotundato. Spiculae ebracteatae. 37. Aira (Gramineae f.) Cal. 2-florus v. 2-florus c. rudimento flosculi 3. Corollae valv. infer. aristata, arista recta e basi v. medio dorso oriente, 38. Koeleria (Gramineae 7.) Cal. 2-5-florus, carinato-compressus. Flosc. recti sublanceolati cari- 
nato-compressi. Cor. valv. inf. mutica mucro. nata v. sub apice aristata.

39. Donax (Gramineae f.) Cal. valv. lanceolatae. Corollae valv. lanceolatae dorso rotundatae basi barbatae, inferior apice 3-5-cuspidata. 40. Dactylis (Gramineae f.) Calycis valv. mem. branaceae compressae. Flosc. lanceolati apice in. curvati, dorso carinato-compressi. 41. Cymosurus (Gramineae f.) Cal. 3-5-florus. Flosculi Festucae Bracteae e valvulis (glumis) distichis compositae spiculas cingentes. 42. Briza (Graminae f.) Cal flosculis brevior. Co. rollae valv. ventricosae basi cordato-auriculatae. 43. Glyceria (Gramineae 7.) Cal. convexus. Flosc. ob.

longi obunsi intus subventricosi, dorso roundato. 44. Poa (Gramineac 7.) Cal. compressus. Flosc. lanceolativ. ovati recti, dorso carinato.compressi. 45. Molinia (Gramineae f.) Cal. convexus. Flo. sculi latere interiore supra basin ventricosi dein conici, dorso recto rotundato.

3. Flosculis aliis 호, allis 南 et $\sigma^{7}$. 46. Melica (Gramineae 7. ) Calycis valv. ovatae magnae. Flosc inferiores $\not$, superiores $\hbar$. 47. Phragnites (Gramineae f.) Calycis valv. lanceolatae acuminatae floscul. breviores. Flosc. in. fimus th $\mathrm{v}$. ${ }^{\top}$ sessilis, reliqui pedicellis sericeovillosis insidentes ఫૃ.

48. Panicum (Gramineae f.) Calycis valv. inferior. parva $\nabla$. o. Flosc. superior $\Varangle$; inferior $\sigma^{\top} \mathrm{v} . \frac{\hbar}{7}$, valv. superiore minore v. o. 49. Arrhenaterum (Gramineae $\mathfrak{F}$.) Calycis valvulae ovato.lanceolatae membranaceae. Flosculus infe. 
ferior $\sigma$, valvula inferiore infra medium aristata, superior $\not$.

50. Holcus (Gramineae f.) Calicis valvulae Hosculis longiores. Flosc. super. ơ.dorso aristatus, inferior $\not$ muticus. Vaiv. conıpressae lanceolatae herbaceo-membranaceae.

51. Hierochloe (Gramineae f.) Calicis valv. flosculos aequantes Flosc. 2 inferiores $\sigma^{\top}$, summus (medius) જ 2 -andrus. Valv, convexo-compressae ovatae. + + Inflorescentia spicata.

a) Spicula solitaria in quavis rhacheos excisura.

52. Lolium (Gramineae f.) Cal. 1-valvis 3-multiHorus. Spiculae latere dorsali receptae. 53. Brachypodium (Gramineae f.) Cal. multiflorus. Curollae valv. inf. in aristam rectam elougata v. subulata.

54. Triticum (Gramineae f.) Cal. 2-valvis 3-multiflorus, valv. lanceolat. v. compresso-venticicosis carinatis. Cor aristata v. mutica.

55. Secale (Gramineae f.) Cal. 2-florus cum rudimento 3 flosculi pedicellato, valvulis subulatis. Corollae valv. infer. in setam subulata, superior casinata.

b) Spiculis $2 \%$ plurib. in quasis rhacheos excisura.

56. Elymus (Gramineae f.) Cal. 2-multillorus, valv. angus:e lanceolat. $v$. subulat. ante spiculas positis. Flosc. ఫ̧.

57. Hordeum (Gramineae 7.) Cal. 1-florus ante flosculos positus, valv. lanceolatis $v$. subulatis. Flosc. medius $\not$, laterales $\sigma^{\pi}$ v. th $\nabla$. 


\section{TRIGYNIA.}

58. Montia (Portulaceae f.) Cal. 2-sepalus. Cor. 1-petala irregularis.

59 Holosteum (Caryophylleae f.) Cal. 5-sepalus.

Cor. s-petala.

Stellaria media. Arenaria viscidula.

\section{TETRANDRIA,}

1. MONOCYNIA.

* Floribus superis.

A. Cor. 1-petata.

$\div$ Achaen. simplix pappo coronatum. Inso. lucr. polypliyllum.

6o. Dipsacus (Dipsaceae f.) Receptac. elongatum paleaceum. Papp. integer. Cor. 4-fida. 61. Scabiosa (Dipsaceae F.) Receptac. paleaceum ro. pilosum. Papp. varie fissus. Cor. 4-5-fida. t+ Achaen. duplex. Cal. margo obsoletus 4-5-dentatus.

a Cal. persistens achaenia coronans. 62. Scherardia (Rubiaceae f.) Cor. infundibuliformis.

b) Cal. deciduus.

63. Asperula (Rubiaceae F.) Cor.tubuloso-infundibuliformis.

64. Galium (Rubiaceae f.) Cor rotata, tubo brevissimo.

B. Cor. 4-petala. 65. Cornus (Caprifoliaceae f.) Cal. 4-dentatus Drupa.

- B. $F$. ** 
66. Trapa (Hydrocaryes Link) Cal. 4-partitus. Nux. spinosa.

* Eponymus europaeus. Floribus inferis. A. Cal. et cor. Caps.

67. Plantago (Plantagineae f.) Cor. hypocrateriformis. Stam. longissima.

68. Centunculus (Primulaceae f.) Cor. tubulosa. Stam brevia.

B. Cal. simplex. Cor. o. Achaen. 69. Sanguisorba (Rosaceae f.) Cal. 4-sepalus, sepalis receptaculo insertis.

70. Alchemilla (Rosaceae f.) Cal. 8-fidus, laciniis altern. minoribus.

71. Parietaria (Urticeae 7.) Polygama. Cal. 4-fidus. Convallaria bifolia.

2. TETRAGINIA.

72. Potamogeton (Potamophilae Rich.) Cor. 0. Achaen 4.

73. Sagina (Caryophylleae 7.) Cor. 4-petala. Caps, Linum Radiola.

V. PENTANDRIA.

1. MONOGYNIA.

- Cal. et cor.

+ Floribus inferis.

A. Achaen. 4 .

a) Faux corollae fornicibus clausa.

74. Myosotis (Boragineae f.) Cor. hypocrateriformis, fornicibus (squamis) brevibus. Achnen. calyci affixa.

75. Lappula (Boragineae 7.) Cor. hy pocrateriformis, fornicib.brevibus. Achaen. columnaria, echinata. 
76. Cynoglossum (Boragineae f.) Cor. infundibu. lifornis, fornicib. erectis. Achaen. columnaria. 77. Asperugo (Boragineae f.) Cor. subinfundibu. lifornis. Achaen. columnaria. 78. Anchusa (Boragineae f.) Cor. infundibulifor. mis, fornicibus erectis obtusis. Achaen. calyci affixa.

81. Symphytum (Boragineae f.) Cor. cylindricocampanulata, fornicibus in conum comiventibus. b) Faux corollae non clalisa.

79. Pulmonaria (Boragineae f.) Cor. infundibuliformis, fauce pilosa. Achaen. mrbinata. 80. Lithospermum (Boragineae f) Cor. infundibuliformis, fauce nuda. Achaen. ovata.

82. Echium (Boragineare $f$.) Cor, infundibuliformis v. canpanulata, fauce anspliata nuda. Achaen. turbinata.

B. Caps.

83. Anagallis (Primulaceae Vent.) Cal. 5-partitus. Cor. rotata. Caps. circumscissa.

84. Primula (Primulaceae Vent.) Cor. Hypocrateriformis, tubo cylindrico. Caps, 10.dentata apice dehiscens.

85. IIottonia (Primulaceae Vent.) Cor. hypocrateriformis. Caps. evalvis.

86. Lysimachia (Primulaceae f. Cor rotata. Stam. basi dilatata v. connata Caps. 5-valyis. 87: Menyanthes (Gentianeis affine R. Brown) Cor. infundibuliformis, intus villosa. Stigm. 2-lobum. Caps. 2-valvis.

88. Erythraea (Gentianeae 7.) Cor. infundibuliformis. Anth. demum spirales. Caps. 2-valvis, 
89. Viola (fonideae Sprengel) Cor.irregularis 5-petala corniculata, Anth. coliaerentes. Caps. 3. valvis.

go. Impatiens (Geraniis aff: f.) Cal. 2-sepalus. Cor. 5-petala irregularis, Caps. 5-valvis. 91. Evonymus Rhamneae 7.) Cor. 5-petala. Caps. 5-gona, 5-valvis (Numerus interdum 4-narius). 92. Verbascum (Solaneae f.) Cor. rotata. Filam. barbata. Caps, 2-valvis.

93. Convolvulus (Convolvulaceae . F.) Cor. campanulata plicata. Caps. 2-3-valvis.

94: Hyoscyamus (Solaneae f.) Cal. persistens. Cor. infundibuliformis. Stam, inclinata. Caps, operculata.

95. Datura (Solaneae 7 ) Cal. angulatus tubulosus deciduus. Cor. infundibuliformis plicata. Caps. 4-valvis.

C. Bacca.

96. Solanum (Solaneae 7.) Cor. rotata, interdum plicata. Anth. conniventes.

97. Lycium (Solaneae f.) Cor. infundibuliformis. Stam. basi villosa.

9.9. Rhamnus (Rhamneae f.) Cal. campanulatus. Cor. 5.petala. - E. Folliculus.

99. Vinca (Apocyneae f.) Cor. hypocrateriformis, fauce nuda. $\dagger+$ Floribus superis. A. Caps.

10o. Jasione (Campanulaceae f.) Cor, rotata profunde 5-partita. Antherae cohaerentes. Caps. 2-lo. cularis apice pervia. 
101. Phyteuma (Campanulaceae 7.) Cor. rotata 5. partita. Caps. $2-3$-locularis lateraliter dehiscens. 102. Campanula (Campanulaceae 7 ) Cor. campanuiata. Stam. basi dilatata fornicata. Caps. 3-Iocularis lateraliter deliiscens.

B. Bacca.

103. Ribes (Grossulariae DC.) Cor. 5-petala calyci

inserta.

104. Lonicera (Cqprifoliaceae f.) Cor. 1-petala curvatu-tubulosa irregulariter 5 -fida. 105. Hedera (Caprifoliaceae 7.) Cor. 5-petala. Bacca sicca.

$$
\text { * Cal. simplex. Cor o. }
$$

106. Illecebrum (Paronychiae St. Hilaire). Cal. persistens. Filam. basi connata. Caps. 107. Thesium ((Santalaceae R. Brown.) Cal. campanulatus, laciniis basi barbatis. Achaen.

Polygonum minus, amphibium.

\section{DIOYNIA. \\ * Cal. simplex. Cor. c.}

108. Ulmus (Urticeae f.) Cal. 4-5-dentatus. Samara.

109. SaIsola (Chenopodeae DC.) Achaen. calyce mutato inclusum:

110. Chenopodium (Chenopodeae DC.) Achaen. membranaceum calyce immutato tectum 111. Atriplex (Chenopodeae DC.) Polygamum. Cal 꼬 5-sepalus, $\bigcirc$ 2-sepalus. Achaen: nembranaceum calyce tecium

112. Hemiaria (Amaranthaceae 7.). Squamulae 5 cum calycis laciniis alternantes, Achaen. * Cal. et cor. 


\section{A. Caps.}

113. Cuscuta (Convolvulaceis aff. f.) Cor. urceolato.campanulata.

114 Gentiana (Gentianeae f.) Cor. 1-petala in. ferne tubulosa.

B. Folliculus.

115. Cymanchum (Apocineae 7.) Cor. subrotata 5-partita. Nect. corona duplex.

C. Achaen 2.

a) Umbellae subcapitatae.

116. Eryngium (Umbelliferae $\mathcal{f}$ ) Involucr. subspinosa. Fruct. paleaceo-squamati.

117. Sanicula (Umbelliferae f.) Involucr. oligophylla. Fruct. oblongi setis obsiti.

b) Unbellae imperfectae, involucr. obsolet. $v$. o. (Fol. subsimplicia).

118. Hrdrocotyle (Umbelliferae f.) Fruct. subrotundi compressi, dorso tricostato.

c) Umbellae perfectiores. Involucra latiuscula. (Fol. simplicia).

418. Bupleurum (Umbelliferae 7.) Fruct. ovatis. prismatici solidi, cost. 5. obtusiusculis, suturib (raphe) excavata.

d) Umbellae perfectae interdum evolucratae. Fruct. solidi 5-costati. (Fol. composita).

119. Pimpinella (Umbelliferae f.) Involicra 0. Fruct. ovati costis obtusiusculis, valleculis pla. niusculis vittatis.

120.. Seseli (Umbelliferae f.) Involucr, universale o, sed partiale. Fruct. ovati, costis acutis, valleculis sulcat. striatis.

121. Sison (Umbelliferae f.) Involucra oligophylla 
ซ. o. Frtict. ovatus, costatus, valleculis convexis,

Jatusculis contractis sutura (raphe) excavata.

123. Carum (Umbelliferae f) Involucr. 1-phyllum.

Involucellat o. Fruct. oblongo-prismaticus, costis

3 dorsalibus obtusiusculis, valleculis sulcat. stria.

tis, sutura (raphe) lata utrinque costata.

123. Cnidium (Úmbelliferae f.) Involiser. sub o. $\nabla$.

1-plyyllum. Fruct. ovatus, costis acut. subulatis,

$\nabla$ allecul. sulcatis striatis.

124. Ocnanthe (Umbelliferae 7.) Involucr. o. Involucella polyphylla. Fruct. prismatici, costis acu. is $v$. obtusis, apice calyce pistillisque coronati. 125. A pium (Umbelliferae 7.) Involucr. et in volucella o. Fruct. ovati, costis obtusiusculis, valleculis suturaque planis.

126. Meum (Umbelliferae f.) Involucr. o. Involucella oligophyila. Fruct. prismatici, costis acutis, vallecul. planis, sutura excavata.

e) Umbellae perfectae. Involucra plerumque deficientia. Fructus corticati solidi v. compressi utriculati.

127. Cicuta (Umbelliferae 7.) Involucr. subnullum. Fruct. ovati solidi 5 -costati, cortice in valleculis prominulo.

128. Aethusa (Umbelliferae 7.) Involucr. o. Involucella dimidiata. Fruct. ovati solidi, cortex in costas 5 acutas turgescens.

129. Thysselinum (Umbelliferae f.) Involucra et involucella. Fruct. plani, cortice in dorso $3 \mathrm{co-}$ ctas obtusas exlibente, sutura plana. 
f) Involucra oligo- v. polyphylla. Fructus armati hirti.

130. Daucus (Umbelliferae f.) Involucr. pinnatum. Flor. subradiantes. Fruct. oblongi, costis 5 aculeatis, angulis interpositis setosis.

131. Torilis Umbelliferae f.) Involucr. obsoletum. Flores aequales. Fructus ovati costati undique setis aculeisve tecti.

g) Involucrum o. v. subnullum. Fructus pyramidati rostrati.

132. Scandix (Umbelliferae f.) Involucr. o. v. ejus loco folium caulinum. Fruct. pyramidat, rostrat. 5-costatus.

133. Myrrhis (Umbelliferae 7.) Involucr. o. Fruct. pyramidatus rostratus, costis 5 acutis, pistillis. coronatus.

134. Chaerophyllum (Umbelliferae f.) Involucr. subnullum. Fruct. conicus evittatus ecostatus gla. ber, sutura sulcata.

135. Anthriscus (Umbelliferae f.) Involucr. subnullum. Fruct. conici muricati, pistillis persistentibus, sutura sulcata.

h) Involucra et involucella varia. Fiructus sub. orati costati sulcatio.

136. Sium (Umbelliferae 7.) Involucra integra: Fruct. subprismaticus, costis 5 obtusis, vallecu. lis subconvexis.

137. Conium (Umbelliferae f.) Involucra et invo. lucella. Fruct. ovati, costis 5 obtusis, immatur. crenulatis, vallecul. planis. 138. Ligusticum (Umbelliferae f.) Involucra et in- 
volucella varia. Fruct. ovato-oblongus, costis 5 acutis, vallecul. sulcatis.

i) Involucra varia. Fructus compressi plani saepe alati.

139. Selinum (Umbelliferae 7 .) Involucra et involucella. Fruct: planissimi ovales, margine alati, costis dorsalibus 3 subproninulis, valleculis suturaque vittatis

140: Heracleum (Umbelliferae 7.) Involucr. o. Flor: subradiantes. Fruct. compressi membranaceo-matginati, costis 3 dorsalib. obtusis, vallecularum et commissurae (suturalis) vittis clavatis. 141. Pastinaca (Umbelliferae f.) Involucr. n, involucella subnulla. Fruct. ovalis compressus planus marginatus, costis dorsalibr:s obsoletis. 142. Angelica (Umbelliferae 7) Involucr. o. Fruct. subcompressus, costis 3 acut. alatis, valleculis sulcatis margine membranaceo. 143. Laserpitium (Umbelliferae J.) Involucra et involucella polyphylla. Fruct. ovalis subcompressus, costis 3 primariis acutis, secundariis alatis.

\section{TRICYNIA. \\ * Floribus superis}

144. Viburnum (Caprifoliaceae 7.) Cor. 5-fida.

Bacca 1-sperma.

145: Sambucus (Caprifoliaceae f.) Cor. rotata 5-fida. Bacca 3-sperma.

* Floribus inferis.

146. Corrigiala (Portulaceae f.) Cor. 5-petala. Achaen. 
147. Drosera (Droseraceae DC.) Cor. 5-petala. Caps. 1-locularís.

Stellaria media.

4. TETRAGYNIA.

148. Parnassia (Resedaceae DC.) Cor. 5-petala, Nect. squamae 5 globuliferae. Caps.

5. Pentagria.

149. Armeria (Plumbagineae $\tilde{J}$.) Involucr. polyphyllum Cal. 1-phyllus, limbo scarioso. Cor. infundibiliformis. Achaen.

150. Linum (Linoideae DC.) Cal. 4-5-sepalus. Cor. 4-5-petala. Caps.

Cerastium semidecandrum. Spergula arvensis, pentandra.

PENTANDRIA POLYGria. Ranunculus Myosurus.

VI. HEXA N D R I.

\section{MONOG YNIA. \\ * Bacca.}

151. Berberis (Berberideae f.) Cal. 6-sepalus. Cor. 6-petala.

152 Acorus (Aroideae f.) Spadix. Cal. o. Cor. 6. petala.

153. Asparagus (Asphodeleae R. Brown.) Cal.o. Cor. 6-partita, petalis 3 inferioribus apice reflexis. 154. Convallaria (Smilaceae R. Brown.) Cal.o. Cor. 1-petala 4-6-fida.

$$
\text { * * Caps. }
$$

155. Anthericum (Asphodeleae R. Brown.) Cal. o.

Cor. 6-petala patens. Filam. filiformia. 
156. Ornithogalum (Asphodeleae R. Brown.) Cal. o. Cor. 6.petala persistens. Filam. basi dilatata.

157. Lilium (Liliaceae f.) Cal. o. Cor. 6-petala. Nect. sulcus longitudinalis versus basin petalorum.

158. Allium (Asphodeleae R. Brown.) Spatha arida. Cal. o. Cor. 6.petala.

159. Juncus (Funceae f.) Cal. o. Cor. 6-petala. Stigm. 3. Caps. 3-locularis. (Fol. teretia)

16o. Luzula (funceae f.) Cal. o. Cor. 6.petala. Caps. 1-locularis. (Fol. plana.)

161. Peplis (Salicariae f.) Cal. 12-dentatus. Cor. 6-petala v. o.

Polygonum Hydropiper, Persicaria.

2. TRICYNIA.

* Caps.

162. Schenchzeria (Alismaceae R. Brown.) Cal. 6-sepalus. Cor. o.

163. Triglochin (funcagineae Rich.) Cal. 3-sepalus.

Cor. 3-petala.

164. Colchicum (Melanthaceae R. Brown.) Cal. o.

Cor. infundibiliformis radicalis.

165. Tofieldia (Melanthaceae R. Brown.) Cal. 3-fidus. Cor. 6-petala.

$$
\text { **Achaen. }
$$

166. Rumex ((Polygoneae f.) Cal. 3-phyllus. Cor. 3-petala. Achaen. 1.

\section{POIYGYNIA.}

167. Alisma (Alismaceae R. Brown) Cal. 3-fidus per. sistens. Cor. 3-petala. Achaen. plura. 


\section{HEPTANDRIA.}

168. Aesculus (Hippocastaneae DC.) Cal. 1-phyllus. Cor. 4-5-petala. Caps.

169. Trientalis (Primulaceae Vent.) Cal. 7-sepalus. Cor. 7-petala. Caps

170. Calla (Aroideae f.) Spatha 1-phylla. Spadix. Bacca.

\section{OCTANDRIA.}

1. MONOG YNIA.

- Floribus superis.

171. Oenothera (Onagreae f.) Cor. 4-petala. Caps. Sem. libera.

172. Epilobium (Onagreae f.) Cor. 4-petala. Caps. Sem. desmate instructa.

173. Vaccinium (Ercaceae f.) Cor. 1-petala urceolata $\nabla$. campanulata. Bacca.

174. Oxycoccos (Ericaciae f.) Cor. 1-petala, petalis reflexis. Bacca.

$$
\text { * * Floribus inferis. }
$$

175. Calluna (Ericaceae f.) Cor. duplex. Caps. 176. Erica (Ericaceae F.) Cor. 4 -fida simplex. Caps. 17. Accr (Aceraceae F.) Flor. polygami. Cor. 5-petala v. o. Samarae.

2. TRICYNIA.

178. Polygonum (Polygoneae 7.) Cal. o. Cor. 5-partita persistens. Achaen. angulosum.

3. TETR AG YNIA.

179. Paris (Smilaceae R. Brown) Cal. 4-sepalus. Cor. 4-petala v. o. Bacca 4-locularis. 
180. Adoxa (Saxifrageae 7.) Cal. 2-4-dentatus, basi cum germine comnatus. Cor. 4-5-fida. Bacca calyce semivestita 1-locularis.

191. Elatine (Caryophylleae 7.) Cal. 4-sepalus. Cor. 4-petala. Caps.

\section{ENNEANDRIA.}

1. HEXAgYNia.

1\$2. Butomus (Butomeae Richard) Cor.6-petala, Caps.

\section{DECANDRIA.}

1. MONOGYNIA.

* Floribus polypetalis.

183. Monotropa (Monotropeae?) Cal. 4-5-sepalus. Cor. 4-5-petala, petalis basi excavatis melliferis. Caps.

ISt. Ledum (Rhododendraceae 7.) Cal. 5-fidus. Cor. 5-petala expansa. Caps. 5 spermophoris apice ad. natis.

185. Pyrola (Ericaceae f.) Cal. 5-partitus. Cor. 5petala. Caps. spermophoris 5 centralibus. * Floribus 1-petalis.

186. Andromeda (Ericaceae f.) Cal.5-partitus. Cor. ore 5-fido. Caps.

157. Arbutus (Ericaceae 7.) Cal. 5-partitus, Cor. 5-fida, basi diaphana. Bacca.

2. DICYNIA.

* Cal. simplici, cor. 0.

188. Chrysosplenium (Saxifrageae f.) Cal. 4-5. partitus (coloratus), Caps. 
190. Scleranthus (Caryophylleae 7.) Cal. 5-denta. tus. Achaen.

* Cal. et cor.

189. Saxifraga (Saxifrageae 7 .) Cal. 5-partitus. Cor. 5-petala. Cáps. birostris inter rostra dehiscens.

191. Gypsophiła (Caryophylleae 7.) Cal. 1-sepalus. 5-gonus 5-dentatus. Cor. 5-petala, petalis unguiculatis. Cáps. globosa.

192. Saponaria (Caryopylleae f.) Cal. 1-sepalus tt. bulosus. Cor. 5-petala, petalis nnguiculatis ad basin laminae 2-dentatis. Caps. oblonga.

193. Dianthus (Caryophylleae f.) Cal 1-sepalus tubulosus calyculatus. Cor. 5-petala, petalis unguiculatis. Caps. cylindrica.

\section{TRIOINIA. \\ * Cal. I-sepalo.}

194. Cucubalus (Caryophylleae f.) Cor. 5-petala, Nect. 0.

95. Silene (Caryophylleae 7.) Cor. 5-petala. Nect. corona ad faucem.

$$
\text { * Cal. 5-sepalo. }
$$

196. Stellaria (Caryophylleae f) Cor. 5-petala, pe. talis bipartitis.

197. Arenaria (Carophylleae f.) Cor. 5-petala, pe. talis integris.

4. PENTAGYNia.

198. Spergula (Caryophylleae f.) Cor. 5-petala, petalis integris. 
199. Cerastium (Caryophylleae f.) Cor. 5-petala, petalis bifidis. Nect. o. 200. Lychnis (Caryophylleae f) Cal. 1-sepalus. Cor. 5-petala, petalis bifidis. Nect. corona. 201 Agrostemma (Caryophylleae f.) Cal. 1-sepalus 5-gonus. Cor. 5-petala, petalis integris. Nect. o. 202. Sedum (Crassulaceae DC.) Cal. 5-i2-fidus. Cor. 5-12-petala. Nect squamae 5. 203. Oxalis (Oxalideae IC.) Cal. 5-sepalus. Cor. 5 -petala, petalis unguribus connexis. Stam. inaequalia, filamentis basi connatis.

\section{ICOSANDRIA.}

I. MONOGYNIA,

* Floribus superis.

30.4. Asarum (Asarinae Kth.) Cal. 3-4-fidus. Cal. o. Caps. calyce coronata. * * Flortbus inferis.

20.5. Lythrum (Salicariae f.) Cal. 1-sepalus. Cor. 6-petala. Caps. 206. Prunns (Rosaceae 7.) Cal. 5-fidus. Cor. 5-petala. Drupa.

2. DIG YNIA.

207. Agrimonia (Rosaceae f.) Cal, 1-sepalus, Cor. 5-petala. Achaen. 2.

3. TRIGYNIA.

208. Reseda (Resedaceae DC.) Cal. 1.sepalus. Cor. 4-6-petala, petalis laciniatis. Caps.

Pyrus Aucaparia. 
4. PENTAOYNiA.

2ng. Pyrus (Rosaceae 7.) Cor. 5.petala. Pomum. 210. Mespilus (Rosaceue 7.) Cor. 5-petala. Bacca. 211. Spiraea (Rosaceae 7.) Cor. 5-petala. Caps.

5. POIYGXNIA.

212. Rosa (Rosaceae 7.) Cal. 5-partitus. Cor. 5-petala. Achaenia a receptaculo carnoso urceolato recepta.

213. Rubus (Rosaceae 7.) Cal. 5-fidus. Cor. 5-p̈etala. Bacca composita

214. Fragaria (Rosaceae 7.) Cal. 10-fidus. Cor. 5-petala. Achaen. carpophoro carnoso inserta. 215. Potentilla (Rosaceae 7 ) Cal. 8-10-fidus. Cor. 4-5-petala. Achaen. receptaculo exsucco imposita. 236. Comarum (Rosaceae 7.) Cal. Io-fidus. Cor 5tala, petalis calyce minoribus. Achaen. receptaculo spongioso inserta.

217. Geum (Rosaceae 7.) Cal. io-fidus. Cor. 5-petala. Achaen. aristata receptaculo columnari imposita.

\section{POLYANDRIA.}

1. MONOGXNIA.

A. Cal. 3-sepalo deciduo, cor. 4-petala. 218. Chelidonium (Papaveraceae 7.) Caps. siliqui. formis.

219. Papaver (Papaveraceae 7.) Caps. suburceolata. B. Cal. polysepalo, cor. polypetala.

220. Nymphaea (Nymphaeaceae Salisb.) Cal. 4-5sepalus. Nect. glandula in medio Stigmate. 
221. Nenuphar (Nymphaeaceae Salib.) Cal. 5-6-8epalus Nect. dorsum petalorum.

C) Cal. 5-partito v. 5-sepalo, cor. 5-petala. 222. Tilia (Tiliaceae f.) Caps, drupacea 5-locularis.

223. Helianthemum (Cisticiae f.) Calycis sepala duo minora. Caps. 1-locularis.

2. TRIGYNIA.

224. Euphorbia (Euphorbiaceae f.) Cal. 1-sepalus ventricosus. Cor. 4-5-petala. Germen pedicellatum. Caps. tricocca. 225. Delplinium (Ranunculaceae f.) Cal. o. Cor: 5-petala calcarata. Nect. 1-4-phyllum calcara tum. Caps. 1-valvis.

3. pentagyia.

226. Aquilegia (Ranunculaceae f.) Cal. o. Cor. 5petala. Nect. corona 5-phylla, loliol. calcaratis. 227. Nigella (Ranunculaceae f.) Cal. o. Cor. 5-petala. Nect. 5, \& v. plura 2-labiata 2-glandulosa

\section{POLYGYEIA.}

228. Thalictrum (Ranuncu'aceae 7.) Cal, o. Cor. 4-5-petala. Achaen. ecaudati. 229. Pulsatilla (Ranunculaceae F.) Cal. o. Cor. 6-petala. Achaen. caudata.

23\%. Anemone (Ranunculaceae f.) Cal. o. Cor. 69-petala. Achaen. ecaudata. 231. Adonis (Ranunculaceae f) Cal.5-sepalus. Cor.

5-15-petala, petalis ungue nudis. Achaen. 232. Ranunculus (Ranunculaceae $f$.) Cal. 3-8-seF. B. *** 
palns. Corollae petala ungne, poro v. squama nectarifera instructa. Achaen.

233. Caltha (Ranunculaceae f) Cal. o. Cor. 5-petala. Caps. $4-5$.

234. Trollius (Ranuculaceae f.) Cal. o. Cor. polypetala. Nect. corona polyphylla, foliolis basi tubulosis. Caps.

\section{DIDYNAMIA.}

1. TOMOGYNIA.

* Cal. plerumque 5-fidi v. 5-dentati. Achaen. 4.

235. Ajuga (Labiatae f.) Coroltae labium superius minimum bidentatum.

236. Tencrium (Labiatue 7.) Corollae Iabii supe-

rioris loco incisura, in qua stamina jacent.

237: Nepeta (Labiatae f.) Corollae faux dilatata maxgine rellexa, labio super erecto emarginato, in-

fer. subrotundo crenulato.

238. Mentha (Labiatae f.) Cor. subaequal. 4-fida,

lacinia super. latiore emarginata. Stam. distantia. 239. Glechoma (Labiatae $f_{\text {. }}$ ) Antherarum singulum par in formam crucis conuivens. 240. Lamiun (Labiatae f.) Corollae labium super. fornicatum, infer. bilobum, faux inflata utrinque margine dentata.

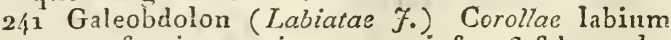
super. fornicatun integrunı, infer. 3-fidum, laciniis acutis.

2.42. Galeopsis (Labiatae $\mathcal{f}$ ) Corollae labium super. fornicatum subcrenatum, infer. ad fancem bifornicato-dentatum. 
243. Betonica (Labiatae f.) Cal. aristatus. Corollae labium super. adscendens planiusculum, infer. trifidum.

2.44. Stachys (Laỏiatae 7.) Corollae labium super. fornicatum, infer. 3-fidum. Stam. minora dellorata ad latera curvata.

245. Ballota (Labiatae 7.) Cal. hypocrateriform. 10-striatus. Corollae labium superius crenatum concavum.

246. Marrubium (Labiatae f.) Cal. hypocrateriform. rigidus 10.dentat. 10-striatns. Corollae la. bium super. rectum lineare bifidum.

247: Leonurus (Labiatae f.) Corollas labium sujerius planum integrum, infer. 3-lobum, labio medio indiviso.

2 f9. Origanum (Labiatae f.) Corollae labium super. planum, infer. 3-partitum, laciniis subaequalibus.

25.3. Verbena (Verbenaceae 7.) Cor infundibuliform. subaequalis curva.

$$
\text { * Cal. 2-labiati. Achaen. 4. }
$$

248. Clinopolinm (Labiatae F.) Involucr, multisetum verticillo subjectum. Corollae labium super. planum emarginatum.

250. Thymns (Labiatae f.) Calycis faux villis clatio

sa. Corollae labium super. planum emarginatum. 251. Scutellaria (Labiatae f.) Cal. truncatus, labio: super. calcarato dein calycem claudente. Corollae

faux compressa.

252. Prunella (Labiatae f.) Filam. 2-furca, inferiore apice antherifera, Stigm, 2 -fidum. 


\section{ATOMOGYNIA.}

- Cal. 4-fidi v. 4-dentati. Caps.

257. Lathraea (Orobancheae.) Cor. 2-labiata. Nect. glandula ad basin germinis.

254. Rhinanthus (Scrophularinae R. Brown.) Cal. inflatus. Corollae labium super. compressum. 255. Euphrasia (Scrophularinae R.Br.) Cal. cylindricus. Anth. spinosae.

256. Melampyrum (Scrophularinae $R$ Br.) Corollae labium super. compressum margine replicatum. * Cal. plerumque 5-fidi v. dentati. Caps. 258. Pedicularis (Scrophularinae R. Br.) Cal. 2-5fidus. Cor. ringens, labio super, galeaeformi. 259. Linaria (Scrophularinae R. Br.) Cor. personata calcarata.

260. Antirrhinum (Scrophularinae R. Br.) Cor. personata ecalcarata.

261. Scrophulatia (Scrophularinae R. Br.) Cor. resupinata 2-labiata, tubo globoso-ventricoso. 262. Digitalis (Scrophularinae R. Br.) Cor. campanulata ventricosa. 263. Limosella (Scrophularinae R, Br.) Cor. campanulata subaequalis. 264. Linnaea (Caprifoliaceae 7.) Involucr. 4-pliyllum. Cor. campanulata. 


\section{TETRADYNAMIA.}

\section{SILICUIOSA.}

- Silicula non emarginata.

265. Berteroa (Cruciferae f.) Petala 2-loba. Stam. minora dentrta. Silicula elliptica $\nabla$. obovata, val$\nabla$ ulis planiusculis.

267. Erophila (Cruciferae 7.) Petala 2-partita. Silicula ovalis v. oblonga, valv. planiusculis.

272. Camelina (Cruciferae 7.) Silicula obovata globosave, valv. ventricosis, loculis $2-$ polyspermis.

273. Neslia (Cruciferae f.) Silicula subglobosa, valv. concavis, septo evanido 1-locularis 1-sperma.

* Silicula emarginata.

266. Alyssum (Cruciferae f) Stam. dentata. Silicula orbicularis v. elliptica stylo apiculata, valv. planis v. centro convexis.

268. Cochlearia (Cruciferae 7.) Cal, patens, Silicula ovato-globosa v. oblonga, valv. ventricos. crassiusculis.

269. Thlaspi (Cruciferae 7.) Silicula depressa, valv. navicularibus dorso alatis.

270. Capsella (Cruciferae 7.) Silicula triangularis depressa, valvul. carinatis non alatis.

271. Teesdalia (Cruciferae f.) Petala inaequalia. Stam. squamula aucta. Silicula ovalis depressa, valvul. navicularibus.

274. Senebiera (C'ruciferae 7.) Cal. patens. Silicula didyma aptera compressa, valinu. subglo. bosis $v$, subcarinatis. 
275. Lepidium (Cruciferae 7.) Silicula ovalis v. subcordata, valv. carinatis vo rarius ventricosis.

\section{SIIIQUUOSA.}

276. Nasturtium (Cruciferae 7.) Cal. patens. Siliqua teretiuscula, valvul, concav. enerv. ecarina. tis. Sem. 2-serialia.

277. Barbarea. (Cruciferae f.) Cat. erectus. Glandulae inter flamenta breviora et pistillum. Siliqua 4-gono-anceps, valv. apice non cornigeris. Scm. 1-serialia.

278. Turritis (Cruciferae f.) Cal. laxus, Siliqua elongata anceps. Sem. 2-serialia. 270. Arabis (Cruciferae f.) Cal. erecins. Siliqua linearis, stigmate subsessili, valv. planis 1-nerviis. Sem. i-serialia.

280, Cardamine (Cruciferce f.) Cal. subclausus, Siliqua linearis compressa, valv. plan. enerviis, saepius elastice desilientibus. Sem. I-serialia. 2\$1. Sisymbrium (Cruciferae f.) Cal. clausus parensve. Siliqua teretiuscula, valv. concavis. Sem. 1.serialia.

282. Alliaria (Cruciferae 7.) Cat. Iaxns. Siliqua te. retiuscula, lineis prominul. subtetragona. 283. Erysimum (Cruciferae f.) Cal, clausus. Siliqua valv. carinatis 4 -gona. 284. Brassica (Cruciferae f.) Cal. erectus subpatens. Siliqua teretinscula, stylo parvo obtuso terminata, $S \in m$, l-serialia.

285. Sinapis (Cruciferae f.) Cal. patens. Siliqua te: retiuscula, valvis nervigeris, stylo parvo breyi acuto apiculata. Sem. -serialia. 
2\$6. Raphanus (Cruciferae 7.) Cal. erectus. Siliqua uransverse in articulos pluimos secedens.

XV. MONADELPIIA.

1. PENTANDRIA.

257. Erodium (Geranioideae 7.) Cor.5-petala. Stam, 5 sterilia c. fertilibus alternantia.

2. DECANDFIA.

28S. Geranium (Geranioideae 7.) Cor. 5-petala. Nect. glandulae 5 ad basin staminum longiorum.

3. POLYANDRIA.

299. Althaen (Malsaceae $\mathfrak{f}_{\text {. }}$ Cal. duplex exterior 1.sejalus $6-12$-fidus, interior 5-fidus. Cor. 5petala.

2go. NIalva (Malvaceae f.) Cal. duplex exterior 3-sepalus, interior 1-sepalus -5-fidus. Cor.5-petala, Linum. Oxalis. Plures Leguminosae.

\section{XVI, DIADELPIIA.}

1. HEXANDPYA.

291. Corydalis (Fumariaccae f.) Cal. deciduus v. ๑. Cor. 4-petala papilionaceo-ringens calcarata. Caps. siliquiformis.

292. Fumaria (Fumariaccae f.) Cal. deciduus. Cor. papilionaceo-ringens calcarata, Drupa siliculiEormis.

2. OCTANDPIA.

293. Polygala (Polygaleae Link.) Calycis sepala 2 majora alaeformia, Cor, 1-petala tubo fissa. Caps. 


\section{DECANDRIA.}

- Stam. monadelpha. Cor. papilionacea. 294. Spartiun (Leguminosae 7.) Cal. 2-labiatus deorsum productus. Legumen compressum. 295. Genista (Leguminosae 7.) Cal. 2-labiatus, labio infer. 3-dentato, super. bidentato. Vexillum re. flexum. Legum. varium. 296. Ononis (Leguminosae 7.) Cal. 5-fidus. Vexillum striatum. Legum. turgidum. 298. Anthyllis (Leguminosae f.) Cal. 5-dentatus ventricosus.

- Stam. diadelpha. Cor. papilionacea. + Stigma pubescens. 299. Phaseolus (Leguminusae f.) Cal. 2-labiatus. Carina cum genitalibus in spiram contorta. 300. Pisum (Leguminosae 7.) Cal. 5-dentatus. Vexillum amplum. Styl. 3-angular. supra carinatus. 301. Orobus (Leguminosae 7.) Cal. 5-dentatus. Vexillum obcordatum. Styl. filiformis. 302. Lathyrus (Legum. 7.) Cal. 5-dentatus. Vexile lum amplum. Styl. planus superne latior. 303: Vicia (Legum. F.) Cal. 5-dentatus. Styl. linearis sub stigmate utrinque villosus. 304. Ervum (Leguminosae 7.) Cal. 5-fidus. Styl. inHexus apice pubescens. Legum. breve.

t+ Stigma glabrum. Legumina non arti= culatim secedentia.

305. Cicer (Leguminosae f.) Cal. 5ofidus corollae longitudine. Styl. filiformis superue pilosus. Le. gumen breve. 297. Robinia (Leguminosae f.) Cal. campanulatus 
4. fidus, lacinia super, saepe 2-fida. Legum. gibbum elongatum.

309. Galega (Leguminosae 7.) Cal. 5-dentatus, dentibus subulatis. Legum. lineis obliquis notatum. 313. Lotus (Leguminosae 7.) Cal. tubulosus 5-fi. dus. Carina superne clausa. Legum. cylindricum rectum.

314. Medicago (Leguminosae L.) Cal. 5.fidus subcampanulatus. Carina snperne clausa demum fissa. Legum. curvatum, falcatum v. cochleatum. 312. Trifolium (Leguminosae L.) Cal. 5-dentatus persistens. Cor. persistens. Legum. indehiscens. 311. Melilotus (Leguminosae L.) Cal. 5-dentatus persistens. Cor. decidiı. Legum. superne dehiscens.

310. Astragalus (Leguminosae L.) Cal. 5-dentatus. Legum. marginibus valvularum intus flexis ma. gis minusve 2-loculare.

++ i Stigma glabrum. Legumina articulatim secedentia.

30S. Hedysarum (Leguminosae L.) Cal.5-dentatus, dentibus subulatis. Carina obtusissima. Legum. subcomipressum.

306. Cosonilla (Leguminosac 7) Cal. 2-labiatus, labio superiore 2-, inferiore 3-dentato. Vexillum alis longius. Legum. rectum, loculis cylindraceis.

307. Ornithopus (Leguminosae 7.) Cal. 5-dentatus tubulosus. Legum. teres arcuatum. 


\section{XVII, FOLYADELPIIA.}

1. POLYANDRIA.

315. Hypericum (Hypericoideae f.) Cal. 5-partitus, Cor. 5-petala. Caps.

\section{SYNGENESIA。}

1. AEPUAIIS.

a) Cor. omnibus ligulatis.

+ Pappus pilosus. Receptac. plerumque nu. rlunz.

316. Tragopogon (Compositae $L$ ) Involucr. aequale pyramidatum sub-10-fidum. Papp. stipitatus. 317. Scorzonera (Compositae L.) Involucr. polyphyllum obiongum imbricatum foliolis margine scariosis. Papp. sessilis.

318. Sonchus (Compositae L.) Involucr. imbricatum basi ventricosum. Papp. sessilis. 319. Lactuca (Compositae L.) Involucr. imbricatum oblongum, foliol. margine scariosis. Pupp. stipitatus.

320. Chondrilla (Compositae L.) Involucr. duplex,

interius cylindricum sub.10.phyllum. Papp. sti.

pitatus.

321. Prenanthes (Compositaz L.) Involucr. duplex, interius sub-5-phyllum. Papp, subsessilis. 322. Leontodon (Compositae L.) Involucr. duplex, exterius foliola breviora, inferius erecta. Papp. stipitatus. 323. Apargia (Compositae L.) Lupolucr, imbrica. 
tum, Receptac. villosiusculum, Papp. sessilis plu. mosus, radiis inaequalibus.

325. Picris (Compositae L.) Involucr. duplex exterius brevius, interius erectum aequale polyphyl-

lum. Papp. sessilis plumosus.

326 Ifieracium (Compositae L.) Involucr. ovatum

imbricatum. Receptac. subsetosum. Papp. sessilis. 327: Crepis (Compositae L.) Involucr. duplex, exterius breve patulum deciduum, interius basi ven. tricosum. Receptac. pilosiusculum.

+ + Papp. radii membranaceus, disci plumosus. Receptac. non paleaceum.

324. Thrincia (Compositae L.) Inyolucr. 8-angulare s.phyllum.

$+\div+$ Papp. pilosus. Receptac. paleaceum. 328. Hypochoeris (Compositae L.) Involucr. imbri: catum. Achaen. difformia. Pupp, radii et disci difformis.

329. Achyrophorus (Compositae L.) Involucr. obIongum imbricatum. Achaen, uniformia. Papp. otipitatus uniformis.

$+\div+\dagger$ Papp. o. v. membranaceus. Rece. ptac. non paleaceum.

330. Lapsana (Compositae L.) Involucr. duplex, exterius brevissimum. Papp. o.

331. Arnoseris (Compositae L.) Involucr. simplex polyphyllum. Papp. margo coriaceus erectus integerrimus.

332. Cichorium (Compositae L.) Involucr. duplex, exterius breve. Receptac. nudum v. setosum. Papp. squamae 5 v. plures, 
b) Cor. omnibus tubulosis, receptac. paleaceo v. alveolaio.

33.3. Arctium (Compesitae L.) Involucr. globosum imbricatum, foliol. (squamis) apice hamis reHexis. Receptac. paleaceum. Papp. brevis.

334. Serratula (Compositae L.) Involucr. cylindri-

cum imbicatum inerme, Receptac, paleaceum. Papp. inaequalis pilosus.

335. Carduus (Compositae L.) Involucr. ventricosum inbricatum, foliol. simpliciter spinosis. Receptac paleaceo-setosum. Papp. pilosus, radiis basi in anmulum connatis, simplicib. v. scabris v. plumosis.

336. Onopordon (Compositae L.) Involucr.ventricosum imbricatum, foliolis simpliciter spinosis. Receptac. alveolatum. Papp. pilosus, radiis in annulum connatis.

337. Carlina (Compositae L.) Involucr. ventricosum duplex, exterioris foliolis pinnato-spinosis, interior is scariosis. Receptac paleaceum. Papp. pilosus.

c) Cor. omnibus tubulosis v. marginis liguiatis. Papp. aristae.

338. Bidens (Compositae L.) Involucr. duplex. Receptac. convexum.

d) Cor. omnibus tubulosis, receptac. nudo. 339. Eupatorium (Compositae L.) Involucr. oblongum imbricatum. Styli profunde 2 -fidi. 340. Chrysocoma (Compositae L.) Involucr. hemisphaericum v. ovatum imbricatum. Styli corollis vix longiores. 


\section{SUPERFLUA.}

\section{a) Cor. omnibus tubulosis.}

3f1. Tanacetum (Compositae L.) Involucr. hemisphaericum imbricatum, foliol adpressis. Receptac. nudum. Papp. margo brevis.

342. Artemisia (Cumpositae L.) Involucr, ovatum

v. subglobosum imbricatum, foliol. adpressis.

Receptac. nudum v. villosum. Papp. o.

343. Guaphalium (Compositae L.) Iruvolucr. imbricatum, foliol. inaequalib. scarios, saepe colora. tis. Receptac nudum. Papl'. pilosus scaber v. pilosus.

b) Cor. marginis radiantibus s. ligulatis. * receptac. nudum, pappus pilosus sessilis. 344. Erigeron (Compositae L.) Involucr. cylindricum $\nabla$. hemisphaericum imbricatum, foliol. angustis apice patulis.

345. Tussilago (Compositae L.). Involucr polyphylInm, foliol. aequalib. simplici serie dispositis. 3.46. Senecio (Compositae L.) Involucr. cylindricum duplex, exterius brevius, foliol. apice sphacelatis. 347. Aster (Compositae L.) Involucr. inbricatum subsquarrosum, foliol. infin. patentibus. (Flor. disci radiantes plures quam 10 lilacini) 348. Solidago (Compositue L.) Involucr imbricatum, foliol. infim. conniventibus. (Flor. disci radiantes 5 v. paulo plures lutei.) 349. Cineraria (Compositae L.) Involucr. polyphyllum simplex, folrol. aequalibus. 350. Inula (Composirae L.) Involucr. imbricatum polyphyll. subsquarrosum, foliol. inferiorib. patulis. Antherae in setas 2 desinentes. 
† + receptac. nudum, papp. plerumque o, vel margo memuranaceus.

351. Bellis (Compositae L.) Involucr. hemisphaericum polyphyllum, foliol. duplici serie disposit. aequalibus. Receptac. conicun.

352. Chrysanthemum (Compositae L.) Involucr. hemisphaericum inbricatum, foliol. margine scariosis. Receptac. convexum. 353. Pyrethrum (Compositae L.) Involucr. hemisphaericum imbricatum, foliol. margine scariosis. Receptac convexum. Papp. margo. 354. Matricaria (Compositae L.) Involucr, imbricatum, foliol. herbaceis. Receptac. conicum. $+\div+$ receptaculum paleaceum.

355. Anthem is (Compositae L) Involucr. hemisphaericum imbricatum, foliol. plus minusve scariosis. Receptac. convexum. Papp. o. v. margo membranacens.

3j6. Aclillea (Compositae L.) Involucr. ovatum inbricatum, foliol. adpressis. Receptac. planum. Papp. 0.

357. Galingsogea (Compositae L.) Involucr. hemisphaer. imbricatum. Papp. e squainis plurib, paleacis compositus.

358. Centaurea (Compositae L.) Involucr. imbricatum, foliol. apice laceris ciliat. v. spinosis. Receptac, paleaceo-setosum.

4. NECESSARIA.

359. Calendula (Compositae L.) Involucr. simplex polyphylium. Receptac, planum. Papp. o, Achaen. difformia. 


\section{GYNANDRIA.}

1. MONANDRIA.

a) Anthera adnata subterminalis persistens. 360. Orchis (Orchideae f.) Cor. ringens, petalo sıperiore fornicato. Labellum basi subtus calcara. tum.

361. Ophrys (Orchideae f.) Cor. subringens, petal. patentibus. Labellum ecalcaratum patens. b) Anthera stigmati parallela persistens. 362. Goodyera (Orchideae f.) Cor. ringens, petal. exteriorib, anticis labello inferne gibboso, superne indiviso suppositis. Columna libera. 363. Neotlia (O-chideae f.) Cor.ringens, petal. ex. teriorib. anticis labello imberbi suppositis, inte. riorib. comniventibus. Columna aptera. 96\%. Listera (Orchideae f.) Cor. irregularis, Labcllum 2-lobum. Columna aptera. 36.5. Neottidinm (Orchideae f.) Cor irregularis. Labellum bilobnm. Columna postice appendice nobili antheriformi.

c) Anthera terminalis persistens. 366. Epipactis (Orchideae f.) Labellum inferne ventricosum superne $v$. indivisum v. 3-lobum, lobo medio articulatim connexo.

d) Anthera terminalis mobilis decidua. 367. Malaxis (Orchideae f.) Corollac petala 5 angustiora. Labell. explanatum indivisum sessile. 2. HEXANDRIA. 368. Aristolochia (Asarinae Kth.) Cor, 1-petala ligulata. 


\section{MIONOECIA.}

\section{MIONANDRIA.}

369. Zannichellia (Potamophilae Rich.) Flor. $0^{7}$ et 오 in eadem spatha. Fior. o. Cal. o. Cor. o. Flor. Cal. campanulatus. Pistilla 3-5. Achaen. 3-5.

2. DIANDRIA.

370. Typha (Typhaceae 7.) Flor. spicati. $\sigma^{\pi}$ supra ㅇ. Flor. $0^{x}$. Cal. obsoletus 3-phyllus simplex. Flor. ‥ Cal. et Cor. o. Achaen 371. Sparganium (Typhaceae f.) Flor. glomerali. ò supra 옹. Flor. ㅇ․ Cal. simplex 3-phyllus. Flor. ㅇ Cal. 3.phyllus. Achaen. 372. Carex (Cyperoideae f.) Flor. spicati varie dis. positi. Flor. $\sigma^{7}$. Cal. gluma. Flor. ㅇ. Cal. gluma. Cor. 1-petala ventricosa. Achaen.

\section{TETRANDRIA.}

373. Littorella (Plantagineae f.) Flor. radicales. Flor. ō pedunculati, Cal. 4-partitus. Cor. 4-fida. Stam. Iongissima. Flor. O sessiles. Cal. squamae. Cor. tubulosa. Achaen.

374. Alnus (Betutincie Rich.) Flor. ơ. Amentum cylindricum, squamis cuneatis 3-foris. Involucr. squama. Cal. 4-partitus. Cor. o. Flor. O. Amenta ovata, squamis 2-floris. Cal. o. Cor. o. Nux inalata.

375. Norus (Urticeae 7.) Flor. ठ․ Amentum. Cal. 4-partitus. Cor. o. Flor. ㅇ. Amentum. Cal. 4-5sepalus. Cor. o. Styl. 2. Achaen. calyce baccato tectum.

3-6. Urtica (Urticeae f.) Flor. ơ. Cal. 4-sepalus. 
Cor. o. Nect. centrale. Flor. 오. Cal. 2-4-sepalus. Cor. o. Caryopsis calyce tecta.

\section{PENTANDRIA.}

377. Xanthium (Ambrosiaceae?) Flor. o'. Involucr. imbricatum. Receptac. paleaceum. Cor. infundibuliform. 5-fidae. Anth. 5. Flor. 오 Involucr. 2phyll. 2.florum, Cor, o. Achaen. 2.

378. Amaranthus (Amaranthaceae 7.) Flor. $\sigma^{7}$ et . Cal. simplex 3-5-phyllus (sepalus). Caps. circumiscissa.

5. POLYANDRIA.

379. Ceratophyllum (Najades). Flor. ơ. Cal. simplex multipartitus. Antherae sub-4-loculares. Flor. ㅇ. Cal. maris Pistyllum 1. Achaen. 1. 380. Myriophyllum (Hygrobiae Rich.) Flor. $\sigma^{\top}$ et Cal. 4-sepalus. Cor. 2-4-petala, petal. caducis. Stam. S. Stigm. 2-4 sessilia. Achaen. 2-4. 381. Sagittaria (Alismaceae R. Br.) Flor. $\sigma^{\circ}$ et 오. Cal. 3-sepalns, Cor. 3-petala. Achaenia. 382. Poteri:m (Rosaccae 7.) Flor. o'. Cal. 4-sepalus, sepalis receptaculo insertis. Cor. o. Flor. 오. Cal. et Cor. maris. Achaen. 2. 383. Betula (Betulinae Rich.) Flor. ơ. Amentum squain. 1-floris. Cal. squama 3-5-partita. Cor.o. Slam. 6-12. Flor. 오. Amentum squamis 2-3-floris. Cal. o. Cor. o. Styl.2. Samara. 384. Quercus (Cupuliferae Rich.) Flor. О`. Amenta nuda. Cal. 5-9-fidus. Cor. o. Stam. 5-1o. Flor. 오. Cal. 1-sepalus, exins imbricato-squamosus. Cor. o. Stigm. 2-5. Nux coriacea calyce cario. ceo-lignesccnte cincta. F. B. 
385. Corylus (Cupuliferae Rich.) Flor. ơ: Amentum squamis 1-floris. Cal. squama 2-partita Cor. o. Stam. 8. Flor. ․ Cal. 2-partitus lacerus. Cor. o. Styl. 2 Nux calyce cincta.

386. Fagus (Cupuliferae Rich.) Flor. o'. Amentum nudum. Cal. 1-sepalus 5-fidus. Stam. $8-12$. Flor. O. Involucr. setaceum 2-forum. Cal. super. 6-dentatus. Nuces 2 involucro aculeato cinctae. 387. Carpinus (Cupuliferae Rich.) Flor. o'. Amentum, squamis 1.floris. Cal. o. Cor. 0. Stam. 1020. Flor. ‥ Amentum gracile, squam. 2-floris. Involucr. squama ciliata. Cal. 4-6-dentatus persistens. Styl. 2. Nux compressa.

358. Platanus (Platanoideae.) Flor. ơ. Amentum globosin. Cal. o. Cor. o. Antherce duplicatae. Flor. ㅇ. Amenta globosa. Cal. et Cor, maris, Styl. 1. Achaen. stylo mucronatum.

6. NONADELPHIA.

389. Pinus (Coniferae 7 .) Flor. $\sigma^{\top}$. Cal. squamosus. Cor. o. Stam. plurima racemosa. Antherae duplicatae c. squama connatae. Flor. ㅇ. Amentum, squamis 2-Aloris. Involucr. squama. Cal.o. Cor.o. Styl. 1. Nu.x.

390. Thuja (Coniferae f.) Flor. o'. Amentım, squam. 1-floris. Cal. o. Cor.o. Antherae plerumque 4 subsessiles. Flor. ‥ Amentum, squam 2-loris. Cal.o. Cor. o. Styl. 1. Nux

391. Bryonia (Cucurbitaceae f.) Flor. O7. Cal. 5dentatus. Cor. 5-partita. Filam. 3. Flor. ․ Cul. et Cor. maris. Styl. 3.fidus. Bacca. 


\section{DIOECIA.}

1. MONA NDRIA,

392. Najas (Najades) Flor. o’. Cal. o. Cor. o. Flor. ㅇ. Orarium stylo 2-5-fido. Achaen. drupaceum. 2. DIANDRIA.

393. Salix (Saliceae Rich.) Flor. ठ․ Amentum squam. ciliat. 1-floris. Nect, glandula staminifera. Flor. ㅇ. Amentum maris. Nect, glandula germinifera. Styl, 2-fidus, Caps.

TRIANDRIA Valeriana dioica.

3. TETRANDRIA.

394. Viscum (Viscoideae Rich.) Flor. ơ. Cal. 4-partitus. Anther. calyci insertae. Flor. ㅇ. Cal.4.fi. dus. Bacca.

Urtica dioica.

4. PentandRiA.

39\%. Spinacia (Chenopodeae DC.) Flor. $0^{7}$. Cal. 5paritus. Flor. ‥ Cal. 2-4-fidus. Cor. 0. Styl. 4. Achaen.

396. Cannabis (Urticeae f.) Flor. ơ. Cal. 5-parti. tus. Cor. o. Flor. ‥ Cal. 1-sepalus, lateribus his ans. Cor. o. Styl. 2. Achaen.

397. Humulus (Uricicae $\mathcal{F}_{\mathrm{i}}$ ) Flor: $\sigma^{7}$ Cal. 5-sepa. lus. Flor. ‥ Cal. 1-sepalus oblique patens. Cor. patens subglobosa 1-petala germen arcte cingens. Styl. 2. Achaen. $R$ hamnus catharticus. HrXANDria Asparagus officinalis. Rumex Acetosa, Acetosella. 
5. OCTANDRIA.

398. Populus (Saliceae Rich.) Flor. O`. Amentum, squam. laceris I-floris. Cal. turbinato-cyathiformis. Flor. O. Amentum et Cal, maris, Stigm, sub4-partitum, Caps.

6. ENTEANDRIA.

399. Mercurialis (EuphorBiaceae f.) Flor. o. Cal. 3-partitus. Stam. 9-10. Flor. 오. Cal. maris, Styl. 2. Caps.

400. Hydrocharis (Hydrocharideae R. Br.) Flor. $\sigma^{7}$.

Spatha 2-phylla. Cal. 3-fidus. Cor. 3-petala. Filam. plurima. Styli 3 imperfecti in medio. Flor. \&. Cal. et Cor. maris. Caps.

Decandra Cucubalus Otites, Lychnis dioica.

7. DODECANDRIA.

401. Stratiotes (Hydrocharideae R. Br.) Flor. $\sigma^{7}$. Spatha 2-phylla. Cal. 3-partitus. Cor. 3-petala. Stam. in $\Varangle 20$ fertilia in $\sigma^{\top} .9-12$ antherifera, reliqua antheris destituta. Flor. ‥ Spatha, cal., cor. maris. Styl, 6-partiti. Bacca.

8. MONADELPHIA.

402. Juniperus (Coniferae f.) Flor. ठ. Amentum. Cal. o. Cor. o. Stam. in flore terminali $3 \mathrm{cum}$ filament. brevibus, in squamuli flore c. $2-6$ sine filamentis. Flor. Q. Amentum, cal., cor. maris. Germ. 1. Styli 3. Bacca c. amenti squamis coalita. 103. Taxus (Coniferae 7.) Flor. ơ. Amentum. Cal.o. (nisi gemma squamae) Cor. o. Stam. plurima, antheris peltatis. Flor. O. Cal. et cor. maris. Germ.1. Styl. o. Nux receptaculo baccato cincta. Bryonia dioica. 


\section{CRYPTOGAMIA.}

\section{IIIICES.}

a) Fructus in racemum strobuliformem dispositi. Receptac. pettata. Caulis aphyllas verticillatum ramosus vaginatus.

404. Equisetum (Equisetinae R. Br.)

b) Fructus in spican dispositi $v$ axillares. Caps. 1-loculares 2-valves. Caulis plerum. que foliosus.

405. Lycopodium (Lycopodineae $S_{w a r t z)}$ Caps. reniformes.

406. Ophioglossum (Ophioglossinae R. Brown.) Caps. transverse dehiscentes in spicam articulatam connatae.

407. Botrychium (Ophioglossinae R. Br.) Caps. transverse dehiscentes discretae rhachidi racemi compositi adnatae.

c) Fructus in paniculam dispositi frondem con tractam tegentes. Caps. semibivalves.

40S. Osmunda (Osmundaceae R. Br.)

d) Fructus in frondis dorso yel margine dispositi. Caps. annulo articulato instructae (gyratae) I-loculares irregulariter dehiscentes.

40g. Polypodium (Polypodiaceae R, Br.) Soridorsales subrotundi sparsi. Indusium o. 410. Aspidium (Polypodiaceae R. Br.) Sori dorsales subrotundi sparsi. Indusia.

411. Asplenium (Polyporliaceae R. Br.) Sori dorsa. les lineares transversales sparsi. Indusia. 
413. Blechnum (Polypodiaceae R. Br.) Sori dorsales lineales longitudiuales continui costae frondis utrinque paralleli. Indrusia.

412. Pteris (Polypodiaceae R. Br.) Sori lineares marginales continui. Indusia.

d) Fructus radicales. 415 Pilularia (Marsiliaceae $R . B r$.) Caps. 4-6-locularis.

416. Salvinia (Marsiliaceae R. Br.) Caps. 1-locularis.

A T.GAE.

417. Chara (Charae?) Globuli epigyni. Nucula subturbinata. (Caulis verticillatim ramosus.) 


\section{1. I. MON A N D R I. ORDO I. MONOGYNIA.}

1. HIP U IRI

Cal. integer minimus superus. Cor. o, Siyl. anthe. rae sulco receptus. Nux $\mathbf{1}$-sperma $\mathbf{1}$-locularis. 1. H. vulgaris: fol. verticillatis plurimis $(8-12)$ linearibus acutis. Vahl. Enum. I. 13.

๙. palustris. \&. fluviatilis. $\%$ ramiger. S.I. 1. A. $\frac{1}{2}-2^{\prime} 2 \%$ Fl. 6, 7 . In ripis lacumm et llumiuum acervatim. Thierg. Frankf. Th. Span. dau, Charlottenburg etc.

\section{ORDO II. DIGYNIA.}

2. CALLITRICHE.

Cal. o. Cor. 2-petala infera. Samarae 2-4 appro. ximatae.

1. C. verna: fol. obovatis linearibusque, summis 3-plinerviis, samaris obtuse marginatis. S I. 2.

Callitriche autumnalis. Kth. 2.

a. platypliylla. B. hethopisylla. $\boldsymbol{\gamma}$. isophylla. 8. leptophylla. Fi. $4-8$. (- (?) 24 (?). Flor. polygami. Cor. alba. Planta variabilis fere undique in aquis $v$. solo humido acervatim copiosa. F $B$ 


\section{C1. II. DIANDRIA. \\ ORDO I. MONOGYNIA.}

3. I I G USTRUM.

Cal. 4-dentatus. Cor. infundibilifornis 4-fida. Bacca 2-locularis, loculis 2-spermis.

3. L. vulgare: fol, ovali-lanceolatis, thyrsis paniculatis terminalibus. H. A. V. 25.

a. germanicum. B. italicum. A. 6-8' 万. Fl. 6, 7. Cor. alba, Bacca nigra. In sylvis et sepibus passim e. c. Thierg. Panckow etc.

4. SYRINCA.

Cal. 4-dentatus. Cor. hypocrateriformis margine 4-fido. Caps, 2-locularis 2-valvis. Sem, margine membranaceo cincta.

1. S. vulgaris: fol, ovato-cordatis. L. W.I. $4 \mathrm{~S}$. A. 15 - $20^{\circ}$ 万. Fl. 5. Cor. violacea et alba. In sepibus et nemoribus hinc inde obvia. Thierg. Panck. Schönh, etc.

5. VERONICA.

Cal. 4-5-partitus. Cor. rotata limbo 4-partito, lacinia infima angustiore. Caps. 2-locularis polysperma, valv. medio septiferis.

* Racemis terminalibus spicisve, fol. verticillatis et oppositis.

1. V. longifolia: fol. oppositis 3-4-ve cordato- 
Ianceolatis acuminatis duplicato-serratis caulequo pubescenti-tomentosis. Schr. g. I. 18.

V. maritima. IV. A. 1-3' 2. Fl. 7, 8. Cor. coerulea, Rad. crassa. Inter frutices prope fuminum ripas. Chal lottb. Teg. Strahl. Trept. eic.

2. V. spicata: fol. oppositis crenatis cauleque pubescentibus, radicalibus et canlinis inferioribus oblongo-ovatis in petiolum decnrrentibus, cauli. nis oblongis basi attenuatis. Sclir. g. I. 15.

". monostachya. B. polystachia. S.J.5. Schr.1.1. A. $\frac{x}{2}-24$. Fl. 5,6 Cor. coerulea. In collibus siccis. Jungf h. Papenb. Grunw. Kalkb. etc.

* Racemis terminalibus.

3. V. serpyllifolia: fol. ovatis subcrenatis glabris, bracteis inferioribus foliiformibus, superioribus lanceolatis integerrimis pedunculis fructiferis longioribus, cor. calycem superante. S. I. 6.

A. $\frac{\pi}{4}-\frac{T}{2}{ }^{\prime}$ 2. Rad. subrepens. Fl. 4, 5. Cor. coerulescens rel incarnata. In locis humidis frequens. Thierg. Grunw. Teg. etc. *** Racemis lateralibus.

4. V. Beccabunga: fol. ellipticis petiolatis ob. tusis obsolete serratis glabris, cal. 4-partitis, caule glabro inferne radicante. S. I. 12. H. A. IV. 2. a. major. B. minor. A. $\frac{5}{2}-1 \frac{1}{2}{ }^{\prime}$ 24. Fl. 5, 6. Cor, coerulea. In locis irrignis. Jungfh. Gesundbr. Thierg etc. 
5. $Y$. Anagallis: ful. sessilibus lanceolatis obsolete serratis glabris, cal. 4-partitis, caule plerumque inferue radicante glabro. 'S.I.12. H. A.IV.ı.

«. aquatica. B. terrestris. A. $\frac{1}{4}-2^{\prime} 24 . \mathrm{Fl} .5-7$. Cor. coernlescens. In locis humidis et in aquis. Jungt h. Teg. Trept. etc.

6. V. scutellata: fol. scssilibus lineari-lanccolatis denticulatis et integerrimis glabris, cal. 4-partitis, caule glabro ex procumbenti-erecto. S. 1. 13.

A. 2-8" 4. Fl.6, 7. Cor. coerulescens. In paludibus. Grunw. Jungfh. Charlottb. etc.

7.V. officinalis: fol. obovatis v. obovato-subrotundis serratis, cal. 4-partitis, caule hirto puboscente inferne radicante. S. I. 31. H. A.IV.3.

A. $\frac{1}{4}-\frac{1}{2}$ '4. Fl. 5, 6. Cor. coerulescens. In sylvis frequentissima. Grunw. Teg. etc. Oft.

8. V. prostrata: fol. sessilibus oblongis obtusis serratis, cal. 5-partitis, caule florifero adscendente. Schi. g. I. 38 .

A. $\frac{1}{4}-\frac{1}{2}$ 24. Fl. 4, 5. Cor. coerulea. In collibus arenosis, Jungfh. Charlottenb. etc. frequens.

9. V. latifolia: fol. sessilibus subcardato-ovatis obtusis serratis, cal. 5 partitis, caule adscendente. Schr. g. I. 35 .

A. $1-2,2$. Fl. 6, 7. Cor. coerulea. In solo fertili, Charlottb. Temph. Pank. etc. sparim. 
10. V. Chamaedrys: fol. sessilibus petiolatisve inciso-serratis, summis cordato-ovatis, cal. 4partitis, caule bifariam piloso. S. I. 11.

A. $\frac{1}{2}-1 \cdot 24$. Fl 4, 5. Cor. coerulea. In solo fertiliori ubivis obvia.

* * * Pedunculis unifloris.

11. V. verna: Aor. subsessilibus, fol. digitatopartitis summis indivisis, pedunculis cal. brevio. ribus, caule stricto. Schr. g. I. 45 .

๙. gracilis. B. virgata. $\%$. multicaulis. S. I. 7. A. $2 "-1$ " $\odot$ FI. 4 . Cor. pallide coerulea. In campis arenosis admodum copiosa.

12. V.triphyllos: fol, infimis indivisis, mediis di-

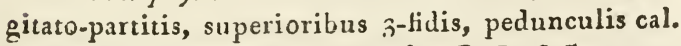
longioribus, caule erecto patulo. L. R. S.I.121.

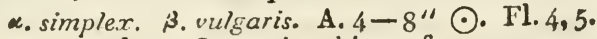

Cor. coerulea. In agris ubique frequens.

13. V. hederaefolia: ful petiolatis, primordialibus oralibus integerrimis, reliquis subcordatorotundis $3-7$-lobis, bracteis his consimilibus pedunculis fructiferis recurvis multo brevioribus, cor. calycem aequante. S. I. 9.

A. $2-6 " \odot$ Fl. 3, 4, 5. Cor. coerulescens. In agris cultis et incultis copiosa.

14. $V$. praecox (Allioni): fol, petiolatis cordato. ovatis errato-crenatis, bracteis sessilibus, inferioribus basi profunde dentatis, sunmis integerrimis, 
pedunculum fructiferum aequantibus, corolla cal. aequante. S. I. $s$.

A. 3-4" (.) Fl. 4. Cor. coerulea. In agris argillosis Spand. Charlottb. W. Gesundbr.

15. V. arvensis: fol. petiolatis cordato-ovatis serrato-crenatis, bracteis sessilibus, inferioribus fol. similibus, superioribus lanceolatis integerri. mis pedunculo fructifero brevissimo multo lon. gioribus, cor. calyce breviore. S. I. 8 .

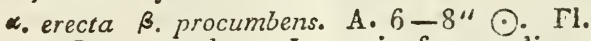
4, 5. Cor. coerulea, In agris fere undique sparsim.

16. $V$. agrestis: fol. petiolatis cordato-ovatis serrato-crenatis, bracteis subsessilibus fol. consimilibus pedunculum fructiferum recurvum aequantibus, cor. calycem aequantc. S.I. g.

A. $6-8$ " $\odot . F 1.4,5$. Cor coertilea. In agris sparsim, sed frequenter, obvia.

6. GRATIOLA.

Cal. 5-partitus bracteis 2-bus. Cor. tubuloso-3labiata, irregularis, resupinata. Stam. 4, 2-sterilia. Stigm.2-labiatum. Caps. 2-locularis $4-v a l v$. G. officinalis: fol. sessilibus lanceolatis 3-5nerviis punctatis a medio serratis, pedunculis axillaribus fol, brevioribus, rad. repente. S. I. 14. H. A. III. 13. 
A $\frac{t}{2}-1,24$. Fl 6, 7. Cor. ex albo rubella. In pritis humidis Charlottb. Strahl, etc. Off. 7. PINGUICUIA.

Cal. 2-labiatus 5-fidus. Cor. ringens calcarata. Caps. 1 -locularis subbivalv. polysperma.

P. vulgaris: calcare subulato cor. laciniarum longitudine, labio superiore 2-lobo, inferioris (3-10bi) laciniis obtusis. Schr. g. I. 52. Mert. g. I. 34I. A. 3-6" 24. Fol. carnosa. Fl. 5, 6. Cor. violacea. In pratis uliginosis. Grunw. Fried. richsf. etc.

\section{UTRICUIARIA.}

Cal. 2-phyllus aequalis. Cor personata $\nabla$. rine gens calcarata. Caps. circumscissa 1-locularis supera.

1. U. vulgaris: calcare conico patente, labio superiore integro palatilongitudine, pedunculis erectis, fol. pinnato-multipartitis laciniis capillaribus aculeato-ciliatis. Mert. g. I. 343 .

A. $6-8$ - Fl. 5, 6, 7. Cor vitellina. Fol. anipullifera. In fossis et pratis turfosis acer. vatim. Grunw. Jungfernh. etc.

2. U. intermedia (Hayne): calcare globoso ad. presso, labio superiore integro palato duplo longiore, pedunculis erectis, fol. 3 partitis laciniis furcatis linearibus angustis ferrulatis. Mert. g I.344. 
1. 6--8"2 4 . Fl. 6. Cor. sulphurea striis purpureis. In fossis turfosis rarius. Grunw. Jung $f$. 3. U. minor: calcare patente carinato, labio su. periore enrarginato palati longitudine, pedunculis declinatis, fol. 3-partitis laciniis capillaribus dichotomis. Mert. g. I. 345.

A. 3-6“24. Fl. 5, 6, 7. Cor pallide lutea. Fol. ampulliferis. In fossis acervatim conspicua. Grunw. Jungfernh. Tassd.

$$
\text { 9. I Y COPUS. }
$$

Cal. tubulosus 5-fidus. Cor. tubulosa subaequalis 4.fida, lacinia superiore latiore emaryinata. Stam. distantia. Achaen 4. depressa.

L. europaeus: fol. petiolatis ovato-lanceolatis grosse serratis subpubescentibus, flor. verticilla. tis. Mert. g. I. 346 .

A. $1-3,2$. Rad repens. F1. 5, 6, 7. Cor. alba punctis rubris. In pratis humidis spar. sim. Grunw. Charlottenb. Strabl.

$$
\text { 10. SAIVIA. }
$$

Cal. subcampanulatus, 2-labiatus, labio superiore 2 -3-dentato, inferiore 2-fido. Cor. ringens. Filam. transverse pedicello affixa. Achaen 4. $S$. pratensis: fol. cordato-oblongis crenatis incisisve, bracteis cal. minoribus, cor. labio superiore viscoso falcato labio inferiore longiore. Schr. g. I. $6_{3}$ H. A. VI. 2 . 
A. 1-2' 24. Rad. lignosa. F1. 5,6. Cor, vio. lacea, rarins alba. In collinm radicibus, campis argillosis, coemeteriis. Teg. Papenb. etc.

1I. CIRCAEA.

Cal. 2.phyllus superus. Cor. 2-petala. Caps. 2locularis, 2-sperma.

C. lutetiana: caule adscendente pubescente, fol. oratis acutis exciso-denticulatis pubescentibus, petalis profunde emarginatis. Mert. g. I. 358.

A. $1-2 \frac{1}{2} \cdot 24$. Fl. 6, 7, 8. Cor. albido-rubens. In sylvis frondosis acervatim. Thierg.

$$
\text { 12. IRAXINUs. }
$$

Flor. polygami. Cal. o. $\nabla$. 4-partitns. Cor. o. $\nabla$. 4-petala. Samara 2-locularis loculis $2-3$-spermis. Sem. plerumque 1. apice alatum, reliquis abortivis.

F. excelsior: fol. impari-pinnatis, foliolis $9-15$ subpeticolatis lanceolatis acuminatis serratis basi cuneiformibus, supra glabris, infra supra costae basin pubescentibus, petivlo semitereti inferne glabro, superne canaliculato pubescenti, Horibus nudis. H. F. 226.

$\alpha$. communis in agris sola obvia varietas. A. $80-100^{\circ}$ t7. Fl. $4-5$. In solo meliori via versus Pank. Thierg, etc.

13. IEMNA.

Cal, 1-phyllus (membranaceus) Cor, o, Caps utri- 
culiformis supera $\mathbf{1}$-locularis $1-2$ ( $\boldsymbol{r}_{0}$ poly-) sperma. (Flor, in rima marginis fol.)

1. L. trisulca: fol. petiolatis elliptico-lanceolatis lateraliter p:oliferis cruciatim connexis 3 -sul. catis, radiculis solitariis. Mert. g. I. 294 .

A. $1-1 \frac{1}{2} "$ ○. Fl. 6, 7. In aquis stagna:1tibus frequens. Thierg. Grumw. etc.

2. L. minor: fol. obovatis ellipricisve utrinque planiusculis basi cohaerentibus, radicnl. solitariis. S. I. 19 .

Antec. similis A. - O. Ioris tempore et locis.

3. L. gibba: fol. obovatis convexiusculis basi cohaerentibus subtus hemisphaericis, rad. solitariis. S. I. 19 .

Antec. similis A. $\odot .$, flor. tempore locisve, sed raxius obvia.

4. L. polyrrhiza: fol. sessilibus rotunato-ob. oratis v. ellipicis convexiusculis, rad. fasciculatis. Mert. g. I. 296.

In praec. locis, quibuscum A. $\odot$. Hor, tem. pore convenit, rarissima.

$$
\text { 14. CL } \triangle \text { D I } \mathrm{M} \text {. }
$$

Cal. multivalvis 1 -florus, valr. glumaceis imbricatis exterioribus minoribus. Cor. o.Achaen. putamine duplici.

C. germanicum: culmo tereti, corymbis confertis, llor. fasciculatis. Schr. g. I. 75. 
A. $2-6 \cdot 24$. Rad. repens. Fol, rigida linea. ria Fl. 6,7 . Acervatim ad ripas lacuum. Schlaclitensee. Talsdorf.

\section{A T THOXA NTHUN.}

Cal. 2-valv. I.llorus, valv. compressis, herbaceo. membranaccis. Cor. duplex, exterior oblonga obtusa, valv. interiore sub apice, superiore basi aristata, interior duplo brevior $2-\nabla a l v i s$ mutica, corollae adnata.

1. A. odoratum: spica ovato-oblonga contracta, culmo glabro, valv. inferiore floris interni 3-ner-vi. Meıt. g.J. 303.

A. $\frac{1}{2}-2,2$ Fol linearia. Fl. 5, 6. In pratis, humidiusculis locis et sylvis frequentissimum.

\section{C1. III. TRIANDRIA. \\ ORDO I. MIO NOGYNIA. 16. VAIERIANA.}

Cal. margo involutus. Cor. 1-petala limbo 5-fido basi hinc inde gibba. Achaen. 1-loculare calycis limbo in pappum plumosum evoluto coronatum.

1. V dioica: caule 4.gono (stolonifero), fol. radicalibus integris, caulinis ly rato-pinnatis, florib. 3-andris dioicis. H. A. III. 31. 
A. $\frac{T}{7}-1: 24$. Fl. 5, 6. Cor. alba $\nabla$. incarnata. In pratis humidis undique frequens. Thierg. Cluarlottenb, etc.

2. V. officinalis: caule tereti sulcato, fol omnibus pimatis, fuliol (lanceolatis) serratis, Horibus 3-andris. H, A. III, 32 .

a. latifolia. 6, angustifolia. A, 1-4' 24. Fl. 7. Cor. carnea v. albida. Fad, praemorsa fusca. $\alpha$. in lnmmidis, $\beta$, in aridis locis. (e. c. Rüdersd,) haud rara, Off.

17. FEDIA.

Cal. margo 3-6-dentatus persistens. Cor. 1-petala inaequaliter 5 -fida ecalcarata basi hinc gibba. Nux calyce persistente coronata I-3locularis 1-sperma.

1. F. oltoria (Steven): fructu glabriusculo compresso orbiculato oblique acuminato utrinque bistriato. S. I. 22.

I'alcriana olitaria $W$.

u. vulgaris. $\beta$. oleracea, A, $2-12^{\prime} \odot-\sigma^{\top}$. Fol. spathulata et ovato-oblonga. Fl. 4,5 . Cor. coerulescens. In campis argillosis undique. Charlottb. Templi, etc.

2. F dentata (Steven): fructu glabro ovato, antice costa unica, postice quinis, apice oblique acuminato. S. I. 23.

Valeriana dentata, W. 
A. $\frac{r}{2}-1^{\circ}$. Fol. spathulata et linearia. Fl. 5, 6. Cor. alba et carnea. In locis antec. Spand. Temph. Charlortb. etc.

3. F. dasycarpa (Steven): fructu pubescente ovato.conico, apice oblique acuminato. S. I. 24.

A. Cor. et locus antecedentis a qua fructibus tantum differt.

4. F. auricula ( $\left.\begin{array}{ll}D & C .\end{array}\right)$ : fructu glabro globosn. ovato, antice costis 2.sulco 1 , postiee costa $1-s u l$ cis 2, apice oblique acuminato. S. I. 2f.

Rarius obvia in locis praecedentium eoden tempore quibuscun habitu externo conve. nit. $S$.

\section{IPIS.}

Cal. o. Cor. 6-partita supera, laciniis alıernis reflexis, alternis conniventibus. $S y l$. petaliformis 3-laciniatus, Stigm. plicatum. Caps. 3-10. cularis 3-valv. polysperma.

1. Peudo-Acorus: fol. eusif mrmibus cauli longitudine subaequalibus paululum curvatis, cor. im. berbibus laciniis exterioribus erectis styl. laciuiis noulto minoribus, gernine trigrono. Mert. g. 1.41\%. A. $2-3 \cdot 2$. Rad gonygoniata. F1. 5, 6. Cor. lutea. In fossis, fluviis, paludibus etc. undique frequens.

2. 1. sibirica: fol. planis linearibus caule tereti fistuloso $2-3$-floro brevioribus, cor. imberbi la- 
ciniis interioribus styl. aequantibus, germine trigono. Mert. g. I. 419 .

A. $2-2 \frac{1}{2} \cdot 24$. Rad. fibrosa. F1. 5-7. Cor. coerulea venis purpureis. In pratis humidis rarius. Rummelsb. WV. Papenb.

19. POLY CNEMUN.

Cal. 5-phyllus inferus bracteis suffultus. Cor. 0. Achaen. membranaceum utriculiforme monospermum.

1. $P$. arvense : caule ramoso divaricato, fol. subulato-prismaticis apice muicatis spinulosis subglabris, flor. axillaribus sessilibus. Mert. g. I. 403. «. erectum. $\beta$. procumbens. S.I. 26. Schr. g. I. 9I. A. $1-6 "$ ○. Fl. 6,7,8. In agris arenosis haud rarum. Iasenli, Grunw. Jungfernh.

$$
\text { 20. CYMERUS. }
$$

Spiculae distichae e glumis 1-valv. 1-flor. com. pressis imbricatis compositae. Glumis omnibus floriferis v. $1-2$ inferioribus sterilibus. Setae lyypogynae o. Achaen. obovatum.

1. C. flavescens: culmo obtuse-triquetro, corymbi ramis simplicibus, spiculis confertis oblongolanceolatis, glumis obtusis, involucr. 3.phyllo, 6tigm. 2. Mert. g. I. 420 . A. $2-8^{\prime}$
$\odot$. Rad. fibrosa. Fol. carinata, li. nearia. Fl. 7,8 . Assiduus incola paludum. Weirsens, Grunw. etc. 
2. C. fuscus: culmo acute-3-quetro, ramis corymbi subsimplicibus, spiculis lincaribus confertis, glumis acutiusculis, involucr. $3 \cdot$ phyllo, stign. 3. Mert. g. I. 421.

A. etc, antec. similis isdem que locis obvius. 21. SCIRPUS.

Spiculae undique imbricatae e glumis $4 \mathrm{v}$. plaribus 1-valv. compositae. Glumae omnes flo. riferae v. $2-3$ vacuae. Setae hypogynae glumis breviores v. o. Achaen. obovatum.

* Spica terminalis simplex.

+ Siyl. basi dilatata ovata persistente, setis hypogynis $4-8 \mathrm{v} .0$. (Eleocharis R. Brown.)

1. S. palustris: culmo subrotundo v. compresso, raginis aphyllis, spicula oblonga, glumis acutiusculis, inferiore breviore spiculam dimidiam cingente, stigm. 2, rad. repente. Mert. g. I. 425. A. $\frac{\mathrm{r}}{2}-2,24 . \mathrm{Fl}, 5, \mathrm{G}$. In locis udis undique fere conspicuus.

2. S. acicularis: culmo 4-angulo sulcato, raginis aply $\mathrm{l}_{\mathrm{y}}$ lis, spiculis ovatis, stigm. 3, achaen. co. stato. Mert. g. I. $42 \mathrm{~S}$.

Eleocharis acicularis R. Brown. Isolepis acicularis. S. 1. 30 .

A. 1-4" () v. 24 ?) Fol. setacea. Fl. 6, 7, 8

In locis paludosis fere undique. 
tot Styl. basi non dilatata, sed mucronata persistente, setis hypogynis. (Scirpus R. Brown.)

3. S. Baeothryon. (Ehrhart.): culmo tereti, vaginis aphyllis, spica ovata, glumis obtusis, inferiore majore inermi spicam involucrante, stigm. 3, achaen. glabro, rad. fibrosa (sobolifera). Mert.g.I. 430. S. I. 32 .

A. $2-8 " 24 . \mathrm{Fl} .6,7$. In pratis acervatim rarius. Kalkb. Jungfermh.

* Spica terminalis v. subterminalis com. posita.

† Seris hypogynis 3-6. (Scirpus. R. Brown)

4. S. caricis (Retz.): culmo subtriquetro folinso, spica disticha, bracteis spicula brevioribus, inferiore in involucr. 1 -phyllum elongato, spiculis 6-8-flor., fol. canaliculato - carinatis margine scabriusculis. Mert. g. I. 446. S. J. 32.

S. caricinus. Schr. g. I. 132. S. compressus Persoon. Mert.1.1.

A. $\frac{1}{9}-1 \cdot 24$. Rad. sobolifera. Fl. $6-8$. In hunidis granimosis acervatim. Spand. Charlottenb. vitc.

++ Setis hypogynis o. (I solep is R. Brown.)

5. S. supinus: culmo tereti, spiculis glomeratis sessilibus involncro monophyllo erecto culmi lon- 
gitudine glumis obtusis breviter mucronatis stigm. 3 , achaen. transversim rugoso. Mert. g. I.439.

Isolepis supina. R. Brown. S. I. 37.

A. 4-10" С. Rad fibrosa. Fol. setacea canaliculata. Fl. $6-\mathrm{s}$. In locis humidis ad margines lacuum rarius. Weilss. S.

6. S. setaceus: culmo tereti, spiculis tcrminalibus subgeminis sessilibus, involucr. culmo multo breviore, glumis obtusis breviter mucronatis, stigm. 3, achaen. longitudinaliter costato compres. so. Mert. g. I. 440.

A. 2-6" $\odot$. Rad. fibrosa subrepens. Fol. setacea. Fl. $6-8$. In locis humidis arenosis, ad lacuum margines. Temph. Grunw. etc.

*** Spiculae in corymbum suprafastigiatum dispositue. Styli basis non dilatata. Setae hypogynae 2-6. (Scirpus R. Brown)

7. S. lacustris: culmo tereti, corymbo composito spiculis fasciculatis, involncr. 2-phyllo, glumis nucronatis emarginatis fimbriatis glabris, stign. 3 , antheris apice barbatis. Mert. g. I. 433 .

a. major. B. minor. Schr. g. I. I34. A. 1$10^{\circ} 24$. Pad, articulata. Fl. 6,7. Fol. semiteretia. In aquarum ripis frequens.

s. S. maritimus: culmo 3 -quetro folioso, spiculis fasciculatis pedicellatis sessilibusque, involucr. F. $B$. 
polyphyllo, glumis apice denticulatis 2 - fidis mucronatis, stigm. 3 . Mert. g. I. 443 .

A. 1-4'24. Fol. 3.quetra linearia. Fl.6,7,8: In locis udis ad fluminum ripas. Spree.

9. S. sylvaticus: culmo 3-quetro folioso, corymbo decomposito, spiculis sessilibus fasciculatis, invo. lucro polyphyllo, glumis obtusis breviter mucrocronatis, setis rectis achaen. longitudine. Mert. g I. 444 .

A. $1-3^{\prime}$ 4. Fol. lanceolato-linearia. Fl.6,70 In solo humido passim. Thiergart. Spand. $\mathrm{Pa}$ penb. etc.

22. SCHOENUS.

Spiculae undique imbricatae $\nabla$. 2.stichae e glumis 6 - 9 tuivalv. c mfositae, glumis inferioribus $3-4$, quarum 2 inferiores angustiores et minores, vacuis, setis hypogynis $3-10$ v. o. Achaen. obovatum v. obconicum setis cinctum $\nabla$. nudum.

* Achaen. (sty $l_{0}$ persistente) rostrato, glumis undique imbricatis, setis hypogynis. (Rhynchospora. Vahl.)

1. S. albus: culno folioso 3-gono, fol. linearibus (carinatis), fasciculis terminalibus subcorymbosis involucr. inferiorem aequantibus, stam. 2, etis 10 , achaen. longitudine, rad. fibrosa. Mert. 58 I. 448 . 
Rhynchospora alba. 'T'ahl. En II. 2,36. S. I. 2s.

A. $\frac{\mathrm{T}}{2}-1 \frac{1}{2} \mathbf{2}^{\prime} 4 . \mathrm{Fl} .7, \mathrm{~S}$. In locis turfosis Tafsd. Grunv. etc.

2. S. fuscus, culmo 3.gono folioso, fol. setaceoangustis, fasciculis terminalibus involucro inferiore multo brevioribus, stam. 3 , setis 3 achaen. duplo longioribus, rad. sobolifera. Mert. g. I. 449 .

Rhynchospora fusca. Vahl. l. l. S. I. 28.

A. $\frac{r}{4}-1 \cdot 24$. Fl. 6, 7. Habitat in pratis turfosis praecedente rarius. S.

* Achaen. obtuso breviter mucronato, glumis distichis, setis hypogynis o. (Schoe. nus. R. Brown.)

3. S. nigricans: culmo tereti (nudo), fasciculo multifloro involucri 2 - phylli valv. exteriore breriore. Mert. g. I. 451 . Schr.g. I. 113. S. I. 29. A. $\frac{T}{2}-1,24$. Rad. caepitosa. Fol. setacea. Fl. 5-7. In pratis turfosis acervatim. Tafsd. 23. ERIOPHORUNI.

Spiculae glumae 1.valv. undique imbricatae inferioribus $1-2$ saepe vacnis. Setae hypogynae $4 \mathrm{~V}$. multae post efflorescentiam longissime (lanae similes) excrescendo cal. superantes ct achaen. obovatum cingentes.

* Spica solitaria terminali.

1. E. vaginatum: culmo superne triquetro glaB 2 
bro. fol. margine scab 1 is, spica orato - oblonga. Mert. g. I. 453. S. 1. 29.

E. caespitosum. Schr. g. I. 150.

A. $\frac{1}{2}-2 \div 24$. Rad. caespitosa. Fol. subulata: Fl. 4,5 . In locis turfosis. Grunw. Jungfh. etc. copiose.

* * Spicis pluribus pedunculatis.

2. E. triquetrum (Hoppe): culmis obtuse-trigo. nis, fol. triquetris, spicis pluribus pedunculatis pedunculis tomentoso-scabriusculis, achaen. oblongis triquetris. Mert. g. I. 456. S. I. 30.

E. gracile. Koch. Mert. 1. I. E. polystachyrm. W. A. 1-1 $\frac{r}{2} \cdot 2$. Rad. sobolifera Fl. 5, 6. In locis antec. sparsim. Jungfh. Grunw. Tafsd.

3. E. angustifolium (Roth): culmo teretiusculo subtrigono, fol. canaliculato-carinatis in apicem longum triquetrum desinentibus, spiculis pedicellatis pluribus, pedunculis glabris, achaen. obovatis. Mert. g. I. 455. S. I. 30.

A. $\frac{r}{2}-2,24$. Rad, sobolifera. Fl. 4, 5. In pratis et paludibus frequentissimum.

4. E. latifolium (Hoppe): culmo obtuse triquetro, fol. planis apice triquetris, spicis pluribus pedunculatis, pedunculis (retrorsum) scabris, achaen. obovatis triquetris. Mert. g. I. 454. S. I.31.

A. $1-2,24 . F 1.4,5$. In paludibus turfo. sis. Grunw. Jungth. etc. 


\section{NARDU3.}

Cal. o. Cor. 2.valvis, valv. inferiore subcoriacea subulata 3-quetra superiorem membranaceam carinatam includente, Car. valvulis adnata.

1. N. stricta: spica erecta secunda. Schr g. I. 157. A. 3-6" 24. Rad. squamosa. Fol. subulata. Fl. 5, 6, 7. In solo arenoso caespites sparsos undique fere formans. Hasenh. Jusngfh. etc.

\section{ORDO II. DIGYNIA.}

\section{IEERSA.}

Cal. o. Cor. 2-valv. coriaceo-membranacea carinato-compressa clausa, valv. superiore angusta, inferiore semiovali. Car. libera valy. tecta. (Ind. panicula.)

1. L. orizoides (Sirariz): pan. diffusa, ramis fleruosis, fosc. 3-andris, spiculis semioralibus ciliatis. Mert. g. I. 493 .

Asprella orizcides. L. R. S. II. 266: Phalaris oryzoides $L$.

A. 1-4' 24. Rad. sobolifera. Fol. linearia. Fl. S, o. In pratis et locis paludosis. Ghax. lottb. Kth. Teg. Rummelsb. S.

26. ALOPECURUS.

Cal. 2-valv. 1-florus, valv. basi coriatis aequalibus. Cor, 1-valvis, vâtv. in utriculum conna- 
tis et latere uno fissis in dorso aristatis. Car. elliptica tecta. (Infl. pan. spiciformis.)

1. A. pratensis : culmo erecto glabro, pan. cylin. dracea obtusa, ramis 4-6-floris, glumis sub medio connatis, valv. dorso piloso-ciliatis. Mert. g. I. 478 .

a. minor. B. major. S. I. 39. A. $\frac{1}{2}-4^{\prime} 4$. Fol. linearia. $\mathrm{Fl}, 5-9$. In locis graminosis pinguioribus undique frequens.

2. A. geniculatus: culmo glabro basi procum. bente adscendente, pan. cylindracea spicato-contracta, spiculis ovato-oblongis, ralv. obtusis ciliatis basi connatis, gluma sub medio aristata. Mert. g. I. 480 .

a. aquaticus. f. terrestris. S. I. 39. A. $\frac{r}{4}-1 \frac{1}{2}{ }^{\circ}$. Fol. linearia. FI. 5-9. Frequentissimus in. cola soli humidi.

\section{PHLEUM.}

Cal. 2-valvis 1 -florus flosc. longior, valv. subaequalibus carinato-compressis truncatis $\nabla$. acuminatis, carina aristata v. mucronata. Cor. 2valvis, valv. membranaceis dorso aristatis v. muticis. Rudimentum flosc. superioris pedicel. liforme v. o. Car. tecta. (Inf. pan. spiciformis.) - Rudiment. flosc. o. Spiculae oblongae. (Phleum P. Beauvois.) 
1. P. pratense: racemo spicato cylindraceo, $\nabla$ alv. oblongis truncatis mucronato-aristatis carina hispido-ciliatis, aristis cal. brevioribus. Mert. g. I. 489. a. altum. $\beta$. humile. S. A. 1-4' 4. Rad. fibrosa. Fol. linearia. Fl. 5-9. In pratis, syl. vis etc. undique.

* Rudiment. flosc superioris. Spicularum forma diversa. (Chilochloa P. Beauvois.)

2. P. Boehmeri (Wibel): pan. spicata cylindracea, valv. lineari-oblongis apice rotundato-truncatis, breviter aristatis carina scabriusculis v. hispidociliatis. Mert. g. I. 49 f.

Chilochloa Boehmeri. S. I. 41. L. R. S. II. 408. A. $\frac{1}{2}-2 \cdot 2$. Rad. fibrosa. Fol. linearia Fl. $5-7$. In campis siccioribus herbidis, Strilll. Hasenh. etc.

\section{S. PHAIAIS.}

Cal. 2-valv. 1-Horıs, valv. subaequalib, carinatocompress, corollae valv. longioribus. Cor.2-valvis, valv. coriaceo.induratis, caryopses cortican. tibus. Rudim. 2 squamaeformia pilosa adpressa in fundo flosculi. Car. corticata. (Infl. pan.)

1. $P$. arundinacea: pan. patente glomerata, rudi. mentis sericeo-pilosis, valvulis nitidis. S. I. 41. Arundo colorata.

A. 2-6'24. Rad. repens. Fol. lanceolato-li- 
ria. Fl. 6, 7. In locis irriguis frequentissime conspicua planta.

29. MIIIUM.

Cal. 2-valv. 1-florus, valv. convexo-ventricosis. Cor. 2-valvis, valv. demum cartilagineis oblongo-concavis muticis $v$. ex apice aristatis, arista basi contracta decidua. Car. subbicornis corolla tecta. (Inf. pan.)

1. M. effusum: pan. diffusa, for muticis ovatis disper'sis, rad. repente. S. I. 42.

A. $\frac{\mathrm{I}}{4}-1 \cdot 2$. Fol. lanceclata. Fl 5,6. In locis humidis umbrosis gregatim. Thiergart. Papenb. etc.

30. AGROSTIS.

Cal. 2-valv, 1.florus convexo-compressus muticus flosc. longior. Cor. 2-valv. membranacea mutica $\nabla$. aristata, arista gracili, basi pilorum fasciculis stipata 4 parte longitudinis brevioribus; valv. superiore minore interdum J. Rudim. flosc. superioris pedicelliforme v. o. Car. libera tecta. (Inf. pan.)

* Valv. inferiore breviore, rudion. flosc. superioris, spiculis longe aristatis. (Anemagrostis Trinius:)

1. A. Spica venti: pan. diffusa multifiora, flosc. sub apice aristatis, arista recta valv, multipliciter v. multo longiore, antheris lineari-oblongis. Mert. g. I. 50 f. 
A. $\frac{1}{2}-3^{\prime} \odot$. Fol. linearia, scabra. F1. $5-\delta$. In agris ubivis conspicua planta.

- Valv. inferiore longiore, rudim. o., fol. planis. (I ilia. P. Beauv.)

2. A. vulgaris: pan. diffusae ramis hispidnlis, fol. planis, ligula truncata, flosc. 2-valv. valv. inferiore mutica v. brevius aristata, rad. sobolifera. S. I. 43 .

Agrostis alba, stolonifera, capillaris, hispida, rwbra, compressa, vinealis. rar. Auct.

a. laxa. fo. rigida. $S, l . l$. A. $1-3 \cdot 24$. F1. 7, 8. Rad. stolonifera. In solo sicciore ez humidiore frequenter obria planta polymor. plia, quam ob rem jure conjungit $S$. species supra enumeratas.

* Talv. inferiore longiore, rudim. flosc. o, valv. superiore plerumque o. (fol. rad. angustis $v$. setaceis). (Trichodium. Michaux. P. Beauv. Schrader,)

3. A. canina: pan. ovata diffusa, ramulis squarrosis scabriusculis, valv. ovato-lanceolatis acutis, flosc. sub medio aristatis, fol, radicalibus setaceis. Mert. g. I. 512 . S. I. 45.

Trichodium caninum. Schr. g. 1.. 198.

a. sylvatica $\beta$. arida. S. l. l. A. 1-2, 4 . Rad. sobolifera. Fl. 6, 7. In campis et sylvis Jungfb. Grunw, Teg. etc, copiose. 
31. ARUNDO.

Cal. 2-valv. 1-flor, valv. convexo-compressis muticis cor. valv. superantibus. Cor. valv. 2 membranaceae basi pilis cinctae, inferiore mutica v. aristata, superiore 2-carinata. Rudimentum flosc. (penicillatum) 1. v. o. Car. libera, tecta. (Infl. pan.)

* Tals. inferiore longiore, glumis membranaceis, rudim. 0 .

3. A. Epigeios: pan. stricta patente glomeratolaciniata, valv. lanceolatis apice compresso-subulatis, pilis cor. longioribus, arista recta e medio proveniente. Mert. g. I. 520.

A. $2-6 \cdot 2$. Rad. repens, Fol. sublanceolata. Fl. 6, 7. In campis, sylvis et collibus arenosis gregatim. Teg. Thierg. etc.

2. A. Calamagrostis: pan. diffusa, valv. angustelanceolatis acuminatis, pilis cor. longioríbus, arista terminali subapicali brevi. Mert. g. I. 518 .

Agrostis lanceolata. L. R. S.

A. $2-4^{\circ} 2$. F1.6. In sylvis humidis et in ripis inter frutices sparsim frequens; Thierg. Grunw. Strahl. etc

* V'alv. inferiore longiore, glumis coriaceis coloratis, rudim. penicilliformi.

3. A. stricta (Timm., : pan, angusta patente rigi- 
do.erecta, valv. acutis, pilis cor. brevioribus, arista recta sub medio dorso. Mert. g. I. 522.

A. 2-3' Rad, repens, Fol. acuminata. Fl. 6, 7. In pratis turfusis sparsim. Jungfernh, Grunw. Spand.

4. A. sylvatica (Schrader): pan, angusta patente, glumis acutis pilis brevissimis (cur. 4-plo brevioribus), arista dorsali geniculata glumas excedente Sclsr. g. I. 218. Mert. g. J. $52 \div$

A. $2-4^{\prime}$ 24. Rad. repens. Fol. linearia, acn1a. Fl. 6, 7. In sylvarum solo sicciore passim. Papenb. Köpn Ileide etc.

* * Valv. inferiore breviore, glumis coriaceis, rudin. penicilliformi. (Psamma. L. R. S.)

5. A. arenaria: pan. subspicata contracta cylindrica supra attenuata, valv. lineari-lanceolatis acutis, pilis cor. 3-plo brevioribus, arista subapicali brerissima, fol. involutis. Mert. g. I. $52 j$. S. 1.48 A. $2-3^{\prime} 24$. Rad, repens. Fol. acuminata. Fl. 7, 8. In arenosis sterilibus. Spand, Kth. Zwischen Reineekend. u. Hermsd, S.

$$
\text { 32. } \operatorname{sTrPA} \text {. }
$$

Cal. 2-valv. 1.florus convexo-compressus flosc. longior: valv. acutissimis v, aristatis. Flosc. 2 -valv. nervo medio apice incrassato v. noduloзо et in aristam reetam elongato. Car. cor. 
valv. tecta libera. (Inf, pan.) (Cor, valv, extrinsecus non basi pilosae.)

1. S. pennata: pan. basi inclusa, aristis longe geniculatis plumosis a basi usque ad 4 longitudinis partem nudis. Mert. g, I. 531.

A. $1-3^{\prime} 24$. Rad. caespitosa. Fol. linearia rigida. Fl. 5,6 . In collibus aridis. Spand. Bamberge.

2. S. capillata: pan. basi inclusa, aristis longissimis geniculatis glabris. Mert. g. I. 532.

A. $1-2,24$. Rad. caespitosa. Fl. 6, 7. In collibuz siccis. Kalkb.

\section{A VENA.}

Cal. valv. 2 inaequales 2 - multiflorae. Flose. ఫ̧. Cor. valv, 2 saepius basi barbatae, inferior apice dentata 2 -fida $\nabla$. 2 -aristata arista tortili vaga sub $\nabla$. supra dorsi medium oriente. Car. corticata. (Infl. pan.)

* Spiculis post effloresc. declinatis, ca'. 5-9-nerviis, germine piloso-comoso.

1. A. sativa: pan. aequali patente, spiculis sub2 - floris, flosc. calyce minoribus nudis lanceolatis supra attenuatis apice 2 - fidis denticulatis, sıperiore mutico glabro, axi glabro in fundo flosc. inferioris breviter fasciculato.piloso, Mert.g. J.556. g. I. 556 .

๙. aristata. $\beta$. mutica. S. I. 53. 
A. 1-4 $4^{\circ} \odot$. Fl. 7,8 . Planta frequentissime in agris culta.

2. A. orientalis (Schreber): pan. secunda contracta, cal. subbifloris flosc. longioribus, flosc. nudis lanceolatis supra attenuatis apice 2-fidis denticulatis, superiore mutico, axi nudo in fundo llosc. inferioris subpiloso. Mert. g. I. 557 .

A. 2- $4^{\prime} \odot$. Fl. 7, 8. Planta praec. similis his illisve locis culta.

3. A. strigosa (Schreber): pan. subsecunda, cal. subbifloris flosc. longitudine, flosc. nudis lanceolatis supra attenuatis apice bifido-aristatis, utraque arista dorsali instructa, axi glabro, superioris flosc. fundo breviter fasciculato-piloso. Mert. I. g. 557. Danthonia strigosa. L. R. S.

A. 2-3' $\odot$. Fl. 17,8. Inter segetes Arenae sativae. Thierg. Schöneb. passim. S.

4. A. fatua: pan. aequali diffusa, cal. sub-3-\$lo. ris, Hosc. basi et in dorso setaceo-pilosis lanceola tis supra attenuatis apice denticulato-2-fidis aristatis, axi piloso. Nert. g. I. 559 .

A. $2-4^{\prime} \odot$. Fl. 7, 8. Inter segetes plerumque inter secale frequens. IVillmersd. Kils.

* Cal. 1-3 nervi compresso, germine glabro, arista dorsali e medio vel supra oriente.

5. A. favescens: pan. aequali, ramulis $5-8$ spi- 
culas gerentes, spiculis subtriflor is, axi villoso, valv. superiore oblonga lanceolata acuminata, flosc. bascos pilis multo longioribus, segmentis apicalibus cuspidatis, culmis plerumque simplicibus, fol. pla. nis. Mert. g. I. 562 .

Trisetum flavescens, L. R. S. II. 663.

A. 1-2'24. Tad. subrepens. Fl. 7-0. In pratis pascuis et nemoribus graminosis hinc inde. Thierg. Hasenh, etc.

** Cal, 1 - 3 nervi semitereti-convexo, germine compresso, arista dorsali in medio dorso vel supra medium oriente, germine villoso.

6. A. pubescens: pan, aequali subracemosa, ramis subternis spiculis 3 -horis, axi vilioso, flosc. baseos pilis multipliciter longioribus, apicis segmentis inaequaliter dcnticulatis, fol. planis, culmo tereti erecto, Mert. g. I. $56 \mathrm{~s}$.

A. $1 \frac{\mathrm{r}}{2}-3 \cdot 24$. FI. 5, 6. Rad. sub sobolifera. In sylvis et pratis gregatim frequens. Friedrf. Jungfh. Chan lottenib. etc.

7. A. pratensis: pan. contracta racemosa, ramis $1-2$ scaberrimis, spiculis subquinquefloris, axi villoso, flosc. baseos pilis multiplo longioribus, fol. convolutis v. planis margine scaberimis, culmo tereti. Mert. g. I. 569.

A. 1-3. 4 . Rad. fibrosa. Fl. 6, 7. Acerva- 
tim in agris et collibus siccis. Fuchsb. Rollb. Reliberge.

*** Cal.1-3-nervi, arista dorsali sub medio $v$ supra basin oriente, germine nudo. (Aira Schrader.)

\&. A. flexuosa: pan. patente, spiculis 2 -floris, fosc. apice 4-dentatis supra basin aristatis cal. longitudine, fol. setaceis. Mert. g. 570. S. I. 55.

Aira flexuosa. Schr. g. I. 250.

A. $\frac{1}{2}-2$ 2 . FI. 5, 6, 7. Rad. fibrosa. In pascuis et sylvis siccis graminosis frequens. Grunw. IIasenh. etc.

9. A. caryopliyllea (Weber): pan. divaricata, spiculis 2-floris, flosc. apice bifido-cuspidatis sub medio aristatis cal. brevioribus, fol. setaceis. Mert. g. I. 572. S. I. 55 .

Aira caryophyllea. Schr. g. J. 261.

A. 4-12" ○. Fl. 5, 6, 7. Rad. fibrosa. In collibus et agris argillaceo-arenosis hinc inde copiosa. Hasenh. Kth. Weissens.

10. A. praecox (P. Beauv.): pan. spicato-contracta, spiculis 2 -floris, cal, apice bifido-cuspidato sub medio aristato flosc. breviore, fol. setaceis. Mert. g. J. 573 . S. I. 56.

Aira praccox Schr. g. I. 268.

A. $\frac{x}{2}-6 " \bigodot$. Panicula virescens. Fl. 5, 6. In locis arenos. Jungfh, versus Spand, sparsim. Kth. 


\section{TRIODIA.}

Cal. 3-5-hor. 2-valv. valv. magnis convexodilatatis flosc. cingentibus. Cor, valv. 2 basi bar. batae, inferior apice 2-fida 3-dentata interjecto mucrone, supetior 2.carinata. Car. libera. (Infl. pan.)

3. $T$. decumbens (P. Beauv.): pan. racemosa, ramulis solitariis, inferioribus sub 2 -floris, spiculis oblongo-ovatis $3-5$-floris, fol. planis, ligulis pilosis, culmo florifero erecto. Mert, g. I. 675. S. I.5C. Poa decumbens. Schr. g. 1. 305. Festuca decumbens $W$.

A. 1-1?' 4. F1. 6, 7. Pad, subrepens. In locis graminosis et sylvis haud rara. Thierg. Grunw. Jungfh. etc.

35. BROMUS.

Cal. 2-valv. 3- muliflorus, Car. 2-valv., valv. inferiore apice emarginata bimucronata $v$. biaristata, arista recta v. reflexa sub apice oriente, superiore in carina ciliis remotis rigidis setaceis pectinata. Car, adnata. (In⿴. pan.)

* Valv. concavis superiore 5-polynervi, spiculis oblongis $v$. lanceolatis superne non latioribus.

1. B. secalinus: pan. patente, fructifera nutante, spiculis oblongis, flosc. late ellipticis margine re* 
curvato teretibus distinctis, aristis undulatis glumis brevioribus, ligulis glabris. Mert. g. J. 679. A. $1 \frac{\mathrm{r}}{2}-3^{\prime} \odot$. Fl. 6, 7. Inter segetes undique frequens.

2. B. racemosus: pan. erecta v. subnutante post inflorescentiam contracta, spiculis ovato-oblongis glabris, flosc. late-ellipticis margine rotundatis, maturitate invicem imbricatis, aristis rectis valv. subaequantibus; fol. et ligulis inferioribus pilosis. Mert. g. I. $68 \mathrm{r}$.

A. 1-2' $\odot$ v. $0^{\top}$. Fl. 5, 6. In locis humidis graminosis. Thierg. Fasanerie.

3. B. mollis: pan. erecta post infloresc. contracta, spiculis ovato-oblongis pubescentibus v. glabris et scabriusculis, flosc. Late-ellipticis margine recto, fructiferis imbricatis, aristis rectis valv. subaequantibus, fol. ligulisque infer, pilosis. Mert. g. I. 682. \%. procera. i3. congesta. $\gamma$, nana. A, $\frac{r}{7}-1$ ' $\delta^{7}$.

Fl. 5, 6. In locis siccis et hmmidioribus undique frequens,

4. B. commutatus (Schrader): pan. laxa patente, fructifera nutante, spiculis oblongo-lanceolatis glabris, flosc. rhombeo.ellipticis margine recto, fructiferis imbricatis, aristis rectis valv. longioribus, fol. ligulisque pilosis. Mert. g. J. 093.

A. $1^{\frac{T}{2}}-3^{\circ} 0^{7}$. Fl. 5, 6. Inter segetes ad rias sepesque passim $\mathrm{Kth}$.

F. $B$. 
5. B. arvensis: pan. patente erecta, fructifera sub. nutante, spiculis lincari-lanceolatis, flosc. ellipticolanceolatis margine obtuse prominentibus, aristis rectis $\nabla$ alv. longitudine, fol. ligulisque pilosis. Mert. g. I. 686.

A. $1 \frac{1}{2}-3^{\circ} \odot$. Fl. 6, 7. In agris ad vias et inter segetes copiosus. Charlottb. etc.

- Valv. carinato-compressis, inferiore 1-, superiore 3-nervi, spiculis oblongis superne latioribus.

6. B. sterilis: pan. laxa demum nutante, ramulis elongatis apice nutantibus, spiculis oblongis superne latioribus, flosc. lanceolato-subulatis, aristis rectis valv. longioribus, culmo glabro. Mert. g. I. 688 .

A. $1-2^{\prime} \odot$. Fl. $5-8$. In arvis et agris satis copiosus. Thierg. Jungfh. etc.

7. B. tectorum: pan. nutante subsecunda, spiculis linearibus, flosc. lanceolato-subulatis, aristis rectis valv. subaequantibus, culmo superne pubescente. Mert. g. 1. 6ss.

A. $\frac{1}{2}-1 \frac{1}{2}{ }^{\prime} \odot$. Fl. 5, 6. In solo sicco, in muris, tectis etc. frequentissimus.

36. FESTUCA.

Cal. 2-valv., 2-multiflor. Hosc. brevior. Flosc. lanceolati v. lanceolato-subulati, dorso ro- 
tundato. Cor. 2-valv. valv. inferiore apice integra $\nabla$. 2-dentata mutica $\nabla$. ex $v$. sub apice aristata, superioris carinis dense et tenere ciliatis. Spiculae ebracteatae. Car. libera v. adnata. (Infl. pan.)

* Rad. - fol. rad. non permanentibus, pedunculis superne incrassatis, cal. inaequali valv. inferiore saepe brevissima, flosc. 1-andris lanceolato-subulatis aristatis. (Mygalurus. Link. Enum. Hort. Berol. 1. 92.)

1. F.Myurus: pan. secunda spicato-contracta elongata subnutante, flosc. 1-andris apice scabris arista brevioribus, culmo usque ad pan. ligulis tecto. Mert. g. I. $637 . \quad$ S. I. 68 .

Mygalurus caudatus. Link, l. l. A. I-2 $\sigma^{\prime}$ v. $\odot$. Fl. 5, 6. Sparsim in agris et ad latera lacuum Schönh. Kth. Temph. W. S.1.1.

* Rad. 24 . fol. rad. permanentibus, flosc. lanceolatis acuminatis muticis $v$ arista. tis, valv. inferiore margine membranacea.

2. F. ovina: pan. contracta angusta erecta, spiculis oblongis breviter aristatis v. muticis plerumque 4-floris, fol. capillaribus scabriusculis v. scabris, ligulis 2-auritis. Mert. g. I. 641.

A. $1-1 \frac{1}{2}, 2$. Fl. 5,6 . In arvis sylvisque siccis frequens. Hasenb. Thierg. etc. 
3. F. duriuscula: pan. patente, spiculis oblongis plerumque 5 -floris aristatis, fol, setaceis scabriusculis v. scabris, ligulis 2-auritis. Mert. g. I. 646.

๙. leiantha. $\beta$. dasyantha. S, I. 67. A. $1-2^{\prime} 24$. Fl. 5, 6. In graminosis siccis et in sylvis copiosa.

4. F. 'glauca (Schrader): pan. patente, spiculis oblongis plerumque 5 -floris aristatis, fol. setaceis glabris rigidis, ligulis 2-auritis. Mert. g. I. 649.

A. $1-1 \frac{1}{2} \cdot 24 . \mathrm{Fl} .5-8$. In locis arenosis $e t$ sylvis acerosis undique obvia.

5. F. rubra: pan. patenie, spiculis oblongis subquinquefloris aristatis, flosc. lanceolato-acuminatis (arista lorgioribus), fol. radicalibus setaceis, fol. culmeis planis $r$. convolutis, ligulis biauritis, rad. repente caespites formante. Mert. g. I. 653. S. I.67. A. $1-2+24.11,5-5$. In locis siccis graminosis frequerter obvia.

** Rad. 24. fol. rad. et culmi linearilanceolatis, flosc. lanceolato-acuminatis $v$. obtusiusculis, muticis $\vee$. sub apice aristatis.

6. F. elatior: pan. difiusa nutante, ram. ramosis inferne geminis, spiculis ovali-lanceolatis 4-5-floris, flosc. sub apice mucronatis v. muticis, fol. planis lanceolato-linearibus, ligulis brerissimig. Mert. g. I. 665 . 
Bromus elatior. S. I. 63. Schedonorus elatior. $P$. Beauv.

A. 2-6, 24. Fl. 5, 6, 7. Pad. subrepens. In sylvis et pratis humidis haud infrequens.

7. F. pratensis (Hudson): pan. subsecunda erecta patente, ramis subgemin. simplicib. racemosis, spicul. linearibus 5-10-foris, flosc. sub apice mucronatis v. inermibus, fol. planis lanceolato-linearibus, ligulis brevissimis. Mert. g. I. 665.

Bromus pratensis. S. I. 63. Schedonorus. pr.L.R.S. A. 1-3، 24. F1. 6-8. Rad. fibrosa. Planta vulgaris in graminosis siccis et humidis.

8. F. gigantea: pan. laxa patentissima, ram, apice nutantibus, spiculis lanceolatis 5-8-floris, flosc. sub apice aristatis, aristis flexnosis valv. duplo longioribus, fol. lanceolato-linearibus planis gla. bris, ligulis brevissimis. Mert. g. I. 671.

Bromus giganteus L. S. I. $57^{\circ}$ A. $3-4^{\circ} 4$. F1. 6, 7, 8. Rad. fibrosil subrepens. In sylvis frondosis. Thierg. Fapenb. Grunw. etc.

9. F. montana (Savi): pan. aequali erecta ramis inferioribus 3-nis 6-nisve, spiculis lineari-lanceolatis 5-10-floris, flosc. sub apice aristatis (imbri. catis), aristis dimidiae valv. longitudine, fol. pla. uis, radicalibus angustis ciliatis, ligula brevi. Mert. 3. I. 673 .

Bromus erectis Hudson. S. I. 62. A. $1 \frac{1}{2}-2 \cdot 24$. Fl.6,7. Rarius in sublumidis sylvar. Thierg, $S$. 
10. F. inermis (De Candolle): Ipan. aequali erecta, ranulis $4-6-n i s$, spiculis lineari-lanceolatis $5-10$ floris, flosc. muticis $\nabla$. sub apice breviter aristatis, fol. linearibus glabris planis, ligula brevi. Mert, g. I. 674 .

Bromus inermis Schr.g. I. 359. S. I. 62. Schedonorus inermis. $L, R$. $S$.

A. $2-4^{\prime} 24$. Fl. 6,7 . In solo meliore in agris, pratis, ad sepes Weilss. Friedrf, etc.

$$
\text { 37. AIRA, }
$$

Cal. 2-valv. 2-flor, v, 2-flor, c. rudimento flosc. 3. Flosculi ఫ̧. basi pilosi. Cor. 2-valv., valv. inferiore arista recta e basi v, medio dorso oriente instructa, (Inf, pan.)

* Arista setacea. (Deschampsia P. Beauv.) 1. A. caespitosa: fol. planis, fpan. diffusa, pendunculis scabris, arista setacea valv. subaequante. Mert. g. I. 552.

Deschampsia caespitosa. P. Beauv. S. I. 51.

u. major. B. minor. S. l.l. A. 2-4.24. Rad. caespitosa. Fol. plana, sulcata. FI. $6-8$. In pratis, sylvis, locis humidis etc. caespitosim frequens.

* Arista supra medium clavata. (Corynephorus P. Beauv.)

2. A. canescens: fol. setaceo-complicatis, pan. contracta patente, (flosc, aristaque glumis minori- 
bus), arista supra medium clavata. Mert. g. I. 554 . S. I. 50 .

Corynephorus canescens. P. Beauv. S. l. l.

A. $\frac{r}{2}-1^{\prime}$ 24. Rad. caespitosa. Fol. setacea. FI. $6-8$. In campis collibus et sylvis caespi. tosim frequens.

38. KOELERIA.

Cal. 2-valv. 2-5-forus, valv. carinato-compressus, fosc. subaequintibus. Flosc. sublanceolati valde compressi recti. Cor. 2-valvis, valv. inferiore mutica $\mathrm{v}$. breviter mucronata $\nabla$. sub apice aristata, superiore in carinis dense et tenere ciliata. Car. libera tecta. (Infl, pan, sub. spicata contracta.)

1. $K$ cristata (Persoon) : pan. spicata basi interrupta, spiculis 3 -4-horis, flosc. acutis v. obtusiusculis demum plerumque mucronatis, fol. planis villoso-ciliatis v. glabris. Mert. g. I. 580.

Aira cristata et A. glauca. Schr. g. I. 255. S. I. 64. 65.

๙. ciliata. $\beta$. glabra. A. $\frac{1}{2}-1 \frac{1}{2}{ }^{\prime} 4$. Rad. caespitosa. Fl 6, 7. In collibus sylvis et arvis arenosis frequens.

Conjunximus Airam cristatam et Airam glaus cam Schrader exemplo Cel. Mertens et Koch, plantas sibi invicem simillimas. 


\section{DONAx.}

Cal. 2-valv. Alosc. subaequaus. valv, lanceolatis.

Cor. 2-valvis, valv. lanceolat. dorso rotundat. basi barbatis, infer. apice 3-5-cuspidata, superiore subconduplicata. Car. adnata. (Infl. panicula.)

1. D. festucaceus (P. Beauv.): spiculis sub-4-Aloris, flosc. glumas calycis parum excedentibus, culno ramoso, ligula oblonga, rad. repente. S. J. $6 \delta$. Arundo festucacea IV. Festucu borealis. Mert.g. I.664. A. $3-4^{\prime} 2$. Fl. 7, S. In fluviis et lacubus prope ripas satis frequens. Teg. Strall. Trept. 40. DACTYIIS.

Cal. 2-valv. 2-multiflorus compressus flosc. brevior. Flosc. lanceolati dorso carinato-compressi apice incuryato. Cor. 2-valvis, valv. inferiore carinata apice emarginata mucronata, superiore subconduplicata in carinis tenere et dense ciliata. Car. adnata. (Inf. panicula glomerata secunda.)

1. D. glomerata: pan. secunda glomerata, ramis basi nudis, spiculis subtrifloris, fol. carinatis. Mer. g. I. 634 .

a. vulgaris, $\beta$. collina. S. I. 69 . A. 1-3.24. In locis herbosis, pratis siccioribus et sylvis frondosis vulgaris.

41. CYNOSURS.

Cal. 2-valv.3-5-florus, valv. membranaceaeflosc. bre- 
viores. Cor. valv. 2 inferior apice rostrato-aristata, superior subconduplicata. Spicula quae. vis involucro e glumis alternis numerosis di. stichis composito sufulta. Car. tecta. (InI. pan. coarctata secunda.)

1. C. cristatus: pan, coarctata spicaeformi secun. da erecta, involucro unico spicularum (glumis muticis), fol. linearibus marginec arinaque scabris S. I, 70 .

A. $1-2,24$. Fl. 5, 6. In pratis ot pascuis undique obvius.

$$
\text { 42. BRIZA. }
$$

Cal. val. 2 inflatae ovato.cordatae fosc. breviores. Cor. valv. 2 ventricosae basi cordato-auriculatae muticae. Car. orbicularis 2 -rostrata tecta. (Infl. panicula.)

1. B. media: pan. erecta patente, spiculis subro. tundis 5 - 9 floris, cal. valv. flosc. proxime se. quentibus brevioribus. Mert. g. I. 622.

A. $\frac{1}{2}-1 \cdot 24$. FI. 5. 6. In: pratis pascuis, sylvis et collibus ubivis conspicua.

$$
\text { 43. GI Y CERI } \Lambda \text {. }
$$

Cal. 2 -valv. 2 -multiflorus convexus. Flosc. ob. longi obtusi $v$. truncati intus subventricosi dorso rotundato. Cor, 2-valv, mutica. (InA. panicula.). 
1. G. fluitans ( $R$. Brown): pan, secunda divaricata, spiculis lineaibus appressis 7-11 Horis, flosc. obtusis liberis 7-nervibus (nervis prominulis), rad. repente. Schr. g. I. 2 So. S. I. 77.

Festuca fluitans W. Poa fluitans. Schr. l. l.

A. 2-4' 4 . Fl, 6-8. In paludibus fossis ad lacuum fluminumque ripas frequens.

2. G. distans (Wahlenberg): pan, aequali divaricata, ramis fructiferis deflexis, spiculis linearibus 4-6-floris, flosc. obtusis obsolete 5 -nervibus, rad. fibrosa. Schr, g. I. 282. Mert. g. I. 588.

Poa distans. Schr. l. l. S. I. 71 .

A. $1-1 \frac{\mathrm{I}}{2} \cdot 24 . \mathrm{Fl} .5,6$. Ad ripas Spreae et in fimetis rarius. Stadt, vor dem Frankfurther und Köpnicker Thor, vor dem Strahlower und Hallischen,

3. G. aquatica (Presl.): pan, aequali diffusa, spiculis linearibus plerumque 2 -floris, flosc, obtusis (glumas excedentibus) 3-nervibus, nervis prominulis, rad. repente. Mert. g. I. $58 \mathrm{~g}$.

Aira aquatica, Schr.g.1. 256. Catabrosa aq. S.1.78.

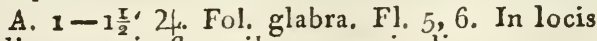
udis et aquis fuentibus, e. c. in ligno natan. te Spreae frequens.

4. G. spectabilis (Mert. et Koch): pan, aequali ramosissima diffusa, spiculis linearibus $5-9-$ Horis 
losc. obtusis 7 -nervibus, nervis prominentibus, rad. repente. Mert, g. I. 586.

Poa aquatica Schr. g. I. 278 . S. I. 71.

A. 4-8'24. Fol. lineari-lanceolata basi 2. maculata. Fl. 7,8 . Acervatim ad fluminum ripas, in fossis et paludibus.

$$
\text { 44. POA. }
$$

Cal. 2-valv. 3.multiflorus compressus. Flosc, ova-

ti v. lanceolati recti dorso carinato-compressi, rarius intus ventricosi. Cor. 2-valv. valv. inferiore apice acuta $v$. obtusa mutica v. mncronata, superiore in carinis dense et tenere ciliata. (Infl, pan.)

1. $P$. annua: pan. subsecunda divaricata, ramis glabris solitariis $\mathrm{v}$. geminatis fructiferis reflexis, spiculis oblongo-ovatis $3-7$-floris, ligulis superioribus oblongis, culmis eompressis basi interdum radicantibus, rad. fibrosa. Mert. g. I. 598.
A. $\frac{\mathrm{T}}{2}-1^{\prime}$
$\odot$ et $\sigma^{7}$. Fl. 4-9.
Ubique fre. quens.

2. $P$. compressa: pan, subsecunda, ramis geminatis v. quinatis, spiculis oblongo-oratis $5-9$-lloris, flosc. obsolete-nervibus basi pilosis, culmo ancipiti compresso basi procumbente, rad. repente. Mert. g. I. 621 .

A. $\frac{1}{2}-1 \frac{1}{2} \cdot 24$. Fl. $5-9$. In agris, ad vias copiosissime. 
3, P. bulbosa: pan. aequali orata patente, ramis geminatis scabriusculis, spiculis ovatis 4-7-foris, culmo supra rad. tumido, ligulis superioribus oblongis, rad. fibrosa. Mert. g. I. 6og. S. I. 73.

A. $\frac{1}{2}-1 \cdot 2$. Fl. 5, 6. Flores plerumque vivipari. In solo arenoso sylvarum etc. frequens.

4. $P$. trivialis: pan. aequali diffusa, ramis subquinis scabris, spiculis ovatis subtrifloris, !losc. nervosis basi subpilosis, culmis ligulisque scabris, ligula superiore acuta, rad. fibrosa. Mert. g. I.611. S. I. 75 .

A. 2-4' 4 , Fol. scabriuscula. Fl. 5-9. In graminosis frequens.

5. $P$. pratensis: pan. aequali diffusa, ramis sca. bris subquinis, spiculis ovatis $3-5$-floris, flosc. distincte-ncrvibus basi pilosis, culmis ligulisque glabris, ligula brevi truncata, rad, repente. Mert. g. I. 612 .

a. latifolia. B. angustifolia. $\%$. convolutifolia. S. i. 7 f. A. $\frac{1}{2}-3^{\prime} 4$. Fl. 5,6 . In locis subhumidis, in pratis etc. frequells.

6. $P$. nemoralis: pan. aequali $\nabla$. subsecunda, ramis scabris geminatis ad quinatos, spiculis elliptico-lanceolatis 2-5-Aoris, flosc. obsolete-5-nervibus basi pilosis, culmis ligulisque glabris, ligulis brevissimis v. o., rad. subrepente. Mert. g. I. 615.

Pog serotina Ehrhardt. S. 1. 75. 
A. $\frac{1}{2}-3^{\prime} 24$. Planta polymorplia in locis humidiusculis et in sylvis frequentissima.

Observationibus Mertensii et Kochii alio. rumque Botanicorum adducti satius habuimus conjungere Puam servinam Ehrhdt. cuma Poa serotina $L$.

45. MOIINA.

Cal. 2-valv, 2-4-florus convexus. Flosc. latere interiore supra basin rentricosi dein conici $r$. conico-subulati dorso recto rotundato. Cor. 2-valv. valv. inferiore apice integra nutica $v$. mucronata, superiore in carinis dense et tonere ciliata. Car. 2-mucronata. (Infl. pan.)

1. M. coerulea (Koeler): pan. subcontracta, spiculis erectis muticis subtrifloris, flosc. supra conicis 3.nervibus, culmo subnudo. Mert. g. I. 583. S. I. 77 .

Enodium coeruleum. L. R. S. II. 531. Melica coorulea. Schr. g. I. 269 .

œ. pratensis: fo. syluestris. S. A. $1 \frac{1}{2}-2 \frac{x}{2}, 24$. F1. 6,7 . In locis humidiusculis, pratis, sylvis etc. frequens.

46. MEXICA.

Cal. valv. 2 magnae membranaceae ovatae flosc. nequantes I-2-Alare. Flosc. inferiores I-2 perfecti $\zeta$ cor. valv. 2 coriaceo-membranace is muticis. superiores incompleti $\hbar$ (inclusi) 
valv. truncatis. Car. libera nitida. (Inf. pan. secunda.)

1. M. nutans: cor. valv. inferiore imberbi, pan. subsimplici contracta secunda, spiculis nutantibus ovatis 3-floris, flosc. tertio imperfecto. Schr. g. I. 267. S. 1. 79 .

A. $1-2$ ' 24, Fl. 5, 6. Rad. repens. In sylvis frondosis loumidis. Thierg. Papenb. Teg. Köpn.Haide.

2. M. uniflora (Retz): cor. valv. inferiore glabra, pan. racemosa laxa secunda, ramis patentibus, spiculis erectis ovatis, Hosc. I perfecto. Mert. g. I. $577^{\circ}$ S. I. 79.1

A. 1-2, 24. Fl. 5, 6. In sylvis frondosis. Papenb. S.

47. PHRAGMITES。

Cal. valv. 2 flosc. breviores 3-7-florae. Flosc. infimus nudus sessilis $\hbar \mathrm{v} \cdot \sigma^{\top}$, reliqui pedicellis sericeo-villosis insidentes ఫ̧. Cor. omnes 2-valv. valv. inferiore elongata acuminata involuta, superiore 2-carinata. Car. tecta. (InH. pan.)

1. $P$. communis (Trinius): pan. nutante ramosissima patula, cal. 5-floris, Mert. g. I. 528.

Arundo Phragmites. L. R. S. Schr. g. I. 223. A. $6-8: 24$. Rad. repens. Fl. 6, 7, 8. In Ia. cubus, fluminibus et stagnis undique. 
48. PANICUM.

Cal. 2-valv. 2-florus valv, inferiore minore $\nabla .0$. Flosc. superior ఫ̧ 2-valv. cartilagineus, inferior $\sigma^{\top}$ v. 古 valv. superiore minore v. o. Spiculae latere uno complanatae, altero eonvexae. Car. corticata. (Infl. pan. variabilis.)

* Spiculis paniculatis diffusis (Panicum. Beauv.)

1. P. miliaceum: pan. laxa nutante, spiculis muticis, fol. lanceolatis pilosis, ligulis pilosis. Mert. g. I. 47 I.

A. 1-3' ○. Fl. 7, 8. Planta his illisve locis culta.

* Pan. spicis 1-lateralibus, valv. aristatis, aristis acutis. (Echinochloa. Beauv.)

2. P. Crus galli: spicis 1.lateralibus alternis op. positisque compositis linearibus, spiculis approximatis aristatis hispidis, rhachi communi basi 5-angulari. Mert. g. I. 467 . S. I. 81.

Echinochloa Crus galli. L. R. S. II. 477.

A. $\frac{T}{2}-1 \frac{T}{2} \cdot \odot$. Fl. 7,8 . Planta in agris vulgatissima.

* * Pan. spicaeformi, flosc. basi setis 2 *. pluribus instructis. (Setaria. Beauv.)

3. P. glaucum: racemo spicato cylindrico, setis sursum scabris, valv. superiore dimidiarum spicu. 
larum longitudine, flosc. $\not$ glumis transversin rugosis. Mert. g. I. 470 , S. J. 82.

Setaria glauca. L. R. S. II. 490 ,

A. $\frac{1}{2}-1 \frac{1}{2}^{\prime} \odot$. Fl. 7,8 . In agris undique conspicuum.

4. $P$. viride: pan. spicata cylindrica, setis sursum scabris, valv. superiore glumas aequante, glumis Hosc. ఫ̧ glabris. Mert. g. I. 469, S. I. 82 .

Setaria viridis. L. R. S. II. 488.

soum.

A. $\frac{\pi}{2}-\mathbf{I}_{2} \cdot \odot$. Fl. 7, 8. In agris copiosissi-

- ** Spiculis in spicas 1-laterales subdigitatas dispositis, valv. muticis, vali. superiore Rlosc. 南o. (Digitaria Scopoli Syntherisma. Schrader.)

5. P. Ischmaeum (Schreber): spicis subternis digitatis', fol. ligulisque glabris, spiculis ellipticis pabescentibus nervis glabris. Mert. g. I. 466. S.J.\$3.

Panicum glabrum. Mert. l.l.

A. $\frac{x}{2}-1 ' \odot$. Il. 7,8 . In locis arenosis undique.

6. $P$. sanguinale: spicis subquinis digitatis patentibus, fol. ligulisque subpilosis, spiculis oblongo-lanceolatis, valv. inferiore flosc. th margine pubescenti nervo Iaterali ultimo nudo. Mert. g. I. 465. S. J. 8 \%

Syntherisma vulgare. Schr. g. I. 16 1. 
A. $\frac{\pi}{2}-1^{\circ} \odot$. Fl. 7,8 . In agris ad vias etc. frequens.

49. ARRHENATERUN.

Cal. 2-valv. 2-florus flosculos subaequans, valv. ovato-lanceolat. convexo.compressis. Flosc. inferior $\sigma^{7} 2$-valvis, valv, inferiore infra medium arista geniculata instructa, superior $ఫ$ 2-valvis, valv. inferiore mutica v. sub apice breviter aristata. Car. adnata. (Inf. pan.)

1. A. elatius (Beauv.): pan. oblonga, flosc. $\not$ su. periore plerumque mutico, $\sigma$ arista curvata, cul. mi geniculis subglabris. Mert. g. I. 546。

$\alpha$. vulgare. (Holcus avenaceus. Schr, g. I. 248. Arrhen. avenaceum. S. I. 85.) 3. bulbosum. (Holcus bulbosus. Schr. g.T.24S. Arrhen. bulbosum. S. l.l. A. $2-3^{\prime} 24$. Fl. 7,8 . In solo meliore e. c. in pra. tis $\alpha$. satis frequens. $\beta$ rarissimum. Teg. Kth.

Conjunximus secundum Mertens et $\mathbf{K o c b}$ Arrhenaterum elatius et bulbosum.

50. HOLCUS.

Cal. 2-valv. 2-florus flosc. longior, valvul. come pressis lanceolat. mucronatis. Flosc. basi pilis cincti, superiore $\sigma^{\top}$, arista recta dein flexa $v$. geniculata supra medium dorsum oriente, inferiore $\Varangle$ mutico. Cor. 2.valvis, valv. inferio. re apice integra. Car. libera. (Inf. pan.)

1. H. lanatus: pan patente, flosc. $\sigma^{\pi}$ arista rellexa cal. inclusa, rad. fibrosa. Mlert. g. I. 550.

F. B.

D 
A. 1-2' 2. Fl. 6, 7. In pratis siccioribus agris etc. undique.

2. H. mollis: pan. patente, flosc. $\sigma$ arista geniculata cal. excedente, rad, repente. Mert. g. 1.55i.

Praecedenti similis, sed rarius obveniens. Rummelsb. Jungfl. S. Papenb.

$$
\text { 51. HIEROCHIOE. }
$$

Cal. 2-valv. 3-florus flosc. aequans, valv. compress. ovatis. Flosc. 2 inferiores $\sigma^{\top} 3$-andri, superior $\not$ 2-andrus. Cor. 2-valves, valv. mu= ticis v. breviter ex apice axistatis. Car. libera. (Inf. pan.)

1. H. Zorealis (P. Beauv.): pan. divaricata, Ipedunculis glabris, spiculis subrotundo-ovatis, flosculorum of valvulis inferioribus ciliatis muticis v. sub apice breviter aristatis. Mert. g. I. 548 .

Hoicus borealis. Schr.g. I. 252.

A. $1-1 \frac{\pi}{2} \cdot 2$. Fl. 5,6 . In pratis rarissime. Prope Ilenningsd, in prato unico. Kth. 52. LOLIU MT.

Ccl. 1-valvis, (in summo flosc. 2-valr.) 3-multiflorus. Cor. 2-valvis, valv. inferiore herbaceomembranacea oblonga mutica $\nabla$. sub apice setigera. Sar. adnata. (InA. spica.) Spiculae non pedicellatae distichae racheos sulcis, calycis valv. deficientem formantibus, latere dorsali seceptae. 
1. L. perenne: flosc. lancenlatis muticis v. breviter aristatis, spicul. cal. longioribus, culmo glabro, rad. perenni. Mert g. I. 714. S. I. $\delta S$.

a. vulgare. 3. tenue. $\gamma$. compositum. S.l.l. A. $1-2 \cdot 2$. Fl. $5-9$. Undique obvium.

2. L. arvense (Withering): Ilosc. lanceolutis plus minusve aristatis, aristis gluma brevioribus, cal. spiculas subaequante, rad. annua. Mert. g. I. 715. S. I. 39 .

A. 1-1 $\frac{1}{2} ، \odot$. FI. 6, 7. In agris praesertim lino consitis.

3. La temulentum : Hosc. ellipticis aristatis, aristis subrectis valv. longioribus, cal. spicul. aequante, rad. annua. Mert. g. I. 717. S. 1. 89 .

A. $1-3 \cdot \odot$. Fl. 7,8 . In agris praecipue inter segetes copiosum.

$$
\text { 53. MRACHYPODIU II. }
$$

Cal. 2-valv. multiflorus. Cor. 2-valv., valv. inferiore in aristam rectam elongata $v$. subulata, superiore in carina ciliis remotis rigidis setaceis pectinata. Car. subcorticata. (InH. spica dicticha v. spicula terminalis 1 v. 2.) Spiculae breviter pedicellarae.

1. B. sylvaticum (P. Beauy.): spica disticha multiflora muante, spiculis aristatis, aristis flosc. superiorum valv. longioribus, fol. lanceolato-linearibus laxis, rad, fibrosa. Mert. g. I. 6gı. S. I. go.

$$
\text { D } 2
$$


Festuca gracilis Schr.g. I. 343. Bromus gracilis W. A. $1 \frac{1}{2}-2^{\prime} 4 . \mathrm{Fl} .7,8$. In sylvis frondosis. Thierg. Teg. etc.

2. B. pinnatum (P. Beauv.): spica disticha multiHora erecta $\nabla$. subnutante, spiculis aristatis, aristis gluma brevioribus, fol. planis linearibus v. lanceolato.linearibus rigidis, rad. repente. Mert. g. I. 692. S. I. 89 .

Festuca pinnata. Schr. g. I. 342, Bromus pinnatus iW.

a. pubescens. $\beta$. glabrum. S. A. $1 \frac{\mathrm{T}}{2}-2^{\prime} 4$. Fl. 5,6. In collibus et sylvis graminosis spar. sim Teg. Kalkb. Papenb.

54. TRITICUN.

Cal. valv. 2 3-multiflorae ovatae $v$. lanceolatae v. compresso-ventricosae carina distincta. Cor. 2-valv., valv, inferiore actiminata $\nabla$. obtusa $v$. truncata mutica $\mathrm{v}$-carina in aristam desinente, superiore oblonga 2-carinata in carinis tenere ciliata. Car. adnata $\nabla$. libera. (Infl. spica) Spiculae sessiles rhacheos sulcis latere latiore receptae.

* Spiculis ventricosis, vaio. osatis. (Triti. cum Beauv.)

1. T. vulgare (Villars): spica tetragona imbricata, rhachi tenaci, spiculis sub-4-floris, valv, ventricosis ovatis truncatis mucronatis sub apicc com. 
pressis dorsatis carinae nervo obtuse prominente, Gosc. aristatis $\nabla$. muticis, car. liberis. Mert. g. I. 696. a. aestivum: rad. $\odot$. aristis longioribus $T$. aestivum. S. I. 92 . F. hibernum: rad. $\sigma^{\top}$ aristis brevioribus. T. hibernum. S. I.l. A. 3-5' Fl. 6,7 . Planta in agris soli melioris culta.

* Spiculis von ventricosis, valv. lanceolatis $v$. oblongo-lanceolatis. (Agropyrum Beaus.)

2. T. repens: spica disticha, spiculis sub-5-Boris, valv. sublanceolatis 5-nerviis acuminatis, fosc. acuminatis $\mathrm{v}$. obtusiusculis muticis $\mathrm{v}$. aristatis, rhachi plerumque scabra, fol. superne scabriusculis, rad. repente. Mert. g. I. 706 .

Agropyrum repens. S. I. g1。

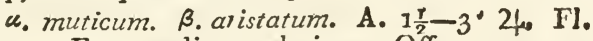
5-9. Fere undique obvium. Off.

3. $T$. caninum: spica disticha, spiculis sub-5-fioris, valv. sublanceolatis $3-5$ nervibus acuminatis, losc. acuminatis, calycib. brevius aristatis, rhachi scabra, fol, utrinque scabris, rad. fibrosa. Mert. g. I. 709 .

Agropyrum caninum. S. I. 91.

Antecedenti similis in nemoribus frondosis passim. Rebentisch. Mundt. S. 1.1.

55. SECALE.

Cal. valy. 2 subulatae 2-llorae c. rudim, losc. 3. 
Cor. valv, 2 valv. inferiore in setam subulata, superiore 2-carinata carinis acutiusculis. (Inf. spica.) Spiculae rhacheos excisuris insidentes.

1. S. cereale: calycibus spicula brevioribus, valv, glabris margine denticulato, rhachi fructifera te. naci. Mert. g. I. 710. S. I. 93.

A. $1-6^{\prime} \sigma^{7}$ et $\odot$, Fl. 6. Planta ubivis culta, 56. ELYMUs.

Cal. valv. 2 anguste lanceolatae $v$. subulatae ante spicul. positae 2-multiflorae, flosc. ఫ్ flosc. superiore saepe imperfecto. Cor. valv. 2 valy. inferiore aristata v. mutica, superiore 2-carinata lineari in carinis tenere ciliata. Car. adnata. (Infl. spica.) Spiculae subternae y. $2-6$ rhacheos excisuris insidentes.

1. E. arenarius: spica crecta contracta, spiculis subtrifloris lanceolatis pubescentibus internediis $8 u b-3$-natis, inferioribus et superioribus geminatis cal. ciliato subbrevioribus, flosc. muticis, fol. involutis rigidis. Mert. g. I. 718. S. I. 93.

A. 2- $4^{\prime} 4 . \mathrm{Fl}, 6,7$. Acervation in arena mobili. Pulyermühlen. Wedding.

Loco spicularum intermediarum ternatarum interdum vidimus geminas.

2. E. europaeus: spica erecta, spiculis sub-2-floris aristatis scabris 3 -natis, cal. Jineasi-subulatis 
aristatis spicularum longitudine, fol. planis glabris, ligulis pilosis. Mert. g. I. 719. S. I. 94.

A. 1-2, 4. Fl. 6, 7. In sylvis frondosis rarius. Jungfh. W.

57. HOR DEUM.

Cal. flosculis antepositus 1-florus 2-valv., valv. lanceolatis v. subulatis. Cor. 2-valvo valv. in. feriore aristata, superiore 2-carinata in carinis dense et tenere ciliata. Flosc. medius $\Varangle$, laterales $\sigma$ v. Rudim. Iosc. superioris setiforme. Car. corticata (Infl. spica.) Spiculae 3.natae.

* Spiculis latera!ibus $\sigma^{\pi}$ et muticis, media ఫ̧ aristata $v$. omnibus $\not{+}$ aristatis et fructiferis. (Hordeum Beauv.)

1. H. vulgare: spiculis omnibus $\Varangle$ aristatis, arista elongata, spiculis 6-fariam positis, seriebus 2 in quovis latere prominentibus. Mert. g. I. 720. S. I. $9^{6}$.

A. $1-2^{\prime} \odot$ Fl. 6, 7. Planta in agris fre. quenter culta.

2. H. hexastichon: spiculis omnibus $\not$, spica elliptica, spicnl. aequaliter 6-fariis. Mert. g. J. 720. S. I. 90 .

Planta $\odot$ praecedenti similis his illisve 10. cis culta. 
* Spiculis lateralibus $\sigma^{7}$. 古, et, sicuti spiculae intermediae, aristatis (Zeocritum. Beauv.)

3. H. murinum: spicularum intermediarum $\nabla$ alv lineari-lanceolatis ciliatis, lateralium non ciliatis setaceis scabris. Mert. g. I. 721. S. I. 64.

frequens.

A. $1-1 \frac{1}{2}^{\prime} \odot$. Fl. $6-8$. In viis, ad sepes etc.

4. H. pratense (Hudson): valv. omnium spicularum et aristis spicularum lateralium calycis valv. brevioribus scabris setaceis. Mert.g.I.722. S.I.95.

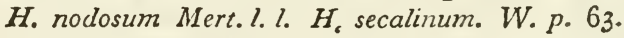

Antecedenti simile, sed majus. 4 Ad pagos, sepes inque pratis passim. W. p. 1.1 .

\section{ORDO III. TRIGYNIA.}

58. MONTIA.

Cal. inferus 2-phyllus. Cor. 1-petala infera 5-fida irregularis. Caps. I-locularis 3-valv.3-sperma. 1. M. fontana: Schr. g. I. 414 . S. I. 96 . Montia minor. L. R. S. II. $87^{\circ}$.

Fol. oblongo-obovata. $\odot$ v. 4 (?). Fl.4-9. Cor. alba. In locis humidis et ad latera lacuum. Templ. Charloub. etc.

59. HOLOSTEUM.

Cal. inferus 5.phyllus, Cor. infera 5-petala pe- 
talis apice serratis. Caps. supera subcylindrica I-locularis valv. 6 apice dehiscens polysperma. 1. H. umbellatum: fol. obiongis glancis glabris, Hor. subumbellatis, caule erecto superne viscosopubescente. S. I. 97 .

A. $2^{\prime \prime}-\frac{1}{2} \odot^{\circ}$. Fl. $4-5$. Cor. alba. In agris et ad vias undique frequens.

\section{TETRANDRIA. \\ O R D O I. MONOG Y N A. 6o. DIPS A CUS.}

Involucr. commune polyphyllum, proprium 1phyllum angulatum. Cal. superus in pappum cyathiformen integrum achaen. coronantem resolubilis. Cor. tubulosa supera 4-fida. Recept. conicum palcaceum. Achaen. 1.

1. D. pilosus: fol. petiolatis appendiculatis, capitulis hemisphaericis, involucris (deflexis) capitulo aequalibus. L. R. S. III. 42.

A. $3-5^{\prime} \sigma^{7}$. F1. 6, 7,8 Cor. albida. Ad sepes et margines sylvarum. Schöneb.

6r. SCABIOSA.

Involucr. commune polyph., proprium 1-phyllum. Recept. paleaceum $\nabla$. pilosum. Cal. superus in 
pappun varie fissum achaen. coronantem abiens. Cor. supera 1-petala 4-5-fida. Achaen.1. * Recept. piloso. (Trichera. Schrader.)

I. $S$. arvensis: caule erecto, fol. ritdicalibus par. tim integris, caulinis pinnatifidis, flor. radiatis, cor. 4-fidis, recept. setoso. H. A. V. .38.

A. $\frac{1}{2}-1 \frac{\pi}{2}{ }^{\prime} 4$. Fl. 6-9. Cor. lilacino-violacea, rarius carnea et alba. In agris sylvisque undique.

* Recept. paleaceo (Asterocephalus. Lagasca. Scabiosa. L. R. S.)

$\div$ Cor. 4-fidis.

2. S. succisa: caule adscendente, fol. radicalibus obovatis v. oblongis utrinque attenuatis, caulinis oblongo-lanceolatis v. lanceolatis utrinque attenuatis, flor. subglobosis. HI. A. V. 37 .

A. $\frac{t}{2}-1 \frac{1}{2} \cdot 2$. Rad. praemorsa. F]. 7, s. Cor. cyauea, lilacina, rarius carnea et alba. In pra. tis sylrisque humidiusculis frequens.

$$
\div+ \text { Cor. 5-fidis. }
$$

3. S. columbaria: cor radiantibus, fol. radicalibus ovatis lyratisve crenatis, caulinis pinnatis pinnis incisis, calyce involucro multo longiore. S. I. 100 .

A. $\frac{r}{2}-1^{\prime}$ 24. Cor. coeruleo-lilacina. Fl. 6,7• In collibus et agris siccioribus Papenb. Kalkberge etc. 


\section{SCIIEARกIA.}

Cal. superus margo 4-dentatus. Cor. 1-petala, in. fundibuliformis. Achaen, 2 cal, persistente coronata 3 -dentata.

1. S. arvensis: caule scabro, fol. inaequalibus inferioribus 4-8, flor, terminalibus. S. I. 101.

A. 2-6" ค. Tl. 7,8. Cor roseo-lilacina. In agris argillosis. Schöneb. Rüdersd.

\section{A SPERUIA.}

Ca?. margo obsoletus 4-dentatus. Cor. tubulosoinfundibuliformis. Achaen. 2 glubosa calyce libera.

1. A. odorata: fol. 8 -nis lanceolatis, corymbis terminalibus pedunculatis, achaen. echinato-hispidis. I. R. S. III. 265.

A. $\frac{r}{2}-2^{\prime} 24$. Rad. repens. Fl. Cor. Colba. In sylvis nmbrosis sublumentibus. Papenb. Brisclang.

2. A. tinctoria: fol. linearibus, inferioribus senis 3-nerviis, intermediis 4-ternis, sumnis oppositis, caule flaccido, cor. glabris 3-fidis. L R.S. III. 268 . A. $\frac{r}{2}-2^{\prime} 24$. Rad.repens. Fl. 5, 6. Cor. rubicunda. In collibus graminosis. Papenb. Kalk. berge.

3. A. cynanchica: fol. inferioribus 4-nis lanceolatis, supericribus linearibus maxime inaequalibus, binisve, internodiis brevioribus, caule erecto, cor. 
4. fidis, pedunculis trichotomis, achaen. subtuberculatis. L. R S. III 269 :

A. $\frac{r}{2}-2^{\prime} 24$. Rad. fusiformis. F1. 5, 6, 7. Cor. albo-rabicunda. In apricis sylvarum. Spand. Grunw. etc.

$$
\text { 6. GAIIUM. }
$$

Cal. margo obsoletus superus $\nabla$. minimus 4-5dentatus. Cor rotata. Achaen. 2.globosa cal. vacua.

* Achaeniis glabris laevibusque.

1. G. sylvaticum: fol. 8-nis lanceolatis margine carinaque scabris, pan. terminali diffusa, caule toreti superne glabro. S. I. 102.

A. $\frac{\mathrm{r}}{2}-2^{\prime}$ 24. Fl. 6, 7. Cor. minutissima, alba. In sylvis frondosis. Briselang. S.

2. G. verum: fol. 8-nis linearibus sulcatis scabris, Hor, paniculatis congestis, rad. repente. S.I. 103.

A. $1-2,24$. Fl, 6, 7, 8. Cor. lutea. In agxis siccioribus nbivis obvia.

3. G. Mollugo: fol. 8-nis elliptico-lanceolatis mucronatis, margine scabris, flor. paniculatis divaricatis, rad. repente. S. I. 103.

๙. glabratum. $\beta$. pubescens. A. anteced. 24. FI. 7,8 : Cor. albo-lutescens. In campis ubi. vis obvia.

4. G. palustre: fol. 4-ternis obovato-lanceolatis 
obtusis inaequalibus caulibus diffusis sublaevibus, flor. paniculatis divaricatis, achaen. cor, aequantibus. S. 1. 104.

A. 4-8" 4. Fl. 5, 6. Cor. alba. In locis udis ubique.

* Achaen. tuberculatis glabris.

1. G. saxatile: fol. 4-ternis senis obovato-lanceolatis mucronatis scabris, caulibus procumbentibus laevibus, flor. paniculatis arctis, achaen. cor. brevioribus. S. I. 104 .

G. harcynicum. var. Auctor.

A. 4-6" 4. FI. 5, 6, 7. Cor. alba antheris flavescentibus. In sylvarum muscosis locis. Papenb. Grunw. Cöpn.Heide.

6. G. uliginosum: fol.6-8-nis lanceolatis mucronatis margine scabris, caulibus flaccidis retrorsum scabris, for. divaricato-paniculatis, cor, achaeniis majoribus. S. I. 104.

A. $\frac{\mathrm{r}}{2}-1 / 24$. Fl. 6,7, s. Cor. alba. In locis uliginosis frequens.

7. G. spurium: fol. 6-8-nis oblongo-lanceolatis mucronatis carinatis, carina margineque scabris, caule adscendente scabro, pedunculis simplicibus subtrifidisve, cor. achaeniis minoribus, S. I. 105.

A. $\frac{r}{2}-1$ ' $\odot$. Fl. 5, 6, 7. Cor. albida. In agris fertilioribus cultis، Cöpn. Feld, Hasenlieide. $S$. 


$$
\text { * Achaen. hispidis. }
$$

8. G. Aparine: fol. 8-nis oblongo-lanceolatis mucronatis carina margineque retrorsum aculeatis, caule flaccido hirto, geniculis villosis, cor. achaeniis pilis uncinatis tectis minoribus. S. I, 105.

A. 2-6' $\odot$. Fl. 5-8. Cor. parva, albida. In locis humidis inter frutices frequentissimum.

9. G. boreale: fol. 4-ternis lanceolatis aut ovalilanceolatis 3 -nerviis margine carinaque antrorsum scabris, caule erecto, corollis achaen. pilis uncinatis hispidis majoribus. S. I. 106.

A. $\frac{r}{2}-3 \cdot 2$. Rad. repens. Cor. alba. In pratis et sylvis frequens. Friedrf. Papeub, etc.

$$
\text { 65. CORNUS. }
$$

Cal. superus 4-dentatus. Cor.4-petala. Nect. glandula styli basin cingens. Drupa nuce 2-loculari.

* Umbellis involucratis.

1. C. mascula: arborea, umbellis involucri magnitudine. L. R. S. III. 318 .

A. $18-20^{\prime}$. F Fl. 4. Cor. Inteae foliis prio. res. In sepibus et nemoribus hinc inde. Thitrgart. Teg.

* * Cymis nudis.

2. C. sanguinea: ramis rectis glabris v. subverrucosis, fol. oppositis ovato-ovalibus acurninatis subtus pallidioribus subpubescentibus, cymis de- 
pressis, dentibus cal. nectarii longitudine, drupa globosa nigra, nuces globosae. H. F. 7 •

A. $10-12^{\prime}$ 万. Fl. 6. Cor alba nect. flavo. In sylvis et pratis hinc inde. Thierg. Teg. etc.

3. C. alba: ramis recurvatis verrucosis, fol. ovalibus oppositis longe acuminatis subtus canis sub. pubescentibus, cymis depressis, dentibus calycis nect. longitudine, drupa globosa alba, nucibus compressis. H. F. 7 .

A. 6-8' 万. Fl. 6. Cor. alba, nect. rubro. Hinc inde culta. Thierg.

66. TPAPA.

Cal. superus 4 -partitus. Cor a-petala supera. Nux spinis $2-4$ (e calycis foliis natis) oppositis cincta 1-locularis 1-sperma,

1. T. natans: nucibus plerumque 4-spinosis, spinis patentibus. S. I. 107.

A. varia. $\odot$. Fol. submersa capillacea, natantia rhomboidea, dentata. F1.5, 6. Car. alba. In lacubus. Müggelsee.

Variat, nt et Mert. g. I. \$22. monet, nucibus 2-spinosis, quam varietatem quidem nondum apud nos, satis frequentem autem Vitebergae, observavimus.

$$
\text { 67. II A N TAOO. }
$$

Cal. inferus 4-sepalus. Cor, infera hypocrateıformis limbo 4-partito repando. Stam. longis- 
sima. Caps. circumscissa spermophoro libero dissepimentiformi quasi 2 -locularis 2-poly. sperma.

* Caule nudo (scapo). (Plantago.)

1. P.major: fol. longe petiolatis ovatis glabrius. culis petiolo brevioribus, scapo tereti, spica cylindrica gracili, floribus arcte imbricatis, caps. polyspermis. L. R. S. III. 111. H. A. V. 13.

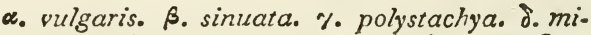
crostachya. H. l. l. A. $\frac{\mathrm{r}}{8}-1 \frac{1}{2}{ }^{\prime} 4$. FI. 5-9. Cor. albo-brunescens. Planta variabilis ubivis obvis.

2. P. media: fol. ovalibus pubescentibus petiolo Iongioribus, scapo tereti, spica brevi cylindrica, caps. 2-spermis. H. A. V. 14.

«. major. B. minor. H. l.l. A. $\frac{\mathrm{r}}{8}-1^{\prime}$ Fl. $5-$ B. Cor. alba filamentis lilacinis. In agris graminosis copiosissima.

3. P. lanceolata: fol. breviter petiolatis lanceola. tis subpubescentibus, pedunculis angulatis, spica conico-ovata 'glabra, caps. dispermis. S. I. 109. I. A. V. 15.

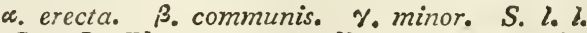
A. $\frac{\pi}{8}-1 \frac{\pi}{2}$. Fl. 5-g. Cor, alba nervis bruneis. Ubivis obvia.

\section{* Caule ramoso (Psyllium).}

5. $P$. arenaria: subviscido-pilosa, caule herbaceo ranoso, fol. linearibus planis subintegerrimis, ca- 
pitulis involucratis, sepalis heteromorphis. H. A. V.16. S. I. 110.

A. $\frac{1}{4}-1^{\prime} \odot$. Fl. 6-s. Cor. brunescens tubo inflata. In campis arenosis frequens. Charlottb. Spand. etc.

68. CENTUNCUIUS.

Cal. 4-fidus inferus. Cor. 4-fida infera tubulosa

limbo patente. Stam. brevia. Caps.1-locularis

circumscissa polysperma spermophoro centrali.

I. C. minimus: fol. alternis ovatis, flor. sessilibus. L. R. S. III. 110.

A. 1-3" $\odot$. F1. 6. 7: Cor. rubicunda. In locis argillosis humidis sparsim. Weilss Grunw.

69. SA N C U X SORBA.

Cal. 4-sepalus foliol. receptaculi collo insertis perpersistentibus Cor. o. Germ. recept. insertum. Achaen, recept. inclusum.

3. S. officinalis: spicis oblongis subrotundisque, stam. calyce brevioribus, cal. foliisque glabris. S. I. 111. HI, A. VIJI, 22.

A. 2-3.24. Fl. 6, 7. Cal sanguineus. In locis humidis graminosis satis frequens Thierg. Papenb. etc.

70. ALCHEMILIA.

Cal. 8-fidus laciniatus laciniis alternis minoriF. B.

E 
bus Cor. o. Styl. e basi germinis Achaen. I-2 calyce vestita.

I. A. vulgaris: fol. reniformibns plicato-concavis serratis, caule petiolisque glabriusculis, Hor. dichotome-corymbosis L. R. S. III. 468 .

A. $\frac{1}{2}-1$ ' 24. F1. 5, 6. Cal. viridescenti.lutens. In collibus et pratis humentibus. Schönh. W. Heiligens. S. Tafsd.

2. A. Aphanes: fol. 3-partitis laciniis $2-4$ partitis pubescentibus, flor. axillaribus glomeratis 1 -an. dris. S. I. 112 .

A. $2-6$ $\odot$. Fl. 7, 8 In agris argillosis sparsim frequens. Panck. Weilss. etc.

$$
\text { 71. IARIETAPA. }
$$

Poiggama. Cal. 4-fidus inferus. Cor. o. Achaen. 1 superum elongatum cal. persistente inclu. sumı.

1. P. officinalis: fol, ovatis acuminatis basi cnneiformibus scabriusculis, glomerulis amplexicaulibus, pedunculis dichotomis. H. A. V. I2.

A. $1-2,2$ 4. Fl. 7, S. Flor. 호 et 오. Ad muros ruderata et ad sepes rarius. Schöneb.

\section{ORDO II. TETRAGYNIA.}

72. POTAMOGETON.

Cal. o. Cor. infera 4-partita Germ. 4. stylis o. stigm, sessili. Achaen. 4. dupracea sessilia. 
- Fo? natantibus et linearilus n.

1. P. crispus: caule compressiusculo, fol. lanceo latis serratis undulatis S. I. I13.

A. varia $24 . \mathrm{FI}$.5.6. Satis frequens in aquis. stagnantibus et lenitor fuentibus. Thierg. Grunw. etc.

2. P. perfoliatus: caule tereti, ful. cordatis amplexicaulibus obtusis. S. I. $1: 3$.

A. varia $4 . \mathrm{Fl} .6,7$. In lacubns et Huviis. frequens.

3. P. praelongus (TWulffen): canle tereti flexuoso, fol. semiamplexicaulibus orato.oblongis apico raviculatis, pedunculis aequalibus S. I. 113.

A. varia 24. Fl. 5, 6. Porius in Auminibus et lacubus. Teg. Grunw.

4. P. lucens: caule tereti, fol. acuminatis basi attenuatis sessilibus aut superioribus brevissima petiolatis, pedunculis incrassatis. S. I. 114.

a. vulgaris. 6. nitens. A. raria. 24. Fl. $5,6$. In huviis et lacubus frequens Teg. IVeifss. * Fol. superiorious natantibus nec linea. ribus.

5. P. rufescens (Schrader): canle tereti, fol. ob. tusis, inferioribus lanceolatis trinerviis sessilibas, superioribus obovatis in petiolo folio breviorem attenuatis, pedunculis aequalibus. S. I. 114.

A. varia. 24. Fl. 5.6. In fossis es rivalis. Willmerd. S. Zwischen Stadt. 11. Jungfh. etce E 2 
6. P. natans: canle tereti, fol. omnibus petiolntis acuminatis natantibus aut fluitantibus pedunculis aequalibns S. I. 115.

ж. vulgaris ( $P$. natans $K$ th. 48.) B. fluviatilis (P. fluitans Kth. 49) A. varia 24. Fl. 5, 6. In aquis undique frequens.

7. $\boldsymbol{P}$. heterophyllus (Schreber): caule tereti flexuoso, fol, submersis sessilibus recurvatis lanceolatis aculis, summis in natantia evadere conantibus pedunculis incrassatis S. I. 115 .

P. gramineum Kth. 48. (?)

a. palustris. B. fluviatilis. \% lacustris. $\delta$. terrestris. A. varia. 24 . Fl. 5,6 . In stagnis $\alpha$, fllviis $\beta$, lacubus $\%$, in terra humida $\delta$, obvia $S$. * * Fol. omnibus linearibus nec vaginantibus, natantibus o.

8. P. Zosterifolius (Schumacher): caule complanato, fol linearibus basi eglandulosis. S. I. 116.

P. complanatum. Kth. 50.

A. varia. 4 . Fl. 5, 6. In aquis profundioribus passin. Grunw. Teg.

9. $P$. compressus: caule complanato, fol. linearibus basi biglandulosis, achaen, rotundatis compressis carinatis. S. I. 117.

*. obtusifolius. $\beta$. acutifolius. A. varia. 2 .

Fl. 6, 7. In lacubus fossisque frequens.

to. P. pusillus: caule compressiusculo tereti, fol. 
linearibus basi biglandolosis, achaen, oblongoovatis compressiusculis ecarinatis S. I. 117•

A. varia. 24. Fl. 5, 6 Hinc inde in stagnis fossis et lacubus. Teirss. Spand. etc.

* * Fol. omnibus linearibus vaginantibus, natantibus 0.

11. P. pectinatus: caule tereti, fol. linearibus basi vaginantibus, spica interrupta. S. I. 117.

$\alpha$. stagninus, $\beta$. lacustris. A. varia, 24. Fl. 5, 6. Rarius in locis arenosis aquarum. Teg. S.

$$
\text { 73. } S \Lambda \mathrm{GINA} \text {. }
$$

Cal. inferus 4-sepalus. Cor. 4-petala. Germ. su. perum, styiis 4. Caps $1-$ locularis $4-\nabla a l v$, polysperma spermophoro centrali columnari.

1. $S$. procumbens: ramis procumbentibus glabris, petalis brevissimis, sepalis obtusis. S. I. 118. A. 2-4" $\odot$. Fol. subulata. Fl 5-7. Cor. alba, interdum $o$. In terra humida undique frequens.

\section{1. V. PEN T A N D R I . \\ ORDO I. MONOGYNIA. \\ 74. MYOSOTIs.}

Cor. hypocrateriform. fauce squamis brevib. instructa, Stam. inclusa. Achaen 4. fundo calycis aflixa basi umbilicata brevissime marginata. 
* Calycis basi pilis rectis instructa.

1. M. palustris (Withering): perennis, patentin pijosa, racem. adpresso-strigoso-pilosis, cal. 5-dentatis, dentib. orat. acutiusculis, stylo calycen aequante aut superante, achaen. laevib. glabris. S. I.11g. Myosotis scorpioides. W. et $K t h$. (ex parte).

A. $\frac{1}{2}-2,2$. Fol. lanceolata. Fl. 5, 6, 7. Cor. coerulea. In aquis et paludibus undique conspicua.

2. M. cespitosa (Schultz): annua, tota adpressostrigoso-pilosa, cal. 5-partitis, laciniis lanceolat. acutiusculis, stylo cal. dimidio breviore, achacn. laevib. glabris. S. I. $11 \mathrm{~g}$.

Myosotis scorpioiles. W. et Eth. (ex parte).

A. $\frac{I}{2}-1$ varia. $\odot$. Fol. lanceolata. Fl. $5,6,7$. Cor. pallide coerulea. In his illisve locis hu. midis frequens. Spand. Temph. S. Neifs.

$$
\text { * * Calycis basi pilis uncinatis instructa. }
$$

3. $M$. intermedia (Link): biennis, caule patentim hirsuto, pedunculis cal. fructifero clauso duplo Iongioribus, stylo cal. duplo breviore, corollac limbo infundibuliformi. cal breviore. S. I. 120. Myosotis arvensis. W. et Kth. (ex parte).

a. agrestis $\beta$. sylvestris. A. $\frac{1}{2}-2^{\prime}$, ${ }^{\star}$. Fol. ovato-lanceolata Fl. 5,6 . Cor. pallide coerulea. In agris et sylvis. Temph. Thierg. etc. 4. M. versicolor (Roth): annua, caule patentim 
liirsuto, peduncul. cal. patente brevioribus, stylo calycem subaequante, corollae limbo erecto cal. duplo breviore. S. J. 120.

Myosotis arvensis IT. et Kt/l. (ex parte).

A. $\frac{1}{7}-1^{\prime}$ ○. Fl. 5, 6. Cor. primunı lutea dein violascenti-cocrulea. In agris et locis humidiusculis. Spand. Templ. Grunw. etc.

5. MI. hispida (Schlecht.ndal): annua, caule patentim hirsuto, peduncul calycem fínctifer. patent. aequantibus, stylo et corollae limbo erecto cal. 4.plo brevioribus. S. I. 121.

Myosotis artensis $W$. et $K$ th (ex parte).

A. $\frac{1}{4}-\frac{3}{4}$ 'Fol. ovato-lanceolata $\odot$. Fl. 5-8. Cor, coerulescens. In agris et sylvis subhumidis copiosa. Grunw. Templ. elc.

6. M. arenaria (Schrader): annua, caule patentim birsuto, peduncul. cal. fructifero clanso brevioribus, stylo et corollae limbo erecto cal, 4-plo breviore. S. I. 121.

Myosotis stricta Link. Myosotis arenaria W. et Kith. (ex parte).

A. $\frac{1}{8}-\frac{1}{4} \cdot \odot$. Fol. orato.lanceoIata Fl. 5,6,7. Cor. coerulescens. In campis et sylvis frequentissina.

\section{I A P X U. A.}

Cor. hypocrateriform. fauce squan, brevib. instructa. Achaen. 4. columnae centrali affixe echinata compressa basi non umbilicata. 
1. L. Myosotis (Moench): caule superne ramoso, fol. lanceol. incumbenti-pilosis ciliatis, cor. parva limbo erecto-patente, achaen. aculeis glochidiatis duplici serie margine dispositis. Lehm. A. I. 121. Myosotis Lappula. Echinospermum. L. Lehm. l. l.

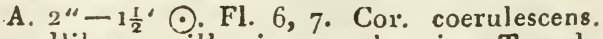
In collibus argillaceis. v. calcareis. Temph. S. Ruedersd.

\section{CYNOGLOSS UMr.}

Cor. infundibuliform. fauce fornicib. erect. instructa. Achaen. 4-depressa echinata v. margine membrana plana circumdata columnae centrali affixa basi ir, erforata.

1. C. officinale: caule erecto folioso, fol. inferiorib. lato-lanceol. basi attenuatis, superiorib. basi latiorib. acut. pubescenti-tomentosis, racem. ebracteat. durante florescentia paniculatis, stam. inclu. sis. Lehm. A. 152. HI. A. I. 26.

A. $1-3^{\prime} \sigma^{\top}$. Fl. 5-7. Cor. purpurea. Ad pagos, in ruderaits etc.

77. ASPERUCO.

Cor. subinfundibuliform. fauce squamis clausa. Achaen. \& compressa columnae centrali affixa basi integra.

1. A. procumbens: cal. fructiferis compressis. L. W. I. 778 . 
A. $\frac{\mathrm{r}}{2}-1 \frac{\mathrm{T}}{2}{ }^{\prime}$ - . Fl. 5, 6. Fol. oblonga, scabra. Cor. purpureo-violacea Ad vias et in ru. deratis. Thierg, Panck. etc.

78. A N с HUS A.

Cor. infundibuliform. fauce fornicib, erect. ob. tusis clausa. Achaen. 4. rugosa turbinata fundo calycis affixa basi concava umbilicata.

1. A. arvensis (Bieberstein): caule erecto ramoso, fol. lanceol. semiamplexicaul. subrepando-denticu. lat. strigoso-hispidissimis, racem. foliosis, laciniis calycis longitudine tubi incurvi, corollae inaequalis laciniis lanceolatis. Lehm. A. 226. S. I. 124. Lycopsis arvensis W. et Kil

A. 1-2' $\odot$. Fl. 5-8. Cor. coerulea. In agris undique.

2. A. officinalis: fol. lanceol. hispidis, bracteis orato-lanceolat. calycem aequantibus cal. 5-fid. acutis. Lehm. A 246. S. I. 12 f.

Anchusa angustifolia Kith. 5 i.

A. $\frac{1}{2}-2^{\prime} 24$. Fl. $5-7$. Cor. violacea v.coe. rulea, rarius alba. In agris ad vias, in coeme. teriis, circum pagos. etc.

\section{IUIMONARIA.}

Cal. 5-fid. prismatico 5-agonus, in fructu cam. panulatus. Cor. infundibuliform fauce pervia pilosa. Achaen. 4. turbinata fundo calycis affixa basi integra. 
1. P. ofjicinalis: fol. scabris radicalib. orato-cor. dat. petiolatis, caulinis superiorib. sessilib. ovato. oblongis. Lehm. A. 277. H. A. II. 4氏.

A. $\frac{x}{2}-1^{\prime}$ 4. Fl. 4. Cor. coeruleo-purpurascens. Fol. maculata. In sylvis frondosis. Briselang.

SO. IITHOSPEPITUM.

Cal. 5-partitns. Cor. infundibuliform. fance pervia nuda. Achaen. 4. ovata findo calycis aftixa basi imperforata.

1. L. officinale: caule herbacco tcreti erecto ramosissimo, fol. lato-lanceolat. acut. venos, scaber: rimis, corollae tubo longitudine calycis. Lehm. A. 310. If. A. VI. 29.

A. 1-2, 24. F1. 5, 6. Cor. albido-flavescens. Achaen. laevia. In collibus argillaceis v. calcareis inter frutices. Puzedersd.

2. L. arvense: caulib. herbaceis erect. ramosis, fol. lanceol. basi angustat. acutiusc. hirt. subciliatis, cal. corolla paullo breviorib. in fructu patu. lis, achaen. rugos. scabris, Lehm. A. 320.

A. $\frac{1}{2}-2^{\circ} \odot$. Fl. 5-6. Cor. alba aurulo violaceo Achaen. tuberculosa. In agris undi. que frequens.

S1. SYMIITUM.

Cal. 5-partitus, Cor. cylindrico-campanulata fauce fornicib. 5. subulat. in conum conniren. 
tib. instructa. Achaen. 4. ovata fundo calycis affixa basi non perforata.

1. S. officinale: caule ramoso superne alato, fol. ovato-lanceol. basi attemut. decurrentib. supra scaberrim. subtus hirsutis, cal. acut. hirtis. Lehm. A. 351 . H. A. III. 37.

A. $1-3,24$. Rad. subcylindrica, Fl. 5, 6 . Cor. alba $r$. violacea. In humidis et paludosis locis.

82. ECIIIUTr.

Cal. 5-partitus, Cor. infundibuliform. v. campr. nulata fauce ampliata nuda, limbi laciniis saepius inaequalibus. Achaen. 4. turbinata gi. gartoidea scabra fundo calycis affixa basi im. perforata.

1. E. vulgare: caule herbaceo subsimplici tuberculato- lispido, fol. lineari-lanceol. hispidis, racemo terminali spiciformi longissimo, spic. singul. lateral. simplicib. patentibus, stam. corolliz irregulari longioribus. Lehm. A. 449. H. A. I. 27. S。 I. 226 .

A. $\frac{r}{2}-2^{\prime} \sigma^{7}$. Fl. 5-7: Cor, coeruleo-violascens. In agris siccioribus, muris etc. frequens.

$$
\text { 83. ANAGALIIS. }
$$

Cal. 5-partitus Cor. rotata, Caps.1-locul, poly. sperma sphermoph. pedicellato globoso centrali. 
1. A. phoenicea (Lamark): caule prostrato, fol. ovatis, laciniis calycis integerrim. corollae margine glandulifero brevioribus. S. I. 127.

Anagallis arvensis, H. A. II. 46.

A. $\frac{1}{4}-\frac{3}{4}$ 'Fl. 5-8. Cor. miniata. In agris et ad vias. frequens.

2. A. coerulea: canle suberecto, fol, oblongo-ovatis, laciniis calycis serrulatis, corollam eglandulosam aequantibus, H. A.II. 46 . S. I 127.

A. $\frac{r}{4}-\frac{1}{2}^{\prime} \odot$. Fl. 5, 6, 7. Cor. coerulea. In aoris rarius ante portam Halensem et prope Schoeneb. IV'. S.1.1.

\section{PRIMULA.}

Cal. 5-dentatus. Cor. hypocrateriform. tubo cylindrico. Caps. 3.locul. polysperma apice 10. dentata dehiscens spermophoro centrali subclavato.

1. P. veris: fol. dentatis rugosis subtus hírsutic, umbella multiflora, flor. omnib. nutantibus, cal. angulatis, corollae limbo concavo brevissimo. Lelim. Prim. 27. H. A. III. 34. S. I. 127.

A. $\frac{1}{2}-1$ 24. F1, 4, 5. Corolla flava. In sylvarum frondosar. collibus herbosis his illisve copiose. Panck. Papenb. Kalkb. Friedrf. etc.

85. HотTONIA.

Cal. 5-partitus, Cor, hypocrateriform. Caps, 1-10- 
cul. polysperma evalv. spermoph. centrali pedicellato globoso.

1. H. palustris: flor verticillatis pedinculatis, fol. omnib. immersis. L. R.S. IV. 176 . S. I. 128.

A. varia 4. Fl. 5. Rad. repens. Fol. pinnatifida. Cor. pallide rosea In paludibus fossisque frequentissima.

86. X I SIMACHIA.

Cal. 5-partitus. Cor. rotata. Stam. basi dilatata v. connata. Caps. 5-10-valv. 1-locul. polysper. ma spermoph. centrali globoso.

* Caule erecto.

1. L. vulgaris: fol. oppositis 3 r. 4 oblongo $\nabla$. lanceolato-ovalibus, racemis terminalibus, superiore composito, inferioribus simplicibus, stam. cor. brevioribus. H. A. VIII. 15. S.I. 129.

A. $1-42 \%$ R.zd. sobolifera. Fl. 6,7. Cor. lutea. In pratis, ad fossas et inter frutices frequens.

2. L, thyrsiflora: fol. oppositis lanceolatis, racemis axillaribus pedunculatis conglomeratis, stam. cor. longioribus. S. I. 129 .

A. $\frac{T}{4}-1 \frac{T^{\prime}}{2} 4$. Fad. repens. $T l .5$. Cor. lutea. In locis paludosis frequens, Charlottb. Grunw. Thierg. etc.

* Caule repente.

3. L. Nummularia: caule repente glabro, fol. sub- 
cordatis oppositis glabris, pedunculis axillaribus solitariis 1-foris. II. A. VIII. 16. S. I. 130.

Fl. 6 - - 2 f. Cor. lutea. In solo humido fextili undique.

87. MENYANTHES.

Cal. 5 - partitus. Cor. infundibiliform, intus villosa. Stigm. 2-lobum. Caps. 1-locul. 2-valv. v. cralv. polysperma spermoph. 2 opposit. linearib. parietalibus.

3. M. trifoliata: fol. ternatis. H. A. III. 14. S. I. 130.

A. $\frac{r}{2}-1^{\prime} \psi$ Caulis (non rad.) repens. Fl. 5. Cor. alba. In aquis et paludibus frequens. Ớf. SS. ERTIIRAEA.

Cal 5-dentatus. Cor, infundibiliformis. Antherae defloratae spirales. Caps. 2-valv. polysperma marginibus utriusque valv. intus flexis mar. gineque involuto seminiferis quasi 2-locularis.

1. E. Centaurium (Persoon): caule 4 -gono integro, fol. oblong. acut. 3-5-nerviis, corymbo fasciculato multifloro.

Gentiana Centaurium L. Chironia Cent. H. A.I. 29. a. mlgaris s. corymbosa. B. capitata. (E. capitata Halb. W.) S.I. 130. A. $3^{\prime \prime}-1^{\prime} \odot$. Fl. 6,7 . Cor. roseo-lilacina. In pratis satis frequens. Charlotib. Spand. etc. Off. 
2. E. Linarifolia (Persoon): canle 4-gono inaequilatero compresso, fol. lanceolato-linearib. obtusis subuninerviis, corymbo fasciculato-dichotomo paucifloro, flor, terminalibus alaribusque. Reichenb. Icones plant. rav. Cent. I. Dec. IX. LXXXVIII.

Erythraea compressa. Hayne Kith.65. S. I. 131.

Antecedenti simillima iisdemque locis, sed rarius, obvia. Zwischen d. Fasancrie a. Will. mersd. Kth. IVeilss.

2. E. inaperta: caule 4.gono deliquescente dichotomo-corymboso, fol. oblong. obtus. obsolete 3 . nerv. integerrimis, Hor, terminalibus alaribusque. Kth. 65 .

Chironia inaperta. II. A.I. 30.

A. $2-4 " \odot$. Fl. 6, 7. Cor. roseo-lilacina. In pratis minus graminosis et ad latera la. cuum. Weifss. Spand. etc.

S9. VIOIA.

Cal. 5-sepalus $\nabla$. 5-partitus, Cor. 5-petala irregu. laris corniculata. Anther. coalitae apice membranaceae. Caps. 3.valv polysperma spermoph. 3 medivalvibus.

\section{* Acaules.}

1. $V$. hirta: acaulis villoso-hirta, fol, suboratocordatis, stipul. lanccol. acuminat. integerrimis, sepal. ovat. obtusis, petal. emarginatis, caps. subglobosa. S. I. 132. H. A. III. 1. 
A. $2-3$ "24. Fl. 4, 5. Cor. pallide violacea. In collibus et inter frutices. Papenb. Kalkb S. Grunw.

2. V.odorata: acaulis stolonifera pubescens, fol. subrotundo-cordatis, stipul. lanceol. acuminat. sparsim setaceo-serratis, sepal. ovat. acutiusculis, petal. integerrimis, caps. subglobosa. S. I. 132. H. A. III. 2 .

A. 2-3" 24. Fl. 3, 4. Cor. laete violacea. In graminosis rarius. Temph.Berg. S. Schönh. Thierg. Off.

3. V.palustris: acaulis stolonifera glabra, fol. rotundato-reniformibus, stipul. lato-lanceol. subserratis, sepal. ovat. obtusis, petal. integerrimis, caps. cylindrica obtusa. S. I. 133

A. $2-3$ " 24. Fl. 4,5 . Cor. pallide coerulea. In sylvis liumidis copiosa. Thierg. Grunw. etc. * Caulescentes.

3. I. canina: caule pubescente plerumque ad. scendente, fol. ovat. cordais, stipulis lanceol. acuminat. setoso-serratis, sepal. lanceol. acuminatis, petal. subintegerrimis, capsula oblonga. S. I. 133. II. A. III. 3 .

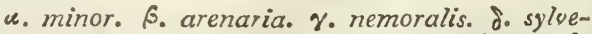
stris. S. l.l. A. 2-6"24. Fl. 4-5. Cor. pal. lide violacea. In sylvis collibus et pascuis frequens.

4. V. tricolor: caule angulato glabro, fol, crena- 
to-serrat. subglabris, inferioribus ovato oblongis, superioribus oblongo-lanceolatis, stipul.lyrato-pinnatifidis, sepal. lanceol. acuminatis, petal. integer. rimis, caps. oblonga caly cem subaequante. S I. 135. H. A. III. 4.5 .

a. pariflora. B. grandiflora H. l.l. A. $\frac{\bar{\pi}}{4}-\mathbf{1}^{\prime}$ $\odot$ et $\sigma$ (non 24). FI. 4-10. Cor. alba et lutea vo alba lutea et villacea v. violacea et lu. tea v. violacea. In solo sicciore frequens. Off.

$$
\text { 90. IMPATIENS. }
$$

Cal. disepalus deciduns. Cor. 5-petala irregularis petalo superiore sub-3-fido, '2 inferiorib. maximis. Anther apice connatac. Caps.polysperma 1-locul. 5-valvis, valv. elastice desilientibus eoque spiram formantibus. spermoph. columnari.

1. I. Noli tangere: peduncul. multiflor. solitariis, fol- ovatis serrati, geniculis caulinis tumentibus. L. R. S. V. 350 . S. 1. 135.

A. 2-3' $\odot$. Fl. 7, 8. Cor. Intea. In sylvis humidis sparsim. Friedrf. Clamisso Brise. lang S. Gruuw.

91. EV O NYMU S.

Cal. 5-partitus. Cor. 5-petala calyci inserta. Caps. 5-gona 5-locul. 5-valv. colorata, locul. 1-spermis. Sem, arillata. (Partium numerus inter. dum quaternarius.)

F. B. 
1. E. europaea: ram. glabris, fol. oblongo.lanceol. serrat glabris, peduncul. compresso-filiformib. 3-foris, flor. 4-andris, caps. 4-gon. glabris, angulis subacutis. H. F 23.

A. 6-20' T. $\nabla$. 万. Fl. 5. Cor. luteo-alba. Fruct. purpurei. In solo fertiliori, in sylvis, ad sepes. Thierg. Panck. etc.

$$
\text { 92. VFR BA SCUM. }
$$

Cal. 5-partitus. Cor. rotata. Filcm. distantia bar. bata. Caps. 2-locul. 2-valv. (valv. saepe 2-fidis) polysperma, dissepimento ex inflexis marginibus formato.

* Foliis decitrentibus.

i. V. Thapsiforme (Schrader): fol. decurrentibus crenulatis tomentosis, superiorib. acuminatis, racemo spicato denso, cor. rotatae laciniis obovatorotundatis, anther. 2 oblongis. Schrader Verb. I. 12 .

I'erbascum Thapsus Kth. 62.

A. $\frac{x}{2}-2^{\prime} \delta^{7}$. Fl. 6,7 . Cor. Iutca. In agrik arenosis. Off.

$$
\text { * Foliis petiolatis. }
$$

2. V. Iychnitis: fol. crenat. supra glabriusculis subtus pulverulento-tomentosis, inferiorib. ovalioblong. obtusiuscul. in petiol. attenuatis, superio. rib. ovato-oblong. acut, subsessilibus, summ. ovar. 
scuminatis, racem. paniculatis, pedicell calyce duplo longioribus. Schrader Verb. IJ. 1 S.

A. $1 \frac{r}{2}-4^{\prime} \sigma^{\nearrow}$. Fl, $;-8$. Cor. lutea, Filamentorum lana albida. In agris coemeteriis ad vias etc. frequens.

3. $\%$ nigrum: fol, crenatis supra glabriusc, subtus tenuissime tomentosis, inferioribus et caulin. oblongo-ovat. cordat, petiolatis, superiorib. ovato. oblong. subsessilibus, racemo elongato, pedicellis cal, duplo longioribus, Schrader. Verb.II, 2 q.

A. $1 \frac{1}{2}-3 \cdot 4$. Fl.5-8. Cor. lutea, Filamen. torum lana purpurea. Fere undique obvium.

93. CONVOLVULUS.

Cal. 5-partitus, Cor. campanulata plicata, Caps. 2-3-valv. 2-3-locul. locul, 2-spermis, disse. piment. valvaribus.

1. C. arvensis: caule rolubili prostrato fol. se. gittat. utringue acutis, peduncul. teretib, subuniflor. axillaribus, bracteis linearib. remotis, laciniis calycis obtusis, S. I. 137.

Fad. repens, 24. FI. 6-9. Cor, albido-rosea v. rosea In agris undique conspicuus.

2. C. sepium: caule volubili, fol. cordato-hastat, postice truncatis, peduncul. sub-4-gon. 1-Hor. axillaribus, bracteis cordat, calyces includentibus; $l_{2}$. ciniis cal. acutiusculis, S. I, 137. 
Calystegia sepium. L. R. S. IV. $1 \delta_{2}$.

Rad. repens. Fl. $6-9$ Cor. magna nivea. In solo humido inter frutices et ad sepes frequens.

94. H x OSCYAMUs.

Cal. 5-dentat. persistens. Cor. infundibiliformis. Stam. inclinata. Caps. operculata bilocul. polysperma. Spermoph. centrale in medio dissepimento.

1. H. niger: fol. radicalib. pinnatifido-siniatis, caulin. amplexicaulib. sinuato-angulatis, cal. inermib. sessilibus, cor. reticulatis. S. I. 138. H. A. I. 28 .

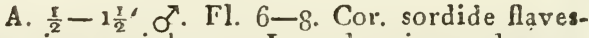
cens intus violacea. In ruderatis et ad pagorum sepes crebro obvius. Off.

95. DATURA.

Cal. angulat. tubulos. deciduus. Cor. infundibuliform. plicata. Caps. 4-valv. inferne 4-locul., superne 2-locul. dissepiment. valvaribus, spermoph. 4, geminis oppositis in mediis dissepiment. minoribus.

1. D. Strammonium: fol. ovat. angulato-dentat. glabris, capsulis ovatis spinosis, spinis aequalibus erectis. S. I. 138. H. A. IV. 7 .

A. $\frac{1}{2}-3^{\prime} \odot$. FI, 6, 7, 8. Cor. alba. In ruderatis et ad sepes pagorum frequens. Off. 
96. $s$ O L A N U M.

Cal. 5-partitus. Cor. rotata interdum plicata. Anth. conniventes subconnatae apice por. geminis dehiscentes. Bacca 2-locul. polysperma.

\section{* Caule fruticoso.}

1. S. Dulcamara: caule fruticoso scandente iner. $\mathrm{mi}$, fol. integris, inferiorib. cordato-ovatis, superioribus plerumque hastutis, flor. corymbosis, co. rymbis dichotomis intrafoliaceis. H. F. 17.

u. vulgaris. 3. palustris. \%.rupestris. H. A.2-15" T. Fl. 5-9. Cor violacea rarius alba. In prais $\alpha$, paludibus $\beta$, muis $\gamma$ frequens. Off.

\section{* Caule herbaceo.}

2. S. tuberosum: caule herbaceo inermi, rad. tuberifera, fol. impari pinnatifidis, pinn. inaequalib. altern. minutis, racem. corymbos. terminalibus, peduncul. articnlatis, cor. 5-angulatis. S. I. 139.

A. I-千'24. Fl. 6-S. Cor, violacea $\nabla$. albs.

In agris undique cultum.

3. S. nigrum: caule herbaceo inermi angulato glabro, fol. ovat. dentato-angulat. integerrimisve glabris, racem. cymos. intrafoliaceis. S. I. 139. H. A. II. 40 .

A. $\frac{T}{4}-I_{\frac{\pi}{2}}{ }^{\prime} \odot$. Fl. $6-9$. Cor. alba. Baccae nigrae. In ruderatis et agris admodum vul. gare. Off.

4. S. villosum (Lamark): caule herbaceo inermi 
tereti hirto, fol. ovat. dentato-angulat. villoso-pubesccntibus, racem. cymos, intrafoliaceis pedunculatis. S. I. $13 \mathrm{~g}$. H. A. II. $4 \mathrm{I}$.

A. $\frac{T}{4}-1^{\prime} \odot$. Fl. 6-9. Cor. alba. Baccae croceae. Praecedente rarins iisdem locis. Stadt. vod. Frankf. u. Coutb. Thor, etc.

\section{I. YCIUM.}

Cal. subcampannlat. 3-5-dencat. persistens. Cor. infundibiliform. fance stam. barbatis clausa. Stigm. subcapitat. 2-fidum. Bacca 2-locul. poIysperma.

3. L. barbarum: ram. deflex. spinosis, fol. lanceolat. basi attenuatis, inferiorib. fasciculatis, sıperiorib. alternis, peduncul. cal. 2-3-fido longioribus, stam, corollae limbum aequantibus. H. F. is.

A. $1 n-15^{\prime}$ T. F1. 5-10. Cor. sordide-lilacina. Baccae favo-rubrae. In solo sabuloso sepes format. Vor d. Hallisch. u. Oranienburger Thor etc.

99. $\mathrm{RHAMN}$ M.

Cal. urceolat. 4-5-fidus. Cor. 4-5-petala calyci inserta stamina includens, interdum o. Bacca 2-3-sperma.

1. $R$. cathartica: spinis terninalibus, fol. subrotund. v. oblongo-ovat. serrat. dentibus incurvat. apice glandulos. 7-nerviis, petiol. glabris, pedun- 
cul, calyce longioribus, flor. 4-fid. plerumque dioi. eis, bacc. globosis. H. F. ' . H. A. V. 43.

A. $6-15^{\prime}$ T - t. Fl. 5,6. Cor viridis. Bacca nigra. In sylvis, pratorum marginibus etc. frequens. Off.

2. R. Frangula: ram. inermibus, fol. ovato-oblong. $r$. subovalib. integr. costato venosis, flor. $\not$ 1-gynis. H. F. 20. II. A. V. 44.

A. $8-12$ ' - t. Fl. $=-0$. Cor albida. Bacca nigra. In sylvis humidis et ad sepes. Thierg. Trepi. etc. frequens.

99. VINCA.

Cal. 5.partitus. Cor. hypocrateriformis. Folliculf 2 erecti. Sem. nuda.

1. minor: canle frutescente repente, ram. erect. 4-angularibus, fol. ovali.oblong. margine glabris, Hor. pedicellatis, cal. glabıis. H. F. 29.

A. $1 \frac{r}{2}-2 \cdot 2$. Fol. senipervirentia. Fl. $4-9$. Cor. violacea. In nemoribus Teg. KLl. Thierg.

100. JASIONE.

Cal. communis 10-sepalus v. fidus, proprius 5 -fidus persistens. Cor. rotata profunde 5-partita. Antherae cohaerentes. Caps. cal. propio coronata 2-locul. apice dehiscens subroundo-5-gona. Spermoph medium basale subglobo3. liberum. 1. F. montana: fol. lineari-lauceulat, basi anguse tiorib. undulato-crisp. Lispidis, L. I. S. V. 373. 
A. $\frac{\pi}{4}-1^{\prime} \odot$. Fl, 6-8. Cor, coerulea rarius alba. In solo arenoso undique frequens.

1C. PHYTEUMA.

Cal. 5-partit. persistens. Cor. rotata 5-partita, laciniis linearib. lax. demum reflexis. Caps. cal. coronata $2-3$ locularis, foramine laterali de. hiscens. Spermoph oblong. carnos. Iamina lon. gitudinaliter medio dissepimento affixa.

1. P. spicatum: fol radicalib. et caulin. inferio. rib. cordat. dupliçato-serratis, caulin. summ. lineari-lanceolat. subintegerrimis, spica oblonga elongata, styl. bi-trifid. pilosiusculis, bracteis cor. brevioribus. S. I. 142.

A. $\frac{x}{2}-1 \frac{x}{2} \cdot 4$. FI. 5. Cor. ochroleuca. In sylvis frondosis rarius. Briselang. S.

102. CAMPANUIA.

Cal. 5-10. (rarius 4-) fid. persistens. Cor. campanulata (rarius rotata) 5-fida. Filam. basi dilatata. Caps. cal. cornnata $2-3$ (et 5-) locularis, foraminib. lateralib. dehiscens. Sper. moph. fungos. medium in axillis dissepimen. tor. longitudinaliter affixum.

1. C. rotundifolia: glabra, fol, radicalib. cordat, reniformibusve ovat. oblongisve serratis, caulin. superiorib. linearib. integerrimis, calycis laciniis subulat. dein, reflexis, pan. subsecunda S. I. 142. 
A. $\frac{1}{4}-1,24$. Fl. $6,7,8$. Cor. cyanea. In agris fertilibus, siccioribus graminosis ubivis obvia.

2. C. patula: lirtella, fol. radicalib. ovat. oralibusque petiolat. crenatis, caulin. superiorib. lanceolat. sessilibus, laciniis cal. lanceolato-subulat. erecto-patentibus, pan. patula. S. I.142.

A. $\frac{\text { r }}{2}-1 \cdot 2$. Fl. 5-9. Cor. cyaneo - violascens saepius alba. In campis, sylvis, pratis frequens.

3. C. Rapunculus: hirtella, fol. undulatis, radicalib. ovato-lanceol. basi attennatis, caulin. lanceolat sessilibus, laciniis cal. lanceol. subulat. utrinque 1-den. tat. erecto.patentibus, racemo subpaniculato S.I.1 43. A. '’ '․ Fl. 8. Cor. cyanea. In solo pinguiori. haud procul ab aquis. Weifss. S.

4. C. persicifolia: glabra, fol. crenato-serratis, radicalib. oblongo-ovat. basi attenuatis, canlin. superiorib. lineari-lanceol. linearibusque sessilibus, laciniis cal. lanceol. patulis, flore solitario aut racemo terminali paucilloro. S. I. 143.

A. $\frac{\mathbf{x}}{2}-1 \frac{\mathrm{r}}{2} \cdot 2$. Fl. 5, 6, 7. Cor. magna cyaneo-violascens. In sylvis frondosis lrequens e. c. Papenb. Jungfli, etc.

5. C. glomerata: hirto-villosa, fol. ferrulatis, radicalib. caulinisque inferiorib. petiolatis oblongo-ovato. lanceolat. subcordatis, caulin. superiorib. lanceolat. sessilibus, laciuiis calycin. Lan. 
ceol. erect. adpressis, for. conglomerat. sessilibus bracteatis. S.1 144 .

A. $\frac{1}{4}-2$ '2!. Fl. 6-s. Cor. cyanea. In col. libus gramiiosis et in sylvis. Teg. W. Temph. Papenb. S.

6. C. urticifolia (Schmidt): hispida, fol. grosse duplicato serratis, radicalib. et canlin. inferiorib. petiolat. cordatis, caulin. superioribus orato-laneeolatis sessilibus, calycis hispidi laciniis ovato-acuminatis, peduncul, axillarib. solitariis, dein cernuis. S. J. 14 .

A. $\frac{x}{2}-2,2$. Fl. 7,8 . Cor. cyanea. In humidis graminosis et sylvis rarins. Papenb. W. Friedrt. S.

7. C. Trachelium: hispida, fol. ovato-lanceol. grosse duplicato-serratis, inferiorib. longius petio. lat. subcordatis, superiorib. subsessilibus, calycis hispidi laciniis ovato-acuminatis, peduncul. axillarib. terminalibusque subuifloris. S. I. 145.

Antecedeni similis isdemque locis obvia. Teg. Schöncb. Reineckend. IV.

8. C. bononiensis: incano-pubescens, fol. crenato. serratis, inferioribus petiolat. cordato-lanceolatis, superiorib, ovat. acuminat, sessilibus, calycis gla. briusculi laciniis brevib. lanceol. ciliat. reflexis, racem. terminalib. subpaniculatis. S. I. 146.

Cunlis teres. 24. FI. 7, S. Pistillum longitudine parrae corollae. Inter frutices et in sylvaticis raxior. Schulzendorf. Walter. S. 1.1. 
9. C. rapunculoides: hirto-scabra laut subglabra, fol. inaequaliter serrat. lanceolato-ovatis, radicalib. et caulin. inferiorib. cordatis, superiorib. ses. silib. basi parum attenuatis, calycis laciniis lanceol. reflexis, racem. terminalib. secundis. S.I. 146.

A. $1-3^{\prime} 24$. Rad. repens. Caulis subaingulosus Fl. 6-8. Cor cyanea. In agris argillaceis r. calvareis et in collibus inter frutices rarius. Rüdersd. S. 103. RIBES.

Cal. sup. 5-fidus. Cor. 5-petala cum staminibus calyci inserta. Sty. 2-fidus. Bacca cal, coronata polysperma spermoph. 2 lateralib. oppositis. * Inermes. (Ribesia.)

1. R. rubrum: trunco erecto, fol. obtuse 5-lobis epunctatis, racem. nutantibus, cal. plan. glabris, bracteis peduncul. brevioribus, bacc. glabris. $H$. F. 24. H. A. JII. 25.

A. 4-5' T. Fl. 5. Cor. Alavo-virens. Bacca rubra. In sylvis et ad margines pratorum. frequens. Thierg. Panck. etc.

2. R. nigrum: trunco erecto, fol. acuminat. 3 v. 5-lob. subtus punctatis, racem. lax. declinatis, cal. campanulato glabro, bracteis peduncul. brevioribus, bacc. glabris. H. F. 26. H. A. IIJ. 26.

A. 4-5' 万. Fl. 5. Cor. Alavo-virens. Bacca nigra. In nemoribus liumidiusculis. Thierg. '́eg. Trept, etc. 


\section{- Aculeatae. (Grossulariae).}

3. $R$. Grossularia: ram, rect. paululum recurvatis, aculeis stipulaeformib. solitariis gemin. $\nabla$. ternis, fol. 3-lobis, petiol. eglanduloso-pilosis, peduncul. sub-1-floris, bracteis 2 discretis, bacc. hirsutis H. F, 27.

A. $2-4^{\prime}$ 7. Fl. 4. Cor. Alavo-virens Ad sepes, in sylvis et nemoribus hinc inde. Thierg. Jungfh, etc.

$$
\text { 104. LONICERA. }
$$

Cal. sup. 5-fidus. Cor. 1-petala, fere 2-labiato. tubulosa, margine irregulari Stigm. capitatum Bacca 2-locul. polysperma.

1. L. Periclymenum: caule volubili, fol. deciduis glabr. subtus pallidiorib. omnibus distinctis, flor. capitat. terminalibus, cor. ringentibus. H. F. I4. H. A. II. 38 .

A. $6-8^{\circ}$ 万. Fl. $6-8$ Cor. pallide-purpurea. Bacc. rubrae. In sylvis hinc inde. Thierg. Briselang.

105. HEDERA.

Cal. 5-dentat. germen cingens ad basin usque deciduus. Cor. 5-petala. Bacca sicca basi ca. lycis 5-gona cincta 5-sperma, sem. 2-3 haud raro abortivis.

1. H. Helix, fol. sempervirentibus, inferiorib. 10. 
batis, floralib. ovat. acuminat. venosis, umbell. erectis convexis. H. F. 27.

Caulis scandens T. Fl. S. 9: Cor. Havo-virens. Baccae nigrae. In sylvis hinc inde. Thierg. Papenb. etc.

\section{ILLECEBRUM.}

Cal. 5-partitus persistens. Cor. o. Squamulae 5 filiformes. Stam. 2. fertilia (basi connata) Stig. ma capitatum. Caps. 1-locul, 5-valv. 1-sperma, semine centrali basali.

1. I. verticillarum: caulib. prostrat. filiformibus, fol. ovat. petiolat. glabıis, flor. verticillatis, cal. 5. gon. aristatis. S. I. 148 .

A. 2-s" $\rightleftharpoons$. Fl. 8. Cill, albus. In solo arenoso humido passim. Reineckend. Charlottb. Grunw. Friedıf. Gesumdbrumnen.

\section{THESIUM.}

Cal. 1-phyllus $1-5$ fidus laciniis basi barbatis. Nux (v. Achaen.) 1-locul. evalv. 1-sperma cal. coronata. Sem. centrale basale.

1. T. Linophyllum: caule erecto subramoso, flor. paniculat, basi 1 - 3 -bracteatis, bracteis inaequa. libus, paniculae ramis linc indebractea foliiforni instructis, muce oval. S. I. 34 \%

a. humile is. vulsare \% ramosum. S. 1. 1. A. I -2 . Rad. liguosa. Fol. lineari-lanceolata. 
F1. 5, 6. In collibus graminosis. Thierg. Spand. Wr. Rnmmelsb. Kih. Papenb. Kalkb.

2. T. ebracteatum (Hayne): caule erecto subsimplici, flor. racemos, basi unibracteatis, bractea fo. liiformi, peduncul. ceterum aphyllis, nuce sub. globoso-conoidea. S. I. $15^{\circ}$.

A. $\frac{r}{2}-1 \cdot 24$. Rad. repens. Fol. lineari-lanceolata FI. 5. In siccis graminosis, Thierg. Jungfh. Kalkb.

\section{ORDO IT. DIG YNI $A$.}

109. ULMUS.

Cal. 4-s-fidus. Cor. o. Samara circumalata 1-sperma.

1. U. campestris: ram. laevibus, annuis et juniorib. glabris, fol. duplicito-serra . basi inaequalibus, flor. subsessilib. conglnmerat. 5-andris fructib. glabris. H. F. 29. II. A. III. 15.

A. 50-100' t. Fl. $3-4$ antequam fol. erumpunt. In plateis, ad vias, prope pagos et in ipsis passim.

2. U. suberosa (Ehrhardt): ramis suberoso-alatis, annuis et juniorib. glabris, fol. duplicato-serrat. basi subaequalibus, flor. subsessilib. conglomerat. 4-andris, samar glabris H. F. 30. H. A. III. 16.! ๙. parpifolia. $\beta$. grandifolia H. I.,. A. 50-200' 
t. Fl. 3 , 4. ante folior cruptionem. In sepibus, ad vias etc. frequens.

3. U. effusa (IVilldinow): ram. laevibus, ramul. annuis et juniorib. pubescentibus, fol. duplicatoserrat. basi inaequalibus, flor. longe pedunculat. 6-S-andris, samar. ciliatis. H. F. 3o. H. A. III. 17. \%. oblongata. $\beta$. rotundata. $\%$ acuminata.

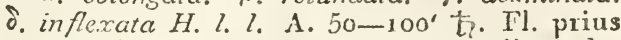
quam fol. erumpunt 3, 4. In nemoribus pla. teis, pagis etc. frequens.

109. SALSOIA.

Cal. 5-partit. v. 5-sepal. mutabilis persistens. Cor. o. Achaen. membranaceum cal, mutato inclusum.

1. S. Kali: herbacea patula hirta, fol. subulat. mucronato-spinosis, cal. solitariis, appendicibus explanat. coloratis. L. T. S. V\}. 225.

A. $\frac{\mathrm{T}}{2}-1$. $\odot$. Fl. 7, 8. In camp. arenosis hinc inde frequens. Wedding. vor d. Hallsch. Th, Weg n. Weifsens. etc.

110. CIIENONODIUII.

Cal. 5-partit. persistens. Cor. ก. Achaen. 1. sup. membranaceum cal. immutato connivente tectum.

* Foliis angulosis.

1. C. bonus Henricus: fol, triangulari-hastat, iz- 
tcgerrimis, 'picis composit. glomerat. aphyll. axillarib. terminalibusque. L. R. S. VI. 252.

A. $\frac{1}{2}-1 \frac{\pi}{2}{ }^{\prime} 2$ \&. Fol. pulverulenta. Fl. 6-8. In pagis, ruderatis, ad sepes, muros etc. frequens.

2. C. urbicum: fol. 3-angularib. sinuato-dentatis, spicis composit. terminalib. axillaribusque confert. strictissim. cauli approximat. longissim. subaphyllis. S. I. 152.

A. $1-1 \frac{T^{\prime}}{2} \odot$. Fol. non pulverulenta. Fl. 7,8 . In ruderatis, ad muros, sepes etc. obvium.

3. C. rubrum: fol, ovato-rhomboidalib. obtusiu. scul. inaequaliter profunde sinuato-dentatis, spicis composit. glomerat, erect. subfolios. axillarib, fol. brevioribus. S. I. 153.

A. $1-2^{\prime} \odot$. Fl. ?, s. Flanta plus minusve rubet. In agris, ruderatis, fimetis et circa pagos vulgaris.

4. C. murale: fol. ovat. inaequaliter sinuatodentat. acut. nitidis, racen, cory mbos, axillarib. terminalibusque aphyllis, caule ramoso patulo. S. I. 153.

A. $\frac{\pi}{2}-1 \frac{\pi}{2} \odot$. Fol. niiida. Fl. 7, 8. In viis, ad muros etc. obvinm.

5. C. album: fol, rhomboideo-ovat. erosis sub. dentatisve, superiorib. oblong. integerrimis, racem. composit. subloliosis S. I. 153.

Chenopodium viride. IV. $p_{0} n_{0}$ 30g. 


\section{A. $\frac{\pi}{4}-3^{\prime} \odot$. Fl. 7, 8. In agris ad vias in} ruderatis copiosissimum.

6. C. hybridum: fol. subcordato-ovat. acuminat. angulato - dentatis, racem. divaricato-paniculat. apbyll. terminalib. axillaribusque. L R.S. V. 259. A. $1-3^{\prime} \odot$. Fol. laete viridia. Fl. 7, 8. In locis praecedentium vulgare.

7. C. glaucum: fol omnib. oblong. repando-dentat. subtus glaucis, spicis glomerat. aphyll, axillarib. terminalibusque. L. R. S. VI. 261,

A. $1-1 \frac{1}{2} \bigodot^{\circ}$ Fl. 7, \&. In campis ruderatis etc. frequens.

* * Foliis integerrimis.

8. C. Vulvaria: fol. rhombeo-ovat. integerrimis, racem. conglomerat, axillaribus terminalibusque aphyllis. S. I. 155.

A. $\frac{1}{2}-1^{\prime} \odot$. Caul. prostrati. Fol. canescedti-pulverulenta. Fl. 7. S. Planta foetidissima in ruderatis fimetis etc. frequens.

9. C. polyspermum: fol. ovat. obtus, integerrimis, caule decumbente, panicul. dichotom. aphyll. axillarib. terminalibusve, cal. fructifer. patulis. L. R. S. VI. 263 .

A. $1-2 \cdot \odot$. Fl. $6-$ S. Fol. laete viridia. In agris cultis et in ruderatis rarius. Schoeneb. Wiv. Teg. Kth. Spand. S. Tafsd. F. B. 
I11. ATRIPLEX.

Polygamum. Cal. ̧. 5-phyllus, ㅇ. 2-phyll. fructiferus persistens demum-increscens. Achaen. membranaceum cal. clauso tectum.

1. A. roseum: caule herbaceo divaricato, fol. ovato-triangularib. inaequaliter dentat. incanis, cal. fructifer. 4-angularib. dentatis. S. I. 157.

A. $I-2^{\prime} \odot$. Fl. 6, 7. Tota planta albido. incana. In solo sicciore et in ruderatis. Hallisch. Th. Spand. etc.

2. A. hortense: caule erecto berbaceo, fol. triangularib. dentat. concoloribus, cal. fructifer. ovat. reticulat. integerrimis. L. R. S. VI. 282 .

A. $1-4^{\circ} \odot$. Fol. subpulverulenta. Tota planta Aavescens. In ruderatis, fimetis et oleraceis passim.

3. A. hastatum: caule erecto herbaceo, fol. triangulari-hastat. profunde.dentat. concoloribus, cal. fructifer. deltoideis sinuato-dentatis. S. I. 156.

A. $\frac{1}{2}-2^{\prime} \odot$. FI. 7,8 . In ruderatis et ad sepes rarius. Strahl. Kih. Schöneb. S.

4. A. patulum: caule herbaceo patentim ramo. - so, fol. delioideo-lanceol. subhastat. subdentatis, cal. fructifer. denticulat. disco muricatis S. I. 156. A. 1-1 $\frac{\pi}{2}{ }^{\prime} \odot$. Fl. 7, 8. In ruderatis, fime. tis, ad vias, et sepes frequens.

3. A. angustifolium: caule herbaceo, ram, diva 
zicatis, fol. lancenl. integerrimis, inferiorib. ha. stat. subdentatis, cal. fructifer. hastato-lanceolat. integerrimis. S. I. 157 .

A. praecedentis $\odot$. Fl, 7, 8. In locis antecedentis copiosissimum.

112. HERIARIA.

Cal. 5-partit. acut. persistens. Cor. 0. Squamulae 5 cum calycis laciniis et staminibus alternantes. Achaen. cal. tectum.

1. H. glabra: caulib. prostrat. adpresso-pilosis, fol. oblique-ovatis, glomerul. axillarib. multifloris. S. I. $15 \%$ H. A. II. 37 .

Planta tota lutescenti-viridis. A. $2-8$ " 2 . Fl. 6-8. Flor. parvi. In agris arenosis frequens.

113. CUSCUTA.

Cal. 4-5 fid. v. partitus. Cor. urceolato-cam. panulata globosave limbo 4-5-fido persistens. Ncct. squamae $4-5$. ad basin staminum v. 0. Caps. 1-2-locul, basi circumscissa, locul. 3-3-spermis.

3. C. europaea: flor. saepius 4-fid. subsessilibus, cor. fauce nuda, stigm, acut. filiformibus. L. R. S. VI. 199.

Caulis scandens aphyllus, glaber. filiformis, albido-rubicundus, plantas alias circumnectis 
et verucis albis in is retinetur. Flor. glo. merati, rubicundi. In fruticetis, ad sepes. Strahl. Th. Spand. etc.

2. C. Epithymum (Murray): Hor. 5-fid. sessilibus, stam. basi squama crenata munitis, stigm, acutis. L. R. S. VI. 201.

Habitus partium minor atque in antecedente. In collibus siccioribus et ericetis rarius. Jungfh. S. I. 158 .

\section{CENTIA NA.}

Cal. 4-5-8. partit. persistens. Cor. 1-petala 4-8 fida inferne tubulosa. Caps. teres I-locul. 2. valv. polysperma, valv. intus undique seminiferis. v. utrinque spermoph. longitudinali instructis.

1. G. Pneumonanthe: caule simplici tereti, fo!. lanceolato-linearib. obtusis, Hor. axillarib. terminalibusque pedunculatis, cor. campanulat. 5-fidis, laciniis acuminatis. S. I. 158.

A. $\frac{r}{2}-1 / 2$. Fl. 6, 7. Cor. cyanea plicata. In pratis humidiusculis. Friedrf. Heiligens. etc.

2. G. uliginosa (Willdenow): caule ramoso 4-gono basi subalato, fol. ovato-lanceolatis, cal. subaequalibus, cor. hypocrateriform. fauce barbat. sub. 5-fidis, laciniis acutis S. I. 158.

A. $1-4$ " $\odot$. Fl. 7, 8. Cor violacea. In prat. siccioribus, ad margines paludum. Grunw. Junfh. Cöpn. Th, etc. 
3. G. campestris: caule ramoso 4-gono, fol. ovato-lanceolatis, cal. inaequalib. laciniis 2 maximis, cor. hypocrateriform. fauce barbat. 4-fidis, laciniis obiusiusculis, S. I. 159.

A. 1-3" $\odot$. Fl. 7, 8. Cor. violacea. In pratis rarius. Zwischen Fasanerie u. Grunw. Chamisso. S. 1. 1.

\section{CYNANCHUM.}

Cal. 5-partit. persistens. Cor subrotata 5.partita. Nect. Corona duplex: exterior 5-20-loba, interior cuculli 5 biloculares. Folliculi 2. 1-lo. cul. longitudinaliter dehiscentes, spermoph. su. turali. Sem. marginata apice desmate instucta. (Massae pollinis ventricosae.)

1. C. Vincetoxicum ( $R$. Brown): caule erecto, cor. imberbibus, pedicell. umbellae simplicis pedunculo communi triplo longioribus, nect. exter. so 5.lobo. S. I. 16o. H. A. VI, 30.

Asclepias Vincetoxicum. $I$.

A. 1-2'2\%. Rad. tuberculosa. Fol. ovata acuminata. Cor. alba. In collibus graminosis et in sylvis acervatim Cöpn. Heide. Papenb. Kalkb. etc.

116. ERY Y G I M.

Flor capitati. Receptac. paleaceum. Involucra sub. spinosa. Cor. inflexae. Fruct. paleaceo-squamati. 
1. E. campestre: fol. radicalib. subternatis, lobis pinnatifidis, laciniis ovatis, caulin. auriculatis, caule paniculato, involucr. integriuscul. lineari-lanceol. pungentib. capitula (rotundata) superantibus, paleis subulatis. I. R. S. VI. 332. H. A. II. 1.

A. $\frac{I}{2}-1 \cdot \frac{I}{2} \cdot 24 . \mathrm{Fl} .6-8$. In agris et pratis rarius. Weg nach Nauen. Meyer.

I17. S:A NICULA.

Umbella capitata. Involucra oligophylla. Flose. polygami. Cal. persistentes. Fruct. oblongi solidi ecostati setis uncinat. v. glochideat. obsessi. 1. S. europaea: fol. inferiorib. palmatis, lobis trifid. inciso-serratis, flosc. omnib. sessilibus. L. R. S. VI. 339.

A. $1-1 \frac{1}{2}^{\prime} 24$. Fl. 5, 6. Cor. rubicunda. In sylvis frondosis humidiusculis passim. Blu. menthal. Kth. Zwischend.Kalkbergen $u$. Woltersd. S.

118. H Y DROCOTYエ

Umbellae imperfectae. Fruct. sutura dorsoque angustis, hoc 3-costato, lateribus compressis subrotundi, cortice plerumque reticulato-ve. noso. (Fol. subrotunda.)

1. $H$. vulgaris : fol. peltat, orbiculat. sublobato. to-crenatis, umbell. capitat. sub-5-floris, L. R.S. VI. 344 .

Caulis repens. Fol. Iucida. 24, Fl 5, Cor. 
alba et incarnata. In pratis humidis et in pa. ludibus frequens. Thierg. Grunw. Jungfh, etc.

\section{IIMPINELA.}

Involucr. nec universale nec partiale. Fruct. ovati solidi glabri, cost. 5 obtusiusculis, vallecul. planinscul, vittatis.

1. P. Saxifraga: glabra v. pubescens, fol pinna. tis, pinn. plerumque sessilibus, radicalium subro. tund. inciso-serratis, superiorum caulin. linearib. integris, raro amborum pinnatifid. v. subpinnatis, IH. A. VII, 2,

a. alba. B. nigra. H. 1. I. A. 1-2' 4. Fl. 7, 8. Cor: alba. In agris, viis, pascuis undique. Off.

2. P. magna: glabra, caule sulcato, fol. pinnatis, pinn. inferiorib. petiolatis, radicalium ovat. gros. se-serratis, superiorum caulinor. lanceolat. pinnatifido-incisis. H, A. VII. 21.

A. 2-3' 4. Fl. 7, 8. Cor. alba. In pratis et humidis sylvarum rarius. Friedrf. Briese. lang S. Papenb.

\section{SESEIT.}

Involucr. universale o, partiale oligophyllum, interdum 1-phyllum. Fruct. ovati solidi, costis 5 acutis, vallecul, sulcatis striatis. (Rami vir. gati. Folia decomposita, foliol, angustis.) 
1. S. dubium (Schkuhr): caule laevi vaginato stricto superne ramoso, vagin. foliorum laxis, infim. pendulis, fol. bipinnatis, foliol. linearib. subtrifid. acutiusculis, involucr. setaceis, universali oligophyllo, partialib. polyphyll. umbellas aequanti. bus. L. R. S. VI. 399.

Selinum Chabraei. Kth. 78.

A. 1-2'. Fl. 6, 7. Cor. alba. In pratis humidis prope fossas sparsim. Jungfh. vor dem Schlesischen Th. Schöneb. Fasanerie.

121. SISON.

Involucr. oligophylla v. o. Fruct. ovatus solidus dorso 3-costatus, vallecul. convexis, latusculis contractis, sutura excavata. (Fol. varia.)

1. S. Podagraria (Sprengel): caule sulcato ramo80 , fol. bi-triternatis, foliol. oblong. serrat. basi inaequalibus, inferiorib. binatis, involucr. nullis, L. R. S. VI. 408 .

Aegopodium Podagraria $L$.

A. $2-3^{\prime} 24$ Rad. repens. Fl. 5, 6. Cor. alba. In hortis, sylvis, dumetis humidiusculis hine inde frequens, Thierg. Scliöneb. Panck. etc.

122. C A R U M.

Involucr. 1-phyllum. Involucella o. Fruct, oblon. go-prismaticus, cost. 3 dorsalib. obtusiusculis, vallecul. sulcatis striatis, sutura lata utrinque costata. 
C. Carvi. H. A. VII. 19. I. W. I. 1470.

A. $\frac{\pi}{2}-3^{\prime} \sigma^{\nearrow}$. Rad. subfusiformis. Caul. fistu. losus, striatus. Fol bipinnata, pinn. oppositis, pinnul. multifidis, laciniis acutis Petioli basi ventricoso-vaginantes. Fl. 5, 6. Cor. alba. In pratis et pascuis frequens. Off.

\section{CNIDIUM.}

Involucr. sub-o. v. 1-phyllum. Fruct. ovati solidi, cost. 5 acut. subulatis, vallecul. sulcatis striatis.

1. C. Silaus (Sprengel): caule angulato, fol. supradecompositis, foliol. pinnatifidis, laciniis remotiuscul. lanceol. nervos. subcanaliculat. cuspidatis, involucro obsoleto. L. R. S. VI. 416.

Peucedanum Silaus. H. A. VII. 5.

A. 1-4. 24. F1.6, 7. Cor. Alavescens. In pratis inter frutices et in sylvis subhumentibus obvium. Thierg. Strahl. Trept. etc.

\section{OENANTHE.}

Involucr. o. Involucella polyphylla. Flosc. saepe radiantes polygami, Fruct. prismatici, cost. 5 acut. $\nabla$. obtusis apice cal, pistillisque coronati. 1. O fistulosa: stolonifera, caule fistuloso-sub. simplici, fol. radicalib. bipinnatis, foliol. plan. cu. neat. lobatis, caulin. pinnat. filiformibus. L. R.S. VI. 418.

A. $2-3^{\prime} 24 . F l, 5,6$, Cor, alba ₹. rubicun. 
da. In paludibus, pratis, fossis undique co. piosa.

2. O: Phellandrium (Lamarch): caule ramoso fistu. loso sulcato, fol. subtripinnatis, foliol. divaricat. orat. inciso-dentatis, peduncul. oppositifoliis, involucro nullo. L. R. S. V1. 425.

Phellandrium aquaticum. H. A. T. 40.

A. $1-5^{\prime} \sigma^{7}$. Fl. 5, 6, 7. Cor alba. Achaen. 2 ovata dorso convexa, costis marginalibus sub-3-gonis, vallecul. 4 1-vittatis. In lacubus, fossis, stagnis undique. Off.

$$
\text { 125. APIUNI. }
$$

Involucra et involucella o. Fruct. ovati solidi, cost. 5 obtusiusculis, vallecul. suturisque planis. 1. A. graveolens: caule sulcato ramoso, fol. pinlnatis, foliol. caulin. cuneiformib. incisis, umbell. subsessilibus. L. R. S. VI. 429. H. A. VII. 24. A. 1-3 $0^{\top}$. Fl. 7, 8. Cor. albida. Cultum in agris his illisve locis.

2. A. Petroselinum: caule ramoso angulato, fol. decompositis lucidis, foliol. inferiorib. ovato-cuneat. trifido-dentatis, superioribus lanceolat. integriusculis, involucell. filiformibus. L. R. S. VI. 429. H. A. VII. 23.

A. $1-3^{\prime} 0^{\top}-24$. Fl. 6, 7, 8. Cor. sublutescens. His illisve locis in agris cultum. Off. 


\section{IIE U M.}

Involucr. o. Involucella oligophylla. Fruct. prise matici, cost. 5 acutis, vallecul. planis, sutura excavata.

1. M. Foeniculum (Sprengel): caule superne ra. moso, fol. biternatis, foliolis lineari-filiformibus glauc. pinnatifido trifidis, involucell. nudis, L. R. S. VI. 433.

Anethum Foeniculum. H. A. VII. 18.

A. 2-f' o', 24. Fl. 6, 7. Cor. Alava In agris cultis et oleraceis rarius. S.I.167. OEE.

127. CICUTA.

Involucr. subnullum. Fruct. ovati solidi, costis 5 , cortice in valleculis prominulo.

1. C. virosa: fol. 3-pinnatis, foliol, subtern. lanceolat. acut. serratis, umbell. oppositifoliis, involucell. lineari-setaceis. L. R. S. VI. 4;2. H. A.I. 37. ๙. latifoita. 3. angustifolia. A. 2-4'24. Rad. loculosa. F1. 6, 7. Cor: alba. In paludibus, fos. sis stagnis et ad fluminum ripas $\mu_{\text {. undique. }}$ ß. Jungfh.

128. AETHUS A.

Involuc. o. Involucella dimidiata. Fruct. ovati so. lidi, cortex in costas 5 acutas prominulas tur. gescens.

2. A. Cynapium: L. R. S. VI. 454. H. A. I. 32. 
A. $\frac{p}{2}-2^{\prime} \sigma^{\top}$. Rad. fusiformis. Fol. inferiora pinnato-supradecomposita, foliol incisis, laciniis lancenlatis, supra npacis subtus nitentibus. F1. 6, 7. Involucella lineari.lanceolata umbel. lis longiora. Cor. alba, In ruderatis ad sepes muros etc. frequens.

\section{TIY Y SEIINUM.}

Involucra et Involucella Fruct. plani, cortice in dorso 3 costas obtusas exhibente, sutura plana. 2. T. palistre (Hoffmann): caule teretiusculo sub. angulato ramoso, fol. ternato-supradecompositis foliol. opposit. pinnatifidis, lacinulis lanceol. albido-mucronatis. L. R. S. VI. 464 .

Selinum palustre $L_{\text {. }}$

A. $2-3^{6}$ 4. Fl. 6-8. Cor albido-lutescens. In pratis paludosis et in sylvis humidis undique frequens. Thierg. Jungfh. etc.

$$
\text { 130. DAUCUS。 }
$$

Involucr. pinnatifid. s. pinnatum. Flor. subradian. tes. Fruct. oblongi, costis 5 aculeatis, angulis interposit. setosis $v$. pariter aculeatis.

1. D. Carota: hispidus, fol. 3-pinnat, opacis, fo. liol. pinnatifid. laciniis lanceol. cuspidatis, involucr. involucellisque pinnatifid, umbellam subaequantibus, aculeis rect. fructuum latitudinem aequantibus. L. R. S. VI. 473. H. A. VII. 2.

$$
\text { A. } \frac{\pi}{2}-3^{\circ} \text { 万. F1. 5, 6, 7. Cor. albida. Rad. }
$$


napiformis. In solo argillaceo aprico, in syl. vis etc. frequens. Off.

$$
\text { 131. TORIII8. }
$$

Involucr. obsoletum. Flor, aequales. Fruct. ovats costati undique setis aculeisve tecti.

1. T. Anthriscus (Gmelin): strigosa, caule erecto ramoso, fol. bipinnatis, foliol. oblong. inciso.ser. ratis, terminali elongato, involucr. minimis. L. R. S. VI. 482 .

Caucalis Anthriscus H. A. I 36.

A. $\frac{1}{2}-4^{\circ} \sigma^{\top}-\odot$. Fl. $6-8$. Cor. rubella v. albida. Ad vias, sepes in sylvis etc. frequens.

2. $T$. helvetica: scabra, caule diffuso ramoso, fol. 2-pinnatis, foliol. ovat. inciso-pinnatifid. serratis, extremo elongato, involucro nullo. L.R.S. VI. 483.

Caucalis arvensis. L.

A. $1 \frac{1}{2}^{\circ} \odot$. Rad. subfusiformis. Fl. 6, 7. Cor. albo-flavescens. In agris argillosis invenit $R$ ebentisch S. I. 171 .

132. SCA NDIX.

Involucr. 0. v. ejus loco folium caulinum. Fruce. pyramidat. rostrat. 5-costatus, nucleo dimidiam fructus longitudinem aequante.

I. S. Pecten: caule aspero, fol. ternato-decompositis, foliol. linearib, abbreviat. multifid. acutis, fructib. scabriusculis. L. R. S, VI. 501. 
A. $1-1 \frac{\mathrm{T}}{2}$. $\odot$. FI. 5. Cor: albida. In agris argillosis inter segetes rarius. Hallsche $\mathrm{Th}$. Weg n. Rüdersd. W. S. I. 171.

133. MYRRHIS.

Involucr. o. Fruct. pyramidat. rostratus, costis acutis; pistillis coronatus.

1. M. Uuibosa (Sprengel): radice tuberosa, caule maculato inferne hirto superne glubro geniculis tumido, fol. supradecomposit. glabriusculis, foliol. lincarib. multifidis, involucell. glabr. cuspi. datis L. R. S. VI. 513 .

Chaerophyllum bulbosum. II. A. T.32,

A. 1-6' б. Rad. napiformis, subramosa. Fl. 6, 7. Cor: alba. Circum pagos et in ipsis in solo argillareo. Kalkb. Wilimersd. Brisel. S. Charlotib. Temph.

I. M. temula (Gacrtner): caule aspero maculato, genicul. tumidis, fol. subbipinnat. utrinque hirtiusculis, foliol. ovat. oblong. incis. denticulatis, involucell. reflex ciliatis, umbell. nubilibus cernuis. L. R S. VI. 514.

Chaerophyllum temulum. H. A. I. 34.

A. 2-4' ${ }^{\prime}$. Rad. subfusiformis Fl. 5. Cor. alba. In ruderatis, ad sepes etc. frequens.

334. GIIAEROPIIIIUM.

Involucr: subnullum. Fruct. conic, ecostat. gla. ber evitlatus, sutura sulcata. 
1. C. sativum (Bauhin): caule glabro, fol. 3 par. tito-decompositis, foliol. ovat. pinnatifidis, laciniis obtusiusculis, peduncul. axillarib. pubescentib. subsenis. L. R. S. VI. 520.

Scandix Cerefolium. H. A. VII. t. 14.

A. $1-2^{\circ} \bigcirc$ Fl. 5. Cor alba. In agris cultis et in ruderatis hinc inde.

2. C. sylvestre: caule sulcato striato glabro, ge. nicul. tumidiusculis, fol. 3-partito-decompositis, foliol. ovat. pinnatifidis, laciuul, oblong. obtusiu. sculis, extremis elongatis, umbell. terminalibus, peduncul. glabris. L. R. S. VI. 521 . H. A. I. 33. A. $2-24^{\prime} 26$. F1. 5, 6. Cor. alba. In sylvis umbrosis humidiusculis frequens.

\section{ANTHRISCUS.}

Involucr. subnullim Fruct. conici muricati pistill. persistentibus, sutura sulcata.

1. A. vulgaris (Persoon): caule laevi, fol. ternato.d acompositis foliol. pinnatifidis, lacinul. obtu. sis, petiol. pilosis, umbell. oppositifol. ovato-conicis. L. R. S. VI. 524.

Scandix Anthriscus Kth. Caucalis scandicina $W$. A. $1-2^{\prime} \odot$. Fl. 5. Cor. alba. In ruderatis ad sepes et pagos frequens. Thierg. Charlottb. etc.

136. sIU $\mathbf{M r}$.

Involucra integra. Fruct. subprismaticus, cost. 5 obtusis, vallecul, subconvexis. 
1. S. latifolium: caule angulato, fol. primariis bipinnatifidis, foliol. infim. decussat. pinnatifid. acuminatis, posteriorib. pinnatis, foliol. lanceol. basi inaequalib. argute serrulatis, umbell. terminalibus. L. R. S. VI. 531. H. A. I. 38 .

A. $2-5^{\prime}$ 24. Rad. repens Fl. 5-9. Cor. alba. In fluminibus, stagnis, fossis, paludibus undiquo frequens.

2. S. angustifolium: caule tereti erecto, fol. pinnatis, foliol. incis. ina€qualiter serratis, umbell. pedunculat. oppositifoliis, involucro foliaceo subpinnatifido. I. R. S. VI. 533. H. A. I. 38 .

A. $\frac{1}{2}-2 \cdot 24$. Rad. rerticillatim fibrosa. Fl. $5,6,7$. Cor. alba. In fossis et paludibus satis frequens.

3. S. repens: caule prostrato radicante, fol. pinnatis, foliol. subrotund. angulato-dentatis, involu. cro oligophyllo lanceolato. L. R. S. VI. 534.

A. 2-r̈" 24. Fl. 6, 7. Cor. alba. In locis humid. graminosis ad margines aquarum ra. rius. Weilsensec.

4. S. Falcaria: caule striato, ram. divergentibus, fol. decursive pinnat. glaucescentibus, foliol. lineari-lanceol. argute cartilagineo-serrulatis, inrolucr. subulatis L R. S. VI. 535 .

A. $1-1 \frac{1}{2}$ \%. F1. 6, 7, 8. Cor. alba. In agris argillaceis his illisve frequens. Templ. Spand. etc. 
5. S. Sisarum: rad. tuberosa fusiformi fascicula. ta, fol. pinnat. ternatisque, foliol. subcordato-ob. long. serratis, umbell. terminalibus. L.R.S. VI. 536. A. $2-3^{\prime}$ F1. 6, 7. Cor. alba. In his illisve locis cultum.

\section{CONIUN.}

Involucra et invollucela. Fruct. ovati solidi, cost. 5 obtusis, immaturis crenulatis, maturis undulatis, vallecul. planis.

1. C. maculatum: caule glaberrimo maculato, fol. 3-pinnatis, foliol. lanceolat. pinnatifidis, lacinul. lanceol. subintegris. L. R. S. VI. 545. H. A. I. 34 .

A. 2-4 o $^{\prime}$. Rad. fusiformis, Caul. striatus, maculis purpureis notatus. Involucella de. flexa. F1. 6, 7. Cor. alba. Ad sepes, muros, in ruderatis et agris passim. Charlottb. Weifss. Stadt. etc. Off.

138. IIGUST IOUM.

Involucr, et involucella varia. Fruct. ovato.oblongus, cost. 5 acutis, vallecul. sulcatis.

1. L. Cervaria (Sprengel): caule simpliciusculo, fol. biternatis rigidiuscul. nitidis, foliol, ovat. subsessilib. mucronato.denticulatis, inferiorib. basi pinnatifidis, laciniis rellexis L. R, S. VI. 550.

Athamantha Cervariz. Kth. 79 .

F. B.

II 
A. $3-4^{\circ} 4$. Rad. in collo comosa. Fl. 6, 7 . Cor. albo-rubella. In sylvis frondosis rarius Teg. Friedrf. W. Papenb.

\section{SEII N $\boldsymbol{\text { Ix. }}$.}

Involucra et involucella. Fruct. planissimi ovales margine alati, cost. dorsalibus 3. subprominulis, vallecul. et sutura vittatis.

1. S. Oreoselinum (Scopoli): caule tereti, fol. 3 -pinnatis, petiol. refractis, foliol. remot. ovat. inciso-pinnatifid. divaricat, nitidis, involucr. reAexis L. R. S. VJ. 557.

Athamanta Oreoselinum. H. A. VII. 3. Kih. 79.

A. 1-2. 24. Canl. striatus, dichotomo-ramosus. Fl. 6-S. Cor albido-lutescens, In agrissiccis. Jungfh. Hasenh. Teg. etc.

\section{HERACLEUIT.}

Involucra o. Flor. subradiantes. Fruct. compressi membranaceo-marginati, cost. 3 dorsalib. ob. tusis, vallecular. commissuraeque vittis clavatis. 1. H. Sphondylium: fol, pinnat. bijug. scabrohirsue. dentatis, foliol. lateralib. sinuatis, extremo 3-lobo palmato acutiusculo, for. albid. subdifformibus, fructib. glabriusculis. L R. S. VI. $57^{2}$ II. A. VII. 10.

A. $1-5^{\prime}$ 24. Caul. sulcatus, hirtus. F1. 6. ? Involncella polypliylla. Cor. alba. In pratis graminosis et sylvis bumidis frequens. 


\section{1. PAST VACA.}

Involucra o. Involucella subunlla. Fruct. ovalis compress. plan. marginatus, cost. dorsalib. obsoletis sire potius siris et vitt. interjectis te. nuissimis, suturae 2 magis conspicuis.

1. $P$. sativa: caule sulcato, fol. pinnat. nitid. subius pubascentibus, foliol. oblong. obtus. crenato. dentatis basi incisis, extremo 3.lobo. L. R. S. VI. 584 . H. A. VII, 16.

A. $1-4^{\prime} \sigma^{\top}$ et 4 . Pad. subfusiformis. $\Gamma 1,6,7^{\circ}$ Cor. lutea Ad vias, in pratis etc. hand rara.

2. P. Anethum (Sprengel): caule simplici tereti, fol. decomposit. glabr. glaucis, foliol. lineari-filiformib. integerrimis. L. R. S. VI. 5S7.

Anethum graveolens. H. A. TII. 17.

A. $2-3^{\prime} \odot$. Fl. 6-S. Cor. lutea. In ruderatis, oleraceis etc. passim.

142. ANGEIICA.

Fruct. subcompressus, cost. 3. acut. alatis, vallecul. sulcatis margine nembranaceo. Involscra. 0.

1. A. sylvestris: caule superne cano pubescente, fol. bipinnatis, foliol. ovat. acut. serrat. basi sub. bilobis, flor. albidis. L. R. S. VI. 600. H. A. VII. g. Caul. $1 \frac{1}{2}-4^{\prime}$ teres striatus, purpurascens. Petioli caulem vaginantes, Cor, 24 , alba. Iu H 2 
pratis et sylvis humidis passim. Rummelst. Talsd. Papenb. etc.

2. A. Carvifolia (Sprengel): caule sulcato acutangulo, fol. 3-nato-decompositis, foliol. 3-fido-pinnatifid. oblongo-lanceolat. albido.mucronatis, pistillis fructus reflexis. L. R. S. VI. 606.

Selinum Carvifolia Kth. 78.

A. 1-3' 4. Fl. 6, 7. albida. In pratis humidis ad sepes etc. frequens. Runimelsb. Thierg. etc.

\section{I. ASERPITIUM.}

Involucra et involucella polyphylla. Fruct. ovalis subcompressus, cost. 3. primariis acutis, secundariis alatis.

I. L. pruthenicum: 'caule simpliciusculo subangulato pilis reflexis, fol. ternato-decomposit. subtus hirsutis, foliol. ovato-lanceolat. pinnatifidis, laciniis lanceol. cuspidat. venosis, involucr. ciliat. reflexis. L. R, S. VI. 623.

A. 1-3. 4. Fl. 6, 7. Cor. alba. In sylvis humidis sparsim. Falkenhagener Heide. S. Pdpenb.

\section{ORDO III. TRIGYNIA.}

144. VIBURNUM.

Cal. (brevis) sup. 5-partitus, Cor. sup. 5-fida. Bacca 1-sperma, 
1. V. Opulus: fol. 3.lobis acuminat, acuminatodentatis, superiorib. saepe indivisis, petiol. glabris glandulosis, glandul. depressis medio concav. sessilibus, cym. radiatis, radii florib. sterilib. $\mathbf{H}$. F. 38 .

A. 10-15' T. Fl. 5. Cor. alba. Bacc. rubrae. In pratis sylvisque humidis Thierg. Teg. Fasanerie etc.

\section{SAMBUCUS.}

Cal. sup. 5-partitus. Cor. sup. 5-fida rotata. Bacca 3-sperma I-locularis.

1. S. Ebulus: caule herbaceo, fol pinnatis, foliol. ovato.lanceolatis acut. basi inaequalibus, cym. 3-partitis. H. F. 38. H. A. IV. 15.

A. $3-4^{\prime}$ T. Fl. 6. Cor. albido-rubella. Bacc. nigrae. In sylvis humidis umbrosis rarius. Thierg. IV.

2. S. nigra: caule arborescente, fol. pinnatis, foliol. ovat. acuminat. basi aequalibus, cym. 5-par• titis. H. A. IV. 26. II F. $3 \mathrm{~S}$.

A. 15-20' h. Fl. f. Cor. alba. Bacc. nigrae. In sylvis humidiusculis et ad sepes undique.

\section{CORRIGIOLA.}

Cal. inf. 5-sepalus, sepal. ovat. concar. membranaceis persistentibus, in fructu conniven. 
tibus. Cor. 5-petala. Achaen. rotundato-trigo. unm calyce cinctum.

1. C. littoralis: flor. pedunculatis, cai. marogine membranaceis. L. R. S. I. 6 s3.

A. $2-S^{\prime} \odot$. Fol. obovato v. lineari-lanceulata. Fl. 6, 7 . Cor. alba. In arenosis huni. diusculis. Hasenh. Fricdrchf. TVeils, etc.

\section{DROSERA.}

Cal. infer. 5-fidus persistens. Cor. inf. 5-petala. Siyl. bifidi. Caps. 1-locul. 3-5 valvis. Scm. plura parva valvis affixa, arillata.

1. D. rotundifolia: scap, erectis, fol. subrotundis, stigmatib. clavatis. H. A. III. $2 \%$.

A. 4-6" 24. Fl. 6, 7. Cor. alba. Fol. glandulosa. In pratis turfosis muscosis et marginibus lacumn frequens. Temph. Grunw. Talsd.. Junghfh. etc.

2. D. longifolia: seap. adscendentibus, fol. obovatis, stigmat, emarginatis. H. A. III. $2 S$.

A. 2" 2f. Fl. 6, 7. Cor. alba. Fol. glandu. losa. Antecedente rarius similibus in locis. Grunw. Talsd.

3. D. anglica (Hudson): scap. erectis, fol. spatluulato.lanceolatis, sigmat, clavatis. II. A. III. $29 \cdot$ A. 3"-6"2. Fl. 6, 7. Cor alba Fol. glan. dulosa. In locis antecedentis his illisve fie. quenter obvia. Grunw. Talsd. 


\section{ORDO IV. TETRAGYNIA.}

1.4S. IPA $\mathbb{N}$ Ass $\mathrm{A}$.

Cal. inf. 5-partitus. Cor. inf. 5-petala. Nect. squa mae 5. ciliatae apice globuliferae. Caps, 1-10. cul. 4-valvis, Sem. spermophoris valvularibus affixa arillata.

1. P. palustris: fol. radicalib. cordat. (acumina. tis), nect. multiparticis. L. R, S. VI. 695. H. A. II. 42 .

A. $\frac{r}{4}=24$. Fl. 6, 7, 8. Cor, alba, In prao tis humidis undique obvia.

\section{ORDO V. PENTAGYNIA.}

149. A RIERA.

Involucr. commune polyplyyllum basi scapum involvens. Calyx monophyllus integer, lim= bo plicato scarioso. Cor. infundibuliform. 5-pe. tala. Filam. basi connata, Achaen. membrana. ceum calyce persistente tectum.

1. A. vulgaris (Willdenow): scapo tereti glabro, involucri foliol. exteriorib. acutis, fol. linearib. plan. obtusis. L. R. S. VI. 77 1.

Statice Armeria.

A. $\frac{5}{2}-2,24$. Fl. $5-9$. Cor rubella rarius alba. In campis et collibus siccis, apricis, gra auinosis undique. 


\section{I I N U M.}

Cal. inf. 4-5-sepalus. Cor. inf 4-5-petala. Filam. basi connata. Caps. 5-10-valv. 10-locul., locul. 1-spermis.

* Foliis alternis.

1. L. usitatissimum: sepal. ovat. trinerv. acutis, petal. crenatis, fol. lanceolatis, caule subsolitario. S. 1. 185. H. A. VIII. 17.

A. $\frac{\pi}{2}-1 \frac{1}{2} \cdot \odot$. Fl. 6, 7. Cor. coerulea. Planta frequenter in agris culta. OfF.

* Foliis oppositis.

2. I. catharticum: fol. obovato.lanceol. integer. rimis, caule superne dichotomo, petal. acutis. L. R. S. VI. 756. H. A. VIII. 18.

A. $3-8 "$ ○. F1. 6, 7. Flos terminalis 1. petalis albis. In pratis et pascuis humidiusculis frequens.

3. L. Radiola: fol. orat. integerrimis, caule di. chotomo, ram. fastigiatis, Hor. 4-andris 4-gynis, petal. mucronatis. S. I. 186,

Radiola linoides. L. R. S. III. 517 .

A. 1-3" $\odot$ : Fl. 6, 7. Cor. alba. In agris arenosis humidiusculis frequens. 


\section{HEX A N R I A. \\ ORDO I. MONOGYNIA. \\ 151. BERBERIS}

Cal. inf. 6.sepalus. Cor inf. 6.petala, petal. ungue biglandulosis, Filam. edentata. Styl. o. Bacca sup. 2-3 sperma.

1. B. vulgaris: fol. obovat. setoso-serratis, racem. pendulis, petal. integerrimis. H. F. 42 . H. A. I. 41. A. 6-r: 万. Fl. 5. Cor. Alava. Baccae ru. brae. In sylvis et ad sepes pleiumque culta. Thierg. etc. Off.

\section{A CORUS.}

Spadix subconicus floriferus. Cal. o. Cor. inf. 6.petala. Styl. o. Bacca sup. 3-locul. polysperma, spermoph. 3 angulo interno loculamento. rum longitudinaliter aftixis.

1. A. Calamus: scapi mucrone foliaceo ensiformi spadicem multototies superante. S. I. 187. H. A. VI. 31 .

A. $2-3^{\prime} 2 \%$. Rad. horizontalis. Fol. ensiformia. Fl. 5. Cor. Havescens. In paludibus fossis et lacubus frequens. Pichelsd. Jungfh. etc. Off. 153. ASPARACUs.

Cal. o. Cor inf. 6-partita, petal. 3 inferiorib. apice reflexis. Stigm. 3. Bacca sup.3-locularis, locul. 2-spernis. (Flor, saepe dioici). 
1. A. officinatis: caule herbaceo erecto tereti, Eol. (sctaceis) plerumque fasciculatis, stipul. solitariis basi mucronatis. H. A. VIII. 29.

A. $2-6.24$. Rad, fasciculata. Fl. 6, 7. Cor. campanulata, pallide olivacea. Baccae coccineae. In agris apricis et ad margines sylvarum. Jungth. Thierg. Hasenh. etc.

\section{CONVAIIARIA.}

Cal. o. Cor. inf. 1-petala 4-6 fida. Bacca sup. inferne 3 locularis, superne I-locularis,

* Corollis campanulatis (Convallaria).

1. C. majalis: scapo subtriquetro, fol. oblongoovat. utrinque attenuatis, flor, racemos. secundis. H. A. IlI. 18 .

A. $\frac{1}{2}-\frac{3}{4}$ ' 24. Rad. sobolifera. Fl. 5. Cor. alba, odora. Bacca coccinea. In sylvis frondosis subliumidis. Papenb. Jangfh, etc. Off.

* $\begin{aligned} & \text { Corollis infundibuliformibus. (Poly- } \\ & \text { gonatum.) }\end{aligned}$

2. C. Polygonatum: caule ancipiti, fol. oblongoorat. alteru. amplexicaulibus, peduncul. axillaribus sub-1-floris, filam. glabris, anther, conniventib. pistillum aequantibus. H. A. III. 19.

A. $1-1 \frac{5}{2} \cdot 24$ Rad. cylindrico-articulata, Fl. 5. Cor. alba apice viridis. Bacca nigra. In sylvis nemoribusque hinc. inde. Thierg. Jungfh. Papenb, etc 
3. C. multiflora: caule tereti, fol ovat. altern, amplexicaulibus, peduncul. axillarib. subbiforis, filam. pilosis, anther. rect. pistillo longioribus. H. A. III, 20.

A. $1-1 \frac{1}{2} \cdot 24$. Rad, conico-articulata. Fl. 5. Cor. alba, apice viridis. Bacca nigra. In sjlvis frondosis. Thierg. Jungfh. Friedrf. etc.

* * Corollis rotatis (Majanthemum)

4. C. bifolia: canle angulato suburiphyllo, fol. petiolat. cordatis, flor. racemos. 4-fidis 4 -andris. S. I. 190.

A, 2-8“24. Tad. repens. Fl. 5. Cor. alba. Bacca rubra. In sylvis frondosis humidis frequens. Thierg. Grunw. etc.

155. ANTHYRICUNI.

Cal. o. Cor. inf. 6. petala patens. Filam. filiformia. Caps. sup. 3-locul. 3.valvis, valv. medio septiferis. Sent. numerosa angulo interno lo. culamentorum affixa.

1. A. ramosum: fol. planis, scapo ramoso, oor. planis, pistillo recto. L. W. II. 138.

A. $2-3^{\prime} 24$. Fol linearia. Fl. 5, 6. Flor. subpaniculati. Cor. alba. In collibus et nemo. ribus apricis. Thierg. Jungfh. Kalkb. etc.

2. A. Liliago: fol. planis, scapo simplicissimo, cor. planis, pistillo declinato. L. W. I. 14 1.

A. I $-2^{\prime}$ 24. Fol. linearia, sublanceolata. Fl 
5, 6. Flor. subracemosi. Cor. alba, major quam in antecedente. In agris, collibus et sylvis arenosis. Thierg. Hasenl. Charlottb. etc.

156. OF NTHOGAIUM.

Cal. o. Cor. inf. E-petala persistens. Filam. basi dilatata. Caps. sup. 3-locul, 3-valvis, valv. medio septiferis. Sem. numerosa angulo inter. no loculamentorum affixa.

* Floribus luteis corymboso-subumbellatis. (Pyrrhochiton).

1, O. luteum: bulbo solitario solido, fol. radicali unico, involucralibus $2-3$, peduncul. teret. glabris, stylo tereti glabro staminib. breviore S.I. 191.

O. luteum $\beta$. L. W. I. II. 114. O. sylvaticum. Persoon.

A. 2-3" 24. Fol, glaucescenti-viridia, lan. ceolato. linearia. Fl. 4, 5. Cor. extus glaucoviridis, intus viridi-lutea, petalis anguste ellipticis obtusis. In solo fertiliori nemorum. Vogelsd. S. Schönhausen.

2. O. praiense (Persoon): bulbo nidulante, fol. radicali unico (ravius $2-3$ ), involucralibus $3-8$, peduncul. 3०gon. giabris, stylo 3-gono glabro sta. mina superamte $S$. I. $29 \%$

o. luteum WV. En. I, 367. Kth. 91.

A. $2-3^{\prime \prime} 4$. Fl. 4, 5. Cor. extus virizescens; intus intense lutea, petal.j lanceolat. obtu. 
siusculis. In agris subarenosis. Charlottenb. Panck. Schönh. etc copiosum.

3. $O$ arvense (Persoon): bulbo nidulante, fol. ra. dicalib. 2. involucralib. 5-12, peduncul. teretib. pubescentibus, stylo 3-gono subpubescente stamina superante. S. I. 193.

C. villosum. IV. En. I. p. 363.

A. $\frac{\pi}{2}$ '4. Fl. 4, 5. Petala lanceolata acuta pubescentia. In apris et sylvis graminosis. Schönh. Charlottb, etc.

* Floribus albis corymboso-racemosis. (Ornithogalum.)

4. O. umbellatum: fol. radical. linearibus, flor. corymbosis, peduncul, bracteatis, bracteis pedunculo longioribus, filament. subulatis. S. 193.

A. $\frac{1}{2}{ }^{\prime}$. Fl. 5. Cor. alba. Inter segetes et in locis graminosis. Kreuzberg. S.

157. IIIIUM.

Cal. o. Cor. 6-petala, fere campanulata. Nect. sulcus longitudinalis versus petalorum basin. Caps. sup. 3-valv. 3-locul, valv. filo reticulatim. conjunctis. Sem. numerosa angulo interno loculamentorum affixa.

1. L. Martagon: fol, verticillat. obovato-lanceolatis, for, reflexis, petalis revolutis. H, A. VIII. 28. S. I. 194. 
A. 2-3.24. Rad. bulbosa. Fl. 6, 7. Cor. brunescenti-purpurascens, maculata. In collibus sylvarum fiondosarum. Papenb.

158. ALIIUM.

Spatha multiflora, arida. Cal. 0, Cor. 6-petala. Caps. sup. 3-locul. 3-valv., valv. medio septiferis. Sem. numerosa angulo interno loculamentorum aftixa.

* Scapo folioso, umbella bulbifera. (Scorodon.)

1. A. carinatum: fol. planiuscul, carinat, margise inferne scabris, spatha 2-phylla longissima, umbella depauporata, stamin. omnib. subulatis. S. I. 194 .

A. 1-1 $\frac{1}{2}{ }^{\prime} 24$. Rad. bulbosa. FI. 6, 7. Cor. rubens. In collibus et agris. Kalkb. S. Spand. W. Jungfl.

3. A. vineale: fol. teretib. fistulos. glabris, spaLha marcescente decidua, umbella multiflora, stamin. tricuspidatis. S. I. 195.

A. $1-1 \frac{1}{2} \cdot 24 \cdot$ Rad. bulbosa. F1. 6, 7. Cor. purpurea. In agris arenosis undiqne.

* Scapo nudo, fol. planis, umbella non bulbifera. (Moly)

3. A. angulosum: scapo ancipiti, fol. linearibus canaliculatis subtus subangulatis, umbella fastigiata, stamin, subulatis. S. I. 195. 
A. $x^{\prime} 2$. Rad. bulbosa. Fl, 6, 7. Cor. purpurascens. In sylvis acerosis passim. Teg. S.

* * Caule nudo, fol. fistulosis, umbella non bulbifera. (Cepa.)

4. A. Cepa: scapo fistuloso basi inflato fol. teretibus longiore, stamin. alterne 3-fidis. S. I. 195.

A. 1-1 $\frac{\pi}{2}{ }^{\prime}$ 4. Rad. bulbosa. Fol. 6, 7. Cor. virescenti-albida. In agris his illisve culta.

\section{JU NCUS.}

Cal. o. Cor.6-petala. Stigm.3. Caps, sup. 3-10cularis 3 valvis, valv. medio septiferis. Sem. numerosa margini centrali dissepimentorum affixa. (Folia plus minusve teretia.)

* Culmo basi vaginato, vaginis subaphyllis.

x. 7. communis (Meyer): aphyllus, culmo laevi viridi rigidiusculo,' corymbo suprafastigiato supradecomposito inflexo, corollae laciniis lanceol. acnminatis caps, obovata retusa sublongioribus, staminib. tribus. Mejer Syn. 12. S. I. 196.

Funcus conglomeratus et $\mathcal{F}$. effusus. Kih. 94.

a. conglomeratus. $\beta$. effusus. $S$. A. $1-2^{\prime} 4 \%$

Rad crassa horizontalis. Vaginae fuscescentipurpurascentes. Fol. fistulosa. Fl. 5, 6. In locis humidis et paludosis undique frequens.

2. F. glaucus (Ehrhardt): aphyllus, culmo stristo glauco tenaci, corymbo suprafastigiato supradecomposito, corollae laciniis lanceolato-acuminatis 
longitudine capsulae ellipticae obtusae mucronatae. Meyer Syn. 13 S. I. 196.

Priori similis 24. Partes omnes glaucescunt. Vaginae inferiores obscuriores nitentes, Fl.5,6. In solo argillaceo udo frequens.

-3. F. filiformis: subaphyllus, culmo debili pal. lido, corymbo suprafastigiato subsimplici, corol. lae laciniis lanceolat. acut. longitudine capsulae subglobosae cbtusae pallidae. Meyer Syn, 17.S. I.197.

Habitus gracilior atque in 7 . communi. 4. Vagina summa saepe colorata. Fl. 6, 7. Cor. magna. In pratis ad fossas exsiccatas rarius. Zw. d. Hallisch, u. Cottbuss Th. Ruthe, S. 1.1. * Culmi vaginis foliosis, foliis teretibus nolulosis.

+ Floribus hexandris.

4. 7. obtusiflorus (Ehrhardt): fol. culmeis dense nodulosis strictis, corymbo suprafastigiato supra. decomposito divaricato, corollae laciniis subaequalib, obtus. capsulam triquetro-ovatam obtusam sub. aequantibus. Meyer Syn. 20 S. I. 197.

A. $\frac{1}{2}-3: 4$. Color partium pallidus. Fl. 6, 7. Cor. laciniae apice membranaceae. In uliginosis turfosis rarius. Talsd. S.

5. F. fusco-ater (Schreber): erectus, fol. culmeis tereti-compress, nodulosis, corymbo suprafastigiato erecto, corolla laciniis subaequalib, obtusis cap- 
sula elliptica mucronulata brevioribus, Meyer Syn. 22. S. I. 199.

Funcus articulatus $\alpha$. W.p.n.391. et f. sylvaticus Kth. 96. (ex parte.)

A. $\frac{x}{2}-1 \frac{x}{2}, 24 . F l, 6,7$. In locis graminosis humidis undique.

6. F. lampocarpus (Ehrhardt): adscendens, fol. culmeis nodulos, tereti-compressis, corymbo suprafastigiato subpatente, corollae laciniis subaequalib. lanceolatis, exteriorib. acutis, interiorib. obtusis capsula triquetro-ovata acuta mucronata brevio. ribus, Meyer Syn, 23. S. I, 198.

funcus articulatus. Kth. 96 .

A. $\frac{r}{2}-1 \cdot 2$. Fl, 6, 7. Cor. major quam in praecedente. In locis humidis, ad fossas, in pratis etc. frequens.

7. F. acutiflorus (Ehrhardt): fol, caulin, remot. nodulos. tereti-compress. strictis, corymbo supra. fastigiato supradecomposito, corollae laciniis acuminatis, interiorib, sublongioribus capsula trique. tra acuminato-rostrata brevioribus, Meyer Syn, 24. S. I. 199 .

funcus sylvaticus Kth. 96. et $\mathcal{F}$. articulatus $\gamma$. p. n. 391. (ex parte.)

A. $3 \frac{\pi}{2}-2^{\prime} 4$. Fl, 6, 7. In locis antecedentis haud rarus.

$+\dagger$ Floribus triandris.

F. $B$. 
8. F. uliginosus (Roth): culmo filiformi, fol, subnodulosis strictis, for. in corymbi suprafastigiati irregularis ramis subcapitatis, corollae laciniis subaequalibus acutis capsula oblonga obtusa subuniloculari brevioribus, stam, tribus dimidiae corol. lae longitudine. Meyer Syn. 29. S. I, 199.

Funcus subverticillatus. Kth. 96 .

A. $\frac{1}{2}-1^{\prime} 24$. Fl. 6, 7. Planta variabilis in locis humidis ad fossas etc. frequens.

*** Culmi vaginis foliosis', foliis canaliculatis plunisve.

+ Floribus triandris.

9. F. capitatus (IVeigel): annuus, culmo foliisque subsetaceis strictis, corymbo suprafastigiato capitulis subcomposito, corollae laciniis cxteriorib. acuminatis, interiorib. breviorib. acutis capsulam ovatam acutam trilocularen superantibus. Meyer Syn. 36, S. I. 200.

A. $3^{\prime \prime}-\frac{r_{2}^{\prime}}{}{ }^{\prime} \odot$. Fl. 6, 7. Cor. laciniae albidae $\nabla$. purpureo-fuscae. In solo humido, ad margines lacuum etc. frequens, Grunw. Temph. TVeifss. etc.

t + Floribus hexandris.

10. F. bufonius: culmo subramoso aut simplici filiformi, fol. planiuscul. angustissimis, corymbo suprafastigiato elongato, nor. distantibus composito, corollae laciniis Janceol, acuminatis, exterio- 
rib. longioribus capsulam oblongam obtusan su. perantibus, staninib. plerumque scx. Meyer Sym. 39. S. I. 201.

A. $\frac{r}{4}-\frac{r}{2}$ - - Fl. 6, 7. Planta variabilis is solo liumido fregtrentissima.

11. J. Tenageia (Ehrhardf): annuins, culmo subramoso ant simplici, fol. plan. angustissimis, co. rymbo suprafastigiato elongato, florib. distantib. composito, corollae laciniis ovat. acut. mucronatis capsulam subglobosam parum superantibus. Nieyer Sjn. 41. S. I. 201.

A. $2-12 "$ ○. Fl. 6, 7. Corollae laciniae in margine membranaceo et nerro nedio albidae. In soló limoso ad margines lacumm. Scliöneb. IV. Vieilis. Templ!

12. F. Bulbosus: culmo subsimplici compresso, fol. cauliculatis, corymbo suprafastigiato suberecto, corollac laciniis obtusis, incrioribus subbrevioribus dimidiam capsulam subglobosam vix superanibus, stylo brevi. Mcyer Sym. ffo. S.I 202.

A. "-12" 2h. Taginae loxae margine albido membranaceo. Fl. 6, 7. In locis humidis ex subhumidis frequens.

13. F. :quarrosus: culno simplici anguloso stricto, fol. duris canaliculatis, corymbo suprafastigiato composito, corollae laciniis ovalo-lanceolat. 
acutis capsulam obovatam obtusam aequantibus. Meyer Syn. 48. S. I. 202.

funcus Sprengelii. W. p. n. 39;. t. 4. $f$. $S$.

A. $\frac{\mathrm{r}}{2}-1 \cdot 24$. Fl. 6, 7. Rad. caespitosa. Cor. margine albida, in medio fusca, nitens, In prat. et loc. turfosis humidis et siccioribus fre. quens. Grunw. Jungfh. Charlontb. etc.

1 6o LUZULA.

Cal. o. Cor. 6-petala. Stigm. 3. Caps. sup. 1-lo. cularis 3-valv. Sem. 3 fundo mediae capsulae affixa. (Fol. plana.)

* Corymbi rumis unifloris.

1. L. pilosa (Wilıdenow): corymbo suprafastigiato composito denum divaricato, flor. solitariis, caps. obtusis. S. J. 203.

funcus pilosus. L. Luzula vernalis D. C. Fl. fr. III. 160 .

A. $\frac{r}{4}-1 \cdot 24$. Rad. caespitosa stolonifera. Fl. 4, 5. Cor. castaneo-nigrescens, nitida. In sylvis nemoribusque siccis graminosis frequens. * * Corymbi ramis spicigeris.

2. L. campestris (De Candolle): corymbo suprafastigiato simplici, ram. spiciger. dein patentibus, corollae laciniis ovato-lanceol. acuminatis, interiorib. longioribus, rad. stolonifera repente, S.I. 203. Kth. 98. (ex parte.)

Funcus campestris, W.p. n.398. (ex parte.) 
๙. pumila, $\beta$. elata. A. 2-12" 24. Fl, 4,5. Corymbus pauciflorus (spicis 5-10.). Cor. castanea, nitens. Undique frequens in siccis graminosis et sylvis.

3. L. erecta (Desvaux): corymbo suprafastigiato subcomposito, ram. spiciger. erectis, corollae laciniis ovato-lanceolatis, exteriorib. acuminat. longioribus, interiorib. obtusiuscul. mucronat. brevioribus, rad. fibrosa. S. I. 104.

Funcus campestris W.p.n.398. L. campestris $K t h$. 9S. (ex parte.)

๙. pallescens. 6. conferta. \%. micrantha. A. 2-12" 24. Fl. 4-5. Corymbus multiflorus (spicis 5-20). Cor. pallide-castanea.

161. PEIIIS.

Cal. inf. 12-dentatus, dentibus altern. minoribus. Cor. inf. 6-petala v. o. Caps. sup. 2-locul. evalvis polysperma, spermoph. carnoso in medio dissepimento.

1. P. Portula: fol. petiolatis obovato-cuneatis, flor. axillaribus solitariis. S. I. 205.

Caules prostrati. (- Fl. 7,8 . Cor. rubicunda v. o. Planta tota plus minusve rubet. In solo humido ad fossas et lacuum margines hinc inde. Temph. Weilss. 'Teg. etc. 


\section{ORDO III. TRIG Y IA.}

162. SCHEUCIIZERTA.

Cal. inf. G-partitus, Cor. o. Caps. 3-6 unilocular. subdisperm. 2-valves. Sem. fundo capsulae affixa.

1. S. palustris. L. W. II, 263.

A. $\frac{r}{2}-1^{\prime} 2$. Rad. geniculata, repens. Fl. 4,5 . Flor. subracemosi. Fol. canaliculata inferne vaginantia, apice compressa. In prat. turfosis Grunw. Jungfli. Tafsd.

$$
\text { 163. TतICLOCIIIN. }
$$

Cat. inf. 3-sepalus. Cor. inf, 3-petala (calycifor- mis). Caps. basi dehiscens 3-6-cocca.

1. $T$. palustre: caps, elongato-clavatis trigonis.

A. $1-1 \frac{r}{2} \cdot 24$. Rad. fibrosa. Fol. linearia, canaliculata. Flores racemosi. Caps. 3-locularis. In pratis humidis frequens. Charlottb. Teg. Friedrf. etc.

2. $T$. maritimum: capsulis oblongo-subrotundis hexagonis.

Differt praeterea $a b$ antecedente habitu partium majore et capsul. 6-locularibus. In sinilibus locis rarius provenit. Talsd. Teg.etc.

$$
\text { 164. COICHICUI. }
$$

Cal. o. Cor. inf. infundibiliform. radicalis, tubo longissino, limbo G-partito. Caps, sup. 3-10. 
clil, inflata 3 -ralyis, suturis introrsum versis dehiscens. Sem. numerosa angulo interno loculamentorum affixa.

1. C. autumnale: fol. erectis lineari-lanceolatis margine planis, styl. corolla brevioribus H. A: V. 45. S. I. 206.

A. $\frac{1}{2} \cdot 24$. Rad. bulbosa. Fol. lineari-lanceolata. Fl. 9. sine foliis. Cor. lilacino-violascens. In pratis. Falkenhagen. Mundt. S.1.1. Off.

$$
\text { 165. TOIIEIDIA. }
$$

Cal. inf. 3.fidus. Cor. inf. 6.petala, Caps. superae 3 basi junctae 1-locular. 2-valv. polyspermae. 3. T. palustris (Hudson): flor. bracteat, racemo. sis, involucro pedicello adhaerente. S. I. 207.

Anthericum calyculatum. L, Helonias borealis. L. IV. II. 247.

A. $\frac{1}{3}-1^{\prime}$ 24. Rad. caespitosa. Fol. ensifor. mia. Fl. 6, 7. Racemi terminales, Flor. Havescentes. In locis uliginosis et udis sylvarum passim. Charlottb. Chamiss.o. Jungfh. S.

\section{RUMEX.}

Cal. 3-phyllus, Cor. 3-petala, petal. conniventibus et achaen. triquetrum tegentibus. - Floribus hermaphroditis (Lapathum).

1. $R$. crispus: fol. lanceol. crenulat. margine undulato.crispis, verticillis approximatis, summis 
aphyllis, valvul. ovat. integris unica plerumque granifera. S. I. 207.

A. 1-3' 4. Fl. 7, 8. In pratis, locis nemorosis, ad fossas etc. frequens.

2. R. Hydrolapathum (Hudson): fol. oblongo-lanceol. utrinque acuminatis, margine crenulato pli. no, verticill. approximatis, summis aphyllis, valv. subrhombeis oblongo-ovat. integris graniferis. $\mathrm{S}$. I. $2 n 8$.

Rumex Britannica W. p. n. 402.

A. 3-5' 2. Fl. 7,8. In fossis et paludibus ad lacuum et fluminum ripas undique.

3. R. Nemolapathum: fol. inferiorib. cordato-lanceolatis, superiorib. lanceol. margine crenulat. planis, verticill. remotis fere omnibus folinsis, valv. lanceolat. obtus. integris graniferis. S. I. 208.

A. $1-3$, Fl. $6,7.4$. In sylvis subhumidis ad sepes etc. fiequens.

4. R. maritimus: fol. lanceolato-linearib. acutis integerrimis planis, verticill. approximat. foliosis, valv. ovat. acuminat. setaceo-dentatis graniferis. S. I. 208.

A. $\frac{T}{2}-1^{\prime} \odot$. Fl. 7.8. Planta tota lutescens. Ad lacuum et fossarum margines hinc inde frequens. Weifss, Pulvermühlen, Temph. etc. 5. R. obtusifolius: fol. ovato-oblong. subcrenulat, subcrispis, inferiorib. cordat. obtusis, superio. 
rib. utrinque attenuatís, summ. lanceolatis, verticillis approximatis, summis aphyllis, valv. ovat. acuminatis dentatis unica granifera. S. I. 209.

Rumex acutus. W. p. n. $40 \%$ \%

A. 2-4' 4. Fl, 6, 7. In locis siccis et hiumidis, in sylvis, ruderatis etc. frequens.

* Floribus dioicis (Acetosa).

6. R. Acetosa: fol. oblong. sagittatis, valv. graniferis. S. I. 209.

A. 1-3'24. Fl. 5. In pratis humidis et siccis in sylvis etc. copiosus. Off.

7. R. Acetosella: fol. lanceol. hastatis, valv. grano destitutis. S. I. 210 .

A. $\frac{x}{2}-1,2$. Fl. 6, 7. Planta tota rubet. In agris et sylvis sterilioribus frequentissimus.

\section{ORDOIV. POLYGYNIA. 167. A II SIIA.}

Cal. infer. 3 fidus persistens. Cor. inf, 3-petala. Achaen. plura receptaculo convexiusculo im. posita.

1. A Plantago: Hor. verticillat. composito-paniculatis, achaen. obtuse-trigonis, fol. acuminatis basi non cordatis. S. I. 210.


A. $1-3^{\prime} 4$. Fl. 6, 7. Cor. albido.coerulescens. In paludibus et aquis frequens. Off. 
2. A parnassifolium : flor, verticillat. compositepaniculatis, achaen. uncinat. dein aristatis, fol. acutiuscul. cordatis. S. I. 2 II.

Iabitus antecedentis, sed minor. 24. Fol. minora saepe natantia. Fl. 6, 7. Cor. major albida. Ad margines aquarum et in ipsa aqua rarius. Gıunw.

3. A. natans: flor: axillaribus et oppositifoliis, achaen. cylindraceis mucronat striatis, fol. basi mon cordatis. S. I. 212.

A raria. 24 . Fl. 5, 6. Cor. albida. In aquis et paludibus frequens. Grunw. Jungfh, etc.

\section{VII. HEPTANDRIA。 \\ ORDO I. MONOGYNIA.}

168. AESCULUS.

Cal, 1-sepal, ventricos. 4-5-dentatus. Cor. $\{-5$. petala irregularis, petal. unguiculat. calyci insertis. Caps, sup. 3-locularis 3-valvis.

1. A. Hippocastanum: fol. 5-7: nis, foliol obovat. acuminat. duplicato-serratis, cor. 5-petalis, unguibus calyce brevioribus, filament. corollae longitudine, anther. pilosis, fructib. aculeatis. II. F. 43. H. A. I. 42 .

A. 40-60 五. Fl. 5. Cor. alba punctis ru. bris. In viis et remoribus frequentissima. Off. 
169. TחIENTALIS.

Cal. 7-sejal. inferus. Cor. 7-petala infera. Caps. sup. 1-locul. eralr. polysperma, spermoph. centrali globoso.

1. T. europaea: fol. lanceolat, integerrimis. L. IV. II. 282.

A. 3-12" 24. Fl. 5, 6. Cor. alba. Staminum numerus variabilis. In sylvis umbrosis rarius. 'regel. Heide. S.

$$
\text { 17O. C.A.LA. }
$$

Spatha I-phylla. Spadix foribus dense tectus. Cal. o. Cor. o. Bacca polysperma, seminib. fundo baccae affixis.

1. C. palustris: fol. cordatis, spatha plana, spadice undique hermaplirodito. L. IV. II. 2go. H. A. IV. 5 .

A. $\frac{r}{2}-1 \cdot 24$. Fl. 6, 7. Spatha alb.x. Baccae rubrae. In paludibus et fossis hinc inde. Teg. Talsd. Grunw.

\section{Cl. VIII. OCTANDRIA. \\ ORDO I. MONOGYNIA.}

171. OENOTHERA.

Cal. 4-fid. deciduus. Cor. 4-petala, Caps, inf. cy. lindrica 4-locul. 4-valvis, valv. medio septi. feris, spermophoro centuali columnari. 
1. O. biennis: fol. ovato-lanceolatis planis, caule muricato-villoso, stam, coulla brevioribus. L. W. II. 306 .

Oragra biemis Gaermer. I. I5g.

A. $2-4^{\circ} \sigma^{7}$. Fl. 6, 7, S. Cor. sulphurea suaveolens. In agris arenosis ad sepes et in versuris frequens.

172. EPIIOBYUM.

Cal. 4-sepal. decidıus. Cor. 4-petala. Caps. inf. cylindrica 4-locul. 4-valvis, valv. medio sep. tiferis, spermophoro centrali columnari libero profunde 4-sulcato. Sem. apice desmate in. structa.

* Staminibus declinatis (Chamaenerion).

1. E. angustifolium: fol. sparsis lineari-lanceolat. integerrint. venos, glabris, flor. in aequalibus $L$. VV. II. 313. S. I. 214 .

A. $2-5^{\prime}$ 24. Fl. 6, 7. Cor, violaceo-lilacina. In sylvis et nemoribus et inter frutices. spar. sim. Papenb. Teg. Jungfh.

* Staminibus erectis (Epilobium).

2. E. hirsutum: caule ramosissimo villoso tereti, fol. opposit, alternisque subamplexicaulibus ova. to.lanceul. argute dentatis ad venas villos. gla. briusculis. S. I. 215.

A. 3-6, 24. Fl. 6, 7, 8. Cor. purpurascenti- 
lilacina. In paludibus et fossis sparsim frequens. Strahl, Treptow. Spandau etc.

3. E. pubescens (Roth): caule tereti subsimplici villoso, fol. altern, oppositisque subsessilib. lanceol. denticulatis utrinque pubescentíbus. S. I. 215.

A. $1-2^{\prime} 4$. Fl. 6, 7, 8. Cor violaceo-rosea. In pratis humidis ad fossas etc. hand in fre. quens. Weifss. Teg. etc.

4. E. montanum: caule teretiusculo subsimplici pubescente, fol. opposit. alternisve petiolat. ovat. acuminat. inaequaliter argute dentatis glabris. S. I. 216 .

Epilobium roseum. W. p. n. 42.. Kth. 105.

A. $1-2^{\prime} 4$. Fl. 6, 7, 8. Cor. lilacina. In sylvis frondosis et inter frutices. Thierg. Jungfh. Papenb. etc.

5. E. palustre: caule tereti ramoso subsimplici glabriusculo, fol. oppotitis alternisve sessilib. oblongo-lanceolat. integerrim. glabriusculis. S. J. 216. A. $\frac{x}{2}-1^{\prime} 4$. Fl. 6, 7. Cor. violaceo-lilacina. Ad margines paludum et lacuum etc. fiequens.

6. E. tetragonum: caule tetragono ramoso glabro, fol. opposit. alternisque sessilib. oblongo. lanceolat. argute denticulat. glabris. S. I. 217.

A. $1 \frac{1}{2}-2,2$. F1. 6, 7. Cor. rosaceo-lilacina. In pratis et paludibus rarius. Ieg. Talsdorf etc. 


\section{VAC CINIU M.}

Cal. 4-5-fid. v. 4-5-dentatus. Cor. urceolata v. campanulata I-petala ore 4-5-fido. Stam. calycis fundo affixa. Bacca inf. 4-5-locul. calyce coronata, locul. 2 ซ. polyspernis.

* Fol. annotinis s. deciduis (Myrtillus).

1. F. Myrtillus: caule angulato, fol. ovatis acut. serrais, peduncul. 1-floris. H. F. 45 .

A. $1-1 \frac{1}{2}$, h. Fl. 5, 6. Cor. rubicundo-alba. Bacca nigrescenti-coerulea. In syivis et nemoribus undique frequens.

2. $Y$. uliginosum : caule tereti, fol. obovat. rotun dat. integcrimis, peduncul. 1-floris. II. F. 46.

A. $\frac{\pi}{2}-1$ h. Fl. 5. Cor. rubicunda Bacca coerulea carne atba. In paludibus turfosis rarissime. Teg. Cliamisso. S. I. 218.

* Foliis sempervirentibus.

3. V. Vitis idaea: caule erecto ramoso, ram. alternis, fol. obovat. integr. revolut. subtus punctatis, racem. terminalib. nutantibus. H. F. 48. H. A. IY. IO.

A. $\frac{1}{2}$, 7. Fl. 5, 6, 7. Cor. alba. Baccae coccineae. In sylvis et nemoribus frequentissima.

174. Oxx coccos.

Cal. sup. 4-fidas. Cor. 4-petala, petal. reflexis. Stam. calycis fundo inserta. Bacca cal. coronata f-locul. polysperma. 
O. palustris: caule filiformi nudo repente, fol. subcordato-orat. margine revolutis. H. F. $4 \mathrm{~S}$.

Schollera Oxy'coccus H.A.IV.18. Vaccinium Oxycoccus. S. $I .219$.

Fl. 5, 6. h. Cor. pallide purpurea. Baccae enccineae. In paludibus turfusis frequens. Grunw. Talsd. etc.

$$
\text { 175. CALLUNA. }
$$

Cal, k-sepalus. Cor duplex, exterior 4-petala, interior 1 -petala campanulata 4-partita. Stam. receptaculo inserta. Antherae 2 -fidae, ante dehiscentiam in conum coalitae. Caps. 4.locu. laris, dissepimentis columnae centrali insertis. C. vulgaris, Salisbury. H. A. IV. 17. S, I. 219. Erica vulgaris. L. IV. II. 373.

A. $\frac{1}{2}-2 \cdot \pi$. Fol. sempervirentia Fl. 7,8 . Cor. lilacino-purpurea. In arenosis campis tt sylvis undique frequentissima.

$$
\text { 176. EnICA. }
$$

Cal. 4-sepalus. Cor. 4-fida. Stam. receptaculo inserta. Antherae bifidae. Caps. 4-locul. 4-valvis, marginibus valvarum intus $\mathrm{flexis}$ dissepimen. ta formantibus.

1. E. Tetralix: fol, quatern. oblong. ciliat. sub. tus tomentosis, flor. nutantibus, umbell. capitat. terminalibus, cal. ciliat. tomentosis, cor. ovatis, anther. aristatis. H. F. 49 . 
A. 1-4' T. Fl. 6-9: Cor. brunneo v. albolilacina. In paludosis turfosis. Schoneb. Spandow TV.

\section{A C E R.}

Flor. polygami. Cal. 5-fidus. Cor. 5-petala v. o. Samarae 2 sup. 1-alatae 1-spermae connatae. * Foliis simplicibus integris.

1. A. tataricum: fol, ovato-cordatis integr. v. et exciso-angulatis, fere duplicato-serratis, racem. erect. corymbosis. H. F. 209.

A. 15-20' 万. (apud nos) Fl. 5. Flor. albi, post folioum evolutionem erumpentes. Rarius. Thierg

$$
\text { * Folïs simplicibus 5-lobis. }
$$

2. A. campestre: fol. 5-lobis, lob. obtus. integerrimis, tribus majorib. cuneiformibus, plus minusve sinuato-excisis, petiol. glabris v. subpubescenribus, racem. erectis. H. F. 211.

A. $20-40^{\prime}$ 而 et $\hbar$. Fl. 5. Flor ex viridi. flavescentes cum folits erumpunt. Hinc inde in sylvis frondosis. Thierg. Teg. etc.

3. A. Pseudo-Platanus: fol. 5-lobis, lobis inaequaliter serrat. subtus glauc. plerumque glabris, juniorib. villoso-pubescentibus, racemis pendulis H. F. 212.

A. $60-80^{\prime}$ T. F1. 5. post foliorum eruptionem. Cor. Alavo-virescens. Ad vias et in sylvis. Thierg. Teg. Stadt. 
4. A. montanum: fol. plerumque 5-lobis, lob. acuminat. inciso-serrat. subtus pubescentibus, racenı. composit. erectis. H. F. 212.

A. pensylvanicum. Du Roi. A. spicatum. Lamarck. A. 14-2n . . Fl. 5. post foliorum evolutionem. Cor. Havo-virens. In nemoribus ra. rius. Thierg.

5. A. rubrum: fol. 5-lobis, excisuris incisis, lob. excis. serrat. basi latioribus, subtus glancescentibus, umbell, paucifloris, germinib. glabris. H. F. 213. A. $40-50$ ' 7 . Fl. $4-5$ ante fol eruptionem. Cal. et Cur. purpur. ascentes. In nemoribus raxius. Thierg.

6. A. dasycarpum: fol. 5-lobis, excisur. sinuatis, lobis subsinuato-excisis, basi attenuatis, subtus glaucis, umbell. paucifloris, germinib. villosis. II. F. 213 .

A. $20-40^{\prime}$ 方. Fl. $4-5$. ante fol. eruptionem. Cor, rubicundo-flavescens, In nemoribus et ad vias hinc inde. Thierg. Linden.

7. A. saccharinum: fol. finuato-5-lobis, lob. grosse dentat. obtusiusculis longe acuminatis subtus pubescentibus, corymb. subpendulis, peduncul. laxis pilosis. H. F. 214.

A. 40-60 万o Fl. 4. Flor. navo-virescentes simul cum foliis apparent. Ad vias et in ne. moribus linc inde obvium. Thierg.

F. $B$.

K 
8. A. platanoides: fol. sinuato-5-lobis, lob. grosse dentat. cuspidato-acuminat. utrinque glabris, co. rymbis erectis, peduncul. strict.glabris. H. F. 215 . S. I. 222 .

A. $60-80^{\circ}$ t. Fl. 4-5. Flor. Alavo-virescentes et folia eodem tempore proveniunt. Ad vias et in nemoribus frequens. Stadt. Thierg. Cliarlottb. etc.

* * Folïs compositis.

9. A. Negundo: fol. ternat. pinnatisque, pinn. ovat. lubato-excis. grosse serratis, flor. dioicis, $\vec{d}$ 4-5-andris. H. F. 2 I 6.

A. $70-80^{\prime}$ 7. Fl. 5. Flor apetali cum foliis proveniunt. Ad vias rarius. Thierg.

\section{ORDO III. TRIGYNIA.}

178. POIYGONUI.

Cal. o. Cor. 5-partita inf. calycina persistens. Achaen. simplex. angulosum 3-quetrum . compressum.

* Caule non volubili.

+ Floribus pentandris $\bullet$. hexandris (Pir. sicaria).

1. P. Hydropiper: caule erecto, fol. lanceolat. nndatis, ochreis ciliatis, racem. spicat. cernuis, flor. 6-andris semidigymis, achaen. 3-quetris, H, A. Y. 20. 
A. 1-2, С. Fl. 7, 8, 9. Cor. alba v. rosea. In locis humidis, ad fossis etc. frequens.

2. P. minus: caule basi repente, fol. lineari-lanceolatis planis, ochrcis ciliatis, racem. spicat. ere. ctis, flor. 5-antiris submonogynis, achaen. obsolete-z-quetris. H. A. V. 21.

A. $\frac{r}{2}-1^{\prime} \odot$. Fl. 7 , 8, 9. Cor. alba v. roses. In pratis humidis ad fossas etc. frequens.

3. P. Persicaria: canle erecio, fol. lanceolato pla. nis, ochreis ciliatis nudicve, racem. spicat. orato. oblong. erectis, pedunculis laevib. aut setoso.scabris, Horib. hexandris semidigynis, achaeniis sab. rotund. compressis mucronatis. S. I. 223. H. A: V. 22 .

Pulyg incanum. L. iN. II. 446. P. lapathifolium var. Auct.

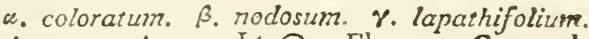

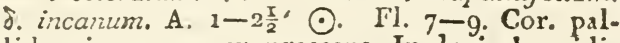
lide virens $\nabla$. purpurascens. In locis humidis agrortum, sylvarum etc. frequens.

4. P. amphibium: caule nutante aut erecto, fol; oblong. lanceolatisve planis, racem. spicat. oratis, flor. 5-andris senidigynis, achaen. triquetris.S. I. 225 .

a. aquaticum, $\beta$. terrestre. Rad. repens 4 Fl. 6, 7. Cor. purpurea, In aquis es paludebus frequens. 
+ Floribus 8-andris, racemo spicato terminali (Bistorta).

5. P. Bistorta: caule simplici erecto, fol. radicalibusin petiolum decurrentibus, racemo subspicato terminali, ochreis oblique truncatis, for. 3-gynis, achaen. 3 quetris. S. I. 225. H. A. V. 19.

A. 1-3' Rad. crassa, fusca. Fol. ovata v. ovato-lanceolata. Fl. 6, - Cor. purpurea. In pratis. Talsd. Friedrf. etc. passim frequens. Off. + + + Floribus 8-andris axillaribus ( $C \mathrm{en}$. tumnodium).

6. $P$. aviculare: caule procumbente $\nabla$. erecto, fol. lanceolat. v. linerri-lanceolatis, ochreis laceris, Hor. axillaribus 5-aut octandris trigynis, achaen. triquetris H. A. V. 23. S. I. 226.

$\boldsymbol{\alpha}$ procumbens, $\beta$. erectum, $\boldsymbol{\gamma}$. ap.hyllum. 4. $\frac{1}{2}-1^{\prime} \odot$. Fl. 6-9. Cor. pallide-purpurea v. alba. In plateis, agris etc. fiequentissimum.

$$
\text { * Caule volubili (Hel.xine). }
$$

7. P. Convolvulus: caule volubili angulato-striato, fol. ovatis cordat, acuminat. glabris. echreis Iruncatis, racem. axillaribus, laciniis calycin. extern، carinat. scabris, carina acuta. S. I. 226.

A. $3^{\prime \prime}-2^{\prime}$ ○. Fl. 6, 7, 8. Cor. rubella. In agris et sylvis frequens.

8. $\boldsymbol{P}$. dumetorum: caule volubili tereti striato, fol. ovat. cordat, acuminat, glabris, ochreis trun. 
catis, racemis axillaribus, laciniis calyc. externis laevibus, carina membranacea. S. J. 227.

Priori simillimum iisdemque locis conspicuum.

* Caule erecto herbaceo, foliis basi incisis (Fagopyrum).

9. P. Fagopyrum: caule erecto subflexuoso inermi, fol. spiculatis, ochreis obliquis, corymbis axillaribus terminalibusque, achaen. integerrimis acutiusculis. H. A. V. 24.

Fagopyrum esculentum (Moench). S. I. 227.

A. $1-3^{\prime} \odot$. Fl. $6-8$. Cor. purpurascens. v. rosea, glandulifera. Cultum satis frequenter.

10. P. tataricum: caule erecto inermi, fol. sagittato-cordat. acuminatis, flor. egland nliferis, achaen. 3-quetr. acutis, angul. repandis. Gaert. II. 1\$2. S. I. 228 .

Fagopyrum tataricum Gaertn. l. lo S. l. l.

Antecedenti simile $\odot$. Cor. non glanduli. fera. Inter segetes prioris rarior.

\section{ORDO IV. TETRAGYNIA。}

$$
\text { 179. PARIs. }
$$

Cal. 4-sepalus. Cor. o. v. 4-petaĺa infera, petal. calycis laciniis angustioribus, Bacca 4-locul. polysperma. 
1. P. quadrifolia: fol. quaternis, flore completo. II. A. III. 7. S. I. 228 .

A. $\frac{1}{2}-I^{\prime}$ Rad subrepens. Fol. ovato-acuminata. 24. Fl. 5, 6. Petala rellexa Havo-viridia. Bacca atro-coerulea. In umbrosis sublumidis. Papenb. Friedrf. Teg. etc.

\section{1हo. ADOXA.}

Cal. inf, 2-4-dentatus basi cum germine con. natus. Cor. calyci imposita inf. 4-5-fida. Bacca calyce semivestita 1.locul. 4-sperma. 3. A. Moschatellina. S. I. 229. L. W. II. 472. A. $2-6 " 2$. Rad. repens, geniculata. Fol. 3-partita et pinnata. Fl. 4, 5. Flor. capitati. Cor. luteo-virescens. Stam. $8-12$. In umbro. is sublıumidis nemorum. Schönh. Fapenb.

\section{EIATIN.}

Cal. infer. 4-sepalus. Cor. inf. 4-petala. Caps. 4-locul. q-valvis, dissepimentis valvis parallelis spermophoro centrali adnatis.

1. E. Alsinastrum: fol, verticillatis. L. W. $\mathbf{U}$. 473. S. I. 229 .

A. 2"-1' $\odot$. Rad. fibrosa. Fol. linearia et ovata, acuta. Fl. $6-8$. Flor. axillares. Cor. alba. Ad margines lacuum et in ipsa aqua. Weils. Temph.

2. E. Hydropiper: fol. oppositis. L. W. II. 472. S. I. 230 . 
A. $2-S " \odot$. Fol. spathulata v. obovata. Fl. 6-9. Flor axillares. Cor. alba v. rosea. Caespites format ad lacuum margines. Wei. fsens. Temph.

C 1. IX. EN N EA N D IA,

ORDO III. HEXAGYNIA.

1S2. BUTOMUS.

Cal. o. Cor. inf. 6-petala. Caps. 6. 1-loculares introrsum dehiscentes. Sem, undique parieti. bus affixa.

1. B. umbellatus. L. WV. II. 491. S. I. 230.

A. $2-5^{\prime} 24$. Rad. subrepens. Fol. linearia inferne 3-quetra. Fl. 6, 7. Flor. umbellati. Cor. ex albo purpurascens striis purpureis. In solo limoso fossarum et lacuum. Weifss. Templ. etc. fiequens.

\section{1. X. D E C A N D R I A. ORDO I, MONOGYNIA.} 183. MONOTROPA. Cal. inf. 4-5-sepalus. Cor. inf. 4-5-petala, pee tal. basi concav. melliferis. Caps. sup. 4-5-lo. 
cul. 4-5-valvis, spermoph. centrali cylindrico. Sem. numerosa arillata.

1. $M$. Hypopitys: racemo multifloro conferto, forifero nutante, fructifero erecto, squamis floribusque extus glabris, flor. lateralib. plerumque 8 andris. A. $\frac{1}{4}-1^{\prime} 24$. Rad. fibrosa, fibrillis soboliferis, scapi ratione habita, debilis, quam ob rem etiam, quantum percipere nobis contigit, arborum radicibus insidere easque fibrillis cir. cumnectere videtur. Scaporum squamatorum basis attenuata, a nonnullis pro ipsa radice habita, obconica est conique apice fibrillis insi. det et lateribus prolem gerit. Fl. 6, 7 . Cor. Havo-fusca, intus ciliata. Staminum aeque ac valvarum numerus variabilis. In umbraculis sylvarum. Thierg. Grunw. Papenb. Cöpuick. Heide.

\section{S4. IEDUM。}

Cal. inf. 5.partititus. Cor. inf. 5-petala expansa. Caps. 5-valv. 5-locul. basi dehiscens. Spermoph. 5 apice adnata. Sem numerosa arillata. х. L. palustre: fol. lanceolato.linearibus margine revolutis, subtus ferrugineo-tomentosis, for. 10-an. dris, racem. terminalibus. H. F. 54.

A. 1-4 7. Fl. 5, 6 Cor. alba. In turfosis humidis. Grunw. Off.

$$
\text { 185. PYROIA. }
$$

Cal. inf. 5-pattius. Cor. inf. 5-petala. Caps. 
sup 5-locul. angulis dehiscens 5-valvis. Spermoph. 5-centralia. Sem. plura arillata.

* Flore solitario terminali.

3. P. uniflora: fol. ovato-subrolund. venosis, pedunculo terminali. unifloro. H. F. 6 ;

A. $\frac{1}{8}-\frac{1}{4}$ h. Rad. repens. Fl. 6, 7, Cor. alba. In sylvis subliumidis. Jungfh. Grunw. $\mathrm{Pa}$. penb. Teg.

* Floribus corymbosis.

2. P. umbellata: fol, verticillat. Ianceolato.obovat. grosse serratis, basi cuneiformibus integris, flor. corymbos, longe petiolat. terminalibus, stam. conniventibus, stylo germini inserto H. F. 63.

Chimophila corymbosa Pursh. I. 300 .

A. $\frac{2}{2}-\frac{3}{4}$ ' h. Fl. 6, 7. Cor. ex albo-purpu= rascens. In sylvis acerosis. Jungfh. Grunw. Teg. etc.

$$
\text { * * Floribus racemosis. }
$$

3. P. secunda: fol. ovatis vo ovali-ellipticis $\nabla$. elliptico-subrotundis breviter mucronatis venosis, racemo secundo, staminib. styloque exserto rectis. H. F. 63 .

A. $\frac{r}{4}-\frac{1}{2}$ h. Fl. 6, 7. Cor. alba. In sylvis frequens. Thierg. etc.

4. P. minor: fol. subrotund. elliptic. ovatisque venosis, racem, vagis, stam. conniventibus, stylo recto incluso. H, F, 63, H, A. IV, 23. 
A. 6-9" h. Fl 6. Cor. rubicunda. In sylvis acerosis et frondosis frequens. Teg. Grunw. Papenb. Köpn. Heide etc.

5. $P$. chlorantha (Scwartz): fol. subrotundis quintuplinerviis, racemis vagis, stam. conniventibus, stylo declinato. H. F. 63. H. A. IV. 22.

P. rotundifolia $W$. p. n. 457. P. media Kth. 115. $P$. asarifolia $S$. I. 235 .

A. $6-9 "$ h. Fol. forma variabilis. Fl. 6. Cor. flavo-virens. In sylvis acerosis et frondosis passim. Jungfh. S. Teg. Grunw.

6. $P$. rotundifolia: fol. subrotund, venosis, ra. cem. vagis, stam. adscendentibus, stylo declinato. H. F. 63. II. A. IV. 28 .

A. $\frac{3}{4}-1 \frac{1}{2}$ h. Fl. 6, 7. Cor. alba. In sylvis subhumidis rarius. Tafsd. S. Grunw.

\section{ANDROMEDA.}

Cal. inf. 5-partitus. Cor. ovata 1-petala ore 5.fido, Caps. 5-locul. 5-valvis, valv. medio septiferis. Sem. numerosa.

1. A. polifolia: fol, coriaceis persistentib. ovalilanceol. v. lineari-lanceol. v. lanceolato-linearib, brevissime mucronat. margine revolut. integris glabris, subtus albo-glancescentibus, peduncul. ter. minalib. aggregatis 1.floris, cor. globosis, stam, apice aristatis. H. F. 61. H. A. III, 22, 
A. $\frac{\mathbf{r}}{2}-\mathbf{x}, \mathbf{T} . \mathrm{Fl} 5,6$. Cor. ex albo purpura. scens. In locis turfosis. Tafsd. Temph. Spaud, Grunw.

187. ARIUTUS.

Cal. inf.5-partitus. Cor. 1-petala ovata, ore 5-fido basi diapliana. Stam. corollae inserta. Bacca 5-locul. 5-sperma.

1. A. Uva ursi: caule procumbente, fol. coriaceis sempervirentib. obovat. rotundat. integris subtus reticulato-venosis. II. F. 6z. II. A. IV. 20.

A. 1-2 $\frac{1}{2}$ T. Fl. 4, 5. Cor. rubicunda. Bacca scarlatina. In sylvis acerosis rarius. Jungfh. Laud procul a nemore Tegeliensi unico loco. Ktli. Rahnsdorfer Heide Chamisรо. Off.

\section{ORDO II. DIGYNIA.}

188. CHRYSOPIENIUI.

Cal. inf. $4-5$. partit. basi germen includens. Cor. o. Caps. sup. 1-locul. semibivalv. polysperma, seminib. basi insidentibus.

C. alternifolium: fol. alternis, rad. repente. S. I. 237 .

A. $3-4^{\prime \prime} 2$. Fl. 4. Fol. radicalia cordzto. reniformia grosse crenata Flor. corymbosi. Cal. Havus. In locis lumidis, ad fossas etc. Thierg. W. Teg. Kalkb. S. Panck. 
189. SAXIFRAG.

Cal. inf. 5-partitus. Cor 5-petala. Caps, sup. 2-ro. stris 2-locul. inter rostra dehiscens polysper. ma, spermophoro centrali crasso.

\section{* Foliis indivisis.}

3. S. Hirculus: fol. caulin. lanceolat. alternis nud. inermibus, caule erecto L. IV. II.649. S. I. 237. A. 6-12"24. F1. 7, 8. Cor. flava. Calyx reflexus. In pratis humidis. Hinter Oranienburg. * Folies lobatis.

2. S. granulata: fol. lobatis, radicalibus renifor. mibus, canliuis cuneiformibus, caule (erecto) ramoso, rad. granulata. H. A. III. 23. S. I. 238. A. $\frac{I}{I}-1$. 4 Fl. 5. 6. Cor. alba In locis graminosis siccioribus nemorum copiose. Ha. senh. Grunv. Thierg Temph. etc.

3. S. Tridactylites: fol radicalib. oblongo-spa. thulat. subintegıis, caulin, ovato-cuneiformib. subintegris aut apice trifidis, caule erecto ramoso, rad. fibrosa. S. I. 238.

A. 2-3" $\odot$. Fl. 4, 5, Cor. alba. In agris argillaceis fiequens. Panck. Schönh. Friedrf. etc.

190. SCIERANTHUS.

Cal. inf. 1-sepal. 5-dentatus persistens. Cor. 0. Achaen. calyce indurato apjce pervio inclusum. 1. S. annuus: calycis fructiferi laciniis acut, mu. 
cronat. margine laterali anguste membranaceis patuks. S. I. 239.

A. 2-8" $\odot$. Fol, linearia, Fl. 6, 7. Planta tota viridescens. In agris frequens. Teg. Panck. IVeifss. Temph. etc.

2. S. perennis: calycis fructiferi laciniis obtusis margine toto late membranaceis conniventibus. S. I. 239 .

Caulis plerumque rubens 4 . Fol. linearia. FI. 5-10. In agris undique.

$$
\text { 191. GYPSOPHILA. }
$$

Cal. 1-sepal. 5-gon. 5-dentatus. Cor. 5-petala, petalis exunguiculatis. Caps. sup. globosa 1-locul. 4-5-valvis. Spermophor, centrale crassum.

1. S. muralis: fol. linearib. planis, cal. aphyllis, caule dichotomo, petal. crenatis, L. W. II. 666. S. I. 240 .

a. matura, E. serotina (G. serotina Kth. 118) A. 2-6" ९. Fl. 7-10. Cor. ex albo purpura-

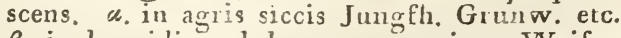

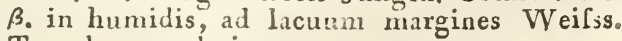
Temph. etc. obvia.

$$
\text { 192. SAIONAFIA. }
$$

Cal. i-sepal. tubulos. 5-dentatus. Cor. 5-petala, petalis unguiculatis ad basin laminae 2-dentatis. Caps, oblonga sup, 1-locul, apice $4 \mathrm{den}$ - 
tibus dein recurvatis dehiscens, Spermoph. centrale erassum.

1. S. officinalis: cal, cylindricis, fol, ovato-lanceolatis. L. IV. II. 667. H. A. II. 2.

A. $1 \frac{I}{2}-2^{\prime} 24$. Rad. repens. Fl. 6, 7, 8. Cor. rubicunda Hortorum plerumque aufuga in coemeteriis, ad sepes etc. passin obvia. Gesundbr, Panck. Weils. Off.

193. DIANTHS.

Cal. 1-sepalus tubulosus 5-dentatus, calyculatas. Cor. 5-petala, petal. unguiculatis. Caps. cylindrica sup. 1-locul apice. 4-5-valvis polysperma. Spermoph. centrale crassum.

- Floribus fasciculutis.

1. D. carthusianorum: hor. fasciculatis, squam. calycin. otat. aristat. glabris tubo brerioribus, involucro oblongo aristato fasciculo breviore, fol. linearib. nervos. glabris. L. W. II. 671. S. I. 241. A. $\frac{r}{2}-2^{\prime}$ 4. Fl. 6-9. Cor, sanguineo-purpurea. In agris grominosis siccis undique irequens.

2. D. prolifer: flor. fasciculatis, squam. calycin. membranaceis ovatis obtusis tubo longioribus, inFolucro flores subaequante, squamis membrana. ceis ovatis, fol. linearib. uninerviis glabris.

A. $\frac{x}{4}-1^{\prime} \odot$. Tl. 6, 7. Cor. parva lilacinopurpurea. In collibus apricis Grunw, $Z$ w. 
Hallsch, u. Leipz. Th, S. Tafsd, etc, hinc inde frequens.

\section{* Floribus solitariis.}

3. D. deltoides: flor. solitariis, squam. calycinis ovato-lanceolat. acut. subbinis subpubescentib. tubo breviorib, fol. linearib. obtusiuscul. subpubescentibus, petal. argute dentatis. S. I. 2 q2.

A. $\frac{I}{2}-1 \frac{1}{9} \cdot 24$. Fl. 6-9. Cor. sanguineo-pur. purea. In locis siccis graminosis undique fre. quens.

4. D. superbus: flor. solitariis subpaniculatis, squam. calycin. orat. acuminat. glabris tubo brevioribus, fol, lanceolat. acut. 3-nervib. scabriusculis, petal. capillaceo-multifidis. S. I. 243.

A. 1-2, 24. Fl. 6, 7. Cor. pallide lilacina. In pratis subhumidis his illisve frequens. Friedrf. Spand, ctc.

\section{ORDO III. TRIGINIA.}

13j. cUCUBAIUS.

Cal. inf. 1-sepal. 5-dentatus. Cor. inf. 5-petala, pctalis unguiculatis. Nect. o. Caps. 3-locul. apice 3-valvis, valvis medio septiferis. Spermoph. centrale crassum.

ג. C. Behen: cal. subglobos, glabr. reticulato renosis, caps, 3.locularibus, cor, subuudis L, W. II. 68 4. 
A. $1-2 / 24$. Fol. oblonga scabriuscula. Fl. 6, 7. Cor. alba. Inter frutices; ad margines nemorum etc. sparsim. Trept. Kalkb. Papenb. Spand. etc.

2. C. Otites: petal. linearib. indivis. undulatis glabris, flor. dioicis, peduncul. glabris, caule paniculato. L. WV. II. 688.

A. $\frac{\pi}{2}-2^{\prime} 24$ Fol radicalia subspathulata. Fl. $5,6,7$. Cor. flavescens. In locis arenosis, apricis collibus, sylvis. Jungfh. Teg. Grunw. etc.

\section{SILENE.}

Cal. inf. 1-eеpal. 5-dentatus. Cor. inf. 5-petala, petalis unguiculatis. Nect. corona ad faucen. Caps. subtrilocul. apice 5-6.valvis. Spermoph. centrale.

1. S, chlorantha: fol. oblong. acut, glabris margine scabris, pan. secunda, Hlor. cernuis, cal. gla. bris, petalis 2-fidis, laciniis lineafibus. S.I. 245 .

A. $1-1 \frac{1}{2} / 2 \%$ Fl. 6, 7. Cor. ex lutescentiviridescens. In collibus sterilibus hinc inde. Spand. Bamberge.

2. S. nutaris: fol. oblongis acuis (radicalibus subspathulatis) pubescentibus, pan. secunda, Hor. nutantibus, cal. viscido-pubescentibus, petal. 2-partitis, laciniis linearibus. S. 1. 2.,5.

A. $\frac{r}{2}-1 \frac{1}{2} \cdot 24$. Rad. subrepens. Fl. 5, 6, 7. Cor. alba. In sylvis frondosis. Hasenh. Jungfh. Papenb. etc, satis frequenter obvia. 


\section{S TEIIA R I A,}

Cal. inf. 5-sepalus, Cor. inf. 5-petala, petal. bipartitis. Caps. 1-locul. 6-valv. polysparma. Spermoph, centrale brevissimum.

1. S. Holostea: caule adscendente 4-angrulo, fol. sessilibus lanceolatis acuminatis margine ciliato. scabris, pan. dichotoma, sepal. enerviis petal plus duplo brevioribus. S. I. 246 .

A. $\frac{1}{4}-\frac{3}{4}, 24$. Caulis subrepens. Fl. 5, 6, 7 . Cor. magna alba. In nemoribus frondosis. $\mathrm{Pa}-$ penb. Thierg. etc. frequens.

2. S. graminea: caule diffuso v. erecto 4 -angulo, fol. lineari-lanceolat, acutiuscul. laevibus, pan. diclotoma pauci v. multiflora, sep. 3-nerviis nunc cor. aequantibus v. superantibus, nunc dimidia cor. brevioribus,

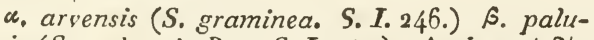
stris (S. palustris Retz S.I. 247.) A. $\frac{1}{4}-1,24$. Fl. 5-7. Cor. alba. In locis humidis et palu. dosis undique frequens. Planta admodum variabilis in florum partibus et foliorum colore ita, ut $S$. gramineam et $S$. palustrem, magna speciminum copia comparata, discernere non possis.

3. S. crassifolia (Ehrhardt): caule erecto 4-angulo, fol. oblongo-lanceolat. obtusiuscul. laevibus, pan. pauciflora 2-toma, sep. enerviis petal. brevioribus. S. I. 247 .

F. $B$. 
A. 2-4" 4. Rad: snbrepens. Fl. 6, 7. Cor. alba parva. In paludibus et locis turfosis. Jungfh. Tafsd.S. Grunw.

4. S. Alsine (Hoffmann): caule procumbente 4 -angulo, fol. sessilib. oblongo-lanceolat. basi ciliatis, pan. 2-3-toma, sep. 3-nerviis petala superantibus. S. I. 2 亿 $\delta$.

A. $\frac{r}{1}-\frac{r}{2}$ (?. Fl. 6-s. Cor. parva alba. in locis humidis frequentissima.

5. S. ntedia (Smith): caule tereti procnmbente 1-fariam piloso, fol. ovat. glabris, inferiorib. petiolatis, flor. alarib. terminalibusque, sepal. subuninerviis petala aequantibus. S. I. 248 .

Alsine media. L. H. A. II. 47.

A. $\frac{1}{4}-2 \cdot \odot$ v. 24. Fl. 4-10. Cor. alba. Planta variabilis undique conspicua.

$$
\text { 197. AREN } \triangle \text { RIA. }
$$

Cal. inf. 5-sepalus. Cor. inf. 5-petala, petalis integris. Caps. 1-locul, apice 3-6-valvis polysperma Spermoph. centrale brevissimum. * Exstipulatae.

1. A. trinervia: fol. petiolat. ovat, acut, $3-5$ nerviis, sepal. obsolete 3-nerv. glabr. carina scabris corollam superantibus, caps. cernuis, sem. laevibus nitidis. S. I. 249.

A. $2-6$ "24. F1. 5-8. Cor. albida. In syl- 
$\nabla$ is frondosis humidis elc. frequens. Thierg. Papenb. Teg. etc.

2. A. serpyllifolia: fol. sessilib: ovat. acut. uninerviis, sepal. sub-5-nerviis pubescentib. cor. superantibus, caps. erectis, scm. tuberculato-scabris. S. I. 249 .

A. 2-6" ○. Fl. 6-8. Cor, alba. In agris copiosissima.

3. A. viscidula (Thuillier): fol, sessilib. Ianceolat. apice subulat. 3-nerr., sepal. 3-nerv. pubescentib. corollam superantibus, caps. erect. trivalvibus, scm. tuberculato-scabris. S. I. 249.

Arenaria tenuifolia. Kth. 123.

a. glabrata. 6. viscosa. A. 1-6" C. F1.5, 6. Cor. alba. In agris siccioribus t collibus frequens.

$$
\text { * * Stipulatae. }
$$

4. A. rubra: fol. sessilib linearib mucronat carnosiuscul. enervibus, stipul. ovat. acuminat. mem. branaceis, sepal. enerr. pubescentib. corollam superantib., caps. subnutantibus, sem. subtubercula. to-scabriusculis. S. I. 250.

A. 1-6" ○. Fl. 5-7. Cor. rubra. In agris arenosis frequens. Temph. Grunw. Jungfh. Teg. etc. 


\section{ORDO IV. PENTAGYNIA.}

199. 8 P ERGUIA.

Cal. inf. 5-sepalus. Cor. inf. 5-petala, petalis in. tegris. Caps. 1.locularis 5-valv. polysperma Spermoph. centrale globosum.

1. S. arvensis: caulib. pubescentibus, fol. opposit. subfasciculat. pubescentibus, sem. subglobos, com. press. scabris. S. I. 251.

A. I-6" Р. Fl. 5-8. Cor. alba. In agris undique obvia.

2. S. pelitandra: caulib. subglabris, fol. opposit. subfasciculat. pubescentibus, sem, subglobos. compressis laevibus, margine albidis nembranaceis. S. I. 252 .

A. I-6" $\odot$. Fl. 4, 5. Cor. alba. In agris et sylvis arenosis. Hasenh. Grunw. Jungfh. etc. frequens.

3. S. nodosa: caulib. subglabris, fol. opposit. aut subfasciculatis laevibus, superioribus abbreviatis, sem. ovoideis subtuberculato-scabriusculis. S.I 252.

A. 4-6" ○. Fl. 5, 6, 7. Cor. alba. In Iocis humidis agrorum sylvarumque frequentissima.

199. CERASTIUM.

Cas. inf. 5-sepalus. Cor. inf. 5-petala, petal. 2-fi. dis. Caps. 1-Locular, polysperma apice dentib. 
5-10 dehiscens. Spermoph. centrale colum. nare.

* Capsulis subglobosis.

1. C. aquaticum: pubescens subviscidum, fol. sessilib. subcordato-ovat. acutis, peduncul. alarib. et terminalib. solitariis, petal. calycem superantibus, caps. subglobosis apice 5 -dentatis, dentib. subfissis. S. I. 253.

A. 1-2, 24. Cor, alba. In locis humidis sylvarum, ad fossas et paludes. Thierg. Jungfh. Friedrf. etc.

* Capsulis oblongis.

2. C. arvense: pubescenti-villosum, fol. lanceolat. obtusiusculis, sepal. 1 -nerviis, nervo medio excurrente, petal. calyce duplo longioribus, rad. repente. S. I. 253 .

A. 3-6" 2\%. Fl. 5, 6. Cor. alba. In agris frequentissimum.

3. C. viscosum: hirsutum subviscid. diffusum, fol. lanceolato-oblongis, cal. oblongis, sepalorum nervo medio fere usque ad apicem procurrente. S.I.254.

Cerastium vulgatum. Kth. 127.

A. 3-8" 24. Fl. 5-8. Cor. alba parva. In agris et sylvis frequentissimum.

4. C. semidecandrum: hirsutum viscidum, caulib. erect, aut basi decumbentibus, fol. ovatis, calycis' 
globoso-ovatis, sepalor. nervo medio ipsis tertia parte breviore. S. I. 254 .

Cerastium viscosum $K_{t h} .127$.

«. cespitosum. 6. erectum. A. 2-4" $\odot$. F1. 5,6 . Cor: alba, parva. In agris, sylvis etc. frequentissimum.

$$
\text { 200. I YCINIS. }
$$

Cal. inf. 1-sepal. 5-dentatus. Cor. 5-petala, petal. unguiculat. 2 -fidis. Nect. corona ad faucem. Caps. 1-5-locular. apice 5-valv, polysperma. Spermoph. columnare.

* Capsulis 1-locularibus.

1. L. fios cuculi: caule pubescente, fol. lanceolat. glabris, petal. 4-fidis, caps. subglobosa. S. I. 255.

A. $\frac{x}{2}-3^{\prime}$ 4. Fl. 5, 6. Cor. purpurea, interdum alba. In pratis et hunidis graminosis undique.

2. L. dioica: caule villoso, fol. lanceolat. villosis, flor. dioicis, petal. 2-fidis, caps, ovoidea basi ventricosa. S.I. 256. H. A. II. 3.

A. 2-3' 24. Fl. 6-9. Cal. $0^{\top}$ cylindricus, 오. inflatus. Cor: alba. In agris ad vias etc. frequentissima.

* Capsulis 5-locularibus.

3. L Viscaria: caule glabro, internodiis superne viscosis, fol. spathulato-lanceolat. glabris, petal. integris, caps. subglobosa. S. T. 256. 
A. 1-3, 24. Fl. 5, 6. Cor rubra. In collibus graminosis sylvarum hinc inde. Teg. Pi. clielsb. Papenb. etc.

\section{A G ROSTEMIIA.}

Cal. inf. 1-sepal. pentagonus 5-dentatus. Cor. inf. 5-petala, petal. unguiculat. integris. Nect. o. Caps. 1-locular. apice 5-valv. polysperma. Spermoph. columnare.

1. A. Githago: sericeo-hirsuta, petal. emarginat, calycem subaequantibus. S. I. 257 .

A. $\frac{1}{2}-3^{\prime} \odot$. Fol. linearia acuta. Fl. 7-9. Cor. violaceo purpurea. In agris plerumque inter segetes frequens.

\section{SEDUN'}

Cal. inf. 5-12-fidus. Cor. inf. 5-12-petala. Nect. squamae 5-12 ad basin germinum. Caps. 5-12 siliquiformes 1-locular. margine interiore se. miniferae et dehiscentes.

* Corolla pentapetala. (Sedum.)

+ Foliis planis. (Telephium.)

1. S. Telephium: caule erecto, fol, planis oblong. dentatis $\nabla$. serrato-deutatis, corymbo composito cymoso-folioso. S. I. 257. H, A. VI. 13.

A. 1-2, 4. Rad, tuberosa. Fl. 7, S. Cor。 viridicanti-lutescens. In agris siccioribus, coes meteriis etc. frequens.

$\uparrow \div$ Foliis teretibus. 
2. S. reflexum: fol. subulat. spars, glabr. basi solutis, inferiorib. recurvatis, flor. cymosis foliosis, petal. lanceol. acuminatis. S. I. 258. Kth, 124.

A. $\frac{5}{2}-1 \cdot 2$ f. Fl. 6, 7. Cor. Alava. In collibus aridis sylvarum. Grunw. Piclielsd. Spand. etc frequens.

3. S. acre: fol. subovat, circumsciss, gibb. erectiuscul. sparsis, cym. 2-partitis, sepal. circumsciss. gibbis. S.1.258. II. A. I. 15.

A. 1-2" 24. Fl. 5, 6. Cor. lutea, Sapor acris. In locis siccis undique. Off.

4. S. sexangulare: fol. subcylindraceis circum sciss. gibb. erectiuscul. 6-fariam imbricatis, cym. 3.partitis, sepal. circumnexis laevibus. S. I. 259. H A. I. 16.

A. praecedentis. 24. Fl, 6, 7. Cor, lutea. In locis siccis frequens.

5. S. villosum: fol. oblongis supra planiusculis erect. sparsis pedunculisque axillarib. subuniflor. pubescentibus, petal. ovat. obtusiusculis. Kth, 125. S. I. 259 .

A. $1-2^{\prime \prime} \odot$. Fl. 5. Cor. purpurea. In pratis paludosis prope Steglitz. Kth. S.

* Corolla dodecapetala (Sempervivum).

6. S. tectorum (Scopoli): fol. ciliatis, propaginibus patulis, flor. 12-gynis, nect, cuneiformib، carunculatis. S. I. 259. 
Serpervivum tectorum. L. IV. II. 932. H. A. I'I 14. A. $1-1 \frac{\pi}{2}$. Fl. 6, 7. Cor, purpurea. Nect. 12. alba. In muris et tectis linc inde. In Dörfern.

$$
\text { 203. OXALIS. }
$$

Cal. inf. 5-sepalus. Cor. inf. 5-petala, petal. unguibus connexis. Stam. inaequalia, filament. basi connatis, altern. minoribus. Caps. 5-rostris 5-locular. 5-valvis, valvis medio septiferis. Spermoph. in centrali angulo loculamentorum longitudinale. Sem. arillata.

1. O. Acetosella: scapo 1.floro fol. 3-natis longiore, foliol. obcordatis, styl. stanina longiora superantibus. H. A. V. 3 s.

A. 2-6" 24. Rad. repens. Fl. 4, 5. Cor. alba venis purpureis. In sylvis humidis frondosis. Papen b. Teg. Schönh. etc. Off.

3. O. stricta: caule plerumque erecto ramoso, fol. 3-natis, foliol. obcordatis, peduncul. umbelliferis, petal. integr, rotund.tis, styl. longitudine staminum longiorum. H. A. V. 40.

A. 2-8" 4 . Rad. repens. Fl. 5-9. Cor. citrina. In agris soli melioris frequens. 


\section{C O S A N D R I A. \\ ORDO I. MONOGYNIA, \\ 204. A S R U M.}

Cal. sup. 3-4-fidus persistens. Cor. o. Caps.calyce coronata 6-locul. evalv. polysperma, dissepiment. centro liberis. $S \mathrm{~cm}$. dissepimentis affixa. 1. A. europaeum: fol, reniformib. obtus, glabr. geminatis, cal. 3.fido. WV. Enum. I. 495. H. A. I. 44. S. I. 261.

A. 2-6"24. Caulis repens. Fl. 5. Cal. atropurpureus. Stam. 12. In sylvis frondosis. $\mathrm{Pa}$. penb. rarius. W. OfE.

205. IXTHRUM.

Cal. inf. 1-sepal. 6-12-dentatus, Cor. 6-petala, Caps. sup. 2-locul. 2-valv. polysperma, dissepiment. valvis contrario. Spermoph. centrale dissepimento adnatum.

1. L. Salicaria: fol. opposit. cordato.lanceolatis, Hor. spicat. 12-andris. L. W. II. 865 . H. A. III. 39. A. 1-4.24. FJ. $6-8$. Cor. purpurea. In locis humidis frequens.

2. L. Hyssopifolia: fol, alternis linearibus, florib. 6-andris. L. W. II. 869 .

A. 3-8" $\odot$. Fl. 6-8. Cor. rubro-violacea. In humidis graminosis rarius. Nach Strablau Lin. W. Heinersd, S." 
206. PPU $U$ U S .

Cal. inf. 5-fidus, Cor. inf. 5-petala. Drupa sup. nuce 1 , suturis prominulis.

* Floribus racemosis. (Padus.)

1. P. Padus: fol. deciduis duplicato-serrat. sub. rugosis, petiol. 2-glandulosis, racem. subpendulis, petal. integris, nuce scrobiculita. L. IV. II. 984. H. A. IV. 40 . S. I. 263 .

A. $25-30^{\circ}$ T. Fl. 4-5. Cor. alba. Drupa subcordato-rotunda nigra. In sylvis hnmidiusculis passim. Thierg. Teg. Friedrf. Off.

2. P. seratina: fol. deciduis subcoriaceis glabris simpliciter serratis, serraturis inferiorib. glanduliferis, petiol. raro glandulosis, racemis lax. petal. integris. II. F. 70. S. I. 263.

A. 30-40' h. Fl. 6. Cor. alba. Drupa sub. rotunda, nigra. Im Thierg. rarius. * Floribus umbellatis. (Cerasus.)

3. P. Cerasus: ram. pendulis, fol. ovat. utrinque acut. duplicato-serratis, juniorib. subtus pubescentibus, seniorib. glabris, petiol. eglandulosis, umbell. subsessilibus. H. A.IV. 42. S.I. 263.

A. Io-15' 五. Fl. 4, 5. Cor. alba. Drupa subglobosa, atro-sanguinea, acida. Ad agros es circa pagos culta. Uff.

4. $P$. avium: ram. patentib. rectis, fol. obovatis serrat. omnib. pubescentibus, petiolis plerumque 
2-glandulosis, umbellis subsessilibus. II. F. 72 . S. I. 264 .

A. $10-20^{\prime}$ 万. Fl. 4, 5. Cor. alba. Drupa subglobosa, sanguinea, dulcis. In sylvis frondosis passim. Falkenhagen S. Thierg. Off.

*** Fioribus subgeminatis. (Prunus.)

5. P. domestica: ramis inermibus, fol. lanceolatoovatis acuminat. serrat. subtus pubescentibus, petiol. eglandulosis, flor. pedunculat. singulis geminisve. H. A. IV. 42 . S. I. 264.

A. $15-20^{\circ}$ 色 Fl. 4, 5. Cor albida, Drupa atro-coerulea, ovoidea. Hinc inde circum pa. gos et hortos.

6. P. spinosa: ram. spinescentibus, fol. ovatolanceolat. acut. serratis, supra glabris, subtus pubescentibus, petiol. eglandulosis, flor. pedunculat. solitariis. H A. IV. 42. S. I. 264.

A. 2-8. T. Fl. 4 , 5. Cor. alba. Drupa oroideo.globosa, atro-coerulea. In fruticetis, collibus, circa pagos. Kalkb. Panck. Teg. etc.

\section{ORDO II. DIGYNIA.}

207. ACRIMONIA.

Cal. inf. 1-sepal. 5-dentat. persistens. Cor. inf. 5-petala. Achaen, 2 calyce indurato inclusa et fundo ejus adnata. 
1. A. Eupatoria: fol caulin pinnatis, foliol ob. longo-ovatis, spic. elevatis, petal. calyce duplo longioribus, calyce fructifero hispido. H. A. II.19. S. I. 265 .

A. 2-3' 24. Fl. 6, 7. Cor, lutea. In locis graminosis frequens.

\section{ORDO III. TRIGYNIA.}

203. RESEDA.

Cal. inf. 1-sepal. 4-6-partitus. Cor. inf. 4-6-petala, petal. laciniatis. Caps. sup. 1.locular. apice 3-dentat, dehiscens polysperma. Spermoph. 3. longitudinalia parietalia.

1. R. Luteola: fol. lanceol. integr. basi utrinque 1-dentatis, cal. 4.fidis. L. W. II. 876 .

A. I-3' $\odot$. Fl. 6, 7. Cor. lutescens. In ruderatis et coemeteriis hinc inde. Friedrf. Schöneb. Weilss.

\section{ORDO IV. PENTAGYNIA.}

209. Prinus.

Cal. sup. 5-fidus. Cor. 5-petala. Pomum calyce coronatum 3-5-loculare, loculis 2-spermis.

* Pomo triloculari, foliis pinnatis (Sorbus).

1. P. aucuparia (Mcench. Gärtner): fol. impari- 
pinnatis, pinnis subsimpliciter serratis supra subpubescentibus sublus et in petiolo communi villoso-prbescentibus, sem. in loculamentorum apice appensis. S. I. 266.

Sorbus aucuparia. H. A. IV. 45. H. F. 75.

A. $10-251$ 方. Fl. 5. Cor. alba. Pomum scarlatinum. In nemoribus et ad vias frequens. * Pomo 5-loculari, foliis simplicibus (Pyrus).

2. P. communis: fol. ovat. serrat. glabris, juniorib. subtus villoso-pubescentibus, cym. terminalib, foliosis. II. F. 84. S. I. 267.

๙. syluestris, B. domestica. A. $40-60^{\circ}$ 万. F]. 4, 5. Cor. alba. In sylvis frondosis et in agris hinc inde. Thierg.

3. P. Malus: fol. subrotundo-ovat. acuminat. serrat. glabrís, cym. terminalibus, ungue petalor. calyce breviore. H. A. IV. 46 .

Praecedenti similis iisdemque locis obvius. Off. 210. MIESPIIUS.

Cal. sup. 5-fidus, Cor. 5-petala. Bacca calyce coronata $2-5$-sperma.

* Foliis pinnatifidis lobatisve (Oxyacantha).

1. M. Oxyacantia (Willdenow): spinosa, fol. 3-lobis (rarius subpinnatificlo 5 -Lobis) glabris, lo- 
bis incis. inaequaliter serratis, cymis terminalibus, flor. plerumque 2-gynis, calycib. plerumque glabris, laciniis patentib, acutis. H. F. 81. S. 1. 267. Crataegus Oxyacantha L. W.

A. $8-10^{\prime}$ T. Fl. 5. Cor, alba. Baccae oblongo-rotundae, rubrae. In nemoribus ad sepes hinc inde. Jungfh. Thierg. etc.

2. M. monogyna (Willdenow): spinosa, fol. subpinnatifido-5-lobis (rarius 3-Iobis) glabris, lob. patul. apice incis, inacqualiter serratis, cym. terminalibus, flor. plerumque 1 -gynis, cal. plerunque pubescentibus, Iaciniis reflex, acuminatis. H. F. S1. S. J. 269.

Crataegus monogyna. L. IV. II. I0o6.

Anteced. semilis iisdemque locis obvia.

* Foliis integris (M espilus).

3. M. germanica: spinosa $v$. inermis, fol. oblongo-lanceolat. plerumque integerrim. subtus tomentosis, for, terminalib. sessilib. solitariis. H, F, 76 . S. I. 268 .

A. $s-12$, 卉. FI. 5, 6. Cor alba. In nemoribus et ad vias rarius. Teg. Kth.

211. SPIRAEA,

Cal. inf. 5-fidus. Cor.: 5-petala. Caps. 5. v. plures 1 -locular. 2-valv. oligo $v$. polyspermae. Sem. suturae interiori capsularum affixa. 


\section{* Caule fruticoso.}

1. S. salicifolia: fol. oblongo-lanceol. subacut. basi cuneiformib. undique serrat. glabris, racem. coarctat. composit, terminalibus. H, F. 89 .

A. 4-5 万 Fl. 6-10. Cor. pallide rubra, Hinc inde culta Thierg. Spand. Chaussee.

2. S. tomentosa: fol. ovato-oblong, acut. subtus tomentos. in costis et venis ramulisque ferrugineo-tomentosis, racem. terminalib. alaribusque compositis. H. F. 9o,

A. $3-4^{\prime}$ Cor. rubra. Culta rarius. Spandauer Chaussee.

$$
\text { * Caule herbaceo. }
$$

3. S. Filipendula: caule herbaceo, fol.interrupte pinnatis, pinnis uniformib. inciso-serrat. glabris, cym. terminalibus, rad.tuber ssa. S. I. 26.g. H. A. VII. 30 .

A. $1-2^{\prime}$ 4. Tl. 5-7. Cor. alba. In agris sylvis collibusque siccis graminosis. Jungfh. Papenb. etc.

4. S. Ulmaria: caule herbacec, fol. interrupte pinnat. subtus tomentosis, pinnis duplicato-servatis, cym. terminalib. supradecompositis, rad. lignescente fibrosa. S. I. :69. H. A. VII. 31.

A. $1-5^{\prime} 4$. Fl. 5-8. Cirr. albida. In locis luumidis copiosissima. 


\section{ORDO V. POLYGYNIA.}

212. ROSA.

Cal. sup. 5-partitus. Cor. 5-petala, Achaen. plura hispida a receptaculo urceolato intus hirto car. noso calyce coronato inclusa.

1. $R$. villosa: cal. subglobosis, peduncul. hispidis, foliol. utrinque tomentos, subtus glandulosis. S. I. 270 ,

a. vulgaris, $\beta$. mollissima. A. 4-6, 下. Fl. 6. Cor. rosea. In fruticetis ad vias etc. Jungfh. Teg. $\beta$, Grunw. Kalkb.

2 R. rubiginosa: cal. ovatis, petiol. glandulosohispid. aculeatis, foliol. ovat. subglabr. subtus glanduloso-pubescentibus. S. I. 271.

$\boldsymbol{\alpha}$ vulgaris, $\beta$. triflora, $\boldsymbol{\gamma}$. parvifolia, \$. umbellata, slabra. A. 5-6, 7 . Fl. 6. Cor. rosea. Ad sepes in collibus etc. frequens.

3. $R$. canina: cal. oyat. pedunculisque glabris, foliol. glabris subtus glaucescentibus, serratur. petiolisque subglandulosis S. I. 272.

a. vulgaris, f. glandulosa, $\boldsymbol{\gamma}$. pubescens. A. 5-6. 万. F1.6. Cor. rosea. In locis antecedentium copiosa.

4. R. sepium (Borkhausen): cal. ovat. pedunculisque glabris, foliol, pubescentibus, petiol. villo. sis S. I. 273.

a. hirsuta, $\beta$. laevigata. In fruticetis et nemoribus passim. $\alpha$, Schöneb. Kalkb. $\beta$, Kalkb. S. $1,1$.

F. B. 
213. RUBUS.

Cal. inf, 5-fidus, Cor. 5-petala. Bacca e baccu. lis 1-spermis composita.

1. $R$. Idaeus: caule aculeis rect. setoso-subulatis armato $v$. inermi, fol. quinato-pinnatis $v$. ternatis, foliol. breviter acuminat. subtus tomentosis, petiolis canaliculatis. H. A, III. 8. H. F. 105.

A. 3-6' h: F1. 5-6. Cor. alba Baccae rubrae. In sylvis humidiusculis et humidis frequens. Thierg. Teg. Jungfh. etc Off.

2. $R$. caesius: caule tereti caesio-pruinoso, aculeis parum recurvatis, fol. ternat. pubescentibus, cal. glanduloso-pubescente, petal. obovat. emarginatis. H. F. 107. H. A. III. 9.

Canlis prostratus h. Fl. 6-9. Cor. alba. Baccae nigrae pruina caesia. In agris undique.

3. $R$. nemorosus (Hayne): caule tereti villoso, aculeis subrectis, fol. villosis, caulium sterilium quinatis, fertilium ternatis, cal. glanduloso pubescente, petal. ovat. integerrim. v. emarginatis, $\mathbf{H}$. F. 107. H. A. IIJ. 10:

A. $1-1 \frac{1}{2}$ h. Fl. 6 ; 7. Cor. rosea, ravius alba. Baccae nigrae. In fruticetis et ad sylrarum margines hinc inde. Spand. Heide. S. Teg. Thierg.

4. R. corylifolius (Smith): caule 5-gulari subglaxo, acule is recurvis, fol, quinat. ternat. simplici- 
busque villozis, cal. subglabro, fructifero reflexo, petal, obovat. integerrimis. H. F. 108. H. A. IIJ. 11 . A. $2-4^{\prime}$ ' . Fl. $6-9$. Cor.rosea, rarius alba. Bac. cae nigrae. In fruticetis ad sepes etc. undique. 5. R. fruticosus: canle 5-angulari subtomentoso, aculeis recurvis, fol. quinat. ten nat. simplicibusve superne glabr. subtus tomentosis, cal. subtomen: ioso fructifero reflexo, petal. obovat. integerrimis; H. F. 109. H. A. III, 12.

Antecedenti similis h. In collibus, frutice. tis, sylvaticis passim. Kalkb. Chamisso. S. I. 275.

6. S. saxatilis: caule herbaceo stolonifero erecto anguloso pubescente, aculeis tenuib. r'ct', patentibus, fol. ternatis, peduncul. 3-5 floris. H. F. 110. A. $\frac{T}{2}-1^{\prime}$ 2. Fl. 6, 7: Cor albida. Baccae rubrae. In sylvis hinc inde. Bamb. S. Thierg. VV. Jungfh. 'Teg.

$$
\text { 214. FRAGARIA. }
$$

Cal. inf. 10-fidus. Cor. 5-petala. Achaen. carpo. phoro carnoso deciduo inserta.

1. F. semperflorens (Hayne) : laciniis calycis frtı ctiferi pilisque petioloxum divergentibus, pedunculorum patentibus, pedicellorum adpressis, H. A. IV. 25.

Caulis sarmentosus. 2\%. Fol. ternata. Cor. alba. Receptac. conoidenm. In sylvis umbrosis frequens. Jungfh. Giunw. Teg etc. 2. F, vesca: laciniis calycis fructiferi rellexis, Mi 2 
pil. petiolor. divergentibus, pedunculorum patenti. bus, pedicellorum adpressis. H. A. IV. 26.

Caulis sarmentosus. 24. Fol. ternata. Fl. 5, 6. Cor. alba. Receptac. subglobosum. In sylvis et nemoribus undique.

3. F. collina: laciniis cal. fructiferi erect. adpressis, pilis petiolorum divergentibus, pedunculorum plerumque divergentibus, pedicellorum plerum. que patentibus. H, A, IV. 30.

Antecedentibus similis 24. Fl. 5, 6. Receptac. oblongo-subrotundum. Habitus partium major. In collibus graminosis hinc inde. Kalkb. Willmersd.

215. POTENTIILA.

Cal. 8-ro.fidus. Cor. 4-5-petala. Achaen. subdrupacea numerosa receptaculo parvo conico exsucco inserta.

* Calyce 8-fido, Corolla 4-petala (Tormentilla).

1. P. Tormentilla (Schranck): caule adscendente dichotomo, fol. ternatis, caulin. sessilibus, foliol. lanceolato-ovalib. inciso-serratis, pedicell, solitar. dichotomalib. lateralibusque, cor. sub-4-petalis, petal. obcordatis. Lehm. Pot. 149.

Tormentilla erecta. H. A. II. 48. Kth. 143.

A. $\frac{1}{2}-1 \cdot 24$. Rad. lignosa. crassa. Fl. $6-9$. Cor. lutea. In sylvis subhumidis et circa pi.ludes frequens. OI. 
2. $P$. nemoralis (Nestler': caule prostrato filifor. mi, fol. quinat ternatisque subpedatis, foliol. obo. vat. basi cuneatis, superne inciso-serratis, subtus adpresso-pilosis, pedicell. axillarib. solitariis elongatis, flor. sub-4-petalis, petal. obcordatis. Lehm. Pot. 147.

Tormentilla reptans. Kth. 143.

F1. 6, 7, 8. 4 Cor. Hava, interdum 5-petala. In sylvis rarius. Thierg. W. Teg.

* Calyce 10-fido, corolla 5-petala (Potentilla).

+ Foliis pinnatis.

3. $P$. rupestris: caule erecto dichotomo, fol. ra. dicalib. pinnatis, caulin. ternatis, foliol, subro. tund. basi cuneat, serrato-dentatis, stipul. indivi. sis. Lehm. Pot. 47.

A. $\frac{3}{4}-x^{\prime} 24$. Fad. cylindrica. Fl, 6, 7. Cor. alba. In locis herbidis sylvarum. Briselang rarius. Meyer.

Pauca hujus Potentillae specimina reperta sunt ab A. Meyero, cui nostrum quoque debemus. 4. $P$. supina: caule decumbente dichotomo, fol. pinnatis, foliol. oblong. inciso-scrratis, pedicell. axillarib. solitariis. Lehm, Pot. 42.

A. $\frac{1}{2}-I^{\prime} \odot$. Pad. fibrosa. F1, $6-8$. Cor。 lutea parva. In ruderatis pagorum et ad ripas aquarum. Vor d. Strabl. Th, Weifs. Friedrf.S. 
5. P. anserina: caule ropente, fol interrupte pinnatis, foliol argute serrat subtus sericeis, pedicell, axillarib. solitar. longitudine foliorum, stipul. multifidis. I.chm. Pot. 7 1. H. A. IV. 31.

Rad. fibrosa 24. Cor. magna, Alava. In locis subhumidis et siccioribus, praescrtim prope pagos, vulgaris.

$$
\div+ \text { Folits rigitatis. }
$$

6. P. alla: canle procumbente debili paucifioro, fol. inferiorib. quinatis, superiorib. ternatis, foliol. oblong, apice conniventi-serratis, supra gla. bris, subtus sericeo-cancscentibus, petal, obcordat. calycem superantibus Lelim. Pot. 132.

A. 3-5" 24. Rad. lignosa. F1. 5. Cor alba, magna. In sylvis frondosis subapertis. Jungfh. Papenb. S. Thierg.

7. P. argentea: caule adscendente, fol. quinatis, foliol. cunciformibus, superne incisis, margine revolutis, subtus candido-tomentosis, petal. retus. calyce paullo longioribus. Lehm. Yot. 94.

A. 4-12"24. Rad. sublignosa. Fl. 6-9. Cor. Iutea. In solo sicciore, ad vias, in agris etc. frequens.

8. $\boldsymbol{P}$. subacaulis: caule decumbente, fol. quinatis ternatisve, foliol. obovat. rotundato-obtus. crenatis, pilis adpress. stellat. subincano-lomentosis, 
petal. obcordat. calyce fere duplo longioribus. Lehm. Fot. 120.

Potentilla verna. WW. p. n. 347. et Kth. 142.

A. 2-8" 2. Rad. sublignosa. Fl. 5. Cor litea. Planta, quod ad foliorum formam et inflorescentiam, admodum variabilis, ita ut pili stellati soli certam constituant notam. In agris et sylvis arenosis copiosissima.

9. $P$. verna: caule declinato, fol. infim. quina tis, foliol. obovato-cuneiformib. superne profunde serrat. subretus. utrinque pilosis, petal- obcardat. cal. longioribus. Lehm. Pot, $10 \%$.

In sylvis et collibus praecedente, cui sis millima, rarior. Kalkb. S. Jungth. Grunw.

10. P. opaça: caule decumbente filiformi, fol. infim. septenat. quinatisque, foliol. lanceolato-cum neiformib. profunde-serrat. utrinque prolixe pilosis, petal, obcordat, calycem aequantibus. Lehm. Pot. 105.

A. 2-8" 24. Fl. o, 7. Caulis saepe purpus rascens. Cor. lutea. In sylvis et inter frutices frequens. Grunw. Thierg. etc.

11. P. reptans: caule sarmentoso, fol. quinatis, foliol. obovato-cuneiformib. argute serrat. pilosis, pedicell. axillarib. solitar. foliis longioribus, pe. tal. obcordat. calyce longioribus. Lehm. Pot. 1440 S. I. 282 .

F1. $6-8 " 2$. Cor. magna, lutea. In sylris 
umbrosis et locis subhumidis graminosis frequens.

\section{$t+\dagger$ Foliis ternatis.}

12. P. norwegica: caule erecto superne dichotomo, fol. ternat. petiolatis, foliol. oblong. acute serrat. patenti-pilosis, pedicell. axillaribus, petal. obovat, calyce brevioribus. Lehm. Pot. 153.

A. 4-12" $\odot$. Cor. lutea. In humidiusculis graminosis circum lacus et paludes sylva. rum camporumque rarius. Jungfh. Beyrich. Weirs. S. I. 283 .

\section{6. $\cos$ A $\mathrm{R}$ M.}

Cal. colorat. 10-fidus. Cor. 5-petala, petal. calyce minoribus, Achaen. subdrupacea receptac. ovato basi attenuato spongioso persistenti inserta. C. palustre. L. W. II. 1119. Kth. 145. Potentilla palustris. Lehm. Pot. 52. S. I. 277.

A. 1-1 $\frac{r^{\prime}}{2}$ 4. Rad. repens. Caul. adscendens. Fol. pinnata. Fl. 5-8. Cal. atro-purpureus. Cor. atro.purpurea. In locis uliginosis, Thierg. Jungfh, etc. frequens.

\section{OEUM.}

Cal. inf, 10-fidus, Cor. 5-petala. Achaen. pluri. ma stylo geniculato aristata receptaculo columnari imposita.

1. G. urbanum: fol, radicalib. interrupte lyrato.pinnatis, caulin, ternat. trilobisque, flor. erectis, 
cal. corolla longioribus, aristis glabris, primum inflexo-geniculatis, dein uncinatis. S. J. 284 . H. A. IV. 33 .

A. $1-2^{\prime}$ 24. Rad. praemorsa subperpendicularis. Cor. lutea, parra. Frequens in umbro. sis, in nemoribus, fruticetis. etc. Off.

2. G. rivale: fol. radicalib. interrupte lyratopinnatis, caulin. plerumque trifidis, for. nutantibus, cal. corollam aequantibus, arist. primum gyroso-geniculat. plumosis, dein uncinatis. S.I. 285 . H. A. IV. 34:

A. $\frac{1}{2}-2 \cdot 4$. Rad. subhorizontalis. FI. 5, 6 . Cor. lutescenti-brunneo-purpurascens. In pratis locisque humidis frequens.

3. G. intermedium (Ehrhardt): fol, radicalib. interrupte pinnatis, caulin. ternat. trilobisque, Hor. cernuis, cal. petalis longiore, arist. primum in. Hexo-geniculat. plumosis, dein uncinatis. S. I. 145. Rad. et fol. radicalia $n t$ in $G$. rivali, fol. caulina ut in G. urbano. Cal. G.rivalis. Cor. lutea fere ut in G. urbano. Receptac. et arista. G. urbano similis. In sylvis humidis ra. rius. Thierg. 


\section{Cl. XII. POLYANDRIA. \\ ORDO I. MONOGYNIA.}

218. CIIEIIDONIUI.

Cal. inf. 2-sepal. deciduns. Cor. 4-petala. Caps. sup. siliquiform. 1-locul, bivalvis. Spermoph. suturale. Sem. crista glandulosa instructa.

1. C. majus: fol. decursive pinnfis, pinn. sublobat. crenatis, flor. umbellatis, petal. integris. S. I. 256 . HI. A. IV. 6.

A. $1-3^{\prime} 24$. Fl. 5-0. Cor. Intea. Herba tota croceollactescens. In nemoribus ad pagos etc. frequens. Off.

219. PAPAVER.

Cal. inf. 2-sepal. deciduus. Cor. 4-petala. Caps. sup. suburceolata sub stigm. persistente sessili plerumque poris dehiscens. Spermoph. Iongitudinalia paríetalia.

- Capsulis toris hispidis (Argemone).

1. P. Argemone: caule folioso hirto multifloro, fol. bipinnatifidis, caps. clavato-urceolat. hispidis, pil. caulis pedunculorumque adpressis. S. I. 287. II. A. VI. 37.

A. $\frac{\pi}{2}-1^{\prime} \odot$. Fl. 6-8. Cor, cinnabarina. In agris hinc inde obvium. 
- Capsulis glabris v. apice parce pilosis (Rhoeas).

2. $P$. dubium: canle folioso hirsuto multifloro, fol. pinnatifidis, laciniis incisis, caps. oblongourceolat. glabris, pilis caulis divergentibus, pedunculorum adpressis. S. J. 257. H. A. VI. 38.

A. $\frac{\pi}{2}-1 \frac{I^{\prime}}{2} \odot$. Fl, $6-9^{\prime}$ Cor. cinnabarina. Inter segetes, in arvis copiosissime.

3. P. Rhoeas: canle folioso hirsuto multifloro, fol. pinnatifidis, laciniis incisis, caps, obovato-ur. ceolat. glabris, pil. caulis pedunculorumque divergentibus. S. I. 287. II. A. VI. 38.

Antecedenti similic. A. $\frac{\mathrm{I}}{2}-1 \frac{\mathrm{r}}{2}{ }^{\prime} \odot$. Copiosis. sime inter segetes. Off.

4.P. somniferum: canle folioso glabro multilloro, fol. amplexicaulib. incisis, caps, ovato v. subgloboso-urceolat. glabris, pil. pedunculorum divergentibus. S. I. 287. H. A. VI. 40.

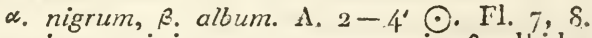
Cor. in $\alpha$. miniato-purjurascens, in $\beta$. albido. violascens. Crebro in agris cultum. Off.

$$
\text { 220. NYMPHAEA. }
$$

Cal. 4-5-sepal, marginem receptaculi cingens. Cor. polypetala receptaculo inserta. Nect. glandula in medio stigmate. Bacca spuria multilocul. (caps, plurimae a receptac. inclusae et 
cum eo connatae) locul. polyspermis, sem. arillatis.

1. $N$. albu: fol. cordat. integerrimis, stigm. 16-20-radiato, radiis adscendentibus. S. I. 288. H. A. IV. 35 .

. major, $\beta$. minor. Rad. crassa horizontalis. 24. Petiolorum vasa aërophora quatuor media maxima. Fl. 6, 7. Cor. alba. In fossis et lacubus $\alpha$. frequens, $\beta$. in paludibus rarior.

221. NENUPHAR.

Cal. 5-6-sepal. imo receptaculo insertus. Cor. polypetala receptaculo inserta. Nect. dorsum petalorum. Barca spuria muliilocul. (caps. plurimae intra receptaculum quocum connatae ab. sconditae) locul. polyspermis, sem. non arillatis. 1. N. lutea (Hayne): cal. 5-sepalo, stigm. repando 14-20-radiato profunde umbilicato, bacca lae$\mathbf{v i}$, fol. ovali-cordatis, lobis approximatis, petiol. apice triquetris H. A. IV. 36.

Rad. horizontalis crassa 2 . Petiolorum vasa ärophora aequalia. Fl. 6,7. Cor, vitellina. In fossis et lacubus frequens.

\section{TIIIA.}

Cal. inf. 5-partitus. Cor. 5-petala. Nect. corona 5-petala v. o. Caps. drupacea basi 5-valv. 5-lo. cularis, locul. 4-abortivis 2-sperma. 
1. T. parvifolia (Hoffmann): fol. subdimidiatocordat. glabris, subtus venarum axillis barbatis, petiol. foliorum superiorum dimidio folio longiori. bus, nectar. o., peduncul. multifloris, fructu laevi obliquo. H. F. 111. H A. III. 46.

A. $40-60^{\circ}$ 万. Fol. subtus pallide glancescentia, barbis ferrugineis. Fl. 7. Cor. ex albo et Havescenti-viridis. Ad vias et in nemoribus frequens.

2. $T$. vulgaris (Hayne): fol. subdimidiato-cordat. glabris, axillis venarum subtus barbatis, petiol. superiorum foliorum dimidio folio brevioribus, nectar. $o$, peduncul. multifloris, fructu sublaevi regulari. H. F. 112. H. A. III. 47.

A. $40-6 o^{\prime}$ 万. Fol. concoloria, laete viridia, barbis fuscescentibus. Fl. 6, 7. Cor. pallide citrina. In nemoribus et ad vias frequens.

3. T. pauciflora (Hayne): fol. subdimidiato cor. dat. villoso-pubescentibus, axillis venarum inferia. rum subbarbatis, nectar. o, peduncul. 3-5-floris, fructu costato. H. F. 112. H. A. III. 48 .

A. $40-60^{\prime}$ h. Fol. multo majora, grosse serrata, barbulis albidis. Fl.6. Cor. magna, pallide citrina. Ad vias et in pagis frequens.

223. HELIANTMEMUM.

Cal. inf. 5-sepalus, sepal. 2 minoribus. Cor.5-pe. 
tala. Caps. 1-locul 3-valv. polysperma. Sem. spermoph. linearib, valvularibus affixa.

1. H. vulgare (Gaertner): caule frutescente prostrato stipulato, fol. oblong. margine revolut. subpilosis, stipulis lanceolatis, racemis terminalibus vagis $H . F .114$.

Cistus Helianthemum. L. IV. II. 1209.

A. $\frac{x}{2}-1^{\prime}$ t. Fl. 5, 8. Cor. lutea. In pratis siccioribus et pascuis satis frequens. Friedrf. Teg. Schonh. etc.

\section{ORDO II. TRIGYNIA.}

22\%. EUPHORBIA.

Cal. 1-sepal. ventricosus. Cor. 4 v. 5-petala, calyci inserta. Gernt. pedicellatum. Caps. tricocca, coccis 2.valr. 1-spermis.

* Umbella triradiata.

1. E. Peplus: umbella trifida; dichotoma, invo. lucell. ovatis, fol. integerrim. obovat. petiolatis. L. W. II. 903.

A. 3-12" ค Planta tota glabra. Fl. 7,8 . Cor. Hivo-viridicans. In pagis ruderatis, agris etc. frequens.

2. E. exigua: umbella trifida; dichotoma, involucell, lanceolatis, fol. linearibus, L. W. II. 903. A. 3-6" ○. Fl. 6, 8. Cor, lavo-viridicans, 
In agris argillosis inter segetes: $B$. Panck, $u$. Schönh. Rose. Schulzend. WValter. S. I. 292. * Umbella quinqueradiata.

3. E. helioscopia: umbella 5.fida; bifida, dichoroma, involucell. obovatis, fol. cuneiformib. ser. rat. glabris, caps. laevibus. L. W. II: 914. II, A. II. 20 .

A. 3-8' . Canl. patentim pilosus. Fl.6-9. Cor. flavo-viridicans, petal. truncatis. In ruderatis, agris, ad pagos etc. frequens.

$$
\text { * * Umbella multiradiata. }
$$

4. E. Cyparissias: umbella multifida; dichotoma, involucell. subcordatis, petal. lunatis, ram. sterilibus, fol. linearibus, caulin. lineari-lanceolatis. II. A. II. 22 .

A. $\frac{\pi}{2}-1 \frac{\pi}{2}{ }^{\prime} 24$. Rad, subrepens. Involucr, sub. reflexum. Fl. 5-7. Cor. Havo-viridicans. In agris arenosis undique.

5. E. Esula: umbella multifida; dichotoma, involucell. subcordatis, petal. subbicornibus, ram. sterilibus, fol. lanceolatis، S.I. 29 f. H. A. II.24.

Euphorbia segetalis. Kth. 152 .

A. $\frac{r}{2}-2 \frac{1}{2} \cdot 24$. Fol. basi valde attenuata. FI. 5-9. Involucr. plerumque patens. Cor. antecedentis. In solo meliore. Vor d. Hallischen Thor. Weilss. etc.

6. E. palustris: umbella multifida; subtrifida, bifida, involucell, ovatis, petal. truncat, subpeltatis, 
ramis sterilibus, fol. lanceolatis. S. I. 294. H. A. II. 23 .

A. 3-5' 4. Rad. crassa, ramosa. Fl. 5-6. Cor. Havo-viridicans In pratis humidis locis. que uliginosis. Hinter d. Briselang.

225. DELPIN INIUNI.

Cal. o. Cor, inf. 5-petala, petalo superiore calcarato. Nect. 1-4-phyllum postice in calcar productum. Caps. $1-5$ valv. 1-loculares, margine interno seminifero.

1. D. Consolida: caule erecto glabro divaricatimramoso, flor. laxe racemosis, pedicell. bractea longioribus, nectar. I-phyllo, caps. unica glabra.

A. $\frac{3}{4}-1 \frac{1}{2} \cdot \odot$. Fol. tripartita lobis, trifidis. Fl. 6-9. Cor, violacea. In agris undique.

\section{ORDO III. PENTAGYNIA.}

226. A QUIIEGIA.

Cal. o. Cor. 5-petala, Nect. corona 5-phylla intra petala, foliolis calcaratis. Caps. 5 distinctae 1-locul. margine interiore superne dehiscentes. Sem, margini interno capsulae affixa. 1. A. vulgaris: calcarib. incurvis, caps. villosis, caule folioso multifloro foliisque glabriusculis, styl. stamina non superantibus. DC. S. I. 334. H. A. III. 6 . 
A. $2-3^{\prime}$ 4. Fol. ternata, foliol ternat. $\nabla$. trifidis. Cor. violacea, magna. In nemoribus subhumidis rarius. Friedrf. W. Schönh. Cöp. nicker Heide.

227. NIGEIIA.

Cal. o. Cor. 5-petala. Nect. 5, 8 v. plura 2-labiata 2-glandulosa. Caps. 5-10 connatae 1-2-locul. margine interiore superne deliscentes. Sper. moph. longitudinaliter angulo interno capsu. larum affixum.

1. N. arvensis: anther. apiculatis, styl. 5-7 extus circinnatim revolutis, caps. laevib. in fructum obconicum basi angustatum ulira medium conneis, caule glabro, ramis subdivergentibus. DC. S. I. 329. II. A. VI. 17 .

A. $\frac{\pi}{4}-\frac{r}{2} \cdot$. Fol, pinnata, foliol. linearibus. Fl. 7, s. Cor. coerulea. Nectar. ex cyaneo et lutescente varia. In agris inter segetes $v$. in incultis rarius. Schönh. W. Hinter Spand. S. I. 296 .

\section{ORDO IV. POLYGYNIA.}

228. THAXICTRUM.

Cal. 0. Cor. 4-5-petala. Achaen. plurima ecaudata receptaculo minuto imposita. 1. $T$. minus: caule tereti, flor paniculat, cernuis F. B. 
4-sepalis, folior. segment. glabr. apice dentat, sub. tus glaucis, achaen, ovat, utrinque acutis. S.I. 296 . a. collinum $\frac{\mathrm{T}}{2}-1 \frac{\pi}{2}^{\circ}$. $\beta$. dumosum $2-4^{\prime} 2$. Fol. glaucescentia. Fl. 6, 7. Cor. Havescens. In In agris, sepibus et collibus apricis frequens. Friedrf. Clarlottb. Kalkb, etc.

2. T. flavum: caule erecto ramoso sulcato, paur. erecta multiplici subcorymbosa, folior. omnium segment. cuneiformib. trifid. acutis, achaen. oblique cylindraceo-ovat. striatis. S. I. 297.

$\alpha$. pratense. $\beta$. sylvestre. A, $1-3 \cdot 4$. Rad. repens. Fol. laete viridia. Cor. Hava. Fl.6, 7 . In pratis et sylvis humidis frequens.

\section{PULSATILIA.}

Cal. o. Cor. 6-petala. Achaen. plurima recept. cylindrico imposita caudata, cauda plumosa.

1. P. vernalis: fol. quinato-pinnatis ternatisque, foliol. lateralib. bi- v. trifidis, medio trifido, laciniis oblong. inciso-dentat. pilosiusculis, flore erecto patente, involucro villoso.

๙. pinnatifolia. $\beta$. mixtifolia. $\gamma$. ternatifolia. A. $\frac{1}{4}-3^{\prime} \cdot 4$. Pad, multiceps. Fl. 3, 4. Cor extus villosa, alba striis purpurascenti-lilacinis v. lilacino-purpurea r. violacea. In sylvis acerosis hinc inde. Müggelsberge. Kll. Jungfl. (rarissime). Bctcke. Ercknersche Heide. S.

Magna speciminum e nostra regione lecta. rum copia comparata ad eam sumus adducti 
sententiam, $A$. patentem nostrae Florae incolam esse non posse eamque plantam, quan plurimi nostrae urbis Botanophili pro $A$. patente habuerunt, nullam aliam esse nisi eam varie. tatem $P$. vernalis, quam sub $\gamma$ comprehendimus. $A b$ initio quidem, in en haesitavimus, quod in $\gamma$ folia antecedentis anni semper emar. cida essent, quum in varietate $\alpha$ et $\hat{\sigma}$ viridia conspicerentur; sed seriore tempore specimina ab amic. Starckloffio accepta transitum probarunt. Pari modo in involucri villositate nullam invenire possmus differentiam.

2. P. vulgaris: fol. pinnatis, foliol. multipartitis, Iaciniis lanceolato-linearibus bifidis trifidisque, to. re suberec:o, petal. 6 rectis patentibiss.

Anemone Pulsatilla. S.I. 29: H. A. I.22.

A. 2-6"24. Rad. multiceps. F1, 3, 4. Cor. violacea. In sylvis et agris arenosis rarissima. Jungfl, an einer Sielle. Mr.

3. $P$. pratensis: fol, pinnatis, foliol. multipartiis, laciniis linearibus plerumque integris, Hore mutante, petal. 6 apice reflexis.

Anemone praicusis. S. I. 299. H. A. I. 23.

A. $\frac{\mathrm{r}}{2}-1 \cdot 2$. Rad. mulciceps. Fl. 3, 4. Cor. nigricanti-violacea. In agris et sylvis arenosis satis frequens. Jungfir. Grunw. els.

230. ANEMONE.

Cal. o. Cor. 6-9-petala. Achaen. plura ecaudsta. * Involucri foliolis petiolatis (Anenwone).

$N_{2}$ 
1. A. sylvestris: fol. radicalib. ternatis et quinatis, foliol. apice inciso-dentatis, involucralib. petiolat. conformibus, petal. 6 ellipticis, achaen. lanuginosis.

A. 1-2' 4. Rad. fibroso-fasciculata. Fl. 5,6. Cor. magna, alba $r$. purpurascens. In siccis nemoribus et collibus fruticosis hinc inde. Jungfl. Rahnsd. Kth. Kalkb.

2. A. nemorosa: fol. radicalib. ternatis, foliolis trifid. inciso-dentatis, involucralib. petiolat. conformibus, petalis plerumque ellipticis. H. A. I. 24. A. $\frac{r}{4}-\frac{r}{2}, 24$. Rad. cylindrica. Fl. 4, 5. Cor. alba interdum rubella. In humidis nemoribus frequentissima.

3. A. ranunculoides: fol. radicalib, ternatis et quinatis, foliol. subtrilidis inciso-dentatis, involucralib. breviter petiolat. inciso-dentatis, petal. 6 ellipticis. A. $\frac{1}{4}-\frac{I}{2}^{\prime}$ 24. Rad cylindrica. Fl. 4, 5. Cor. vitellina. In humidis nemoribus antecedente rarior. Blankenfelde. IV. Thierg. Kth. Papenb. Kalkb.S. Schönhausen.

* Involucri foliolis sessilibus calyciformibus. (Hepatica).

4. A. Hepatica: fol. trilobis, lobis integerrimis. H, A. I. 21 . S. I. 301 .

Hepatica triloba. DC. S.I. 216. Kth. 150.

A. 3-4" 24. Rad. fibrosa. Fl. 4. Flores prius quam folia nova erumpunt. Cor. 6 - petala cyaneo-violacea, Germina basi involucro ca- 
pillaceo dissecto cincta. Achaen. longe acumi. nata. In nemoribus frondosis et inter frutices frequens. Briselang. Papenb. Kalkb. S. Schön. hausen.

\section{I. $\triangle$ DONIS.}

Cal. 5-sepalus. Cor.5-15-petala, petal, ungue nudis. Achaen. plurima stylo persistente terminata receptaculo cy lindrico imposita.

3. A. flava (De Candolle): cal. gIabro basi solu. to, petal. plan. oblong. obtusis calyce duplo lon. gioribus, achaen. reticnlat. in capitulum oblon. gum aggregatis, caule simplici v, ramoso. DC. S. I. 222 . S. I. 302 。

a. pallida. $\beta$. miniata. A. $\frac{1}{2}-2^{\prime} \odot$. Fol. bi $\nabla$. tripinnatifida, laciniis linearib. acutis. Fl. 5,6 . Cor. pallide lutea $v$. miniata. In solo argillaceo inter segetes. Charlottb. S.

232. RANUNCULUS,

Cal. 3-8-sepalus. Cor. 5-polypetala, petal, ungue poro v. squana nectarifera instructis. Achaen. stylo persistente terminata receptaculo cylin. drico imposita.

Corollis albis, achaeniis transversim rugulosis (Batrachium).

1. $R$. aquatilis (Allioni): caule natante, in exsiccatis repente, fol, emersis tripartitis, immers, mul- 
tifidis, petal. obovat. calyce majoribus, achaen. setis rigid. hispidis, S. I. 303.

Ranisnculus heterophyllus. W. p. n. 590.

a. diversifolius. B. fissifolius. $\gamma$. capillifolius. $\delta$ rigidifolius. $S$. Planta polymorpha 24 . Cor. alba ungue luteo. In aquis et paludibus undique.

2. R. peucedanifolius (Allioni); caule natante, in radis repente, fol. omnib. submersis di- ant trichotome repetito-dissectis, laciniis longissim. linearib. parallelis, petal, calyce majoribus, achaen, glabris. S, I. zoi.

Ranunculus fluviatilis. W. p. n. 592. Kth. 155. Artecedenti similis, sed rarior. Teg. Kth. - Schaafgraben.

* Corollis luteis, foliis integris.

3. R. Myosurus (Afzelius): fol. omnib. radicalib. linearib. integerrimis, scapis folia dein superantibus, sepat. basi soluiis, receptac. Iongissimo, achaen. sriquetr. confertissimis. S. 1. 304.

Myosurus minimus. L. Kth. 89 .

A. 2-4" $\odot$. Rad. fibrosa. F1. 5, 6. Petala 4. parya Alarescentia. Stam. 5-20. In agris argillaceis frequens.

4. R. Flammula: fol, glabr. linearib. subovatisvo subintegris, inferiorib, petiolatis, caule erecto aut 
declinato farcto subradicante, peduncul. oppositifoliis, achien. laevibus. S. J. 305.

$\alpha$. hetcrop'yllus. $\beta$. lancifolius. $\boldsymbol{\gamma}$. declinatus. A. $\frac{x}{2}-1 \frac{\pi}{2} \cdot 24$. T1. 5-8. Cor. lutea. In palu. dibus, pratis etc. frequens.

5. R. Lingua: fol. infim. oratis, caulin. lanceo. lat. subserrat. sessilib. semiamplexicaulibus, caule arecto superne pubescente. S. 1.30.5.

๙. vulgaris. $\hat{\beta}$. gracilis. Rad. stolonifera. A. $1-3^{\circ} 2$. Fl. $5,6,7$. Cor. magna lutea. In paludibus, fossis etc. frequens.

6. R. Ficaria: fol. petiolat, subrotundo-cordat. subangulatis, caule adscendente glabro, peduncul. axillarib. terminalibusque. S. I. 306. H, A, V. 27.

Ficaria ranunculoides. DC. S. I. 304.

A. $\frac{r}{2}-1 \frac{r}{2}$. Rad. tuberosa, tuberibus elongatis. 24 . Fl. 4, 5. Cal. plerumque 3-sepalus. Cor. Alava. In solo humido sylvarum et inter frutices frequens. Thierg. Panckow etc.

* * Corollis luteis, foliis lobatis multifidis, achacniis laevibus.

7: R. auricomus: fol. glabris, radicalib. petiolat. cordat. plerisque tripartit. lobatisve, caulin. in lo. bos lineares integros subdentatosve partitis, calyco pubescente petalis breviore. S. I. 306.

A. $\frac{r}{4}-\frac{r}{2} \cdot 24$. Rad. fibrosa. Fl. 4, 5. Cor. Alava. In humidis sylvarum locis. Teg.Schön. haus. Thierg. etc. 
8. R. sceleratus: fol. glabris, radicalib. petiolat. tripartitis, lob. oblongo-linearib. integris, floralib. oblongis, cal. glabro, achaen. minimis in spicam elongatam dispositis. S. I. 307.

๙. vulgaris. $\beta$. minimus. A. $2 "-1 ' \odot$. Fl. 5,6. Cor. Aavescens parva. In paludosis, fos. sis, ad aquarum ripas etc. frequens.

9. $R$. repens: fol tripinnatis, pinnis cuneat. trilobat. inciso-dentatis, caule llagella prostrato-repentia e collo agente, florifero suberecto, cal. ad. presso, achaen. acumine recto. S. I. 307.

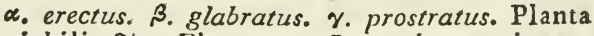
variabilis 24. Fl. 5-9. In agris, pratis etc. subhumidis frequentissima.

10. R. polyanthemus: fol. 3-5-partitis, partitionib. lobat dentat, aut inciso-multifidis, caule erecto petiolisque patentim pilosis, peduncul. sulcatis, cal. hirsuto, fructib. stylo ipsis breviore uncinato-terminatis. S. I. $30 \delta$.

Similis sequenti. 24. Fl. 5,6, 7. In sylvis graminosis rarius. Teg. Kth. Jungfh.

11. $R$. acris: fol. $3-5$-partitis, partitionib. lobat. dentat. aut inciso-multifidis, caule erecto petiolisque adpresso-pilosis, peduncul. teretibus, calyce subvilloso, fructib. stylo brevissimo suberecto terminatis. S. I. 308 .

In pratis, sylvis etc. frequentissimus. 
$12 R$. bulbosus: fol. radicalibus petiolat. trifid. subpinnatifidis, segment. trifid. inciso.dentatis nedio petiolulato, caule erecto ad collum bulboso, cal reflexo. S. I. 309 .

A. $\frac{1}{2}-1 \cdot 24$. Fl 5-g. In solo arenoso et meliore frequentissimus.

* * Corollis luteis, foliis lobatis multifidisve, achaeniis tuberculatis echinatisve.

13. R. philonotis (Retz): fol. trilobat. trisectisve, lob. obtus. incis. dentat medio petiolato, cal. reflexo, caule basi non bulboso, achaen. ad marginem tubercul. minimis adspersis S. I. 309 .

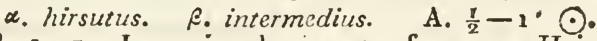
Fl. 5-7. In agris ad vias etc. frequens. Heiligens. Weilss, Charlotıb. etc.

1. $R$. arvensis: fol. glabris, primordialib. apice dentatis, radicalibus tripartitis, caulin. nulifidis lob. linearibus,, caule erecto multifloro, achaen. utrinque longe aculeatis (muricatis.) S. I. 320. A. $\frac{1}{2}-1$ ' . Fol. primaria obovata. F1.5,6. Inter segetes frequens.

\section{3i. CAITHA.}

Cal. o. Cor. inf. 5-petala. Caps. 4-5. 1-locular. polyspermae margine interiore dehiscente es seminiferae, 
1. C. palustris: caule erecto, fol. cordat. suborbiculat. rotunde crenatis, auricul. rotundotis. DC. S. I. 308 .

A. $\frac{r}{2}-1 / 2$. Rad. fibrosa. Fl. 5, 6. Cnr. Iutea. In solo humido et paludoso uudique Erequens.

\section{TroLrus.}

Cal. o. Cor. poly petala. Nect. corona polyphyl!a, foliol. linearibus basi tubulosis. Caps. plurimae 1-locul. polyspermae margine interiore dehiscentes et seminiferae.

1. $T$. europaeus: petal. in globum fere conniventibus, nectar. longitudine staminum. I. A.I.12. A. $1-2,2 \%$. Fol. radicalia longe petiolata 5-partita, laciniis 3 -fid. inciso-dentatis, caulina superiora 5-partita, inferiora 3-partita. Fl. 5, 6. Flos terminalis. Cor. lutea. In prat. humidiusculis passim. Schönh. W. Blankenfelde VV. VVerneuchen. S.

\section{1. XIII. DI D Y N M IA. \\ ORDO I. TOMOGYNIA. \\ 235. A JUGA.}

Cal. 5.fidus subaequalis. Cor. Lubulosa bilabiata, labio super. minimo bidentato (staminib. bre- 
viore), infer. trilobo, lobo medio maximo. Achaen. is.

1. A. pyramidalis: tetragono-pyramidalis, caule villoso, fol. radicalib. breviter petiolat. lato-ovat. leviter repand. villoso-pubescentibus, axillis folior. caulinorum plerumque omnibus foriferis, fol. Horalib. superiorib. integerrimis $v$. nbsolete dentatis floribus longioribus II. A. IX. 19.

A. 3-6" 4. Rad horizontalis, fibrosa. Canlis primum solitarins, serins stoloniferus. Fol. radicalia in orbem disposita, caulina seneim sensimque versus apicem minora, ita ut pyramidis forma exoriatur. Fl. 5. Cor. azurea calyce duplo longior. In sylvis subumbrosis. Gr. Behnitz.

2. A. genevensis: caule villoso, fol, radicalib. breviter petiolat. oblongo-obovat. crenat. pubescentibus, axillis trium parium foliorum caulino. rum Horib. destitutis, fol. floralib. superiorib. tridentat. forib. brevioribus H. A. IX. 18.

Ajuga pyramidalis Kth. 157 .

u. arida B. umbrosa. S. I. 312 . A. $\frac{1}{4}-I^{\prime} 24$. Pad. horizontalis, fbrosa. Folia radicalia pauca, haud raro deficientia. Caulinorum paria tria inferiora plus minusve pedicellata oblongo.ovata. Fl. 5,6. Cor. cyanea, carnea, rarius alba, calyce triplo longior. In solo sicco agro. run sylvarumque frequens. 
3. A reptans: stolonifera, caule villosiusculo, fol radicalib. breviter petiolat. oblongo-obovat. repandis leviter pubescentibus, axillis foliorum caulinorum plerumque omnibus floriferis, fol. floralib. superiorib. plerumque integerrimis Horib. brevioribus H. A. IX, 17.

A. $\frac{1}{2}-1$ 24. Rad. obliqua, fibrosa. Fol. radicalia in orbem disposita. Cor. cyanea, car. nea, rarius alba, calyce triplo longior. In nemoribus sublumidis frondosis hinc inde Jungfh. S. Teg. Schönh. etc.

236. TEUCRIU M.

Cal. 5-fid. subaequalis. Cor. tubulosa labiata, labii superioris loco incisura in qua stamina jacent, labium infer. lobatum, lobo medio maximo. Achaen. 4.

1. T. Scrodonia: fol. cordat. subpubescentibus crenato.dentat. petiolatis, racem. terminalib. axillaribusque secundis, caule erecto berbaceo. L. W. III. 2.i. S. I. 313 .

A. 1-2, 24. Rad. repens. Fl. 6, 7. Cor. ochroleuca. In sylvis frondosis. Thierg. S.

2. T. Scordium: fol. oblong, sessilib. serrat. pubescentibus, florib. axillarib. pedunculatis 2-4, caule erecto pubescenti-villoso. S. I. 313. II. A. VIII. 3.

A. $\frac{\pi}{2}-1 \cdot 2$. Rad. horizontalis articulata. 
Canl. basi stolonifer. Cor. roseo-purpurascens. In locis humidis, pratis, ad margines aquarum etc. frequens. Thierg. Treptow. Teg. etc. Off. 237. NEPETA.

Cal. 5.dentatus, dentib. superiorib. longioribus. Cor. tubulosa incurvata, fauce dilatata margine reflexa, labio super. erecto emarginato, infer. subrotundo crenulato. Achaen. 4 .

1. N. Cataria: incano-tomentosa, fol. petiolat. ovat. cordat. acuminat. grosse serratis, corymb. pedunculat. verticillatis in spicae terminalis formam approximatis. S. I. 314. H. A. IV. 8.

A. $1-2 \frac{1}{2} 24$. Cor, albida, labio inferiore rubro punctato. In ruderatis, ad sepes, vias passim. Teg. S. Weilss.

238. MI NTHA.

Cal. 5-dentatus aequalis. Cor. 4-fida subaequalis, lacinia superiore latiore emarginata. Stam. erecta distantia. Achaen. 4.

1. M. hirsuta: flor. capitat, verticillatisve, fol. petiolat. oratis, cal. undique hirsuto, pedicell. retrorsum hispidis. S. I. 315.

Mentha aquatica L. W. III. 78. Kth. 159.

A. $\frac{T}{2}-1 \frac{T}{2} \cdot 24$. Rad. repens. Fl. 6-8. Cor. extus lirsuta, violacea. In aquis et paludibus frequens. 
2. M. arvensis: flor. verticillatis, fol. petiolat. ovatis, cal. campanulat. undique hirsutis, pilis ho. rizontalibus. S. I. 315 .

Mentha ausiriaca L. W. II. Si. Kth. 139.

A. $\frac{1}{2}-1^{\prime} 24$. Fl. 6-s. Cor. coeruleo-viola. scens. In solo fertili liumido frequentissima.

$$
\text { 239. CIECHOMA. }
$$

Cal. 5-fidus aequalis. Cor. tubulosa fauce villosa, labium super. erectum bifidum, infer. trilobum, lobo medio majore emarginato. Antherarum singulum par in formam crucis connivens. Achaen. \&.

1. S. hederaceum: subglabrum, laciniis calycin. ovat, acutis. S. I. 316 . H A. II. 8 .

Caul, repens. 24. Fol, cordato-reniformia cre. nata. Flor, axillares. Cor, coerulea. In locis humidis et subhumidis undique. Off.

$$
\text { 240. I A MIUIr. }
$$

Cal. 5-dentat, superne dilatatus, dentib. subrequalib. aristatis. Cor. fauce inflata ntıinque dentata bilabiata, labio super. fornicato, infer. bilobo. Achaen. 牛.

1. L. maculatum: fol. ovat. cordat. acuminat. duplicato-serrat. petiolatis, laciniis calycis fructiferi patentibus, corollae labio superiore acuto obsolete dentato. S. I. 317. 
A. $\frac{\mathbf{r}}{2}-1 \frac{\mathbf{r}}{2} \cdot 2$. Fl. 5-8. Fol. saepe maculata. Cor. purpurea. Ad sepes pagos in ruderatis etc. lininc inde.

2. L. album: fol. ovat. cordat acuminat. serrat. petiolatis, petiol. dilatatis, laciniis cal. fructiferi approximatis, corollae labio superiore obtuso in. tegerimo S. I. 317 . II. A. V. 4r.

A. $\frac{1}{2}-2^{\prime} 24$. Rad. repens. Cor. alba. In agris cultis, ruderatis, ad sepes etc. frequens.

3. L. purpureum: fol. ovat. cordat. dentato.crenat. obtus. petiolatis, laciniis calycis fructiferi patentibus, labio corollae superiore obtuso integerrimo. S. I. 318.

A. $\frac{r}{2}-1^{\prime} \odot$. Cor. purpurea minor atque in antecedentibus. In agris, ad sepes etc. frequens. 4. L. amplexicaule: fol. subrotund. obtus. incis. crenatis, inferiorib. petiolatis, foralib. sessilib. amplexicaulibus, laciniis calycis fructiferi conni ventibus, corollae labio superiore obtuso integer. rimo. S. I. 318.

A. $\frac{T}{7}-\frac{T}{2} \odot$. Cor. purpurea. In agris fertilioribus, ad pagos, sepes etc. frequens.

$$
241 . \text { G } \triangle I E O B D O L O N \text {. }
$$

Cal. 5-fidus, laciniis inaequalib. aristatis. Cor. breviter tubulosa fauce patente, labio super. fornicato, infer. 3-fido, laciniis acutis. Achaen. 4. 1. G. luteum. Hudson, S. I. 3:S. Kth. 162. 
Leonurus Galeobdolon. L. WV. III. 115.

A. 3-9" 24. Habitus Lamii. Rad, repens. Caul adscendentes, pilosi. Fol. petiolata, ova. ta, crenato-dentata, pilosa, inferiora cordata. Cor. lutea fance rubro maculata. In sylvis frondosis rarius. Schönh. Briselang.

\subsection{GALEOPSIS.}

Cal. 5-dentatus, dentib. subaequalib. aristatis. Cor. breviter tubulosa, fauce patente, labio super. subcrenato fornicato, infer. 3-lobo supra ad faucem bifornicato-dentatum. Achaen.4. 1. G. Ladanum: internodiis caulin. aegualib. pubescentibus, fol. lanceolat. serrat. supra adpresso-hirtis, subtus molliter villosis, cor. calyce dne plo longioribus, labii inferioris lobo medio latissimo, galea obsolete crenata. S. I. 3Ig.

A. 3-4. $\odot$. Fl. 6, 7. Cor. pubescens, purpurascenti-lilacina et maculis luteis notata. In agris argillaceis passim frequens. Willmersd S. vor d. Hallisch. Th, etc.

2. G. Tetrahit: internodiis caulin. supra incrassatis et hispidis, fol. ovat. lanceolatis utrinque attenuat. crenato-serratis, supra adpresso-hirtis, subtus subpubescentibus, cor. calyce sesquilongioribus, labii inferioris lobo medio subaequali, galea paucicrenata. S. I. 320.

$\boldsymbol{\alpha}$. arvensis, $\beta$. sylyestris. A. $\frac{\pi}{2}-2^{*}$ Caul. non 
pubescens 24. Fl. 6, - Cor. purpurea. In agris ad sepes, pagos etc, $\alpha$, in sylvis subhu. midis 3 . frequens.

3. G. Walterina (Schlechtendal): internodiis caulin. hirto.pubescentib. supra incrassat. et hispidis, fol. ovat. acuminat, crenato-serrat, supra adpressohirtis, subtus molliter pubescentibus, inferiorib. subcordatis, cor. calyce duplo longioribus, labii inferioris Jobo medio subaequali, galea apice dentata. S. I. 320 .

A. $2-3^{\prime} \odot$. Affinis antecedentis formae ๙. Fl. 6, 7. Calyces magis villosi et glandulosi atque in praecedente. Cor. major inten. sius purpurea. In agris, ad pagos etc. Bollensd. Walter. S. 1. 1.

4. G. cannabina (Withering): internodiis caulin. hirt. supra magis et incrassatis, fol. ovat. utrin. que acut. crenato-serrat. utrinque adpresso-hirtis, cor. calyce siiplo longioribus, labii inferioris lo. bo medio latiore, galea apice dentata. S. I. 321.

A. 1-2' $\odot$. Fl. 6, 7. Cor maxima, lutea, labio inferiore inaculis violaceis et lineis fulvis instructo. In agris inter scgetes hinc inde frequens. Friedrf. Trept. etc.

\section{BET O I CA.}

Cal. 5-dentatus, dentib. subaequalib. aristatis. Cor. tubulosa, tubo cylindrico, bilabiata, la. F. B. 
bio super. adscendente planiusculo, infer. 3 -fido. Achaen. 4.

1. B. stricta (Aiton): spica oblonga basi inter. rupta, corollae galea integra, lacinia intermedia labii inferioris erenato-undulata, calycib. pilosis. S. I. 321 .

Betonica officinalis Kth. 163 W. p. n. 615.

A. $1-2 \cdot 2$. Caul. siniplex. Fol. cordatooblonga, obtusa, grosse crenato.dentata, subhirsuta. Cor. subvillosa, purpurea. In sylvis umbrosis hinc inde. Junglh. Teg. Papenb. etc.

\section{STACHYS.}

Cal. 5-fidus, laciniis subaequalib. aristatis. Cor. breviter tubulosa, labio super. fornicato emarginato, inf. trifido, lacinia media latiore emarginata, lateralibus dellexis. Stam. minora deflorata ad latera curvata. Achaen. 4 .

1. S. sylvatica: rad. repente, caule erecto lirto, pilis patulis, fol. petiolat. cordat. ovat. acuminat. hirtis, verticill. 6-foris. S. I. 322. II. A. IV. 11.

A. $1 \frac{\pi}{2}-3^{\prime} 24$. Fl. 6, 7. Cor. sanguineo-purpurea. In sylvis humidis, ad sepes etc. Thierg. Teg. Papenb.

2. S. palustris: rad. repente, caule erecto hirto, pilis reflexis, fol. subsessilib. lineari-lanceolat. supra glabriuscul. subtus pubescentibus, verticil. lis subsexlloris, S. I. 322. H. A. IIJ. 40, 
A. 3-4' 24. Fl. 6, 7. Fol. serrato-crenata. Cor. pallide purpurea. In aquis et paludibus frequens.

3. S. recta: rad. lignescente fibrosa, caule ad. scendente subvilloso, villis patentibus, fol. subpetiolat. elliptico-lanceolat. subhirtis, verticill. multifloris. S. I. 323. H. A. IV. 12.

A. 1-3. 24. Fol. crenata. T1. 6, 7. Cor. Iutescens punctis rubris. In collibus graminosis. Hasenh. IVillmersd. S. Kalkb.

4. S. arvensis: rad. annua, caule erecto hirto, pilis rellexis, fol. ovato.cordat. crenat. subhirtis, inferiorib. petiolatis, calyce corollam subaequante, verticill. sexfloris. S. I. 324 .

A. $\frac{\pi}{4}-1^{\prime} \odot$. Fl. 6, 7, 8. Cor. parva rubicunda, maculis et lineis intensioribus. In agris inter segetes rarius. Zw. Fasanerie u. Charlottb. Ruthe. S. 1. 1.

\section{BALIOTA.}

Cal. hypocrateriform. 5-dentat. 10-striatus, Cor. breviter tubulosa, labio super. concavo cre. vato, infer. 3-fido, lacinia nedia majore eraar. ginata. Achaen. 4 .

1. B. vulgaris (Link): fol, cordato-ovat. serrat. hirsutis, cal. subcylindricis, laciniis acuminat. cuspidatis. Link. Fl. port. I. 115 S. I. 324. Ballota nigra, Kth. 164. B. alba W. p. n. Gra 
A. $1-3^{\prime}$ 24. Fl. 6-S. Cor. purpurea v. al. ba. In ruderatis, ad sepes, muros etc. fro quens.

246. MARRUBIUM.

Cal. hypocrateriform. rigid, 10-dentat. 10-striatus, dentib. altern. minorib. evanescentibusve. Cor. tubulosa bilabiata, labio super. lineari recto bifido, infer. trifido. Achaen. 4 .

1. $M$. vulgare: fol. subrotundo-ovat. dentat. rugoso-venosis, dentib. calycin. setaceis uncinatis. S. I. 325 .

A. 1-2, 4. Fl. 6, 8. Cor. alba. Herba 1ota incana. In pagis, ad sepes, vias etc. fre. quens. OfF.

247. LEONUTUS.

Cal. 5.gon. 5-dentatus. Cor. tubulosa bilabiata, labio super, villoso plano, infer. 3-lobo, lobo medio integro. Achaen. 4 .

1. L. Cardiaca: fol. grosse serratis, inferiorib. subcordat. trilobis, supericrib. basi cuneiformib. trifid. $\nabla$. integris, cor. calyce pungente longioribus, lacinia media labii inferioris acuta. H. A. III. 41. S. I. 325 .

A. $2-4^{\circ} 4$. Fl. 6, 7, 8. Cor, pallide pur. purea. In ruderatis, ad vias, pagos etc. copiosus. 
2 L. Maubiastrum: fol. oblong. dentatis, cor. calyce subpungente vix longioribus, lacinia me. dia labii inferioris subrotunda. S. I. 325.

A. $2-5^{\prime} 24$. Fol. radicalia cordata v. rotunda, obtuse dentata. Fl. 7, s. Cor. parva, ru. bra. In ruderatis et pagis rarior. Rüdersd. W.

\section{2:18. CIINOPODIUM.}

Involucr. multisetum verticillo subjectum. Cal. bilabiatus, labio super. 3-fido, infer. bipartito. Cor. tubulosa superne dilatata bilabiata, labio super. plano emarginato, infer, trifido, lacinia media emarginata. Achaen. 4.

4. C. vulgare: capitul. verticillatis, bracteis setaceis hispidis, fol. superne pilos. remote dentatis, caule subsimplici. L. W. III. 133.

A. 1-2' 24. Fol ovata, acuta. Fl. 6,7. Cor. purpurea. In sylvis et ad sepes hinc inde. Teg. Thierg. Papenb. etc.

\section{ORIGONANUM.}

Bracter calyce major. Cal. varius 5-dentatus aut subbilabitus. Cor. subtubulosa fauce depressa, labio super. emarginato plano, infer. 3-partito, laciniis subaequalibus. Achaen. 4. (Flor. in spicas subtetragonas dispositi).

1. O. vulgare: caule herbaceo, fol. petiolat, ovat. 
acut pubescentibus, spic. subrotund. in corym. bos congestis, bracteis oblongo-ovalib. acut. glabris. II. A. VIII. S.

A. 1-2, 24. Fl. 5, 6. Cor. rosea. In nemoribus graminosis passim, Briselang. S. Papenb. Kalkb. O\&f.

\section{TIIMUS.}

Cal. bilabiatus, fauce villis clausa, labio super. 3-dentato, inf. bifido. Cor. bilabiata, labio super. emarginato, infer. 3-fido, laciniis aequalibus. Achaen. 4.

1. T. Serpyllum: dentib. calycin. inatqualibus, superiorib. ovat, adscendentibus, inferiorib. linearib. porrectis, rerticill. multiflor. subcapitatis, fol. planiusculis obtusis basi attenuat. ciliatis, caulib. repentibus. S. I. 327 .

*. latifolius, 6 . angustifolius. A. $3-6^{\prime \prime} 24$. Fl. 5-8. Cor, purpurea v. albida. Planta variabilis in solo arenoso undique frequens. Of.

x. $T$. Acinos: calycis striati gibbi dentib. inaequalib. adscendentibus, superiorib. lanceol. acuminatis, inferiorib. linearibus, verticill. 6-floris, fol. plan. utrinque acut. subserratis, caulib. ad. scendentibus. S. I. 327.

Acinos vulgaris. Persoon Syn. II. 131.

A. 3-9" $\odot$ et $\sigma^{\top}$. Fol. oblongo.lanceolata, 
acuta. Cor. pallide violacea punctis albis et Gavescentibus. In agris, ad vizs etc. frequens.

2jI. SCUTELIARIA.

Cal. 2 -labiatus, labio utroque truncato, super, obtuse calcarato dein calycem claudente. Cor. breviter tubulosa, fauce trigono-compressa, labio super. fornicato trifido, inf. rotundato emarginato. Achaen. 4.

1. S. galericulata: caulis pube reversa, fol cordato-lanceolat. serrato.crenatis, flor. axillaribus. H. A. III. 36, S, I. 328 .

A. 4"-2' 4. Rad. repens. Fl 6-9. Flor, secundi. Cor. violascenti-coerulea. In locis humidis et in aquis frequens.

$$
\text { 252. PRUNELLA. }
$$

Cal. bilabiatus, labio super. truncato 3-den. tato inferiore 2-fido Cor, breviter tubulosa, bilabiata, labio super. fornicato, infer. trifi. do. Filam. furcata apice inferiore antherifera. Achaen. '́.

1. $P$. vulgaris: canle adscendente, fol. petiolat. oblongo-ovat. basi dentatis, calycis labio supe. riore truncato 3-aristato, cor. calyce duplo majoribus. S. I. 329. II. A. VI 10

A. $\frac{r}{4}-1$ ' 2\%. Fl. 5-9. Flor. spicati. Cor. 
violacea. In locis humidis graminosis fre. quentissima.

2. P. grandiflora: caule adscendente, fol petiolat. oblongo-ovat. basi serratis, calycis labio superiore trifido, cor. calyce triplo longioribus. S. I. 329 .

Habitus partium major atque in antecedente. 24. Fl. 6, 7. In collibus graminosis argillaceis et calcareis. Papenb. W. Kalkb.

253. VERBENA.

Cal. 5-dentatus, dente unico breviore. Cor. infundibuliform. subaequalis curvata, limbo 4-fido, lacinia superiore emarginata. Achaen. 4. immatura membrana suburceolari bifida inclusa, dein libera.

1. V. officinalis: caule erecto, fol. pinnatifidoincisis, laciniis oblong. inciso-serratis, spic. gracilib. paniculatis. H. A. V. 42 .

A. $1-3^{\prime}$ 4. Cor. albido-rosea. In ruderatis, ad sepes etc. frequens.

\section{ORDO II. ATOMOGYNIA.}

254. RHINANTHUS.

Cal. 4-dentat. inflatus. Cor. tubulosa bilabiata, labio superiore compresso fornicato utrinque unidentato, inferiore plano trilobo. Caps. or. 
bicularis compressa 2-locul. 2-valvis, dissepimento medivalvi seminifero.

1. R. major (Ehrhardt): corollae labio superiore emarginato bidentato, dentibus divergentibus, lacinia media labii inferioris brevissina, cal. ovato, capsula ovata mucronata.

Rhinanthus Crista galli a L. IV. III. 1S8. Kth.168. A. $\frac{t}{2}-2^{\circ} \odot$. Fol. lanceolata v. lineari-lanceolata, serratodentata. $F 1.5,6,7$. Cor. Alava macula violacea. In solo humido undique.

2. R. minor (Ehrhardt): corollae labio supe. riore cmarginato bidentatn, dentib. erecto-patentibus, lacinia media labii inferioris brevissima, cal. subrotundo, capsula subroiunda retusa mucro. nata.

Rhinanthus Crista galli. B.L. W.III. 18s. Kth.168. vius.

Antecedenti simillimus et iisdem locis ob. 255. EUPHRASIA.

Cal. subcylindricus 4-fidus. Cor tubulosa bila. biata, labio super. emarginato, infer. 3-lobo. Antherae bilobae, lobis plus minusve inaequa. liter spinosis. Caps. oblonga 2-locul. 2-valvis, dissepimento medivalvi seminifero.

* Antheris barbatis.

1. E. Rostkoviana (Hayne): fol, acute serratis 
calycibusque glanduloso-pubescentibus, stigmate cernuo. H. A. IX. 7 .

Euphrasia officinalis L. IV. III. 193 et Kth. 168. (ex parte).

A. 3-6" ○. Fol. sessilia subrotunda v: lato.ovata. Fl 5, 6, 7. Cor. albae maculis citrinis purpureis et violaceis, rarius coerulescen. tes In locis lnumidis et humidiusculis, in agris, pratis etc. frequens.

2. E. officinalis: ful. argute serratis calycibusque glabriuscul. eglandulosis, stigm. nutante. H A.IX. 8 . a. glabrata, $\beta$. ciliata, $\%$ tenuifolia $H$. $1 . \%$. A. 2-6" $\odot$. Fol. sesilia subrotunda v. lato. ovata v. lanceolata. FI. 6, 7. Cor. fere semper albido-coerulescens maculis flavis et purpureis. In pratis et collibus graminosis, in syl$\nabla$ is etc. frequens.

* * Antheris imberbibus.

3. E. Odontites: fol. lineari-lanceolat. serrat. pu. bescentibus, stigm. erecto, flor. racemos. secundis. S. I. 332 .

A. 2-6" ○. Fl. 6-9. Cal. purpureus. Cor. roseo-purpurascens. In locis humidis et hu. midiusculis, in pratis, agtis etc. frequens.

\section{MELAMPIRUM.}

Cal. 4-fidus. Cor. tubulosa compressa bilabiata, labio super. margine replicato, infer. trifido. Caps, oblique acuminata compressa 2-locul 
subbivalvis, locul. 1-spermis, dissepimento:mo. divalvi seminifero.

1. M. arvense: fol. oblongo-lanceolatis, spic. conicis laxis, bracteis setaceo-pinnatifid. coloratis, cal. scabris. cor. clausis. S. I. 333 .

A. $1-2^{\prime} \odot$. FL. 5, 6. Cor. lutea, apice pur. purascens. In agris fertilioribus inter segetes passim. Spand. W.

2. $M$. nemorosum: fol. nvat. acuminatis, Hor. laxe spicat. bracteat, secundis, bracteis setaceo-dentatis, summ, colorat. sterilibus, cal. lanuginosis, cor. hiantibus. S I. 333 .

A. $\frac{1}{2}-1 \frac{1}{2} \odot$. Fl. 5,6 . Cor. intense lutea. Bracteae superiores steriles violascentes. In sylvis humidis hinc inde frequens. Schönh. Papenb. etc.

3. M. pratense: fol. oblongo-lanceolatis, flor. laxe spicat. bracteat. secundis, bracteis hastatis ez setaceo-pinnatifidis, cal. glabriusculis, cor. clausis. S. I. 33 .

Melampyrum sylvaticum. W. p. n. 638 .

A. $\frac{1}{4}-1^{\prime} \odot$. Fl. 5-9. Cor. pallide Iutea. In solo subhumido et sicciore sylvarum frequen. sissimum.

\section{IA THRAEA.}

Cal. campanulat. 4-fidus. Cor.tubulosa bilabiata, labio super, concavo retuso. Nect. glandula ad 
basin germinis. Caps. 1-locul. 2-valvis, spermophor. singul. medivalvibus.

1. L. Squamaria: scapo simplici, racemo subcernuo secundo, floribus confertis, labio inferiore le. viter trilobo.

A. $\frac{r}{2}-1^{\prime} 24$. Rad. squamosa. Scapi purpurascentes. Fol. O. Fl. 4, 5. Cal. purpureus. Cor. purpurea, labio inferiore albicante. Antherae hirsutae. In sylvis humidis frondosis rara. Tåsd. Ruthe. Schönh. W. S. I. 334.

258. PEDICULARIS.

Cal. 2- . 5-fidus, dente accessorio minimo. Cor. tubuloso-bilabiata ringens, labio super. galeaeformi, infer. trilobo. Caps. obliqua mucronata bilocul. apice bivalvis, dissepimento medivalvi, spermophoro basali subgloboso dissepimento affixo.

1. P. palustris: caule erecto plerumque solitario, fol. pinnatis, foliol. pinnatifido-crenatis, cal, inflat. bifid. cristatis, corollae labio superiore inferius sequante. H. A. VIII. 33.

A. $\frac{\pi}{2}-2^{\circ} \odot$. $F l, 5,6$. Cor. purpurea. In solo humido et paludoso frequens.

2. P. sylvatica: caule simplici aggregato, fol. pinnatis, foliol. dentatis, cal, inflat, inaequaliter 5 -fid. cristatis, corollae labio super, inferius superante. H. A, VIII, 3i. 
A. 3-6" - . Fl. 5. Cor. purpurea. In solo humidiusculo pratorum et sylvarum hino Inde, Charlotib. Jungfh. Friedrf. eic. 259.

259. IINARIA.

Cal. 5-partitus. Cor. personata calcarata, labio super. bifido, palato bipartito, infer. trifido. Caps. bilocul. apice dentibus dehiscens, spermophoro oblongo ntrinque in medio dissepimento affixo.

1. L. vulgaris (Miller): caule erecto, fol. spars. confert. lanceolato-linearibus, racem. subspicatis terminalib. imbricatis. S. J. 337. H. A. VI. 33.

Antirrhinum Linaria W. p. n. 643.

A. $3^{\prime \prime}-2^{\prime}$ 24. Fl. 6-9. Cor. lutea palato vitellino. In agris siccis, collibus, sylvis etc. frequens Gif.

2. L. arvensis: caule erecto, fol. sublinearibus, inferiorib. quaternis, cal. piloso-viscosis, flor, racemosis, calcare recurvo. S I. 333 .

Antirrhinum arvense. L. IV. III. 24 .

A. $\frac{T}{2}-1^{\prime} \odot$. Fl. $T, S_{0}$ Cor. coerulea palato luteo. Herba glanca. In agris argillosis inter segetes hinc inde. Schöneb. Kth. Temph. etc. 3. L. minor: caule ramoso diffuso foliisque lineari-lanceolat. obtus. glanduloso-pilosis, peduncul. axillarib. calyce multoties longioribus. $S$. I. 338 . 
Antirrhinum minus W. p. n. 642.

A. $2-6 "$ C. Fl. 7, s. Cor pallide violacea labio inferiore albo palato flavo. Planta tota viscosa. In muris, tectis frequens, rarius in agris. Opern!. n. Katholische Kirche sonst, auf d. Königl. Schlosse, Churf. Brücke. ctc.

4. L. Fatine: caulib. procumbentib, pilosis, fol. hastat, alternis, peduncul. axillarib. S. I. 339.

Anthirrhinum Elatine L. IV. III. 25 \%.

Fl. 7, 8. - C. Cor. Intea labio superiore vio. laceo. In agiris fertilibus srgillaceis rarius. B. Schönl. u. Panck. Rose Chamisso, S. 1. 1. 26.o. A N TIRIINUN.

Cal. 5-partitus. Cor. personata calcarata basi gibba, palato bijartito, labio infer trifido. Caps. bilocul poris 3 apicalibus dehiscens, spermophoro oblongo utrinque in medio dis. sepimento affixo.

1. A. Orontium: fol. lanceolatis, superiorib. alternis, flor. subsessilibus, cal. corolla lungioriúus. S. I. 339 .

A. 3-6" ○. Fl. 7, s. Cor purpurea palato favo. Rarins in agris fertilibus argillaceis, spand. WV. Weils.

26:. SCROPHUIARIA.

Cal. 5-fidus. Cor. bilabiata resupinata, tubo lere globoso.ventricoso, labio super. 3-fido, iufer. 
2-fido. Stam. erecta compressa 1-locularia rertice fissura dehiscentes. Caps. 2-locul. 2. valv. Spermoph. centrale basalc.

1. S. nodosa: caule 4-gono, angulis acutis, fol. ovat. cordat. duplicato-serrat, in petiolum decursentibu", panicul. terminalibus. H. A. V. 35.

A. $2-3^{\prime} 2$. Rad. tuberculosa. Fl. 6-s. Cor. labium infer. ex fusco lilacinum, super. viridescens. In locis humidis, ad latera aquarum frequens. Thierg. 'Trept. ctc.

2. S. aquatica: caule 4-gono, angul. alatis, fol. ovat. (raro subcordatis) simpliciter serratis in po. tiolum decurrentibus, panicul. terminalibus. If. A. V. 36 .

A. 3-4' 24. Fad. fibrosa. Fl. 6-8 Cor. similis. In locis iisdem antecedente rarior. Ileiligens. Spand. Teg. etc.

3. S. vernalis: caule 4-gono piloso, fol. cordat. pubcscentib. duplicato-serratis, pan. axillarib. dichotomis. S. I. 340 .

A. 1-2' $0^{7}$. Fl. 5. Cor. Intea. In locis humidinsculis, ad sepes prope Schöneb. Thierg. rarius. (Aufuga horti botanici).

\section{DIGITALIs.}

Cal. 5-partitus. Cor. campanulata ventricosa in. aequaliter 4-fida. Caps. bilocul. bivalvis, dissepimentum duplicatum ex marginib. inflexis 
valvularum formatum, Spcrmoph. 2 spongiosa lamina nedium dissepimentum secante conjuncta.

I. D. ambigua: foliol calycin. lanceolatis, cor. galea emarginatis, fol. subtus pubescentibus. In. W. III. 285 .

A. $1-2^{\prime} 24$. Fol. ovata, acuminata, superne glabra. Fl. 6-s. Cor. Alava venis fuscus. In sylvis et nemoribus rarissime. Papenb. W.

$$
\text { 263. I IMIOSELLA. }
$$

Cal. 5-fid, subbilabiat. persistens. Cor. campanulata 5-fida, subaequalis. Caps. bivalv. 1-locul. polysperma, spermophoro centrali ovato fungoso ad basin membrana brevi cum ralvulis cohacrente ceternm libero.

1. L. aquatica: fol. lanceolato-spathulatis, scap, foliis brevioribus. L. W. JII. 34 l.

A. 2-3" $\odot$. Fl. 6, 7. Cor. alba v. rosea. In solo humido, ad margines paludura et lacuum. Temph. IVeilis, etc.

\section{IINNAEA.}

Involucr. 4-phyllum persistens. Cal sup. I-phyllus. 5-partit deciduus. Cor. campanulata 5-fida subacqualis. Caps, baccata 3.locularis, locula. ment. 2. abortivis 1-sperma.

1.L. borealis. (Gronovius), L. W.III. 340. S.I.341. H. A. IV, 13 . 
Caules prostrati 24. Fol. opposita petiolata rotundata serrato-crenata. FI G. Flor. gemini pedunculis filiformib. insidentes. Cor. campanulatae purpurascentes. In sylvis muscosis siccis. Teg. Pichelsb.

Cl. XIT. TETRADYNAMIA.

\section{ORDO I. SILICULOSA.}

265. BERTEROA.

Cal. basi aequalis. Petala limbo bilobo. Stam. minora dentata. Silicula elliptica r. obovata, stylo persistente, valv. planiusculis. Sem. ova. lia subalata. (o二)

1. B. incana (De Candolle): silicul. pubescentibus subventricosis. DC. S. II. 291.

Alyssum incanum

A. $1-2^{\prime} \odot$. Fcl oblonga sessilia. Fl.6-8. Flor. racemosi. Cor. alba. Herba tota pilis stellatis incana. In locis apricis, collibus etc. frequens. Hasenh. Teg. etc.

\section{2fi6. A I Y S SUM.}

Cal. aequalis, Petala limbo integro v. subemar. ginato. Stam. omnia v. nonnulla dentata. Silicula orbicularis v. elliptica, stylo tenui apiculata, valv. planis ve centro convexis. Sem. ovalia. ( 0 )

F. B. 
1. A. calycinum: caulib. herbaceis diffusis, fol, lineari-lanceolat. canescentibrs, cal. persistentibus, silicul. orbiculat. subemarginat. pubescentib. stylo 4-plo longioribus. DC. S. II. 3IE.

A. 2-10" $\odot$. Racemi multiflori. Fl.6, 7. Cor. lutescenti-albida. Planta pilis stellatis incana. In agris arenosis. Kalkb. Templi. Schöneb. etc. 267. EROPIILA.

Cal, aequalis. Petala limbo bipartita. Stam. edentula. Silicula ovalis $\nabla$. oblonga, stigmate sessili, valv. planiusculis. Sem. immarginata. (0 二) x. E. vulgaris (De Candolle): silicul. ellipticis pedicello brevioribus, scapis 5-15-floris. DC. S. II. 356 . S. I. 3 3.

Draba verna. Kth. 37 f.

A. 1-6" $\odot$. Fol. Ianceolata et spatlulata. Fl. 4, 5. Cor, alba. In solo meliore et sabuloso frequens.

268. COCHIEARIA.

Cal. aequalis patens. Petala limbo obtuso. Stam. edentula. Silicula ovato-globosa v. oblonga, valvis ventricosis crassiusculis. Sem. immarginata. (o 二)

1. C. Armoratica: silicul. ellipsoideis, fol. radicalib. oblong. crenatis, caulin oblongo-lanceolat. dentat. incisivisve, rad. carnosa maxima. DC. S. II. 360 . H. A. V. 29 . 
A. 2-3' 2\%. Rad. cylindracea multiceps. Fl. 6. Flor. racemosi. Cor. alba. In ruderatis, agris 'et pratis rarius.

$$
\text { 269. TILASP T. }
$$

Cal. aequalis. Petala aequalia integra. Stam. libera edentula. Silicula depressa apice emarginata, septo oblongo v. ovali, valv. navicula. ribus dorso alatis, locul. 2-polyspermis, Sem. immarginata. (o三).

1. T. arvense: fol. oblong. dentatis, caulib. erectis, silicul: obovato-orbiculat. pedicello breviori. bus. DC. S. II. 375 .

A. $\frac{r}{2}-1^{\prime} \odot$ Fl. 5, 6. Cor. alba. In solo argillaceo inter segetes etc. frequens.

270. CA PSELIA.

Cal. aequalis. Petala integra. Stam. edentula. Silicula depresso-complanata, apice emarginata, triangularis, valv. carinat. dorso non alatis. Sem. plurima. (o =)

1. C. Bursa pastoris (Moench). DC. S. II. 383. S. I. 345 .

Thlaspi Bursa pastoris. Kth. 175.

a. integrifolia, $\beta$. sinuata, $\gamma$. pinnatifida, 8. apetala. A. $2^{\prime \prime}-2^{\prime} \odot$ et $\sigma^{7}$. Fl. 5-2. Cor. alba v. o. Undique conspicua. 
271. TEESDAIIA.

Cal. deciduus, fere 4-partitus. Petala integra inaequalia v. aequalia. Stam. intus squamula aucta. Silicula depressa ovalis emarginata, valv. navicularibus, locul. 2.spermis. Sem. compressa. ( $\mathrm{O}$ 二)

1. $T$. Iberis (De Candolle): petal. inaequalibus, exterioribus majoribus. DC. S. II. 392. S. I. 346. Iberis nudicaulis. Kth. 175.

A. 2-S" $\odot$. Fol spathulata $\nabla$. lanceolata, integra v. sinuata et dentata. Fl. 4,5. Flor. subcorymbosi. Cor, albida. In agris arenosis frequens.

272. C A M EIINA.

Cal. basi aequalis. Petala integra. Stam. edemtula. Silicula obovata globosave, valvul. ventricosis, stylo persistente, locul. 2-polyspermis. Sem. oblonga immarginata. (ㅇ||)

1. C. sativa (Crantz): silicul. cuneato-pyriformib. 4-costat, stylo longiusculo terminatis, fol. subintegerrim. lanceolatis. DC. S. II. 515. S. I. 346. Alyssum sativum. Kth. 175. Myagrum sativum. $L$. A. $1-2^{\prime} \odot$. Fol semiamplexicaulia basi subsagittata. Fl. 6, 7. Flor, racemosi. Cor. luteae. In agris inter segetes frequens.

3. C. dentata (Persoon): silicul. subgloboso-py- 
riformib. 4-costat. stylo longiusculo superatis, fol. repando-dentatis. DC. S. II. 516.

Myogrum dentatum. L. W. III. 108.

Antecedenti similis. $\odot$. Fl. 6, 7. In agris lino consitis frequens.

274. NESIIA.

Cal. aequalis patens. Petala integra aequalia, Stam. edentula. Silicula subglobosa, valv. concavis, septo evanido 1.locul. indehiscens 1-sperma. Sem. subglobosa. (o|l)

1. N. paniculata (Desvaux). DC.S. II.520. S. I. 347. Myagrum panic. L. Aly'sum panic. Kth. 176.

A. $\frac{1}{2}-2 \frac{1}{2}{ }^{\circ} \odot$. Fol. semiamplexicaulia lanceo. Iata v.oblonga, integra v. dentata Fl. 6, 7. Flor. paniculati. Cor. Aava. Planta tota pilis stellulatis tecta. In ruderatis et inter segetes haud rara.

\section{SEN NEBIERA.}

Cal. aequalis patens. Petala integra. Stam. edentula. Silicula didyma aptera bilocularis indehiscens, stigmate sessili, valv. subglobosis, locul. 1 -spermis. Sem. subgloboso-3-quetra, (oll)

1. S. Coronopus (Persoon): fol. pinnati-lobatis, lobis integris dentatis pinnatifidisve, silicul. acur tiuscul. compressis, valv. dorso cristato-rugosis. DC. S. II. 525。 
Cochlearia Coronopus.

A. $\frac{r}{3}-\frac{r}{2}$ ○. Caul. prostrati. Fl: 6, 7: Flor: racemosi. Cor. albae. In ruderatis apricis soli fertilioris rarius. Weilss. S.

275. LEPXDIUM.

Cal. aequalis. Petala integra. Stam. edentula. Silicula ovalis $\nabla$. subcordata, valv. carinatis $\nabla$. rarius ventricosis dehiscentibus, locul. 1-spermis. Sem. compressa v. subtriquetra. (o//)

1. L. campestre (R. Brown): 'silicul. ovat. alat. enıarginat. lepidoto-punctatis, fol. caulin, sagittat. dentatis. DC. S.II. 535. S. I. 348 .

Thlaspi campestre. Kth. 175.

A. 8-10" $\odot$. Fol. oblonga, radicalia sinuatolyrata. F1.5, 6. Flor xacemosi. Cor. albae. Planta tota pilis confertis cinerea. In ruderatis et agris rarius. Stadt, b. d. Hallisch.Th. Teg. Kth. v. d. Schlesisch.Th. S.

2. L. ruderale: silicul. ovat. emarginat. patul. pedicello brevioribus, fo!. glabris, radicalib. pinnatilobatis, rameis linearib. integris, Horib. 2-andris apetalis. DC. S. IJ. 540.

A. $\frac{T}{2}-1^{\prime} \odot$. Fol. radicalia pinmata, pinnis linearibus. Fl. 6-8. Flor, racemosi. Cor. intcrdum 4-petala, petalis llavescentibus. In ruderatis, ad sepes, in plateis etc, frequens. 


\section{TETRADYNAIIA. SILIQUOSA, \\ ORDD IJ. SILIQUOSA. \\ 276. NAsturtum.}

Cal. aequalis patens, Petala integra v. o. Stame edentula. Siliqua teretiuscula abbreviatav. declinata, valv. concav. enerv, non carinatis. Sem. parva immarginata, biserialia. (o $\Rightarrow$ * Petalis albis (Cardaminum).

1. N. officinale (R. Brot $n)$ : fol pinnatifidis, foliol. ovatis subcordatis repandis. DC. S. II. 188.

Sisymbrium Nasturtium. L. H. A. V.32.

A.1-2 24. Caul. procumbens. Fi.6, 7. Flor。 racemosi. Cor. alba anthexis luteis. In fossis, rivulis etc. frequens. Off.

$$
\text { ** Petalis luteis (Brachylobos). }
$$

2. N. syivestre ( $R$ Brown): fol. pinnatis, foliol. lanceolat. serrat incisisve. DC. S.II. 190.

Sicymbrium sylvestre. L. Kth.178.

A. $\frac{r}{2}-1 ' 24$ Rad, repens. Fl. 6-8. Cor, calyce major. Herba glabra. In locis subhumidis, pratis, sylvis etc. haud rarum. Friedrf. Jungth. etc.

3. N. palustre (De Candolle): fol. pinnatilobatis, lobis confluentibus dentat, glabris, rad. fusiformi, petal. calycem aequantibus. DC. II. 191.

Sisymbrium palustre. Kth. 178.

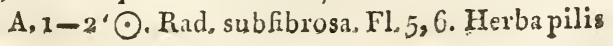


sparsis obsita. In solo humido, ad fossas, paludes etc. frequens.

$4 N$. amphibium (R. Brown): fol. oblongo-lanceo. lat. pinnatifid. serratisve, rad. fibrosa, petal. cal. majoribus, silicul. ellipsoideis. DC. S. II. 196.

Sisymbrium amphibium. Kth. 178.

a. integrifolium. B. variifalium. A. $1-2 \frac{\pi}{2} \cdot 24$. Caul. basi radicantes. Fl.5,6. In locis humidis, paludibus, fossis etc. frequantissimum.

$$
\text { 277. BARBAREA. }
$$

Cal. erectus basi subaequalis. Petala limbo integro. Stam, edentula. Glandulae inter filamenta breviora et pistillum. Siliqua 4-gonoanceps, valvis apice non cornigeris. Sem. in quoque loculo 1-serialia ( 0 二).

1. B. vulgaris ( $R$. Brown): fol. inferiorib. lyratis, lobo terminali subrotundo, superiorib. obovat. dentatis DC. S.II. 206. S. I. 351.

Erysimum Barbarea. Kth. 179.

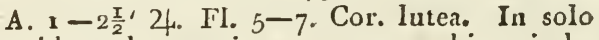
humido ad margines aquarum, hinc inde. Charlottb. Spand.

$$
\text { 278. TURRITIs. }
$$

Cal. laxus, Petala lymbo integro, Stam. eden. tula. Siliqua elongata anceps, valv.planis. Sem。 in quovis loculu biserialia ( 0 )

1. T. glabra: fol, radicalib, dentat, pilosis, caue 
lin. amplexicaulib. integerrimis glabris, siliquis strictissimis pedicello sextuplo longioribus' DC. S. II. 211 .

A. $1 \frac{\pi}{2}-4^{\prime} \sigma^{\top}$. Caul erectus simplex $F 1.5,6$. Cor. albido-flavescens. In solo subhumido sylvarum et collium haud rara. Thierg. Hasenh. Pichelsb. etc.

\section{ARABIs.}

Cal. erectus. Petala limbo integro. Stam. edentula. Siliqua linearis stigmate subsessili coronata, valv. planis, medio 1-nerviis. Sem. 1-se. rialia. (o 三)

1. A. hirsuta (Scopoli): fol. dentatis, pube saepius ramosa scabris, radicalib. obovato-oblongis in petiolum attenuatis, caulin. ovato-Ianceolatis, pedicell. calycis longitudine, siliquis erectis. DC. S. II. 223. S. I. 352 .

Turritis hirsuta. Kth. 1 go.

A. $4^{\prime \prime}-3^{\prime} 4$. Ciul. erectus simplex. Fl. 5, 6. Cor, albida. In solo fertili sicciore in collib. etc. Kalkb. Pichelsb. Papenb. etc.

2. A. Thaliana: fol. pilos, subdentatis, radicalib. petiolat. ovato-oblongis, caule ramoso, siliquis adscendentibus, pedicell. calyce multo longioribus. DC. S. II. 326 .

A. 4"-1' $\odot$. Caul ramosi. Fl. 4, 5. Cor. alba. In solo arenoso undique conspicua. 
3. A. arenosa (Scopoli): fol pube bifurca villosis, radicalib. Lyrato-pinnatifidis, caulin. incisodentatis, caule ramoso pilis simplicib. hispido, pedicell. siliquisque patulis. DC. S. II. 23\%.

Sisymbrtum arenosum. Kth, 178.

A. $1-1 \frac{1}{2}$ ○. Fl. 5, 6. Cor. albae, deflorentes pallide violaceae. (?) In locis arenocis subhumidis umbrosis et apertis, Teg. WV.Schoneb. Kth. Papenb. Charlottb.

2SO. CARDA MINE.

Cal. clausus r. subpatens aeqnalis. Petala limbo integro. Stam. edentula. Siliquae lineares sessiles compressae, valv. plan. enerviis, saepius elastice desilientibus. Sem. ovata 1-serialia immarginata. ( 0 二)

1. C. amara: fol. pinnatifidis, radicalium foliol subrotundis, caulinorum dentato-angulatis, stylo fliformi acutiusculo, caule basi radicante. DC. S. II. 255 . H. A. V. 31 .

A. $1-1 \frac{1}{2} \cdot 24$. Cor. albae antheris violaceis. In solo humido ad fossas et rivulos passin?. Rahnsd. Heide. Jungf h. S.

2. C. pratensis: fol. pinnatifidis, foliol, radicalium subrotundis, caulinorum linearib. lanceolatisve integris, stylo brevissimo vix siliqua tenuio. re, stigm. capitato. DC. S. II. 256. H. A. V. 30. 
A $\frac{T}{2}-1$ 24. Caul. non stolonifer. Fl. 4, 5. Cor. alba antheris havis. In locis humidis umbrosis et apertis undique.

3. C. hirsuta: fol, pinnatifidis, radicalium foliol. subrotund. mucronat. petiolulatis, superiorum oblong. subsessilibus, petal. oblongis, siliquis erectis. DC. S. II. 259.

A. $\frac{7}{2}-1 \cdot \odot$. Fl. 5. Cor. majores atque in antecedente. Omnes partes hirsutae. In solo humido, in pratis etc. rarissima. Rummelsb. Kirchstein. S. I. 355 .

\section{SIS XMBIUM.}

Cal. basi aequal. clausus patensve. Petala integra. Stam. edentula. Siliqua sessilis teretiu. scula, valv. concavis, Sem. ovata v. oblongga -serialia. (o / )

1. S. officinale (Scopoli): fol. runcinatis cauleque pilosis, siliquis rhachi adpressis subulatis. DC. S. II. 459 . S. I. 356 .

Erysimum officinale. H. A. II. 13. Kth. 179. A. $1-2^{\prime} \odot$. Fl. 5-1o. Cor. Havescens. In agris, ad vias etc. undique.

2. S. Sophia: fol, bipinnatifidis, foliol. oblongow linearib. incisis, pedicell. cal. quadruplo longiorin bus, petal, calyce minoribus. DC. S. II. 474, H. A. V. 33 . 
A. $\frac{7}{2}-2^{\prime}$ ○. Fl. $5-9$. Cor. flavescens. In agris, ad vias etc. frequentissimum.

282. ALLIARIA.

Cal. laxus aequalis caducus. Petala limbo obo. vato. Stam. edentula. Glandulae 4. rotundae, 2 cingentes staminum lateraliun basin, 2 inter majora et pistillum. Siliqua teretiuscula lineis prominulis subtetragona. Sem. subcy. lindrica (o $\mid 1$ )

1. A. officinalis (Andrzejowkr): fol. cordatis, siliquis prismaticis pedicello multoties longioribus. DC. S. II. 4 5. S. I. 356 .

Eyssimum Alliaria. H. A. 34. Kth. 179.

A. $\frac{1}{2}-2^{\prime} \sigma^{\top} \cdot \mathrm{v}, 2$. Caulis sparsim pilosus. Fol. infima subreniformia grosse dentata. F1. 5,6 . Cor. alba. In locis humidis umbrosis hinc inde. Briselang. S. Teg. Schönh.

\section{ERYSIMUM.}

Cal. clausus basi subaequalis $\nabla$. subgibbus. Petalc limbo integro. Siliqua valv. carinatis $4 \cdot$ gona sessilis 2-locul. 2-valv. Sem. 1-serialia ovata immarginata. (o/1)

1. E. cheiranthoides: fol. lanceolat. subdenticulat. scabriuscul. viridibus, siliquis erecto-patul. pedicello duplo longioribus, stigm, parvo subses. sili. DC. S. II. 498. 
A. $2^{\prime \prime}-2^{\prime} \odot$. Fl. 5-9. Cor. Hava. In ruderatis, ad sepes, nuros etc. vulgare.

$$
\text { 2S化 BRASSICA. }
$$

Cal. aequalis erectus, raro semipatens. Petala limbo obovato. Stam. integra. Siliqua teretiuscula 2-locul. 2.valvis, stylo parvo brevi obtuso terminata. Sem. uniseriata. $(0)>)$

1. B. olerucea: fol polline glaucis subcarnos. re. pandis lobatisve etiam juniorib. glaberrimis. DC. S. II. 583 .

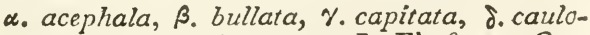

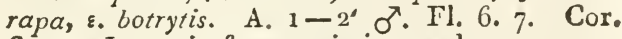
Alava. In agris frequentissime culta.

2. B. Rapa: fol. radicalib. lyrat. polline glauco destitut. setoso-scabris, caulin. mediis incifis, summ. integerrim. laevibus. DC. S II. 590 .

u. depressa $\beta$. oblonga, $\gamma$. oleifera. A. $1-1 \frac{1}{2}{ }^{\top} \sigma^{7}$. F1. 5-9. Cor. pallide llava. In agris, ruderatis etc. vulgatissimum.

3. B. Napus: fol. glabris polline caesio glaucescentibus, radicalib. Iyratis, caulin. pinnatifid. crenatisve, summ. cordato.lanceolat. amplexicaulibus, siliquis divaricato-patentibus. DC. S. II. 592.

$\boldsymbol{\alpha}$. oleifera, $\hat{\beta}$. esculenta. A. $1-\mathbf{2}^{\prime} \sigma^{\nearrow}$. Cor. intense lutea. Herba glabra glaucescenti-viridis. In agris, ruderatis etc. copiosa. 


\section{SINA PIs.}

Cal. basi aequalis patens. Petala limbo obovato integro. Siiiqua teretiuscula bilocularis, bivalvis, valv. nervigeris, stylo parvo acuto rostrata, Sem. 1-serialia subglobosa. ( $>>>)$ I. $S$, arvensis: filiquis glabr, multangul. torulos. rostro tenui subancipiti triplo longioribus, caule foliisq. pilosis. DC. S. II. 615. H. A. II. 14.

A. $1-1 \frac{I^{\prime}}{2}$. Fl. 5-9. Cor. Hava. In agris, ruderatis etc.

2. S. alba: siliquis hispid. patentib. rostro ensiformi subangustioribus, fol. Iyrat. cauleque pilosiusculis. DC S. II. 620. H. A. VIII. 39.

A. $2-3^{\prime} \odot$ : Fl. 6. Cor. flara. Sem. ocliracea. In agris et inter segetes passim. $\mathrm{Vor}$ d. Königsth. S. Ruedersd. Kth. Talsd. Off.

\section{PAPHA N US.}

Cal. erectus, basi subbisaccatus. Petala limbo obovato v. obcordato. Stam. edentula. Siliqua teres transverse multilocularis $\mathrm{v}$. in articulos plurimos secedens, stylo conico acuminata. Sem. 1-serialia globosa. ( o $>>)$

1. $R$. sativus: siliquis teretib. toros, acuminat. vix pedicello longioribus. DC. S, II. 663 .

e. radicula, $\beta$. niger. 'DC. 1. 1. A. 1-1 $1 \frac{1}{2}$ ' et $\sigma$. Fol. lyrato-pinnata pilosa.Fl. 6, $\%$. Cor. 
albidae venis pumpureis $\nabla$. rubro-violacene. In oleraceis et ruderatis passim.

2. R. Raphanistrum: siliq. 1-locularib. articulat. striat. 3-8-sperm. stylo longioribus, fol. simpliciter lyratis DC. S. II. 666. H. A. II. 15.

A: $1-2^{\prime} \odot$. Fl. 5-9. Cor. flava, glandulis 2 nectariferis cylindraceis. In agris ruderatis etc. frequentissimus.

\section{XV. IMONADELPHIA. \\ ORDO I. PENTANDRIA. \\ 2S7. ERODIUN.}

Cal. 5-sepal. inferus. Cor. 5-petat. regularis. Stam. 5. sterilia c. fertilibns alternantia. Nect. glan. dulae ad basin staminum. Achaen. 5 circum styl. posita et aristis spiralib. retrorsum bar. bat, cum eo nexa.

1. E. cicutarium: pendncul multifloris, fol. pinnatis, foliol. pinnatifid. incisis, cor. calyce majo. ribus, caule hirto prostrato v. erecto. L. W. III. 630. S. I. 361.

Geranium Cicutarium L.

A. 2"-1' 4. F1. 5-10. Cor. purpurea. In solo arenoso undique. 


\section{ORDO II. DECANDRIA.}

288. GERAN IUM.

Cal. 5-sepal. infertus, Cor. 5-petala regularis. Nect. glandulae 5 ad basin staminum longiorum. Achaen. 5. circum stylum posita eique aristis nudis simplicibus affixa.

\section{* Pedunculis bifloris.}

1. G. columbinum: peduncul. biflor. folio longioribus, fol. 5-partitis, lob. multifid. linearibus, petal. emarginat. longitudine calycis aristati, achaen. glabr. laevibus, sem. reticulatis. S. I. 362. A. $\frac{r}{4}-1$ ' $\odot$. FI. 6,7. Cor. dilute purpurea. Achaen. olivaceo-plumbea. In solo argillaceo et calcareo hinc inde. Danewilz. Heinersd, S. Rüdersd.

2. G. dissectum: peduncul. bifloris, fol. 5-partitis, lob. 3.fid. linearibus, petal. emarginat. longitudine calycis aristati, achaen. glanduloso-pilosis, sem. reticulatis. S. I, 362 .

Antecedenti simile, a quo differt hirsutie majore, fol. minus divisis, cor. intensioribus, achaen. fuscis. Fl.6.7. In graminosis argillosis ad margines agrorum. Teg. Ruthe. S. 1. 1.

3. G. pusillum: peduncul. 2-floris, flor. 5-andris, petal. emarginatis longitudine calycis mutici, fol. subseptemlob. trifidis, achaen. Laevib. hirsutis, sem. laevibus. S. 1, 362 . 
A. 3-6" ○. Fl. 5, 6, 7. Cor. parva lilacina. In solo sicco meliore, ad ruderata, sepes etc. fiequens.

4. G. molle: peduncul. bifloris, fol. reniformib. radicalib. 9-lobis, caulin 7 -lobis, lob. 3-fidis, petal. bifid. longitudine calycis mutici, aclıaen, gla. bris transversim rugosis, sem. laevibus. S. I. 363. Antecedenti simile, sed fructus et flores majores. $\odot$. Fl. 5-8. Jisdem locis crebro obvium.

5. G. rotundifolium: peduncul, billoris, petal. integris longitudine calycis aristati, fol, radicalib. reniformib. 7-lobis, caulin, subrotund. basi trun. cat. 5-lob. trifidis, achaen. hirsutis, sem, reticula. tis. S. I. 363 .

F1. 5-8. $\odot$. Similibus locis crebro obvium:

6. G. robertianum: peduncul bifloris, fol. ternat. quinatisque trifido-pinuatifidis, petal. integris calyce aristato diplo longioribus, achaen. reticulato-rugosis, sem. laevibus. S. I. 363. II. A. IV. 48. A. 1-3' $\sigma^{\prime}$. Fl. 5-10. Cor. purpurea, rarius alba. In locis sublamidis, in sylvis, ad ruderata etc.

7. G. pratense: peduncul, billoris, fol, subpel. tat. multipartit. rugos. acutis, petal. integr. calyce longioribus, achaen. undique pilosis, scm, punctatis. S. I. 36 f.

F. B. 
A. $\frac{\mathrm{I}}{2}-\mathrm{I}$ 24. Fl. 5, 6, 7. Cor. magna coerulea, rarius alba. Stam. basi valde dilatata In pratis rarius. Teg. W.

8. G. palustre: peduncul. biflor. longissim. declinatis, fol. 5-lobis dentatis, petal integr. calyce aristato duplo longioribus, achaen, undique pilo. sis, sem. laevib. S. I. 364.

A. $\frac{\pi}{2}-1 \frac{\pi}{2} \cdot 24$. Fl. 5, 6, 7. Cor magna purpurea. In pratis, sylvis subhumidis et inter frutices frequens. Jungfh. Teg. Papenb. etc. * Pedunculis unifloris.

9. G. sanguineum: peduncul. 1-fluris, fol. 5-par. tit. Irifid. orbiculatis, petal enarginat. crenulat. cal. aristato duplo longioribus, achaen. laevib, apice pilosis, sem. rugosulis. S. 1. 354 .

A. $\frac{1}{2}-1$ 24. Fl. 5-7. Cor. sanguineo-purpurea. In sylvis frondosis et fruticetis haud rarum, Papenb. Cöpn, ILeide. Kalkb. etc.

\section{ORDO III. POLYANDRIA.}

289. AITHAEA.

Cal. duplex, exterior 2-sepal. 6-12-fidus, inta rior 5-fidus. Cor. 5-petala. Achaen. plura utri. cularia 1-sperma in annulum disposita. 3. A. officinalis: fol, tomentosis, inferiorib. cor- 
datis, superiorib. oblongo-ovat. obsolete trilobis. S. I. 365 . H. A. II. 25 .

A. $2-3^{\prime} 24$. Rad. crassa, lutescens, intus alba. Flor. axillares. Cor. rosea. In pratis ad margines fossarum rarius, Staken. IV. Off.

$$
\text { 29?. MIALVA. }
$$

Cal. duplex exterior j-sepalus, interior 1 -sepal. 5-fidus. Cor.5.petala. Achaen. plura utricularia 1-sperma in annulum disposita.

1. M. rotundifolia: caule subprostrato pubescente scabriusculo, fol. pubescentibus, inferiorib. cordato-orbiculat. obsolete 7-angularibus, superiorib. subquinquelobis, pednncul. pubescentib. fruciiferis declinatis. S. I. 363 . I. A. II. 27.

A. $\frac{1}{2}-2^{\prime} 24$. Fl. 5-10. Cor. carnea. Pili stellati. In plateis, ruderatis, pagis, ad vias etc. frequentissima.

Num M. borealis TFa.Imann S. M. neglecta Wallroth Peichenb. Icon. Plant. rariorum. Cent. I. Dec. If. F. XX. nosirae quoque Florae indigena sit nondum liquet.

3. $M$. sylvestris: canle suberecto piloso scabro, fol. semptemlob. pubescentibus, petiol. pedunculisque erect. pilosis, pet.l. cmarginatis. S. I. 366. H. A. II. 28 .

A $1-4^{\prime}$ 4. Cor. e violaceo purpureoque carnea. Achacn. glabra. In nemoribus, inter frutices elc. copiosa Papenb. Chanlottenb. Talsd. etc. 
3. M. mauritiuna: caule erecto subglabro, fol 5-lob. petiolisque pubescentibus, peduncul, erect. subglabris, petal. retusis. S. I. 366 . H. A. II. 29.

A. $1-4^{\circ} \odot$ Fl. 5-8. Cor. e violaceo pur. pureogue carnea In oleraceis, inter segetes, in ruderatis hinc inde obvia planta australis. Willmersd, etc. S. 1. 1.

4. M. Alcea: caule erecto scabro, fol, scabriusculis, inferiorib. angulatis, superiorib. 5-partitis, calycis foliol. exteriorib. oblongis: S. I. 367. H. A. II. 30 .

A. 1-4' 24. Fl. 5-9. Cor. roseo-purpurea Planta pilis stellatis tecta. In solo argillaceo et calcareo ad sepes, in collibus etc. sparsinı. Döbritz, W. Schulzend, Kalkb.

\section{C1, XVI, DIADELPHIA. \\ ORDO I, HEXANDRIA.}

291. CORYDAIIS.

Cal. 2-sepal. deciduus v. o. Cor. 4-petala papilionaceo-ringens, petalo summo calcarato, infimo ad basin gibbo. Filamenta 2, quodvis antheris 3. Caps, siliquiform. 2-valv. poly sperma, spermoph. 2 suturalibus. 1. C. fabacea (Persoon): rad. bulbiformi solida, 
caule erecto bifido inferne vaginato, fol. duplicato et simpliciter ternatis, foliol. subtripartitis, laciniis integris acutiusculis, racem, terminalibus, bracteis subrotund. integris plerumque flores ao* quantibus. H. A. V. 2.

A. $\frac{1}{2} \cdot 4$. Fl. 5. Cor. roseo-purpurascens. In nemorosis umbrosis, in fruticetis frondo. sis. B. Schulzend. Luch Crome. S. I. 367.

2. C. bulbosa (De Candolle): rad. bulbiformi solida, caule simplici inferne vaginato, fol. biter. natis, foliol. subttipartit. acutiusculis v. integris subrotundis, racemo terminali, bracteis cuneiformibus multifidis pedunculo longioribus. $\mathrm{H}, \mathrm{A}, \mathrm{V} \cdot 3$. Corydalis Halleri. H. l. l. Kth. 187.

F1. 5. Cor. roseo-purpurea, In umbrosis nemorun frondosorum. Schönh. W. Friedrf.W. 292. FUMARIA.

Cal. 2-sepal. deciduus. Cor. papilionaceo-ringens calcarata. Filam 2, quodvis antheris 3. Drupa siliculiformis 1-sperma evalvis.

1. F. officinalis: caule erecto ramoso, fol. tricomposito-pinnatis, foliol. multifid, basi cuneiformibus, pedicell. fructifer, erect. bractea duplo lon. gioribus, drup. subglobosis retusis. H. A. V. 4。

A. $1-2^{\prime} \odot$. Fl. 5, 6. Cor. rosea, apice san. guinea, In solo argillaceo frequens. $\mathrm{OH}_{\text {, }}$ 


\section{ORDO II. OCTANDRIA.}

$$
\text { 293. POIYOAIA. }
$$

Cal. 5-sepal, colorat. persistens, sepal. 2 majorib. alaeformibus. Cor. 1-petala, tubo fisso, limbo 3-fido, lacinia infima supra cristata v. imberbi. Caps. obcordata bilocul. 2-valv. disperma, dissepimento medivalvi.

1. P. yulgaris: caulib. basi procumbentibus, fol. lineari-lanceolatis acutiusculis, flor. racemos, cristatis, alis calicinis obtus. 3-nerv. longitudine corollae. L. NT. III. 873 .

A. $\frac{r}{4}-x^{\prime} 2$. Fl. 5, 6. Cor, violacea, lilacina et alba. In solo subhumido et humido undique frequens.

\section{ORDO III. DECANDRIA.}

$$
\text { 294. SPARTIUII. }
$$

Cal. bilabiatus deorsum productus. Cor. papilionacea, rexillo amplissimo. Stam. connata germini plerumque adhaerentia. Stigm. superne longitudinaliter villosum, Legumen 1.lo. culare compressum.

1. S. scoparium: ram, angulat, inermibus, fol. ternat, solitariisque oblongis, flor, axillarib, soli. 
tariis, leguminibus margine vilksis. L. IV. III. 933. H. A. IX. 10.

A. $2-5^{\prime}$ 万. $\mathrm{Fl} .7$, 8. Cor, vitellina. In so. lo arenoso sylvarum et collium frequens. Thierg. Cüpn. IIeide, Jungfh. etc,

295. GENISTA.

Cal. bilabiatus, labio super. bidentato, infer* tridentato. Cor papilionacea, vexillo oblongo a pistillo et staminib. reflexo. Stam connata. Ĺegumen 1 -loculare compressum. v. inflatum. * Inermes.

1. G. tipctoria: canle erecto, ram. patenti-erectis teretib: striat. glabris, fol. lanceolat. glabris, racem. terminalibus, laciniis calycin, subulatoacnminatis, leguminib glabris. H. A. IX. 11.

A. $1-2^{\prime}$ 7. Fl. 7,8 Cor. flava. In solo arenoso sylvarum et collium frequens. Hasenh. Teg. etc. Off

2. G. pilosa: caule procumbente, ram. tuberculoso-cicatrisatis, fol ovalib. plerumque obtusis, peduncul. axillarib. subsolitar. brevissimis, cor. pilnsis, leguminib pil. brevib. adpress. subscabris. H, F. 132 .

A. $\frac{r}{4}-\frac{\mathrm{I}}{2}$ h. Fl. 5. Cor. flava. In solo arenoso sylvarum et in agris etice osis. Jungfh. Thierg. etc. 


\section{* Spinosae.}

3. G. germanica: ram. annotín. inermibus, reliquis spinis simplicib. ramosisve armatis, fol. lanceolat. acut pilosis, racem. terminalib. nudis, carina inferne ciliata, Ieguminibus pilosis. II F. 133. S. I. 37 \%

A. $1-1 \frac{1}{2}, 77$. Fl. 6, 7. Cor. lutea. In solo fertili sylvarum hinc inde. Hasenh. W. Briselang S. Papenb. Grunw. etc.

$$
\text { 296. on on rs. }
$$

Cal. 5-partitus, laciniis lanceolato-linearibus. Cor. papilionacea, vexillo striato. Stam. sine fissura connata. Legum, 1-locuI, oligospermum turgidum sessile.

1. C. spinosa: ram. spinosis villosis, fol. inferiorib. ternatis, foliol. ovato-lanccolat. serratis, flor. axillarib. subgeminatis. H. F. 135.

A. $1-1 \frac{T^{\prime}}{2}$ h. Fl. 6-10. Cor. dilute purpu. rea. In solo humido et arenoso in pratis, collibus etc. frequens.

297. ROBINIA.

Cal. 4-fidus, Iacinia superiore saepe 2-fida. Cor. papilionacea. Stam 9. coalita, 1 libero, saepius adhaerente. Lcgum. gibbum elongatum.

1. R. Pseudacacia: ram. glabris laevibusque, fol. impari pinnatis, stipal. spinosis, racem. axillari- 
bus, pedunc. 1. Horis, leguminib. laevibus. H. F. 372 .

A. 15-6o' 方 Fl. 6. Cor. alba. In nemori. bus, ad vias etc. frequens.

298. ANTHYLIS.

Cal. 5-dentat. ventricosus. Cor. papilionaces, Stam. basi monadelpha. Legum. calyce inclu. sum I-locul. 1-3-spermum.

1. A. Vulneraria: herbacea, fol. pinnat. inaequalibus, capitulo duplicato. L. W. III. 1013.

A. $\frac{r}{2}-2^{\circ}$ 4. Fl. 6. Cor. luteo-rufescens. In olo sicciore in pratis, collibus, inter frutices hinc inde frequens. Hasenh. Falkenhagen W. Kalkb.

\section{PHASEOLU S.}

Cal. bilabiatus. Cor. papilionacea, carina cum genitalib. in spiram contorta. Legum. oblon. gum 1-locul. polyspermum.

1. P. vulgaris: caule volubili, racemo solitario fol. breviore, peduncul. geminatis, bracteis calyco minorib. patentibus, leguminib. pendulis. L, W. III. 1030 .

Fol. ternata $\odot$. Fl, 6-10. Cor, alba, Culta hinc inde.

2. P. nanus: caule erecto laevi, bracteis calyco 
majoribus, leguminib. pendul. compress. rugosis. L. W. III. 1036 .

A. $1-2^{\prime} \odot$. In agris culta hinc inde. 300. PISUM.

Cal. 5. dentatus, dentib. 2 supcriorib. brevioribus. Cor. papilionacea, vexillo amplo. Styl. 3-angulus supra carinat. pubescens, Legum. oblong. 1-locul. polyspermum.

3. $P$. sativum: petiol. teretibus, stipul. inferrie rotundat. crenatis, peduncul. multifloris. L. W. III. 1070 .

A. $1-3^{\prime} \odot$. Fol. apice cirrhis 3 . instructa pinnata. Fl. 6-8. Flor albitr. purpurei. In solo argillaceo frequenter culta.

2. $P$. arvense: petiol. 4-phyllis, stipul. crenatis, peduncul. 1.floris. L. IV. III. 1071.

Antccedentis forsan varictas, cujus inter segetes frequenter occurrit. Cor. rubro et coeruleo colorata. Sem fusca, non fuscescentiflava, ut in antecedente.

\section{OROBUS.}

Cal. 5-dentatus, dentib. 2 superiorib. brevioribus. Cor. papilionacea, vexillo obcordato. Styl. filiformis. Legum. 1-locul. teretiusculum polyspermum.

1. O. vernus: fol, pinnat, 3-jug. ovat, acumina. 
tis, stipul. semisagittat. integerrimis, caule sim. plici. L. W. 111. 1073.

A. $1-2^{\prime}$ Caulis angulat. glaber 1' $2 \%$. Racemi pancillori $1-2$ ex superiorib. axillis. Fl. 5 . Cor purpurea. In sylvis rarior. Papenb. W. 2. O. tuberosus: fol pinnat. 3-4 jugis lanceo. latis, stipul. semisagittat. integerrimís, caule ala. to. L. W. III. 1074 .

a. angustifolius, $\beta$. latifolius, A. $\frac{r}{4}-1^{\prime} 24$. Rad. tuberosa. Fl. 5, fi. Cor. purpurea. In sylvis acerosis, frondosis, collibus, inter frulices etc. frequens.

3. O. niger: fol. pinnat. tri-sexjugisve ellipticis, stipul. semisagittat. integerrimis, caule ramoso puberulo. S. I. 375 .

A. $\frac{1}{2}-1^{\prime} 24$. Fl. 5, 6. Cor. purpurea. Tota pubescens. In nemoribus et fruticetis sparsim. Grunw. Teg. Papenb.

\section{3ग2. I ATIIYRUS.}

Cal. 5-dentatus, dentib. 2 superiorib. brevioribus. Cor. papilionacea, vexillo amplo. Styl. planus, supra villosus, superne latior, Legum. oblong. 1-locul. polyspermum.

1. L. pratensis: pubescens, caule angulato, fol. pinnat. 1-jugis, foliol. lanceolatis, stipul. lanceol. sagittatis, flor. racemos. ebracteatis, dentib. calycin, subaequalib, acuminatis, S, I. 375. 
A. $\frac{\mathbf{r}}{2}-\mathbf{r}^{\prime} \odot$. Fl. 6-10. Cor. Hava. Tota pubescens In solo humido aperto frequens.

2. L. sylvestris: subglaber, caule alato, fol. pinnat. 1-jugis, foliol. oblongo-lanceolat. subensiformibus, Hor. racemos bracteatis, dentib. calycin. nudis inaequalibus. S. I. $3 ; 6$.

A. 1-2, 2\%. Fl. 6. Cor. purpurea. Hine inde in sylvis et fruticetis. Hasenh. S. Teg. Jungfl.

3. L. palustris: subglaber, caule alato, fol. pinnat. 2-3.jugis, foliol. lanceolatis, Hor. racemosis subbracteatis, dentib. calycin. ciliat, inaequalibus. L. W. 11I. 1090 .

a. angustifolia, $\beta$. latifolia. A. $\frac{\pi}{2}-1 \frac{\pi}{2}^{\prime} 24$. Fl. 6, 7. Cor. magna, purpureo-coerulescens. In solo humido pratorum sylvarumque froquens. Thierg. Cliarlottb. etc.

\section{VICIA.}

Cal. 5.dentatus, dentib. 2-superiorib. brevioribus. Cor. papilionacea. Sty?. linearis sub stig. mate utrinque villosus. Legum. oblong. 1-pluriloculare, dissepiment. membranaceis, poly. spermum.

Racemis pedunculatis.

1. T. cassubica: fol. pinnat. racemo sublongío ribus, foliol. lanceolato-ovat, obtus. mucronat. 
subpuhescentibus, dentib. calycin. inferiorib. li. neari-subulat. tubum aequantibus. S. I. $377 \cdot$

a. villosa, $\beta$. glabrata. A. 1-3' 4 . Fol. 10-15 juga. Fl. 6. Cor. violaceo-purpurea. In sylvis graminosis siccioribus hinc indo Hasenh. S. Teg. Papenb.

2. V. villosa (Roth): fol. pinnat. racemo brevioribus, foliol. elliptic. lanceol. obtusiuscul. mucronat. villosis, dentib. calycin. setaceis, inferiorib. tubo sesquilongioribus. S. I. 378.

A. $1-2^{i} \odot$. Fol. 6-8 juga. Fl, 6, 7. Cor. purpureo-violacea, Tota villosa. Inter segetes Viciae sativae passim. Temph. Cliarlottb. etc.

3. V. Cracca: fol. pinnat. racemo brevioribus, foliol. lanceolat. obtus mucronat. subsericeis, dentib. calycin. inferiorib. lanceolat, acuminat. dimidium tubum aequantibus. S. I. 378 .

A. 1-3.24. Fol. 10-12 juga. Fl. 6, 7, 8. Cor. coeruleo-violacea, In solo humido et argillaceo sicco, ad sepes, in pratis etc.

4. V. tenuifolia (Roth): fol. pinnat, racemo brovioribus, foliol. lineari-lanceol. acut. mucronat. subtus subsericeis, dente calycino infimo subsetaceo, lateralib. ovat. subulato-acuminatis, summ. subrotund. submucronatis S. I. 379 .

Antecedenti similis 24. F1. 6, 7. Cor ex albido et coeruleo varia. Racemi laxiores. In 
collibus inter frutices et ad sepes passim. Ha. senh. W. Bollersd. S. Kalkb.

* Floribus axillarbus, foliis cirrhosis.

5. V. sativa: pubescens, foliol. mucronat. inferiorum foliorum obcordatis, superiorum oblongoobovat. truncatis, stipul. dentat. intus maculatis, leguminib. pubescentib. axillarib. integerrimis. S. I. 379.

A. 1-3' ○. Fol. 3-8-juga. Fl. 6, 7. Cor. e violaceo purpurea. Frequenter in agris culta. 6. V. angustifolia (Roth : subglabra, foliol. oblong. mucronais, foliorum inferiorum obovat. emarginatis, mediorum truncalis, summorum linearib. obtusis, stipul. semisagittat. dentat. integerrimisve, legnminib. pubescentib. axillarib. subgeninis. S I 3io.

๙. syluestris, 3. arvensis. Antecedenti similis C. In agris, rideratis, sylvis etc. frequens.

7. V. lat'y-oides: pubesceus, foliol mucronatis, infimorum folioum obcordatis, summorum lanceolat. obru*is, stipul. orat. semisagitat. integerrimis, leguminib. glabr. axillarib. solitariis $S$. I. 3 so.

Caul. prostrati $\odot$. Fol. 2- S-juga. Fl. 5. Cor. purpurea, parva. In sylvis graninosis siccis. Thierg. Papenb. Schönh. etc.

8. V. sepium: scab:iuscula, foliol. submucrona- 
tis, infimorum foliorum subrotund. ovatisre re. tusis, summorum ovato. lanceolat. obtusis, stipul. ovat. semisagittat. dentatis, leguminib. glabr. axillarib, subquaternis. S. I. 381 .

A. $2-3^{\prime} 24$. Rad. repens. Fol. 4-7-juga. Fl. 6,7. Cor. paliide purpureo-coeru'ea. Cilycis dentes brevissini. In solo meliore hamidiusculo sylvarmm et fruticetorum. Thierg. Jungth. Papenb. Fiedrf. eic.

* * Floribus axillaribus, folïs non cirrhosis.

9. T. Faba: fol. pinnatis, foliol. ovat. integer. rim. glabris, stipul. sagiutat. basi dentatis, leguminib. pubescentib ternis tornlosis. L. WV.111. 1111. A. $1-2^{\prime}$. Fl. 6, 7 . Cor, alba maculis nigris. Iinc iude culta.

\section{ER V U MT.}

Cal. 5.fidus. Cor, papilionacca. Styl. inflexus, apice pubescens, Legum. breve 1.locul. poly- $\frac{\text { g }}{8}$ spermitm.

1. E. hirsutum: peduncul. maltiloris, leguninib. hirsut, dispermis, foliol. lineatib. truncatis. L. WV. III. 1113.

A. 1-2' 9 . Caules debiles angulati. Fo! 6-10-juga cirrhosa. Fl. 6, 7. Flor. racemosi. Cor. albida, parum purpurascens. In agris, ad sepes etc. copiosa. 
2 E. tetraspermum: peduncal. subbilloris, legus minib. glabr. 4,spermis, foliol. oblong. truncatis. L. W. III. 1112.

Antecedenti similis $\odot$. Fl, 6, 7 r Iisdem lo. cis rarius,

\section{GICER.}

Cal. 5-fid. longitudine corollae papilionaceae. Styl. filiformis superne pilosus, Legum. brevo 1.locul. oligospernum.

1. C. Lens (Smith): peduncul. subbifloris, sem. compressis, foliol. integerrimis. L. W. III. 1114. Ervum Lens.

A. $1-2^{\prime} \odot$. Fol pinnata $6-10$-juga. Fl.6,7. Cor. alba parva. Legum. compressa. Culta hinc inde in agris et inter segetes obvenit. 306. CORONIIIA.

Cal. bilabiatus, labio super, bidentato, dentibus approximatis, infer. tridentato, dentib. remo. tis. Cor papilionacea, vexillo alis vix longio. re. Legum. pluriloculare, locul. cylindraceis 1-sperm, articulatim secedentibus.

1. C. varia: herbacea, pinn, plurim. lanceolat, glabris, leguminib. lomentaceis terctib. erectis. L. W. III, 1153 .

A. $1-2^{\prime} 4$. Fol. impari pinnata $8-12 j u g a$. Fl. 5, 6, 7. Flor. capilati. Cor. ex albo roseo- 
que varia. Planta suspecta. In solo argillaceo frequens. Spand Talsd. Reineckend. etc.

$$
\text { 307. ORNITHOYUS. }
$$

Cal. tubulos. 5-dentatus. Cor. papilionacea. Legum. teres arcuatum pluriloculare, locul. 1-sperm. articulatim secedentibus.

1. O. perpusillus: fol pinnatis, flor capitat. bractea snffultis, leguminib. lumenticeis teretiuscul. incurvatis L. W. III. 1155. S. I. 383.

Caules prostrati $9^{\prime \prime}-1^{\prime} \odot$. Fol. impari pinnata 6-12jugi. Fl. 6, 7. Cor. parvae ex lutescenti virescentes. In solo arenoso aprico et in sylvis frequens. Teg. WVeifss, Hasenh. etc.

$$
\text { 30S. HEDXSARUTI. }
$$

Cal. 5.dentatus, dentib. subulatis. Cor. papilionacea, carina obtusissima. Legum. 1. v. pluriloculare, locul. compress. 1.sperm. articula. tim secedentibus.

1. H. Onobrychis: caule erecto, fol. pinnat. cuneat. glabris, alis calycem aequantibus, leguminib. lomentaceis glabr. 1.sperm. aculeato-dentatis. L. W. III. 1215 .

Flor. racemosi. 2. Fl. 6, 7. Cor. roseo-purpurea. In agris argillaceis passim culta et ad vias linc inde.

F. B. 


\section{GALEGA。}

Cal. 5-dentatus, dentib. subulat. subaequalibus. Cor. papilionacea. Legum. 1-locul. polyspermum lineis obliquis notatum.

I. G. oficinalis: fol. pinnatis, foliol. lanceolat. mucronat. glabris, stipul. lanceolat. sagittatis, legunn. erect, strictis. L. WV. III. 1241. H. A, VI. 34. A. 2-5' 24. Fol. 6-3-jnga. FIor. racemosi. Cor. violaceo-lilacina. All sepes rarius. Teg. Kitl.

\section{ASTFAGALUS.}

Cal. 5-dentatus, dentib. subaequalibus. Cor papilionacea. Legum. marginib. valvularum in. tus flexis magis minusve biloculare. Sem. jle. rumque biserialia.

1. A. glycyphyllus: canlescers prostratus, foliol. glabr. ovat. obtus. mucronatis, stipnl. ovato-lanceolatis, racem. pedunculat. folio brevioribus, le. guminib, subtriquetris arcuatis. L. WT. III. 1276. A. 6"-2" 4. Caul prostrati. Fol. 4-6-juga. Fl. 6, 7. Cor. lutescens. In solo argillaceo et arenoso, in collib. sylvis hinc inde. Hasenl. Teg. Papenb.

2. A. hypoglottis: caulescens procumbens, foliol. oblong. obtusis, spic. ovat. pedunculat. folio longioribus, leguminib, erect, ovat, canaliculat. pilosis. L. W. III. 1285 . 
A. 3-4" 24. Fol. 8-10-juga. Fl. 6. Corpurpureo-violacea. Ad latera viarum et in collibus rarius. Kalkb. Ruthe. Cöpnick. Lellmann. S. I. 356 .

3. A. arenarius: caulescens ramos, prostratus, foliol. lineari-lanceolat. sericeis complicatis, racem. subsexflor. folio longioribus, legum. oblong. pubescentibus. L. W. III. 1289.

a. sabulosus, $\beta$. syltaticus. Rad. repens. 24 Fol. impari pinnata 3-j-juga. Fl 6,7 . Flor. racemosi. Cor, pallide violaceo-purpurea. In sterilibus siccis arenosis et sabulosis campo. rum. Hasenh. Wedding. Rüdersd, etc.

$$
\text { 311. SIELIXOTUS. }
$$

Cal. j-dentat, persistens. Cur. papilionacea decidua, carina adpresia. Legum. calyce non tectum 3-loculare oligospermum superne dehiscens decilum (Fol. teruata).

1. M. officinalis (Lamark): leguminib. racemos. nudis disperm. subrugos. ovat. compress. acutis, stipul. subulatis, canle erecto, foliol. subtruncat. serratis, inferiorib. oboratis, superiorib. lanceo. lato-linearibus. W. Enum. 79n.

Trifolium officinale H. A. II. 31 .

A. $2-3$, 2. Fl. 6-8. Flor racemosi. Cor. lutea. In ruderatis intes segetes, circun pagos sparsim. Off. 
2. M. vulgaris (Hayne): legnminib. racemo:. nud. monosperm. rugos. obovat, acutis, stipul. setaceis, caule erecto, foliol. subtruncat. serratis, inferiorib. subrhombeis, superiorib. lanceolatis. IV. Enum. 790.

Trifolium vulgare $H . A . I I .32-$

A. $2-3^{\prime} 4$. Fl. 6-8. Flor racemosi. Cor. alba. In agrisargillaceo-arenosis, ad sepes etc.

312. TRIFOLIUNT.

Cal. 5-dentat. persistens, Cor. papilionacea persistens, carina adpressa. Legum. calyce corollave tectum 1-locul. oliguspermum indehiscens ( $F o l$. ternata).

* Corollis purpureis (Trifolium).

1. T. pratense: spic dens. ovatis, calycis dento infimo tubo corollae 1 -petalite inaequalis breviore, stipul. aristatis, fol. oralib. integersimis, caule adscendente. L. WT. III. I366.

A. $\frac{1}{2}-2^{\prime}$ 24. Fl. 5-20. Flor capitati. In locis humidis et subhumidis undique frequentissimum.

2. T. medium: spic laxis subglobos. solitariis, calycis dente infimo tubum corollae 1-petalae subaequalis aequante, stipul. subulatis, foliol. elliptic. tenuissime serrulat. striato-renosis, caulib. ramos. lexuosis. L. W. III. 1367. 
Affinis antecedenti \& Foliola augustiora. In solo argillaceo, ad vias, in collibus etc. Teg. Kalkb. etc.

3. T. alpestre: spic dens. subglobos. subgeminatis, calycis dente infimo tubum corollae 1-pe. talae subacqualis aequante, stipul. setaceis, foliol. lanceol. tenuissima sermlat. striato-venosis, cau. lib. simplicissim. strictis. L. TV. III. 1368 .

A. $\mathbf{I}-1 \frac{\mathrm{T}}{2}^{\prime} 24 \mathrm{Fl} .5,6,7$. In solo argilloso et subhumido nemorum et collium finc inde frequens. Teg. Papenb. Cöpn. Heide etc.

* Corollis carneis albisve.

+ Calycibus corollam superantibus (Lagopodium).

4. $T$. arvense: spic. villosissim. subcylindraceis, dentib. calycin. setaceis corolla longioribus, foliol. obovato-linearibus. L. W. III. 1373.

A. $2-S^{\prime \prime} 2$. Fl. $6-10$. Planta tota plus minusve villoso.pubescens. In agris et arvis are. nosis frequens.

it Calycibus fructiferis vesiculoso-inflatis (I esicarium).

5. T. fragijerum : capitul. subrotundis, cal. frutiferis inflat. membranaceis pubescentibus, dentib. calycin. setaceis bin. reflexis, foliol. obovat. ob. iusis, caulib. repentibus. L. W. III. I380.

Fl. $6-9^{\prime \prime} 24$. In pratis humidis frequens. 
$\div \div$ Corollis post inflorescentiam deflexis.

a) Capitulis umbellaribus (Trifoliastrum).

6. T. repens: capitul, umbellaribus, leguminib. 4-spermis, dentib. calycin. superiorib. brevioribus, foliol. obovat. emarginat. serrulatis, caule repente. L. W. III. 1359 .

Habitus antecedentis. 24. Fl. 6-10, Cor. alba $v$. rubicunda. In locis graminosis, ad vias, in sylris etc. frequens.

7. T. hybridum: capitul. umbellaribus, leguminib. 4-spermis, dentib. calycin. subaequalibus, foliol. obovat. emarginat. serrulatis, caule adscen. dente. L. W. III. 1359.

Priori similis. 24. Fl. 6-10. Cor, semper rosea. In locis antecedentis rarior.

b) Capitulis spicatis.

8. T. montanum: spic. subimbricat. subtribus, vexill. subulat, emarcescentibus, foliol. ovalib. seriulatis, caule erecto. S. I. 392.

A. $\frac{1}{2}-1$ '24. Fl. 6-8. Cor. alba. Tota planta sericeo-pubescens. In graminosis siccis et humidis praesertim in solo argillaceo. Rum. melsb. Papenb. Kalkb.

$$
\text { * Corollis aureis. }
$$

9. T. agrarium: spic, ovalib, imbricatis, vexill. 
defles persistentibus, dentib. calycin. subnlat. inaequalib. glabris, canle erecto, foliol. lanceolatocuneatis, intermedio sessili. L. IV. III. 1352.

A. $\left.\frac{1}{2}-1 \frac{11}{2} \odot . \Gamma\right] .6,7$. In solo fertili agrorum ex nemorum passim. Weilss. Clarlottb. spand. etc.

10. T. procumbens: spic. ovalib. imbricatis, vexill. deflex. persistentibus, dentib. calycin. subulat. inaequalib. glabris, ram. iprocumbentibus, foliol. oborato.cuncatis, intermedio longius petiolato. L. V. III. 1383 .

T. agrarium $W \cdot$. p. n. 75. . T. campestre. K'th. 200 . A. 3-9" ๑. Fl. 5, 6, 7. Cal. juniores pilosi. In agris fertilib. inter segetes, in pratis etc. frequenter obvius.

11. T. filiform: spic. capitat. hemisphaericis, vexill. deflex. persistentibus, dentib. calycin. subulat. inaequalib. glabris, caulib. procumbentibus, fol. obovato-cuneatis, intermedio longius petiolato.

Praecedente gracilius. $\odot$. Fl. 5-7. Vexillum angustius, carinatum non sulcatum. Si. milibus locis obvinm,

\section{LOTUS.}

Cal. tubulos. 5-fidus, laciniis aequalibus. Cor. papilionacea, carina superne clausa. Legum。 
multiloculare cylindricum rectum polyspermum, dissepimentis spongioso-membranaceis. 1. L. corniculatus: capitul. depressis, caulió. decumbentibus, legum. cylindraceis patentibus. L. W. III. 1395.

L. uliginosus et L. arvensis. Kth. 201.

u. collinus, $\beta$. pratensis, $\%$ palustris. A. $\frac{\mathbf{r}}{2}-3^{\prime}$ 24. Fol. ternata. Fl. 5-1n. Cor. vitellina. In agris, pratis, collibus frequentissimus.

314. MEDICACO.

Cal. subcampanulat. 5-fidus, laciniis aequalibus. Cor. papilionacea, carina superne clausa de. mum fissa. Legum. 1.multilocul. 1.v. polyspermum curvatum falcat. $v$. cochleatum.

* Leguminibus $\mathbf{1}$-locularibus $\mathbf{1}$-spermis (Lupulina)

1. M. Iupulina: spic. ovalibus, leguminib, reniformib. I-spermis, stipul. integerrimis, fol. obo. ratis. L. IV. III. I 406.

A. $\frac{1}{2}-2^{\prime}$ o' Fol. ternata. Fl. 5, 6, 7. Cor. lutea. Planta tota pubescens. In solo melioro in pratis, agris etc copiosa.

* Leguminiblis plurilocularibus poly. spermis.

+ Leguminibus subcontorto-falcatis (Medicago).

2. M. sativa: peduncul, racemosis, le uminib. 
laerib. cochleato-contort. glabris, stipul. subden. tatis, foliol oblong. dentatis. L. IV. III. I404.

A 2-3 24. Fol. teruata. Гl. 6, 7, x. Flor. racemo i Cor, violacen. In ogris et pratis pas. sim. Friedrf. W'eifis. Teg. etc.

3. M. falcata: peduncul, racemosis, leguminib. contorto-falcat. pubescentibus, stipul. subdentatis, foliol. oblong. emarginat. apice dentatis. L. WV. III. 1405.

Ilabitus antecedentis 4 . F!。 5, 6, - . Racemi subcapitati. Cor. flavae. In solo argillaceo et subbumido, in agris, pratis frequens.

++ Leguminbus coulleatis (Medica).

4. M. minima (Willdenow : peduncul. multifloris, leguminib. cochleat. pilosis, aculeis rect. subulat, uncinatis, stipul. integerrimis, foliol. obo. vat. dentatis. L. W. III. 1418.

A $\frac{1}{2}-1^{\prime}$ ○. Caul prostrati. IJ. 5, 6. Racemi axillares. Cor. parvae flave. In collibus agrisque argillosis. Hasenh. WV. Kalkb.

\section{C1, XVII, POLYADELPHIA. \\ ORDO I. POLYANDRIA.}

315. HYPERICUM.

Cal. inf 5-partitus. Cor. 5-petala. Caps. 3-5-10. cularis, apice 3-5.Valvis, dissepiment dupli. 
cat. ex inflexis marginib. valvarum formatis. Spermoph. centrali columnari semina et dis. sepimenta sunt affixa.

* Trigyna, calycibus integerrimis.

1. H. quadrangulare: caule herbaceo quadrangulari, fol. obtus, pellucido-punctulatis, for. trigynis, laciniis calycinis lanceolat, acutis. L. W. III. 1459. H. A. VIII. 43.

A. $1 \frac{t}{2}-2 \frac{\pi}{2}$ 24. Rad. repens. Fol. obovata. Fl. 6, 7. Flor. corymbosi. Cor. cirrina. In solu hnmido graminoso frequens. Thierg. Teg. Heiligens, etc.

2. H. perforatum: caule herbaceo subancipiti, fol. obtus. pellucido-punctatis, for trigynis, laciniis calycin. lanceolat. acutis. L. W. III. 1460. H. A. VIII. 42 .

Autecedenti simile.24. Cor. intensius colorata. In solo argillaceo et arenoso undique. Off.

3. H. dubium (Leers): caule herbaceo obsolete 4-angulo, fol. obtus. epunctatis, flor. trigynis, laciniis calycin elliptic. obtusis. L. W. III. 1,460.

Medium tenet inter $H$. perforatum et quadrangulare 2\%. Caul. sub foliis 4-angulus supra anceps. In solo argillaceo in nemoribus et ad sepes. Teg. Papenb. etc.

4. H. humifusum: caulib. (procumbentib.) herbaceis ancipitibus, fol. obtus, pellucido-punctula- 
tis, flot. trigynis axillarib. terminalibusve, laciniis calycin. ellipticis. L. WV. III. 1/461.

A. 3-6" 24. Fol. parra clliptica v. sublanceolata. Fl. 6-10. Flor. pedunculati. In agris sterilibus arenosis et sublimidis hinc inde. IVeifss. Papenb. Teg. etc.

* Trigyna, bracteis calycibusque serratoglandulosis.

5. H. montanum: caule ereti erecto, fol. ovatooblong. amplexicanlib. impurictat, margine nigromaculatis, for. trigyn. corymboso-paniculat. confert, teminalibus, cal. serrato-glandulosis. L, W. III, 1463 .

A. $2-3$ ' 24. Fl. 6, 7. Cor. magna citrina, In sylvis frondosis, Kalkb. Kth. Teg. Jung fh. Papenb. S.

\section{C1. XVIII. SYNGENESIA. \\ ORDO I. AEQUALIS.}

316. TRAGOPOGON.

Involucr. longe pyramidatum simplex sub-io-fidum aequale. Receptac. nudum excavato-puntatum. Papp. plumosus stipitatus. Achaen. praelonga. 
1. T. pratensis: involucro flores extimos ae. quante, pedunculo tereti, fol. integris carinat. acuminat. basi dilatatis, axillis nudis. S. 1. 399.

a. elatior, 3 . humilis. A. $\frac{1}{2}-3^{\prime} \sigma^{\nearrow}$ et 4 Fol. lineari-lanceolata. FI. 6, 7. Cor. Havae. In solo argillaceo frequens, ad vias, in collibus etc.

2. T. major: involucro flores extimos superante, pedunculo snperne incrassato, fol. integr. carinat. acuminat. basi dilatatis, axill. lanuginosis. S. J. 400. Antecedenti similis ơ. Fl. 6, 7. Cor. pallidiores. Ful. anginstiora. In solo argillaceo et calcareo tarior:" Spand. IV. Kalkb.

317. S CORzONERA.

Involucr. oblong. polyphyll. imbricatum foveolat. acuminat. niargine scarios. inaequalibus. Re. ceptac. nudun papillosum. Papp. plumosus sessilis.

1. S. humilis: caule subnudo subsimplici, fol. oblongo-lanceolat. nervos. planis, involucri foliol. lanatis. L. W. III. 1.nng.

A. $\frac{8}{2}-2^{\prime} 4$. Fl. 5. Cor. flavae. In sylv. subarenosis graminosis frequens. Jungfh. Grunw. etc.

2. S. rosea (Waldst. Kit.): caule subsimplici, fol. lanceolato-linearib planis, caulin. carinato. 
linearibus, involucri foliol. villosiusculis. L. W. III. 150\%.

A. 1-2' 24. Cor, roseae. In collibus et siccis sylvarum atque collium, Briselang. Teg. Kil. Bamb. S. Jungfl.

318 . Soxcines.

Involucr. polyphyll, imbricat. basi ventricostum. Receptac. nudum excavato.punctatum. Papp. sessilis pilosus.

$$
\text { * Perennes. }
$$

1. S. arvensis: peduncul. calycibusque hispid. subumbellatis, fol. runcinat, basi cordatis. L. IY. III. 1512.

Sonchus palustris. W. p. $n$ 764. Kth. 206. A. 2-4 4 Rad. repens. FI.6-S. Involucr. glanduloso-hispidum. Cor. Ritvae. In agris et pratis frequens.

$$
\text { * Annui. }
$$

2. S. oleraceus: fol. oblongo-lanceolat. integris et runcinatc-pinnatifid. dentat. amplexicaulibus, peduncul. umbellat. involucrisque glabris, achaen. compress. tuberculosis. S. J. 402. H. A. I. 4S.

Sonchus oleraceus a et f laevis. L. W. III. 1514. A. $2-y^{\circ} \odot$. Fl. $6-10$. Cor. pallide flavae. In agris soli melioris, in incultis etc. frequens. 3. S. asper (Willdenow): fol, oblongo.lanceolat. 
integris et runcinato-pinnatifid, dentat. amplexicaulibus, peduncul. umbellat. involucrisque glabris, achaen. compress. marginat. laevibus. S. I. 402.

Soncluus oleraceus $\gamma$ et $\delta$ asper. L. W. III. 1515. Simillimus antecedenti. Iisdem locis.

\section{$3^{19}$ LACTUCA.}

Involucr. polyphyllum imbricat. oblongum, foliol. inaequalib. margine scariosis. Receptac. nudum excavato-punctatum. Papp. stipitatus pilosus.

1. L. sativa: fol. rotundatis, caulin. cordatis, caule corymboso. L. IV. III. 1583.

A. ${ }^{-2} 2^{\prime} \odot$. Fl. 6, 7, s. Cor. Navae. In agris ct oleraceis frequenter culta.

2. S. Scariola: fol aversis runcinato-pinnatifid. denticulat. basi sagittat. carina aculeatis. H. A.I. 46 . A. $2-3^{\prime}$ ○. Fl. 7, 8. Cor. citrinae. In solo argillaceo, circum pagos, in ruderatis etc. passim. Thierg. Temph Kalkb. Weifss, Off.

Variat fol. integris, cujus modi specimina prope Weifss decerpsimus, eoque proxime accedit ad $L$. virosam cujus varietas ab Hallero habita est.

320. CHONDRIIIA.

Involucr. duplex, exierius ex foliol. pauc. bre. vib. compositum interius cylindricum, sub 
10-phyllum. Receptac. nudum excavato-punctatum. Papp. stipitatus pilosus.

1. C. juncea: fol. radicalib. runcinatis, caulin. linearib. integris. L. W. III. 1531.

A. 1- 24. Fl. 6-8. Cor. flavae. In solo arenoso, ad vias, in agris etc. frequens.

321. PRENANIIES.

Involucr. duplex, exterius foliola pauca brevis. sima, interius sub-5-phyllum. Receptac. nudum papillosum. Papp. subsessilis pilosus.

I. P. muralis: fol. lyrato-pinnatifid. dentatis, lobo terminali 5-angulari, for. paniculatis. L. W. III. I543.

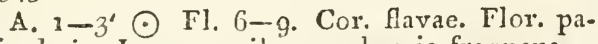
niculati. In nemoribus umbrosis frequens. 322. IEONTODON.

Involucr. duplex, exterius foliola plura breviora inaequalia, interius erecta aequalia. Receptac. nudum excavato-punctatum. Papp. stipitatus pilosus.

1. L. Taraxacum: fol. runcinat. laciniis acutis. L. W. III. 1544. H. A. IV. 4.

$\alpha$. oleraceum, $\beta$. arenarium, $\%$ paludosum, Fl. 5, 6. 24. Cor. vitellinae, Planta notissima undique ovia. Off. 


\section{A PARIA.}

Involucr. poly phyll, imbricatum. Receptac. scrobiculatum villosiusculum. Papp. sessilis plumosus, radiis inaequalibus.

3. A. hispida (Scopoli): scapo nudo 1-floro calyceque hirto, fol. runcinato-dentat. hirtis, pil. furcatis. L. IV. III. 1552,

Leontodon hispidum.

A. $\frac{\pi}{a}-1$ 24. Fl. 6, 7. Cor. aureae. In solo humido, in pratis, ad paludes etc. frequens.

2. A. autumnalis (Scopoli): scapo ramoso declinato, pedicell. squamosis, fol. lanceolat. dentatopinnatifid. glabriusculis, involucro pubescente. L. W. III. 1550 .

Leontodon autumnale.

a.sylvestris, B. campestris. A. 1-2' 24. Fl. 7, s. Cor. aurea. In solo sublumido, in pratis, pascuis etc. obvia.

\section{THR I N C I A.}

Involucr. simplex octangulare s-phyllum, Receptac. favosum. Papp. florum radii: menbrana multifida, disci: sessilis plumosus.

1. Thirta (Roth): scapo simplicissimo, involucro glabriusculo basi squamulis aucto, fol. lanceolat. sinuato-dentat. hirtis, pil. furcat, simplicibusque. S. I. 407 . 
Leontodon hlrtum. W. p. n. 725.

A. $\frac{\pi}{2}-1^{\prime} 4$. Fl. 6-s. Cor. aurea. Habitus A. hispidae. In pratis passim. Hasenh. W. 325. PICRIS.

Involucr. duplex, exterius brevins, interius erectum aequale polyphyllim. Receptac. excava. to-punctatum. Papp. sessilis plumosus.

1. P. hieracioides: caule erecto scabro, fol. am. plexicaulib. lanceol. dentatis, peduncul. corymbosis, involucro externo laxo. L. IV. III. 1556.

A. 2-2 $\frac{I^{\prime}}{2} \sigma^{\top}$. FI, 6, 7. Cor. llavae. In solo pinguiore argillaceo, ad fossas, in agris etc. Panck. Ruthe. S. 1. 407.

326. HIERACIUM.

Involucr. imbricat, polyplyyllum, foliol. inaoqualibus. Receptac. scrobiculatum setulis sca. briusculum. Papp. sessil. pilosus.

* Caule simplicissimo.

3. H. Pilosella: fol. integerrim. obovat. lanceolatisve subtus tomentosis, stolonib. repentibus, caule simplicissimo. H. A. HII. 42. S. I. 408.

A. 3-6" 4. Fl. 5-10. Cor. Havae. In solo arenoso undique frequells.

* Caule apice ramoso.

+ Foliis radicalibus basi attenuatis non petiolatis.

F. B. 
2. H. dubium (Willdenow): caule subrudo sub. glabro, stolonib, repentibus, fol. oblongo-spathulat. lanceolatisve obtusiuscul. integerrim. glabr. ciliatis, capitul. subquatern. terminalib. pedunculatis, peduncul. involucrisque pubescentibus et glanduloso-hirtis. S. I. $40 \mathrm{O}$.

Habitus praecedentis 4 . Fl. 6, 7. Cor. flávae. In locis sublumidis, ad paludes etc. frequens.

3. H. praealtum (Villars): caule oligophyllo subglabro, stolonib. o, fol. oblongo-lancenlat. acut. glabris margine ciliatis, capitul. paniculato-corymbosis, pedicell. involucrisque pubescentib. et glanduloso-hirtis. S. I. 409.

Hieracium florentinum. L. WV. III.1565. Kth. 210. A. $1 \frac{\mathrm{r}}{2}-2^{\prime}$ 24. FI. 5, 6. Cor. Havae. In solo calcareo aprico Kalkb.

4. H. echioides (Lumnitzer): caule oligophyllo hispido, stolonib. o, fol. oblongo-lanceolat. acut. integerrim. utrinque hispidis, capitul paniculatocorymbosis, pedicell. involucrisque pubescentib. et hispido-eglandulosis. S. I. 409.

๙. nemorosum, $\beta$. calcareum. Habitus antecedentis. In locis nemorosis, argillaceis et in solo calcareo. Kalkb. S.

5. H. cymosum: caule oligophyllo hispido substolonifero, fol. lat, oblongo-lanceol, subdenticu- 
lat. subpilosis, corymbo subsimplici terminali, pedicell. involucrisq. pubescentib. hispid. glandulosisque. S. I. 410.

A. $\frac{1}{2}-2^{\prime}$ Fl. 6, 7, 8. Cor. Havae. In sylvis frondosis Briselang. S.

+ Foliis radicalibus petiolatis.

6. H. paludosum: caule folioso glabro, fol. runcinato-dentat. obovato-oblong. acut. glabris, caulin sessilib. semiamplexicaulibus, capitul panicu. latis, pedicell. pubescentibus, involucr. subhispidis. S. I. 410.

A. 2-3، 24. Fl. 5, 6. Cor. Havae. In solo humido, in pratis, inter frutices frequens. Teg. Friedrf. Ta[sd, etc.

7. H. murorum: caule oligophyllo pubescente et subhirsuto, fol. ovat. basi subtruncato-cordatis et profınde dentatis, apice denticulat. subhirsutis, caulino petiolato, capitul. paniculatis, pedicell. et involucr. pubescentibus et hirto-glandulosis. S. I. $41 \mathrm{I}$.

A. 1-2' 2. Fl. 6-9. Cor. flavae. In sylvis siccis et humidis vulgatissimum. Grunw. Jungfh. etc.

8. H. sylvaticum (Gouan): caule oligophyllo pubescente et subhirsuto, fol. ovalib. utrinque attenuat, aequaliter dentat subhirsutis, caulin. petio- 
latis, capitul. paniculatis, pedicell. et inrolucris pubescentib. hispidis et glandulosis. S. I. 411.

A. $\frac{\mathrm{T}}{2}-2^{\prime} 24$. Fl. 6-9. Cor flavae. In sylvis siecis et sirbhumidis frequens. Grunw, Cöpnick. Heide etc.

\section{$+\dagger+$ Foliis radicalibus emarcidis.}

9. H. sabaudum; caule folioso subhirsuto, fol. caulinis ovalibus lanceolatisve utrinque dentatis subhirsutis, capitul. paniculato-corymbosis, pedicellis subsquamosis pubescentibus, involucris laxe adpressis pubescentibus subglandulosis. S. I. 412 .

๙. monocephalum, $\beta$. vulgatum, $\gamma$. latifolium. A. $\frac{x}{2}-4^{\prime} 4$. Fl. 6-8. Cor. flavae. In solo subhumido sylvarum et inter frutices. Jungfb. Weifs, etc.

10. H. umbellatum: canle folioso subglabro, fol. caulin. linearib. utrinque dentat. subglabris, capiul. paniculato-umbellatis, pedicell. squamos. puberulis, involucr, squarros, subglabr, eglandulosis. S. I. 412 .

A. $\frac{r}{2}-2^{\prime}$ 24. Fl. 6-8. Cor. flavae. In solo subhumido et sicciore, in pratis, sylvis, Teg. Friedrf. etc.

327. CREPIS.

Involucr. duplex, exterius breve patulum deciduum, interius basi ventricosum polyphyl. 
lum. Receptac. alveolatum $\nabla$. scrobiculatum pilosiusculum. Papp. stipitatus v. sessilis pilosus.

1. C. virens: peduncul, filiformibus, involucr. pubescentib. subglandulosis, achaen. pappo duplo minorib. utrinque subattenuat. curvatis. S. I, 413

Crepis pinnalifida L. WV. III. 1604. Kth. 211. a. humifusa, B. erecta. A. $\frac{\mathrm{t}}{2}-1 \frac{\mathrm{t}}{2}{ }^{\prime} \odot$ et $\sigma^{7}$. F1 6-9: Cor. Havae. In locis subhumidis graminosis $f$, in agris et collibus siccioribus $\alpha$ frequens.

2. C. tectorum: peduncul, apice incrassat, invo. lucr. pubescentibus et hirto-glandulosis, achaen. pappum subaequantib. apice valde attenuatis, $S$. I. 414 .

a. se.jetalis $\beta$. campestris. Antecedente, cui simillima, in ounibus partib. validior. In agris, muris, etc. copiosissime.

3. C. biennis: peduncul. crass. apice incrassatis, involucr. interiorib. subtomentos. et hispidis, exteriorib. subglabris, achaen. pappum aequantib. apice valde attennatis. S. I. 4140

A. $\frac{1}{2}-2 \frac{1}{2} \sigma^{\top}$. Fl. 6-9. Cor. Havae. In solo fertiliore rarius. Sulünhaus. Panck. Ruthe。 Weifss.

328. HYPOCHOERIS.

Inpolucr. imbricat, polyphyllum. Receptae. pa- 
leaceum. Achaen. difformia. Papp. plumosus, radii sessilis copiosior, disci stipitat. parcior. 1. H. glabro: glabriuscula, caulib. ramos. subfoliosis, fol. dentato-sinuatis, involucr. oblong. imbricatis, L. IV, III. I621.

A. $\frac{1}{2}-1^{\prime}$ 24. Fl. 6, 7. Cor. flavae. In agris arcnosis hinc inde, Hallisch. Thor. Teg. etc.

\section{A CHYROPHORUS.}

Involucr. oblong. imbricat. polypliyllum. Receptac. paleaceum. Achaen. uniformia. Papp. stipitatus plumosus uniformis.

1. A. maculatus (Scopoli): hirtus, caule simplici subaphyllo, fol. oblong. $\nabla$. obovato-oblong. integr. dentatis, peduncul. squamosis. S. I. 415.

Hypochoeris maculata. Kth. 212. H. A. VI. 43. A. $\frac{\mathrm{T}}{2}-1^{\prime}$ Fol. castaneo maculata. Fl. 5, 6. Cor. Alavae. In sylvis graminosis umbrosis. Papenb. W. Cöpnick. Walter. Jungfh. Chamisso.

2. A. radicatus (Scopoli): caule ramoso subaphyllo glabro, fol, oblong. runcinat. obtus. scabris, peduncul. squamosis. S. I. 415.

Hypochoeris radicata. Kth. 212 .

A. $\frac{\mathrm{T}}{2}-1 \frac{T}{2}$ Fl. 6-9. Cor. Havae. In agris, ad vias, in nemoribus etc. haud rara. 


\section{LATSANA.}

Involucr. duplex, exterius foliola panca brevissima, interius 8-phyll. erect. comivens. Receptac. nudum, papill depressis. Papp. O. 1. L. communis: caule paniculato, fol. ovat. petiolat. angulato-dentatis, peduncul. filiformibus, involucr. fructifer. anguiatis. S. I. 416.

A. 1-4' $\odot$. Fl. 6-10. Cor. pallide fava. Ad sepes, in sylvis sublumidis etc. frequentissima.

\section{AR NOSERIS.}

Involucr. simplex polyphyll. p. maturitatem to. rulosum et globose connivens. Receptac, nudum excavato-punctatum. Papp. margo coriaceus erect, integerrimus.

1. A. pusilla (Gaertner): caule nudo simplici, peduncul. fistulosis clavato-incrassatis, fol. obovatooblong. denticulatis. S. I. 416.

Hyoseris minima L. Lapsana pusilla. L. W, III. 1623. Kth. 213.

A. $3^{\prime \prime}-\mathbf{1}^{\prime} \odot$. Fl. $6-9$. Cor. Havescens. In agris arenosis. Cluarlotıb. Spand. Teg. etc.

332. CICHORIUII.

Inpolucr. duplex, exterius breve adpressum, interius longissimum per maturitatem rellexum. Receptac. nudum v. setis obsitum. Papp. squa- 
mae membranaceae $5 \mathrm{v}$. plures achaenio mul. toties breviores.

1. C. Intybus: capitul. axillarib. gemin. subsessilibus, fol. runcinatis. L. W. III. 1628. H. A. II. 24 . A. 1-3' 24. Fl. 6-10. Cor. cyaneae, coeru. leae, albae v. purpureae. In solo sicciore argil. laceo et subliumido frequens etc. Off.

33.3. A R CTIU Mi.

Involucr, globosum poly phyll. imbricatum, squamis apice hamis reflexis. Receptac. paleaceum. Papp. brevis.

1. A. majus (Schkuhr): fol. cordat, utrinque gla. bris subtus canis, capitul. paniculatis, involucri nudi glabri foliol. margine paleaceis. S. I, 117

Arctium Lappa. H. A. II. 35.

A. 3-6. 24. Fl. 6-9. Cor. purpurascens. In sylvis et locis fertilioribus rara. Briselang. S. Off.

2. A. minus (Schkuhr): fol. oblongo-ovat. subtas pubescenti-subtomentosis, capitul. racemosis, involucri subglabri foliol, margine setis acutis obsitis. S. I. 418 .

Arctium Lappa. Kth. 214. (ex parte.)

A. 1-2' 24. Antecedenti simile. Cor, nune albida, nunc purpurea. In ruderatis, ad vias, sepes etc. frequens. Off.

3. A, Bardana (i illdenow): fol. cordat, pubeso 
centib. subtus tomentoso-incanis, capitul. paniculato-glomeratis, involucri arachnoideo-tomentosi foliol. margine setis acuminatis obsitis. S. I. 418. H. A. II. 36 .

A. 3-4' 24. Antecedentibus simile. In rudo ratis, pagis etc. Off.

334. 6ERRATURA.

Involucr. cylindric. imbricat. polyphyllum, fo. liol. inermib. v. apice appendice scariosa auctis. Receptac paleaceum, Papp, pilosus inae. qual. rigidus dentatus $r$. ciliatus.

1. S. tinctoria: fol, argute serratis basi pinnatifidis integrisve glabriusculis, capitul. corymbosis. S. I. 418 .

A. $2-3^{\prime} 2$. Fol, radicalia oblongo-ovata. Fl. 6-8. Cor. sanguineo: purpureae. In pratis, nemoribus subhumidis etc, Teg. Friedrf. Pa. peub. etc.

\section{CARDOƯ 3.}

Involucr. ventricos. imbricat. polyphyllum, fo. liol. simplicib. spina terminatis. Receptac, pa. leis setaceis obsitum. Papp. pilosus, radiis sin. plicib. $\nabla$. scabris $\nabla$. plumosis, basi in annu Ium connat. deciduis.

* Folïs decurrentibus, pappo scabro. 1. C. nutans: fol. interrupte decurrentib. spino 
sis, capitul. cernuis, involucri fol, lanceolat, su* perne patentibus. S. I. 419.

A. $3^{\prime \prime}-2^{\prime} \sigma^{7}$. Fol. sinuato-pinnatifida oblonga. Fl. 6-8. Cor. purpureae v. albae. In agris, ruderatis etc.

2. C. crispus: fol. decurrentib. oblong. sinuat. margine spinos, subtus lanuginosis, capitulis pedunculatis aggregat. terminalibus, involucri foliol. lineari-subulat. mucronat. patulis. L. IV. III. 1652. A. 2-3'. $\odot$. Fl. 7, s. Cor. pallide purpureae. In graminosis subhumentibus ad sepes etc. rarius.

$$
\text { * * Foliis clecurrentibus, pappo plumoso. }
$$

3. C. palustris: fol, decurrentib pimatifid. dentatis spinos. scabris, capitul. racemoso-glomeratis, involucri foliol, ovato-lanceolat. spinescenti-mucronat. adpressiz. Smith fl. br. 850 .

Cnicus palustris. L. IV. IIT. 16 กี2. Kth. 215.

A. 1-3 $3^{\prime}$. Fl. 6-9. Cor intense purpureae. In graminosis humidis, in agris, ad fossas etc. frequens.

4. C. lanceolatus: fol. decurrentib. supra hispid. subtus tomentos, pinnatifidis, laciniis bilob. divaricat. spinosis, involucri foliol, lanceolat. spinos. patentib. arachnoideo-pubescentibus. Sm. H.br. 847 .

Cnicus lanceolatus. L. W. III. 666. Kth. 215 . A. 3-4 ${ }^{\prime}$. Fl. 6-8. Cor, purpureae, inter- 
dum albae. In ruderatis, ad sepes, vias etc. frequens.

*** Foliis non decurrentibus, pappo plumoso.

5. C. arvensis (Curtis): fol. sessilib. lanceol, pinnatifido-dentat. undulat. spinos. glabr, subtus tomentosis, caule paniculato, involucri foliol. ovat. spinulos, apice patentibus. Sm. A. br. 850 .

Serratula arvensis. L. W. III.1646. Kth.214.

A. 2-4' 24. Fl, 6-9 Cor. dilute purpureae, rarins albae. In agris, ad sepes, vias etc. frequentissimus.

6. C. acaulis: fol. petiolatis pinnatifidis dentatis margine spinosis, caule v. pedunculo radicali sub. unifloro, involucri foliol. lanceol. arcte imbricatis, inferiorib spinosis. Sm. A. br. 855 .

Cnicus acaulis. L. W. III. 1681. Kth. 216.

A. 2-4" 24. Fl. 6-S. Cor. intense purpureae. In graminosis subhumidis. Friedifelde. Kalkb, Fasanerie etc.

7. C. oleraceus: fol. amplexicaulib. cordat. pinnatifid. ciliato-serrato-spinulosis, capitul. terminalib. subracemosis bracteat., bracteis subcolorat, ovatis, involucri foliol. lanceolat. spinosis. S. I. 422.

Cnicus oleraceus. L. W. III. 167s. K'th. 216.

A. 2-3' 4. Fl. 6-8. Cor. ochroleuca, rarius purpurea. In pratis humidis frequens. 


\section{ONOPORDON.}

Involucr. ventricosum imbricatum polyphyllum, foliol. spina simplici terninatis. Receptac. al. veolatum. Papp. pilosus, radiis basi in annulum connexis.

1. O. Acanthium: fol. decurrentib. ovato-oblong. sinuat, dentat. spinos, utrinque lannginosis, involucri foliol. patentissimis. S. I. 23 . H. A. VI. 44.

A. $\frac{1}{2}-6^{\prime} \sigma^{7}$. Fl. $6-8$. Cor. pallide purpurea. In solo meliore ad sepes, in ruderatis etc. froquens. Off.

337. CARIINA。

Involucr. ventricosum duplex, exterioris folioli laxi pinnato-spinosi, interioris scariosi colorati. Receptac. paleaceum, paleis apice multifidis. Papp. plumosus, radiis nonnullis inferne in laminam paleaceam connatis, omnib, basi in annulum connatis.

1. C. vulgaris: caule multifloro corymboso pubescente, fol. lanceolat. dentat, spinos. subtus pubescentibus. L. W. III. 1696 .

A. $\frac{1}{2}-1 \frac{T^{\prime}}{2} 0^{7}$. Fl. 7-9. Cor. Inteae cartila. gineae. In siccis graminosis, sylvis etc. fre. quens.

338. BYDENS.

Inroluer, duplex polyphyll. inaequale. Receptac: 
convexum paleaceum. Papp, aristae 2-6 ero ctae retrorsum aculeolatae.

1. B. tripartita: flor. discoideis, involucro exteriore flores superante, achaeniorum aristis erectis subternis, fol. tripartit. lanceol. dentatis. S. I. 424. A. $1-3^{\circ} \odot$. Fl, 7-10, Cor. flavae. In locis humidis, ad fossas etc. frequens,

2. B. cernua: flor, subradiantibus, capitul. cer nuis, involucro exteriore llor. superante, achaenio. rum arist. erect. subquaternis, fol. lanceol. subcordat. dentatis. S. I, 224 .

4. minima. B. vulgaris. \% coreopsis. A. $3^{\prime \prime}-1 \frac{1}{2}$ $\odot$ Fl. 7-10. Cor. Havae. Tota planta ex lutescenti viridis. In locis antecedentis copiose.

\section{EUPATORIUM.}

Involucr, oblongum imbricatum, foliol. inaequalibus. Receptac. nudum scrobiculatum. Styli longi profunde bifidi. Papp. sessilis pilosus $\nabla$. scaber.

1. E. cannabinuin: caule scabriusculo, fol petiolat. ternat. quinatisque, foliol. lanceol. serratis, intermedio longiore. H. A. VIII. 44 .

A. 3-5, 24. Fl. 6, 7. Flor corymbosi. Cor, roseo-vinosae. In solo humido, ad fossas, in pratss etc. 


\section{CHRYSOCOMA.}

Involucr. hemisphaerícum v. ovatum imbricatum, foliol. oblong. extus convexis. Receptac. nudun scrobiculatum a scrobicul, marginib. denticulat. scabrum. Styli vix corollis longiores. Papp pilosus sessilis, radiis denticulatis.

1. C. Linosyris: herbacea, fol. linearib. glabris, involucr, laxis, L. IV. III. 1791.

A. 1-2' 24. Fl. 6, 7. Flor. corymbosi. Cor. aureae. In collibus siccis fertilibus rarius. Schulzend, Crome, S. I. 425.

\section{ORDO II. SUPERFLUA.}

341. TAN A CETUM.

Involucr. hemisphaericum imbricatum, foliol. adpressis. Receptac. nudum. Papp. margo brevis membranaceus. (Flor. marginales obsolete trifidi.)

1. T. vulgare: fol. bipinnatifidis, laciniis serra• tis. L. W. III. 18i4. H. A. II. 4.

A. $3-5^{\prime} 4$. Flor. corymbosi aurei. Odor aromaticus. In agris, ad vias etc. Charlottb. Teg. etc. Off.

342. ARTEMISIA.

Involucr. ovatum v. suíglobosum imbricatum, foliol, adpressis, Receptac, nudum v. villosum. 
Papp. o. (Flor. marginales subulati edentuli $\$$, disci 5-dentati 후

* Receptaculum nudum (Artemisia.).

1. A. campestris: caule procumbente v. adscendente ramoso virgato, fol. caulin. setaceis pinnat. glabris, radicalib. pinnatis, laciniis 3 -fid. incanis, capitul. ovat. pedunculatis. H. A. II. 9. S. I. 426 .

A. 1-2 24. Fl. 8, 9. Cor. vinoso-roseae. Interdum tota planta rubet. In agris frequentissimum.

2. A. vulgaris: caule erecto ramoso, fol. subtus tomentosis, caulin. pinnatifidis, laciniis lanceolat. subdentat. acutis, floralib. indivis. linearib. lanceolatis, capitul. subsessilib. oblong. erectis, involucr. tomentosis. H. A. II. 12, S. I. 427.

A. 4-6, 4 . Cor. vinoso-roseae. In rudera. tis, pagis, ad sepes etc. frequens. * * Receptaculum villosum (Absinthium).

3. A. Absinthium: caule erecto ramoso, fol. incanis, radicalib, triplicato-pinnatifidis, laciniis lanceol. dentat. obtusis, caulin bipinnatifid. pinnatifidisve, laciniis lanceolat. acutiusculis, floralib indivis. lanceolatis, capitul, globos, pedunculat. nu. tantibus, H. A. II. 11. S. I. 427.

Absinthium vulgare. Kth. 219.

A. 3-4' 4. Cor. Iuteae. In solo argillaceo ad sepes, in ruderatis etc. linc inde. Off. 


\section{CNAPHAIIUM.}

Involucr. imbricatum, foliol. inaequalib. scarios; saepe coloratis. Receptac. nudum punctulatum v. scrobiculatum scabrum. Papp. pilosus scaber v. plumosus.

* Capitulis dioicis, pappi florum ơ radiis apice dilatatis (Antennaria).

1. G. dioicum: stoloniferum, caule simplici, fol. radicalib. spathulatis, corymbo simplici coarctato, capitul. dioicis, involucri foliol. interiorib. elongat. obtus. coloratis, II. A. Y. 6.

Antenraria dioica. Gaertner 1I. 410.

A. $\frac{\pi}{4}-\frac{\pi}{2} \cdot 24$ FI. 5, 6. Cor. niveae $\nabla$. roseae. In locis siccioribus pratorum, nemorum, collium frequens.

- Capitulorum floribus omnibus જ̧ (Gnaphalium)

2. G. arenarium: herbaceum, fol. incano-lanatotomentosis, radicalib. spaihulato-lanceolat. obtusis, caulin. lineari-lanceolat, acutiusculis, cory mbo composito. H. A. V. 5.

A. $\frac{1}{4}-1 \cdot 2$ Fl. 6-10. Cor. sulphnieae $\nabla$. subaurantiae. In solo arenoso copiosissimum.

** Capitulorum floribus mediis paucis ఫ̧, marginalibus pluribus $q$ (Filago).

3. G. rectum (Smith): caule herbaceo erecto simplici, fol lineari-lanceolat, subtus sericeis, capi- 
ul, terminalib. et axillarib. sessilibus spicatis. L. IV. III. 1894 .

Gnaphalium sylvaticum. W. p. n. s14.

A. $\frac{1}{2}-1 \frac{1}{2} \cdot 4$. F1. 6, 7. Flor. spicati. Involucri foliola basi viridia, medio fusca. In graminosis sylvarum et inter frutices. Jungfh. Grunw. Thierg. etc.

4. G. luteo-album: herbaceum, fol. semiamplexicaulib. lineari-lanceolat. subrepand. utrinque lanuginoso-pubescentibus, inferiorib. obtusis, superiorib, acutis, corymbo glomerato. L. WV.III. 1871.

A. $\frac{1}{2}-1^{\prime} \odot$. Fl. 7, 8. Involucri foliola exteriora viridicantia, interiora pallide lutea. In solo humido circum lacus. WVeifss. Temph.

5. G. uliginosum: caule herbaceo ramoso diffuso lanato, fol. lineari-lanceol. utrinque angustat. to. mentosis v. glabris, capitul. terminalib. conglomeratis, glomerul. foliosis. S. I. 430.

A. $\frac{\mathrm{I}}{2}-1$ ' $\odot$. Fl. 7, 8. Involucri foliola exteriora lanata, interiora sordide fuscescenti-lu. tea. In locis humidis ad fossas etc.

6. G. germanicum (Hudson): herbaceum, caule erecto dichotomo, fol. lineari.lanceolat. tomentos. acutis, capitul. globosis alarib. terminalibusque. L. W. III. 1894.

Filago germanica $L$.

A. 3-18" $\odot$. Fol. undulata. Fl. 7, 8. InvoF. B. 
lucri foliola inferiora lanata, superiora scario. sa sordide lutea. In agris post messem his illisve frequens. Willmersd. Hasenh. WVeilss. 7. G. arvense: herbaceum, caule erecto paniculato, fol, oblongo-lanceolat. lanuginosis, capitul. congest. lateralib. terminalibusque tomentosis. L. W. III. 1897 .

A. $\frac{\mathbf{r}}{4}-2^{\prime} \odot$. Fol. erecta. Fl. 7, 8. Involucri foliola lanuginosa. In arvis et collibus arenosis frequens.

8. G. montanum: herbaceum, caule erecto subdichotomo, fol. lineari-lanceolat. adpress. tomentosis, capitul. confert. alarib. terminalibusque. L. Wr. III. 1896.

Filago montana.

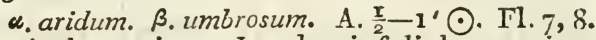
Capitula conica. Involucri foliola exteriora tomentosa, interiora Intescentia. Fl. જ̧ in quo. vis capitulo $1-3$. In solo sterili arenoso frequens.

\section{ERIGERON.}

Involucr. cylindricum $v$, hemisphaericum imbricatum, foliol. angust. apice patulis. Receptac. nudum, dein scrobiculatum. Papp, sessilis pilosus.

1. E. canadense: caule capitulisque paniculatis hirtis, foliol. Ianceolat. ( $v$. Jineari-lanceol.) cilia. tis. L. W. III, 1954. 
A. $2-3^{\circ} \odot$. Fl. 6-9. Color totius plantae pallide viridis. In agris arenosis et solimelio. ris undique.

2. E. acre: caule ramoso hirto, capitul, corymboso-paniculat, subsolitariis hirtis, fol, lanceolato. ligulat. ciliatis. H. A. III. 30 .

A. $\frac{\mathrm{r}}{2}-1 \frac{\mathrm{x}}{2}, 2$. Fl. 7, 8. Cor. roseae. Tota planta saepe purpurascit. In solo arenoso apri. co frequens.

545. TUSSIIAOO.

Involucr. polyphyllum, foliol. aequalib. submembranaceis simplici serie dispositis. Receptac. nudum scrobiculatum v. elevato-punctatum. Papp. sessilis pilosus. (Flor. sacpe polygami.) - Capitulis solitariis terminalibus (Farfara).

1. T. Farfara: scapo 1.cephalo subnudo bracteato, flor. radiantibus, fol. cordat. angulat, dentat. subtus tomentosis. II. A. II. 16 .

A. $\frac{x}{4}-\frac{x}{2} 24$. Pad. repens. FI. 4. ante fol. cruptionem. Cor. aurene. In solo argillaceo et calcareo passim. Spand, Willmersd. WV, Heinersd. S. Rüdersd.

* Capitulis in thyrsum collectis (Petasites).

2. T. Petasites: thyrso oblongo, flor, discoideis, fol. oblongo-cordat. inaequaliter denticulat, subtus 
pubescentibus, lobis approximatis. L. W.III. 1970. H. A. II. 17.

a. hermaphrodita. 6. foemina. A. $\frac{1}{4}-1,24$. Fl. 4, 5. ante foliorum eruptionem. Capitula et scapi purpurascunt. Cor.purpureae. In solo humido ad fossas etc. rarissima. Schönh. W. Spand. W. Thierg. IV.

3. S. spuria (Retz): thyrso oblongo, flor, discoideis, fol. oblongo-cordat. inaequaliter denticulatis, utrinque niveo-tomentosis, lob. divaricat. trilobis. L. W. III. 1772 .

A. $\frac{\pi}{4}-\frac{3}{4} \cdot 24 . \mathrm{Fl}, 4$ antequam fol. apparent. Scapi lutescentes. Cor. luteae. In ripis areno. sis fluminum. Pichelswerder.

\section{SExECro.}

Involucr. cylindricum duplex, exterius brevius foliol. imbricatis, interius longius, foliol. aequalib. apice sphacelatis duplici serie dispositis Recept. scrobiculatum mamillatum. Papp. sessilis pilosus.

* Corollis o radiantibus (Senecio).

1. S. vulgaris: fol. amplexicaulibus pinnatifidis dentatis, corymb. terminalibus paucifloris, capitul. discoideis coarctat. plerumque cernuis. H. A. VIII. 10.

A. $\frac{T}{2}-2^{\prime}$. Fl. 5-10. Cor. citrina. In agris, ruderatis, ad sepes etc. undique. 
* Corollis marginalib. radiantibus ( $f a$ cobaea).

+ Radio revoluto.

2. S. viscosus: fol. pinnatifid. viscidis, involucri externi laxi foliol. internum subaequantibus, cor. radiantibus, radio revoluto. S. I. 435.

A. 1-1 $\frac{1}{2}^{\prime}$. T1. 7-9. Cor. Havae. In siccis sterilibus, ruderatis etc. hinc inde. Kalkbge. Teg. etc.

3. S. sylvaticus: fol. subpinnatifid, denticulat. subviscidis, involucri exterioris adpressi foliol. brevissimis, cor. radiantibus, radio revoluto. S.I. 436 . Antecedenti affinis. $\odot$. Fl. 6-9. Cor. pallidiores. In sylvis nemoribus et locis siccis apertis frequens.

$$
\text { † Radio patente. }
$$

4. S. Facobaea: caule erecio, fol. sublyrato-bipinnatilid. dentat. radicalibus obovatis integris, corradiantibus, radio patente. S. I. 436.

Senecio aquaticus. L. W. III. ${ }^{1797}$.

a. campestris. B. palustris. A. $\frac{\pi}{2}-1 \frac{\pi}{2}$ 2 4 . Fl. 7-9. Cor. flavae. In solo argilloso sicciore et humido, in pratis etc. frequens.

$t+\dagger$ Radio patente, foliis integris.

5. S. paludosus : canle stricto, fol. semiamplexicau. lib. lanceolat. argute serrat. subtus subvillosis, co. 
r ymbo terminali divaricato, cor. radiantibus, radio patente. S. I. 437.

A. 2-5'24. Fl. 6, 7. Cor. aureae. In pratis et paludibus haud rarus. Jungfh. Papent. etc.

\section{ASTER.}

Involucr. imbricatum subsquarrosum, foliol. infimis patentibus. Receptac. nudum punctulat. glabr. v. scrobiculatum scabrum. Papp. sessilis pilosus. (Flor. disci radiantes plures quam 10, albi, lilacini v. violacei.)

1. A. Amellus: fol. oblongo-lanceolat. integerrim. scabris, ram. corymbosis, involucro imbricato subsquarroso, foliol. obtusis, interiorib. mcmbranaceis apice coloratis. L. W. III. 203I.

A. 1-1 $\frac{1}{2} \cdot 2$. Fl. 7, 8. Flor corymbosi. Cor. radii violascenti-coeruleae. In collibus siccis. Kalkb,

\section{SOLIDAGO.}

Involucr. imbricatum, foliol. infin. conniventibus. Receptac. nud. scrobiculatum. Papp. ses. sílis pilosus. (Flor. disci radiantes $5 \mathrm{v}$. paulo plures, omnes lutei.)

1. S. Virgaurea: caule erecto tereti superne ramoso pubescente, fol. serrat. subglabris, inferiorib. oblongo-ovalibus, superiorib. lanceolatis, racem. erectis, cor, radiantib, elongatis, H. A. VIII. 437. 
A. $\frac{\mathrm{r}}{2}-3^{\prime}$. Fl. 7-9. Cor. anreae. In locis graminosis sylvarum et collium frequens. Grunw. Friedrf. etc.

349. CINERARIA.

Involucr. polyphyll. simplex, foliol, aequalibus. Receptac. nudum scrobiculatum. Papp. sessilis pilosus.

x. C. palustris: caule erecto villoso superne corymboso, fol. lato.lanceol. dentato-sinuat. pubescentibus. S. I. 438 .

A. $\frac{\mathrm{T}}{4}-^{\prime} \sigma^{7}$. Fl. 6, 7. Cor. aureae. In locis paludosis et turfosis linc inde, Grunw. Tals. dorf etc.

\section{I N U L A.}

Involucr. imbricat. polyphyllum subsquarrosum, foliol. inferioribus patulis. Antherae in setas duas desinentes. Receptac. nudum. Papp. simplex.

3. I. britannica: caule corymboso villoso, ramis lateralib. apice longioribus, fol. amplexicaulibus lanceolat. basi serratis, subtus pilosis. L. IV. III. 2090.

A. $1-3^{\prime}$ 24. Tl. 7-10. Cor. aureae radiis patentissimis. Involucri foliola lineari-lanceo. fata. In solo subhumido ad margines pratorum, ad vias etc.

2. I. dysenterica: caule paniculato lanuginoso, 
ram. lateralib. apice longiorib patulis, fol. amplezicaulib. cordato-oblong. serrat. subtus pilosis, involucri foliol. setaceis. H. A. VI. 46 .

A. $1-2$ ' 4. Fl. 7,8 . Cor flavae. Radii breves. In locis subhumidis inter frutices et ad fossas rarior. Vor d. Köpu, u. Hallisch. Th. W. Spand. S. Schönh.

3. I. salicina: caule paniculato glabro erecto, ram. angulatis inferiorib. altioribus, fol. lanceolat. recurv, serrato-scabris, involucri foliol. lanceolatis. S. I. 440.

A. 1-2, 4. Fl. 7, 8. Cor. Havae. In pratis humidis, ad fossas, in pascuis etc linc inde. Schönb. Jungft. WV. Thierg. Kth Grunw.

4. I. Pulicaria: caule ramosissimo paniculato hirto, ramis mediis longioribus, fol. amplexicau. lib. oblong. undulat. villosis, involucri foliol. lineari-setaceis. Kth. 227.

A. $\frac{1}{2}-1^{\prime} \odot$. F1. 7-9. Flor. subglobosi, radio minutissimo. Cor. Havae. In graminosis subhumidis ad sepes, vias etc. hinc inde. Spand. Heinersd, S. Niariend.

351. BEIII8.

Involucr. hemisphaeric. polyphyllum, foliol biserialibus aequalibus. Receptac. conicum nudum. Papp. o. 
3. B. perennis: scapo nudo sinıplicissimo, fol. obovat. crenat. subtrinerviis. H. A. III. "2́.

Fl. 4-10. Cor. albae v. purpurascentes 4 . In locis humidis undique.

352. CHRYSA NTEMUN.

Involucr. hemisphaeric, imbricatum, foliol. nargine scariosis. Receptuc. convexum nudum. Papp. o.

1. C. Leucanthemum: caule erecto subramoso, fol. caulin. amplexicaulib. lanceol. serrat, basi inciso-dentatis, radicalib. obovat. $\nabla$. subspathulatis serratis petiolatis. S. I. 4,1 .

A. 1-2' 24. Fl. 6, 7. Cor radiantes niveae. In solo humido e. c. in pratis frequens.

2. C. segetum: caule erecto ramoso, fol amplexicaulib. glaucis superne laciniat. basi dentatis. $S$. I. 441 .

A. 1-2' $\odot$. Fl. 6-9. Cor. Alavae. Inter segetes rarissimum. Grunw. W.

\section{PYRETHRUM.}

Involucr. hemisphaericum imbricatum, foliolis margine scariosis. Receptac. nudum convexum. Papp. margo prominulus.

I. P. Parthenium (Smith): caule ramoso erecto, fol. pinnatis, pinn. oblong. obtus, pinnatifid. inciso-serratis, superiorib. conlluentibus, capitul. co- 
rymbosis, radio involucro fere duplo longiore, pappo dentato. L. W. III 2155. H. A. VI 20.

A. $1-2,2$. Fl. 6-9. Cor. radii albi, disci Iutei (Odor aromaticus.) In ruderatis, ad pagos linc inde. Spand. S. Teg.

2. $P$. inodorum (Smith): caule ramoso erecto, fol. subtripinnatifidis, laciniis linearibus acutis, capitul. terminalib. solitariis, radio involucro fere triplo longiore, pappo integerrimo. S. I. 442.

Chrysanthemum inodorum. H. A. I. 4 .

A. $\frac{\pi}{2}-1 ' \odot$. Fl. 6, 7. Flor. radiantes apice 3-crenati albi. Herba inodora. In ruderatis, agris et arvis passim. Spandau.

354. MATRICARIA.

Involucr. imbricatum, foliol. herbaceis. Receptac. nudum conicum intus cavum. Papp. o.

1. M. Chamomilla: fol, subbipinnatifidis, laciniis linearibus, receptaculo cavo conico, involucri foliol. obtusiusculis, S. I. 443 . H. A. I. 3.

A. $\frac{r}{2}-2^{\prime} \odot$. Fl, 6, 7. Corollae radii albi pose florem reflexi. Odor aromaticus proprius. Planta glabra. In agris inter segetes et in arvis frequens. Off.

355. ANTHEMIS.

Involucr. hemisphericum imbricatum, foliol. magis minusve scariosis. Receptac. convexum $\nabla$. 
conicum paleaceum. Papp. o. v. margo membranaceus.

\section{* Radio albo (Chamaemelum).}

1. A. arvensis: receptac, inani conico, paleis cymbiformibus, fol. subbipinnatifid. pubescentibus, la cinijs lanceolatis. H. A. I. 5 .

A. $1-1 \frac{\pi}{2} \cdot \odot$. F1. $6-8$. Planta tota pilis cam rescens. In agris, ad vias, ruderat. etc, frequens.

2. A. Cotula: receptac. inani conico, paleis setaceis, fol. subtripinnatifid. glabris, laciniis lanceolatis. H. A. I. 6.

A. $1-3^{\prime} \odot$. Fl.6-8. Odor ingratus. Planta glabra. In ruderatis, pagis etc. passim frequens. Friedrf. Weifss. Spand. etc.

$$
\text { - Radio luteo (Anthemis). }
$$

1. A. tinctoria: receptac. convexo, paleis linearib. apice subulatis, fol. stabbipinnatifid. serratis subtomemtosis, laciniis lanceolatis. S. I. 444 .

A. $1-2^{\prime} 24$. Fl. 7, 8. Planta canescens. In solo calcareo vo argillaceo hinc inde. Rüdersd.

$$
\text { 356. ACHILIEA. }
$$

Involucr, ovat, imbricatum, foliol. adpressis. Receptac. planum paleacenm. Papp. 0.

1. A. Ptarmica: fol. linearib. acuminatis subaequaliter argute serrulat. glabris, caule paniculato, corymb, compositis, paleis integris. Ktb, $23^{\circ}$. 
A. $2-3^{\prime} 2 \nmid$. Rad. repens. Cor. albae. In Io. cis subhumidis graminosis, in pratis, fruticetis etc. OIF.

2. A. Millefolium: fol bipinnatifidis pilosis, laciniis linearibus dentat. mucronatis, caule sulcato. S. I. 445 .

a. dilatata. B. contracta. A. $\frac{\mathbf{r}}{4}-3^{\prime}$. Fl. 6-10. Cor. albae v. purpurascentes. Odor proprius. In solo humido et sicco undique. Off.

357. CAEINGSOGEA.

Involucr. hemisphaeric. imbricat, polyphyllum. Receptac. paleaceum. Papp. ex plurib. squamis paleaceis compositus.

1. G. parviflora (Cavanilles): fol. ovat. triplinervis serratis. L. W. III. 228 .

A. $\frac{7}{2}-1^{\prime} \odot$. Fl. $7-10$. Cor. Havescentes. In agris oleraeeis et ad fimeta passin. Zwischen Stadt u. Schöneb.

\section{ORDO III. FRUSTRANEA.}

358. C ENTAUEA.

Involucr. imbricatum, foliol, apice lacer. ciliat. v. spinosis. Receptac, paleaceo-setosum. Рapp. setaceus $\nabla$. paleaceus inaequalis centro umbo. nato vario.

* Foliolis involucralibus ciliato-serratis (Cyanus). 
1. C. Cyanus: caule subcorymboso, fol. linearib. sessilib. integerrimis, infinı. dentat. $\checkmark$ subpinnatifidis, foliol. involucralib. ciliatis serratis adpressis. II. A. VII. 32. S. I. 447.

A. $1-2^{\prime} \odot$. Fl. $6-10$. Cor. cyaneae rarius albidae In agris inter segetes et arvis frequentissima.

2. C. paniculata: caule paniculato, fol. inferiorib. bipinnatifidis, superiorib, pinnatifidis, involucri foliol. osat. ciliat. adpressis. L. W. III. 2292.

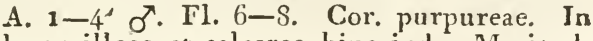
solo argilloso et calcareo hinc inde. Mariend. Talsd. etc.

3. C. Scabiosa: caule subsimplici, fol pinnatifidis, laciniis lanceolat. acut. basi pinnatifidis, involucri foliol. ovat. fimbriato-ciliat. pubescentibus. L. W. III. 2296. HI, A. VII. 33 .

A. $1 \frac{\mathrm{T}}{2}-2 \cdot 24$. Fl. $6-9$. Cor. purpureae. In agris inter segetes, ad vias etc. frequens.

* Foliolis involucralibus aridis scariosis, integerrimis $\because$. laceris (Rhaponticum).

4. C. Facea: caule corymboso, ram. angulatis, fol. Ianceolat. integerrimis, radicalib. subdentatis, involucri foliol. scarios. ovat. apice laceris. S. I. 448 .

A. $\frac{\tau}{4}-2^{\prime}$. Fl. $6-9$. Cor. purpureae. In solo humido et sicciore. 


\section{ORDO IV. NECESSARIA.}

359. CAIENDUIA.

Involucr. simplex polyphyllum aequale. Receptac. planum nudum. Papp. o. Achaen. difformia, disci membranacea.

1. C. arvensis: achaen. cymbiformib. muricat. incurratis, exteriorib. lanceolato-subulat, dorso cristato $\cdot$ muricatis. L. W. II. 2339 .

A. $\frac{r}{4}-\frac{x}{2}, \odot$. Fol. cordato-lanceolata alterna sessilia Fl. 6-8. Cor. flavae. Inter segetes rarissima. IV.

\section{I, XIX, GYNANDRIA. \\ ORDO I. MONANDRIA.}

360. OF CHIS.

Cor. 5-petala, petalo superiore fornicato. Labellum basi subtus calcaratum. Anthera terminalis adnata.

* Glandulae $(1-2)$ pedicellorum pollinis inclusae cucullo unico (Orchis R. Brown).

1. O. Morio: labello trilobn, lob. crenat. obtusis, medio emarginato, petal. adscendentib. obtusis, cornu conico adscendente germine breviore. I. W, IV. 18 . 
A. $\frac{7}{2}-1 \cdot 24$. Bulbi 2 globosi. Fol. lanceolata. Fl. 5. Cor. purpurea. In pratis humidis hinc inde. Temph. Frankf. Th. Friedrf. etc. Off.

2. O. militaris: labello tripartito punctato scabro, laciniis lateralib. linearibus, intermedia biloba obtusa cum mucrone interjecto, petal. acut. conniventibus, cornu recto germine duplo breviore, bracteis obsoletis. L. IV. IV. 22.

A. $\frac{x}{2}-1 \frac{x}{2} / 24$. Bulbi 2 ovati. Fol. oblongo. lanceolata. F1.5. Cor. dilutepurpurea. In pratis Iumidis frequens. Friedrf. Charlottb, etc. Off.

3. O. latifolia: Iabello leviter trilobo Iaterib. re

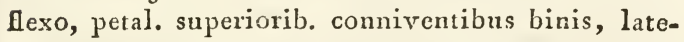
ralibus reflexis, cormu conico germine breviore, bracteis flore longioribus. L. WV.IV. $2 \mathrm{~S}$.

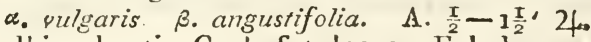
Bulbi palmati. Caul. fistulosus. Fol. lanceo. lata maculata v. non maculata. Fl. 5. Cor. purpureae, dilute purpureae et albae. In pratis humidis undique $\beta$. Grunw.

4. O. [maculata: labello plano trilobo crenato, petal. superiorib. conniventibusi, lateralib. patentibus, cornu cylindraceo germine breviore, bracteis longitudine germinis. L. W. IV. 31 .

A. 1-1 1 . 4 . Bulbi palmati. Fl. 5, 6. Habitus gracilior, quam in antecedente. Caul. 
solidus. FI. 5, 6. Cor. pallidiores. In pratis humidis. Heiligens, Charlotıb. Fricdrf. etc.

* Glandulae pedicellorum pollinis nuda approximatae. (Gymnadenia R. Brown.)

5. O. conopsea: labello obtuse trilobo, petal. lateralib. patentissimis, cornu subulato germine duplo longiore. L. WV. III. 32.

Gymnadenia conopsea. S. I. 451.

A. $1-1 \frac{1}{2} /$ \& Bulbi palmati. Fol. lanceolata v. lineari-lanceolata. FI. 5, 6. Cor purpurea. In pratis humidiusculis. Frankf. Thor. Heiligens. Spand. etc.

* * Glandulae pollinis nudae distinctae. (Habenaria $R$ Brown.)

6. O. bifolia: labello lineari integerrimo obtuso, cornu germine duplo longiore, fol. caulin. vaginantib. brevissimis, radicalib. bin. oblong. basi attenuatis. L. W. IV. 10.

Habenaria bifolia S. I 452.

A. 1-2, 4 Bulbi ovales. Fl, 5: Cor. fragrantissima, alba. In sylvis frondosis humidis hinc inde. Papenb. Cöpn. Heide etc.

\section{OPHRYs.}

Cor. 5-petala subringens, petal. patentibus. $L a-$ bellum e basi styli ecalcaratum patens. Anthera terminalis adnata.

1. O. Monorchis: scapo nudo, labello tripartito, 
laciniis linearibus, lateralib. divaricatis, bracteis longitudine germinis. L. W. IV. 61 .

Herminium Monorchis ( $R$, Brown) S, I. 452.

A. $\frac{1}{4}-\frac{1}{2}{ }^{\prime} 2$. Bulbi ovales. Fol. ovato-Janceolata vaginantia. Fl. 5, Cor. lutescenti-vi. rides. Glandulae pollinis nudae distinctae. In pratis humidis, uliginosis. Steglitz, Starckloff. Friedrf. Frankf. Th, frequens.

\section{COODYERA.}

Cor. ringens, petal. exteriorib. anticis labello inferne gibboso superne indiviso suppositis. Co. lumna libera. (Pollen angulatum.)

1. G. repens (R. Brown): fol. radicalib. ovatis, labello petalisque lanceolatis Ait. Kew. V. 198. Neottia repens. L. W. IV. 75. Satyrium repens $L$. A. $\frac{1}{4}-\frac{3}{4} \cdot 4$. Rad. repens. Fl. 7,8. Flor. secundi. Cor. albae. In sylvis muscosis rarius. Teg.

363. NEOTTIA.

Cor. ringens, petal. exteriorib. anticis labello imberbi suppositis, interioribus conniventibus. Columna aptera. (Pollen farinaceum.)

1. N. spiralis (Swartz): fol, radicalib. oblong. subpetiolatis, spica tortili, flor. secundis, labio ovato. L. W. IV. 73 .

Ophrys spiralis L.

F. B. 
A. $\frac{T}{8}-\frac{1}{4} \cdot 2$. Bulbi ovati oblongi. Fl. 6,7 Cor. albae suaveolentes. In pratis rarius. Rummelsb. W. Starckloff

Chamisso Adnotat, ad Kunth 12, et Schlech. tendal I. 453 censent: hanc plantam errore quodam hic enumeratam esse; sed post Willdenowium 1816 pauca specimina invenit Starckloff, qui benevole unum nobis tribuit.

$$
\text { 364. I ISTERA. }
$$

Cor. irregularis. Labellum bilobum. Columna aptera. Anthera basi inserta. (Pollen farinaceum.) 1. L. ovata ( $R$. Brown) " caule bifolio, fol. ovat. oppositis, columna postice cucullo antherae incumbente aucta. Ait. Kew. 5. 501 .

Epipactis ovata. L. W. IV. 87. Kth. 237. Ophrys ovata. $L$.

A. $\frac{\pi}{2}-2^{\prime} 24$. Rad. fibrosa. Fol. petiolata. Fl. 5, 6. Cor. Alavo-virescentes. In sylvis humidis frondosis frequens. Thierg. Papenb. etc. 365. NEOTTID IUM.

Cor. irregularis. Labellum bilobum. Columna postice appendice mobili antheriformi (anthera altera?). Antherae basi insertae prius incumbenti. (Pollen farinaceum.)

1. N. Nidus avis: caule viginato aphyllo. S.I.454. Ophrys Nidus avis L. Epipactis N. a. L.W.IV, 87. Kth. 236. 
A. $\frac{T}{2}-I^{\prime} 2$. Rad. fibrosa, fibris cylindricis. Fl. 6. Cor. fusca. In sylvis frondosis hinc in. de, Grunw. Kth. Briselang S. Papenb.

366. EPIP A c т Is.

Cor. 5-petala. Labellum inferne ventricosum su. perne v. indivisum v. 3-lobum, lobo medio majore articulatim connexo: (Pollen farinaceum.)

1. 1. E. latifolia (Swartz): fol, ovat. amplexicaulibus, bracteis inferiorib. Hore longioribus, florib. pendulis, labello integerrimo acuminato petal. breviore, germinib. pubescentibus. L. W. IV. 83 . Serapias latifolia.

A. $\frac{1}{2}-3^{\circ}$ 4. Flor. racemosi. Fl. 6, 7\% Cor. ex viridi et fusco purpurascens. In sylvis sub. lumidis umbrosis. Cöpı. Heide, Papenb. etc.

2. E. palustris (Swartz): fol. lanceolat. amplexicaulibus, bracteis flore brevioribus, florib. pendulis, labello crenato obtuso petalis aequali, germi. nib. pubescentibus. L. IV. IV. \$4.

Serapias longifolia. L.

A. $1-1 \frac{1}{2} \cdot 2$. Rad antecedentis. Fl.5,6. Cor. petala interiora alba picturis purpureis. In pratis humidis frequens. Grunw. Friedrf. etc.

3. E. rubra: fol. lanceolatis, bracteis germine longioribus, flor. erectis, labello acuto lineis clavatis undulatis, germinib. pubescentibus. S. I. 456.

Serapias rubra. L. 
A. 1-2' 24. Fl. 6, 7: Flor. pauci, racemosi. Labellum trilobum, lineis lavis. Cor. maxima, roseo-purpurea. In sylvis frondosis hinc inde rarius, Papenb. S. Teg. Cöpn. Heide.

$$
\text { 367. } \mathrm{I} \wedge \mathrm{x} \wedge \mathrm{x} \mathrm{Is} \text {. }
$$

Cor. petala 5 angustiora patula v. deflexa. Labellum explanatum indivisum sessile (saepius posticum.). [Massae pollinis 4 parallelae apicibus stigmati affixae.]

I. M. Loeselii: fol. binis ovato-lanceolatis, caule trigono, labello apice ovato recurvato. L. W.IV.92. A. $\frac{\pi}{4}-\frac{1}{2} \cdot 2$. Bulbus ovatus humo superinsidens ad basin insequentis anni bulbum format. Fl. 6, 7. Cor. viridi-lutescentes. In locis turfosis. Grunw.

2. $M$. paludosa: fol. subquaternis apice scabris, caule pentagono, labello concavo acuto. L.W. IV. 91 .

Ophrys paludosa. L.

Habitus gracilior. Partes minores 24. Fl. 6, 7 In paludibus turfosis. Machenow. Müggelsb. Chamisso. Grunw.

\section{ORDO II. IIEXANDRIA. 368. ARISTOLOCHIA.}

Cal. o. Cor. 1-petala ligulata basi ventricosa. Caps. inf. 6.locul. polysperma. Sem. angulo interno loculamentorum affixa. 
1. A. Clematitis: caule erecto, fol, subrotundocordat. obtusiuscnl. petiolatis, peduncul. uniflor. aggregatis, cor. erectis, labio oblongo. L. W. IV. 163. H. A. IX. 23.

A. $\frac{x}{2}-2 ' 24$. Rad. repens. Fl. 6, 7. Flor. axil. lares. Cor, Hava. Ad sepes hinc inde. Spandau,

\section{1. XX. MONOECIA. \\ ORDO I. MONANDRIA.}

369. ZANNICHRLIIA.

Flor. $\sigma^{\top}$ et $Q$ in eadem spatha. Flor. $\sigma^{\top} \mathrm{Cal}$. 0 . Cor. o. Stamen 1. Flor \& Cal. simplex campa. nulatus, Pistilla $3-5 \cdot$, stigmatibus peltatis. Achaen $3--5$.

1. Z. palustris: anthera 4-loculari, stigmatib. in. integerrimis, achaen. dorso denticulatis. L. W. IV. 181.

Fol. linearia $\odot$. Fl. 7. Cal. albidus, serius fuscus. Habitus Potamogetonis, In fossis et lacubus. Teg. Kth.

\section{ORDO II. TRIANDRIA.}

\section{0, T Y PHA.}

Flor. spicati $\sigma^{\pi}$ supra ㅇ. Flor. $\sigma^{\pi}$ Cal. simplex obsoletus 3-phyllus, Flor. \& Cal. et cor. 0. 
Achaen. stylo persistente coronatum, pedicello filiformi, a basi ad medium pilis longissimis basi angulo flexis obsito, impositum.

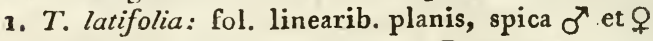
approximatis, utraque cylindrica. L. W. IV. 197.

A. 3-5, 24. Fl. 7, 8. Spica $\sigma^{7}$ Alava marcescens, $Q$ intense fusca crassa. Antherae $1-4$ in filamento communi. In paludibus et fossis profundis frequens. Teg. Jungfh. etc.

2. T. angustifolia: fol. linearibus canaliculatis, spica $\sigma$ femineaque remotis, utraque cylindracea. L. W. IV. 198 .

Habitus minor et tenuior. 24. Fl. 7,8. Loci similes.

371. SPAROANIUM.

Flor. glomerati $\sigma^{\top}$ supra . F. Flor. $\sigma^{\top}$ Cal. simplex 3-phyllus. Flor. ㅇ Cal. simplex 3-phyllus, Achaen, drupaceum siccum.

1. S. ramosum (Smith): fol. basi triquetris, late. rib. concavis, pedunculo communi ramoso, stigm. lineari. L. W. IV. 199.

Sparganium erectum. $\propto_{0} \mathcal{L}$.

A. 2-4' 4. Rad. repens. Caulis paniculatus. Fl. 5, 6. In locis aquosis, paludibus, fossis etc. frequens.

2. S. simplex (Smith): fol. basi triquetris, Jate- 
rib. planis, pedunculo communi simplici, stigm, lineari. L. W. IV. 199.

Sparganium erectum. $\beta$. L.

Habitus prioris, sed gracilior. 4 . Iisdem locis.

3. S. natans: fol. decumbentib. planis, pedunculo communi simplici, stigm. ovato brevissimo, capitulo masculo subsolitario. L. W. IV. 200.

A. 1-1 $\frac{1}{2}{ }^{\prime}$ 2. Tl. 6. Capitula subterna. In aquis et paludibus frequens. Grunw. Talsd. Jungfh. etc.

\section{CAREX.}

Flor. spicati varie dispositi. Flor. ơ Cal. sim. plex gluma. Flor. 우 Cal. gluma solitaria. Cor. 1-phylla ventricosa, ore pervio. Stigm. $2-3$. Achaen. 3-quetra cor. persistente inclusa.

a) Spica terminali simplici.

1. C. dioica: spica simplici dioica, stigm. binis, fructib, erectiuscul, ovat. nervosis superne margine hispidis, fol. culmisque glabris. L. W. IV. 407. A. 3-8' 24. Rad. repens. Fl. 5, 6. In solo paludoso turfoso hinc inde. Grunw. Jungfh. Friedrf. etc.

b) Spica e spicis androgynis alternis sessilibus composita.

2. C. chordorhiza: spic. androgyn. superne mase. in formam ovatam aggregatis, stigm. 2, fructib. 
orat. acuminatis, fol. culmeis adpressis culmo basi subramoso, L. W. IV. 219 .

A. $\frac{3}{4}-1 \cdot 2$. Rad. repens. Culmi adscendentes laeves. Fol. culmo breviora plana margine scabra. F1. 5, 6. In paludibus turfosis udis. Jungfh. S.

3. C. arenaria: spica androgyna composita, spicul. altern. confertis superiorib. masculis, inferiorib. 오, intermediis apice $\sigma^{7}$, stigm. 2, fructib. marginat. ovat. bifid. ciliato-serratis, culmo incurro. I. W. IV. 223. H. A. V. 7.

A. $\frac{1}{2}-1,2$. Rad, repens et per intervalla culmos protrudens. Fol. plana margine scabra. Culmi trigoni. Fl. 5-6. Glumae ovato-lanceo. latae. In solo arenoso frequens. Off.

4. C. intermedia (Goodenough): spica androgyna composita, spicul. altern. confertis, inferiorib re. motiusculis, superiorib. et inferiorib. foemineis, intermediis masc., stigm. 2, fructib. ovat. marginat. bifidis ciliato-serratis, culmo erecto. L. W. IV. 224. H. A. V. 8.

A. $\frac{\pi}{2}-2 \cdot 4$. Fl. 5, 6. In solo humido paludoso frequens.

5. C. Schreberi (Willdenow): spica androgyna composita, spicul. ovat. altern. confertis inferne masculis, stigm. 2, fructib. ovat. bidentatis. L. W.IV. 225. A. $\frac{3}{2}=1 \frac{r}{2} \cdot 2$. Fl. 5. Fructus non margind- 
tus. Affinis C. arenariae et intermediae. In gra. minosis siccis camporum et sylvarum. Jungfb. Spand. Hasenh. Grunw. etc.

6. C. schoenoides (Host): spica androgsna composita, spicul. oblong. altern. confert. superne masculis, stigm. 2, fructib. subrotundo-orat. marginat. bidentatis, glum. oblong. acut, submueronatis. L. IV. IV, 226.

In graminosis. 24. Fl.? Rebentisch.

7. C. ovalis (Gooden.): ispica androgyna composita, spicul. subsenis subrotundo-elliptic, alternis subapproximatis, inferne mascul., stigm. 2, fructil ovat. marginat. bidentat, ciliato-serratis. L. W. IV. 229 .

A. $\frac{3}{4}-1 \cdot 24$. Rad. caespitosa, fibrosa. Culm: 3-goni. Fol plana margine scabro. In solo humido, in pratis, ad fossas haud rarus. Thierg. Jungfh. Heiligens. etc.

8. C. vulpina: spica androgyna composita, spicul. numerosis ternatim quinatimve aggregat. ob. long. confert. superne mascul., stigm. 2, fructib. ovat. 2-dentatis compresso-triquetris patentibus, bracteis setaceis. L. W. IV. 231 .

A. $1-2^{\prime}$ 2. Rad. caespitosa. Culmi stricti, angulis membranaceis scaberrimis. Fol. lata. Fl. 5,6 . In solo humido ad margines aqua. rum et paltudum frequens. 
9. C. muricata: spica androggyna composita, spicul. ovat. superne mascul. approximatis, stigm. 2, fructib. horizontalib. ovat. plano-convex. bidentatis, basi marginatis, margine ciliato-serratis. L. V. IV. 231.

A. 1-2' 24. Rad. caespitosa. FI. 5, 6. Fructus glumas ovatas acuminatas aequantes. In humidis graminosis, in sylvis, ad fossas etc. frequens. 'Teg. Papenb. Grunw. etc.

10. C. stellulata (Gooden): spica androgyna composita, spicul. subquatern. remotiuscul. inferne mascul, stigm. 2, fructib. ovato-acuminat. 2-dentatis margine ciliato-serrat. horizontalibus, glum. ovat. acut. L. W. IV. 236.

A. $\frac{r}{4}$ 24. Fl. 5, 6. Habitus antecedentis. In solo liumido, in pratis, sylvis etc. Grunw. Jungfh. Teg. Cöpn. Heide.

11. C. remota: spica androgyna composita, spicul. altcrn. renot. inferne mascul., bract. foliaceis longissim. suffultis, fructib. ovat. acuminat. bifid. compressiusculis, L. W. IV. 239.

A. $\frac{3}{4}-1 \cdot 24$. Rad. caespitosa. Fl. 5, 6. Glumae et spiculae virentes. In lncis umbrosis humidis sylvarum. Teg. Papenb. etc.

12. C. elongata: spica androgyna composita, spicul. subnovenis approximat. inferne mascul. altern. oblongis, stigm, 2, fructib. oblong. attenuat. 
nervos. patulis gluma ovata obtusa longioribus. L. W. IV. 240.

A. 1-"24. Rad. fibrosa caespitosa. Fol. scabra. FI. 5, 6. Bracteae breves. Glumae fuscae. In locis humidis frequens. Thiergarten. Grunw. etc.

13. C. curta (Willdenow): spica androgyna composita, spicul. subsen. altern. approximat. cylindricis inferne mascul., stigm. 2, fructib. ovat. plano-convex. acutiusculis ore integris erect. gluma ovata acuta longioribus. L. IV. IV. 24 t.

A. $\frac{\pi}{2}-1 \frac{\pi}{2} \cdot 2$. Rad. fibrosa, caespitosa. Fl. 5, 6. Glumae et fructus ex pallide fusco viridescentes. In humidis graminosis haud rara. c) Spicis androgynis compositis paniculatis.

14. C. paradoxa (Willdenow): spic. androgynis anguste paniculat. superne mascul., ram. inferiorib. remotiusculis, stigm. 2, fructib. subrotundo. ovat. rostellat. 2-dentatis, margine superne ciliato. serratis. L. W. IV. 243.

A. 2-3'24. Fl. 5, 6. Glumae ovatae fuscescentes margine lato albido. In solo humido hinc inde. Jungfh. Grunw, etc.

15. C. paniculata: spic. androgyn. paniculat. superne masculis, stigm. 2 , fructib. ovat. superne marginat. bidentatis, margine ciliato-serratis, culmo triquetro $\nabla$. subtereti. 
Carex teretiuscula Gooden. S. I. 468.

$\alpha$. vilgaris. $\beta$. teretiuscula. A. I-3' $4 . \mathbf{F}$.

5 , 6. In paludibus et prope aquas frequens.

d) Spica androgyna terminali, reliquis distinctis foemineis.

16. C. Buxbaumii (Thahlenberg): spica androgyna pedunculata obovata inferne mascula, 우 subternis remotis subpedunculatis, stigm. 3 , fructib. ellipticis triquetr. obtus. obsolete bidentat. glumam oblongam mucronatam subaequantibas. L. W. IV. 252.

A. $1 \frac{r}{2}-2 \frac{r}{2}=4$. Fl. 5, 6. Gluma atro-fuscac. Fructus lutescentes. In pratis paludosis rarius. Zwisch. Fasanerie u. Willmersd. Otto. S.I.469.

e) Spica $\sigma^{\top}$ terminali solitaria, reliquis distinctis $\$$, culmo vaginato aphyllo.

17. C. digitata: spica $\sigma^{\pi}$ solitaría sessili, $q$ sub. ternis linearib. remotiuseulis, stigm. 3 , fructibu nbovat. rostrat. triquetr. pubescentibus squamam obovatam mucronatam aequantibus, bracteis vaginat. obliquis mucronat. margine membranaceis. L. W. IV. 256 .

A. $\frac{\pi}{4}-\frac{3}{4} \cdot 2$. Fol. plana lata. Fl. 4, 5. Glumae fuscae. Fructas pubsscentes. In sylvis frondosis. Papenb. W. Schönh.

f) Spica mascula terminali subsolitaria, re* liquis distinctis o, culmis foliosis. 


\section{- Fructibus pubescentibus.}

18. C. pilulifera: spica $\sigma^{\top}$ solitaria, $q$ subternis approximat. elliptico-subrotund. sessilibus, stigmat. 3, fructib, subrotund. breve rostrat. pubescentib. squama oblonga acuta brevioribus, culmo decumbente. L. W. IV. 259.

A. $\frac{1}{2}-1 \frac{T}{2} \cdot 24$. Fl. 5. Spicae ㅇ 2-4. Glumae 오 fuscae nerso medio viridi, margine albido. In sylvis sublumidis et in campis circum paludes. Jungfh. Grunw. Cöpn, Heide etc.

19. C. collina (Willdenow): spica $\sigma^{\top}$ solitaria, 오 subgeminis approximat. ellipticis sessilibus, stigm. 3 , fructib. oblong. brevissime rostrat. pubescentibus, squamam obovatam subaequantibus, fol. radicalib. annotinis culmo longioribus. L. IV.IV. 26o.

F1. 5. 24. In graminosis siccis sylvarum. Spand. Kth. Falkenhagen. S. Grunw.

20. C. ciliata (Schkuhr): spicula ơ solitaria', 오 subgeminis approximat. oblong. sessilibus, stigm. 3, fructib. subrotundo-obovat. pubescentibus, gluma oblonga obtusa majoribus. L. IV. IV. 261.

Fl. 5. 24. Fol. glauco-viridia. Spica $\sigma^{\top}$ clavata. Glumae fuscae margine albido culiato. In graminosis sylvarum frequens.

21. C. praecox (facquin): spica mascula solitaria, $q$ geminis approximatis oblongis sessilibus; stigmat. 3, fructib. subgloboso-triquetr. pubesceu- 
tibus glumam ovatam acutam aequantibus. L. W. IV. 262 .

Fl.5. 24. Fol. ex lutescenti viridia. Fl. 4. Glumae fuscae. In solo sicco sylvarum et camporum. Grunw. Jungfh. etc.

22. C. tomentosa: spica mascula solitaria, 옹 ger minat. cylindraceis subapproximat. sessilibus, stigm. 3, fructib. subrotund, tomentos, gluma orata acuminata majoribus. L. W. IV. 263.

A. $1 \frac{\mathrm{T}}{2}-2^{\circ} 24$. Fl. 5. Spicae 오 plerumque 2 . In pratis rarissime. Charlottb. Kth.

23. Co recurva (Hudson): spic. mascula geminata, \& cylindricis subternat. pedunculat. pendalis, stigm. 3, fructib. elliptic. obtus. scabriuscul. glumam ovato-cuspidatam aequantibus. L IV. IV. 298. A. $\frac{3}{4}-1^{\prime} 4$. Rad. repens. Fol. et culmus glaucescunt. Fl. 5. Fruct. ferrugineo-viridis. In solo humido camporum et sylvarum. Thierg. Jungfh.

24. C. filiformis: spicis mascul. geminis, +9 geminis ovato-oblong. sessilib. distantibus, stigm. 3 , fructib. elliptic, bifurcat. lanatis glumam oblongogo-lanceolatam subaristatam aequantibus. L. W. IV. 303 .

A. $1 \frac{\pi}{2}-3^{\prime} 4$. Riad. repens. Fol. convoluta scabra. Fl. 5. Spicae o $1-2$. Glumae fuscae. Fructus lanugine subferruginea tecti. In pa- 
Iudibus et in limosis frequens. Temph. Kth. Grunw. Jungfh.

25. C. hirta: spic. mascul. binis, \& tribus ob. Iong. remot. inferiorib. breve pedunculatis, stigm. 3 , fructib. oblong. acuminat. hirt. bicuspidat. gluma oblonga aristata majoribus, vagin. foliisque subhirtis. L. W. IV.311. H. A. V. 9.

a. terrestris. F. aquatica. A. $\frac{1}{2}-1 / 2$. Rad. repens. Habilus C. arenariae. Planta variabilis in solo sicco et humido obvia.

* Fructibus glabris:

26. C. supina (Willdenow): spica mascul. solitaria, 오 subsolitaria subrotunda sessili approximata, stigm. 3, fructib. elliptico-triquetr. rostrat. ore emarginat. glumam ovatam subaequantibus. L. W. IV. 265 .

A $\frac{x}{4}-\frac{1}{2} \cdot 4$. Fl. 5. Spicae 오 1-3. Habitus C. piluliferae. In collibus graminosis. Pichelswerder. Ruthe. S. I. 475.

27. C. limosa: spicula mascula solitaria, 9 binis ovat. pedunculat. pendulis, stigm. 3, fructib. ellipt. compresso-triquetris brevissime rostellatis, ore integro, glumam mucronatam aequantibus, bracteis amplexicaulibus. L. W. IV. 293.

A. $\frac{1}{2}-1 \frac{\pi}{2} \cdot 24$. Rad. repens. Fl. 5. Culmi glaucescunt. Glumae ex fusco et viridi pictae. Fructus albido-virentes. In paludibus turfosis hinc inde frequens. Grunw. Jungfh, etc. 
28. C. pallescens: spica masc. solitaria, nis pedunculat. cernuis ellipticis, stigm. 3 , fructib. obovato-oblong. obtus, glumam oblongam cuspidato-mucronatam aequantibus, fol. pubescentibus, L. W. IV. 291.

A. $\frac{3}{4}-I \cdot 4$. Rad. caespitosa. Culmi foliosi. Fl. 5. Glumae et fructus virides v. fuscescen. tes. In sylvis humidis et in pratis apertis. $\mathrm{Pa}$. penb. Jungfh. Thierg. etc.

29. C. Pseudo-Cyperus: spica masc. solitaria, 오 quaternis geminat. pedunculat. pendul. cylindricis, stigm. 3, fructib. ovato-lanceolat. bicuspidat. rellexis glumam setaceam aequantibus. L. Wr. IV. 295.

A. 2-3' 4. Rad. caespitosa. Fol. pallide viridia. FI. 5. Glumae et fructus virides. Ad margines aquarum frequens.

30. C. Drymeja: spica masc. solitaria, 우 quaternis remot. filiformib. pedunculat. cernuis, stigmatib. 3, fructib. ovat. rostrat. bifidis glumam ovatam membranaceam mucronatam subaequantibus. L. W. IV. 296.

A. 1-2' 24. Rad. fibrosa stolonifera. Fol. lata non rigida. Culmi foliosi. Fl. 5, 6. spicae foemin. $3-6$. Fructus virides. In sylvis humidis umbrosis. Teg. Papenb. W.

31. C. flava: spica masc. solitaria, 오 subternis subapproximatis ellipticis incluse pedunculatis, 
stigm. 3, fructib. ovat, reflex. rostratis, ore curvato bidentato, gluma ovato-lanceolata longioribus. L. W. IV. 268 .

a. elatior. 3. Oederi. A. $\frac{1}{4}-1$ ' 24. Herba lutescenti-viridis. Spicae ㅇ $1-4$. In pratis turfosis $\alpha$; in paludosis $\beta$.

32. C. distans: spica masc. solitaria, $ᄋ$ tribus ovatis distantibus, suprema sessili, reliquis pedunculatis, stigm. 3, fructib. ovat. acuminat, bicuspidat. glumam ovatam acuminatam subaequantibus. $I$. IV. IV. 271.

A. 1-1 $\frac{1}{3}$. Fl. 5, 6. Spicae 우 2-6. Fructus virides. Glumae fuscescentes. In pratis humidis sylvarum et apertis. Jungfh. Grunw. etc.

33. C. panicea: spica mascul. solitaria, 옹 subbinis remotiusculis, suprema subincluse pedunculata, stigm. 3, fructib. ovat. obtus. squama ovata majoribus. L. IV. IV. 280.

A. $\frac{1}{2}-1 \cdot 2$. Fl. 5. Spicae 9 I-3. Glumae nigro-fuscae. Fructus lutescenti-virentes. In pratis humidis frequens.

34. C. cespitosa: spica masc. solitaria, 우 cylindraceis obtus, bin. $\nabla$. ternis distantibus, infima brevissime pedunculata, stigm. 2 , fruct. ovat. obtus. v. acutiuscul. ore pertusis, gluma oblonga v. lanceolata obtusa $\nabla$. acutiuscula majoribus.

C. stricta. L. W. IV. 287 .

$$
\text { F. B. }
$$


a. terrestris. B. palustris. A. 2"-2, 24. Fl. 5 . In solo humido et paludoso frequentissima. C. strictam L.W.1.1. esse varietatem Caricis cespitosae, minime vero, ut S. I. 479 visum, C. acutae, persuasum habemus; solet enim $C$. cespitosa in solo humido non inundato eam, quam C. cespitosam vocarunt, formam, in undatis autem quam $C$. strictam appellarunt, induere.

35. C. acuta (Willdenow): spic. masc, binis ternisve, \& subquaternis subpedunculat. subnutantib. cylindraceis remotis, stigm. 2 , fructib. oblong. brevis. sime rostellatis, ore integro pertuso glumam oblongam acutam subaequantibns. L. W. IV. $304 \cdot$

A. $1-2^{\prime} 24$. Fl. 5,6. Herba glaucescens. Fol. scabra. Planta admodum variabilis. In solo lumido et paludoso undique.

37. C. paludosa (Gooden.): spic. masc. binis, ternis erect. cylindraceis attenuatis, inferiorib. pedunculatis, stigm. 3 , fructib. ovat. nervos. bidentat. gluma lanceolata aristata latioribus eamque aequantibus. L. W. IV. 305.

A. 2-3' 24. Fl. 5, 6. Medium tenet inter C. acutam et $C$. ripariam. In aquis et paludibus hinc inde. Thierg. Rummelsb. Jungfh. ctc. 38. C. riparia (Gooden): spic. masc. tribus, 우 tribus erect. cylindraceis attenuatis, inferiorib. pedunculatis, stigm, 3, fructib. ovat. rostrat. multi- 
nerv. bifurcat. latitudine et longitudine glumae ovatae aristatae. L. WV. IV. 306.

A. $2-4^{\prime} 4 \mathrm{Fl} 5,6$. In aquis, fossis, rivis, fuminibus. Junglh. Thierg. Spand. etc.

39. C. vesicaria: spicis masc. tribus, 우 subgeminis pedunculat cylindraceis, stigm. 3, fructib. oblong. inflat. rnstrat. bicuspidat. gluma lanceolata majoribus, culmo acute triquetro. L. W. IV.307. A. $1-2^{\prime} 4$. Rad. repens. Fol pallide vi. ridia. F1. 5, 6. Glumae fuscae nervo viridi Spicae $1 \div 4$. In locis paludosis frequens. Jungfh. Thierg. Grunw. etc.

40. C. ampullacea (Goodenough) : spic. masc, tribus, 우 binis cylindraceis breve pedunculat, erectis, stigm. 3 , fructib. subglobos. inflat. rostrat. bifurcat. gluma lanceolata majoribus, culmo obtuse trigono. L. W. IV. 308.

Habitus praecedentis. 24. Fol. glauca. F1.5,6. In solo humido, pratis, ad aquas, paludes. Thierg. Jungfh. Grunw. etc.

\section{ORDO III. TETRANDRIA.}

.373 IITTOREIIA.

Flor. radicales. Flor. $\sigma^{\top}$ pedunculati, Cal. 4-partitus. Cor. 4-fida. Stam. longissima. Flor. siles. Cal. squamae angustae. Cor. tubulosa 
apice 3-fida. Achacn. corolla persistente inclusum.

1. L. lacustris. L. IV. IV. 330 .

Rad. stolonifera. 4. Fl. 6, 7. Fol. linearia basi dilatata vaginantia flores 9 amplectuntur. Flor. $\sigma^{7}$ pedunculati. In littoribus lacuum in. undatis satis frequens. Temph. S. Weifss.

$$
\text { 37千. AIXUs. }
$$

Flor. $\sigma^{7}$. Amentum cylindricum, squamis cuneatis, apice incrassatis truncatis 3 . floris Invols cri squama subrotunda. Cal. 4-partitus. Cor.o. Flor. ơ. Amenta ovata squam. 2-floris. Cal.o. Cor. o. Nux compressa inalata 1 -locularis I. sperma.

1. A. glutinosa (Willdenow): fol. glutinosis ad axillas venarum subtus villosis v. cuneato-subro. tund. emarginatis, $\nabla$. oblong. pinnatifid. $v$. sinuatis. L. IV. IV. 334 .

Betula alnus $L$.

a. emarginata. B. quercifolia. A. 10-70' h. Fl. 2-3. Arbor notissima in locis humidis et paludosis undique obvia.

2. A. incana: fol. rotundato-ovalibus et ovaliovatis acut. subtus pubescentibus in venarum axill. glabris, stipul. lanceolatis H. F. 152.

Antecedenti similis arbor hinc inde culta. Thierg. 
375. NonU s.

Flor. ${ }^{7}$. Amentum nudum. Cal. 4-partitus. Cor.o. Flor. ㅇ. Amenta nuda. Cal.4-5-sepalus. Cor.o. Styl. 2. Achaen. a calyce in baccam mutato coalito tectum. Omnes calyces amenti baccae compositae similes.

1. MI. alba: fol. ovat. basi subdimidiato-cordat. integr. lobatisve inaequaliter serrat. glabr, sublaevibus. L. IV. IV. $36 \mathrm{~s}$.

A. $20-30^{\prime}$ 苂. Fl. 5, 6. Frnctus albi v. rubicundi. Arbor Orientalis hinc inde culta. Off. 3;6. UnticA.

Flor. o. Cal. 4-sepalus. Cor, o. Nect, centrale cyathiforme. Flor. 우. Cal.2-4-sepalus. Cor. 0. Caryopsis compressa calyce persistente cincta.

1. U. urens: fol. oppositis ellipticis sub-5-nerviis argute serratis, spicis glomerat. geminatis. A. $\frac{T}{3}-1 \frac{\pi}{2}{ }^{\prime} \odot$. F1. $6-10$. Tota planta pilis urentibus tecta. In ruderatis, agris etc. frequentissima.

2. U. dioica: fol. opposit. cordat. ovato-lanceolat. grosse serratis, flor. dioicis, spicis paniculat. glomerat, geminat, petiolo longioribus. L. W. IV. 352.

A. 1-4'4. Rad, repens. FI. 6-10. Pili urentes. In agris ad vias, ruderata etc, vulgaris, 


\section{ORDO IV. PENTANDRIA. \\ 377. XAN THIUM.}

Flor. $\sigma^{\nearrow}$. Involucr. imbricatum multiflorum, Cor. infundibuliform. 5-fida. Receptac. paleaceum. Antherae 5. Flor. ㅇ․ Involucr. 2-phyllum biflorum. Cor. 0. Achaen. 2 in involucro 2-loculari persistente birostri.

1. $X$. strumarium: caule inermi, fol, cordat. 3-ner. vatis. L. IV. IV. 373.

A. $1 \frac{1}{2}-2^{\prime} \odot$. Fl. 8, 9. In ruderatis, ad pa= gos, sepes etc.

379. A MI RA NTHU S.

Floris ơ et ㅇ․ Cal. 3-5-phyllus. Stam. 3--5. Caps. 1-locular. circumscissa calyce cincta I - sperma.

1. A. Blitum: caule diffuso, fol. ovat. retusis, glomerul. subspicatis, Ror. 3-pliyl!. $\sigma^{\top}$ triandris. L. W. IV. 387 .

A. $\frac{1}{2}-1^{\prime} \odot$. Fl. 7,8 . In ruderatis, plateis etc. fiequens.

\section{ORDO V. POLYANDRIA.}

379. CERATOPH YLIUM.

Flor. ơ. Cal. simplex multipartitus. Antherae sub 4.loculares, Flor. ㅇ. Cal, simplex multi- 
partitus. Cor. o. Pistillum 1 stylo filiformi. Achaen. 1 nucamentaceum.

1. C. demersum: fol. dichotomo-bigeminis, fruclib. trispinosis. L. W. IV. 405.

Planta tota submersa. Fol. setacea verticillata. $4 \mathrm{Fl}$. 6 . In aquis stagnantibus, fossis, lacunis etc. frequens, Grunw. Jungfh, etc.

2. C. submersum: fol dichotomo-trigeninis, fructib. muticis. L. WT. IV. 405.

Antecedenti affinis 2. Fl.6. In fossis, b. d. Inralidenhause W.

\section{MI Y IOPHYIIUM.}

Floris $\gamma^{7}$ et petal. caducis. Stam. 8. Sigm. 2-4 sessilia. Achaen. 2-4 infera nucamentacea.

1. M. spicatum: fol omnib. pinnat. capillaceis, spica terminali verticillata nuda. L. W. IV. 406.

Planta immersa. 24. Caul. subdichotomi. Fol. quaterna verticillata. Fl. 6,7 . In aquis stagnantibus. Grunw. Jungfh. etc.

2. M. verticillatum: fol. pinnat. capillaceis, superiorib. pectinato-pinnatifidis, Hor. axillarib. verticillatis. L. W. IV. 407.

Habitus $M$. spicati. 4 . Fol. quinata. Tota planta! laetius viridis. In locis antecedentis trequens. 


\section{SA GITTARIA.}

Floris $\sigma^{7}$ et . . Cal. 3-sepalus. Cor. 3-petala. Stam. numerosa. Achaen. membranacea numerosa.

1. S. sagittifolia: fol. lanceolat. acuminat, sagit tátis, lob. lanceolat. rectis, scapo simplici. L. W. IV, 408 .

a. latifolia. $\beta$. angustifolia. Rad. crassa 4 . Fl. 6, 7. Cor. alba. Achaenia in capitulum disposita. In aquis, fossis, paludibus etc. frequens.

382. POTERIUM.

Flor. $0^{7}$. Cal. 4-sepalus, sepal, receptaculi collo insertis deciduis. Cor. o. Stam, 20 v. plura germinis collo inserta. Flor. ‥ Cal. et Cor. maris. Germina 2 receptaculo inserta. Achaen. 2 receptaculo inclusa.

1. P. Sanguisorba: inerme pubescens $\nabla$. glabrum, caulib. subangulatis. H. A. VIII. 23.

A. $\frac{3}{4}-1$ ' 4. Rad. sublignosa. Fol. pinnata, foliol. subpetiolat. subrotund. obtuse serratis. Fl. 6, 7. Flor. capitato-spicati. In collibus calcareis siccis. Kalkb.

\section{BETULA.}

Flor. $\sigma^{\top}$. Amentum cylindricum imbricatum, squam. 1-floris. Cal. squama 3- - 5-partita Cor. o. Stam, 6-12. Flor. ㅇ. Amentum subcy* 
lindricum imbricatum, squamis $2 \cdot-3$-floris. Cal. o. Cor. o. Styli 2. Samara 1-sperma.

1. B. alba: fol. deltoideis acuminat. Uuplicatoserrat. glabris, petiol. glabr. pedunculo longioribus, strobilorum squam. lob. lateralib rotundatis. L. IV. IV. 452.

a. vulgaris $\beta$. pendula. $\%$ macrocarpa. A. $60-80$. 7. Fl. 4,5 . In sylvis humidis et sub. humidis arenosis frequens.

2. B. pubescens (Ehrhardt): fol. subdeltoideo. subcordat. acuminat. duplicato-serratis utrinque praesertim subtus et in ramul. pubescentibus, juniorib. velutinis, petiol. pubescentibus, strobilorum squam. lobis lateralib. rotundatis. II. F. 166. A. $20-30^{\prime}$ \%. Fl. 4, 5. Strobilorum squamae ciliatae. Samarae pubescentes. In paladibus et turfosis locis lincinde. Grunw. Talsd. Steglitz etc.

\section{S4. QUERCU3.}

Flor. $\sigma^{\top}$. Amenta nuda. Cal. 5--9-fidus. Cor.o. Stam. 5-10. Flor. ㅇ. Cal. 1.sepalus, extus im. bricato-squamosus. Cor. o. Stigm. 2-5. Nux coriacea basi calyce coriaceo-lignescenti cincta I-sperma.

1. Q. Robur: fol. oblong. sintrat. basi rotundat. v. subcordato-cuneatis, lobis rotundatis, juniorib. subtus pubescentibus, adultiorib. glabris, fructib. 
sessilibue, cal. fere semiglobos. verrucoso-squamos. glabris, nuce ventricoso-oblonga. H. F. 159 .

A. $20-120^{\prime}$ 万. Fl. 5. Flor. cum foliis apparent. In sylvis frequens. Off

2. Q. pedunculata: fol. oblong. profunde sinuatis, basi reflexo-cordatis, lob. rotundatis, juniorib. senioribusque glabris, fructib. pedunculatis, cal. fructus fere semiglobosis verrucoso-squamat. glabris, nuce cylindrico-oblonga. II. F. I6c.

Affinis praecedenti $\hbar$. Floret prius. Truncus crassior et altior. In locis iisdem. Off.

$$
\text { 385. conyuus. }
$$

Flor. ऽ. Amentum cylindricum imbricatum, squam. x-floris. Cal. squama 2-partita basi cum amenti squama counata. Cor. o. Stam. S. Flor. ㅇ․ Cal. 2-partitus lacerus floriferus vix conspicuus. Cor. o. Słyl. 2. Nux calyce cincta 1-locul.1-sperm. 1. C. Avellana: fol. rotundat. subcordat. acuminatis, stipul. oblong. obtusis, calyce fructus campanulato apice patulo lacero.dentato. L.W.IV.470.

A. $10-20^{\prime}$ 7. Fl. $2-3$. In sylvis humidius. culis frequens.

386. FACUs.

Flor. $\sigma^{7}$. Amentum nudum. Cal. 1.sepal. campanulat. 5-fidus. Cor. 0. Stam. 8-12. Flor. . Involucr, 4-fidum setaceum 2.florum, Cal, su. 
perus 6-dentatus. Cor. o. Nices 2 involucro coriaceo aculeato 4-fido inclusae.

1. F. sylvatica: fol. oblique ovato-ovalibus, acut. inaequaliter dentat. glabr. margine ciliatis. II. .F. 164 .

A. $50-60^{\circ}$ 7. Fl. 5. In sylvis frequens. Grunw. Thierg. etc.

$$
\text { 387. carpinus. }
$$

Flur. $\sigma^{7}$. Amentum cylindricum imbricatum, squam. 1-floris. Cal.o. Cor, o. Stam. 10-20. Flor: ㅇ․ Amentum gracile imbricatum, squam. 2-floris. Involucr. squama ciliata decidua. Cal. sup. 4--6-dentat. persistens. Styl. 2. Nux ovata compressa 1-sperma.

1. C. Betulus: fol ovali-oblong. acuminat. duplicato-serrat. approximate costato-venosis, strobili squamis 3-partitis, laciniis serrat. medio elongato lanceolato-lineari. H, F. I68.

A. $30-40^{\prime}$ 方. Fl. 5. In sylvis frondosis frequens.

\section{PLATANUS.}

Flor. $\checkmark$. Amenta globosa, squam. 1-floris. Cal.o. Cor. o. Antherae duplicatae staminibus latera. liter adnatae. Flor. Q. Amenta globosa, squam. 1-floris. Cal. o. Cor. o. Styl. 1 stigmate reflexo. Achaen. stylo mucronatum basi pilis cinctum. 
1. P. acerifolia (Willdenow): fol. cordat, 5-lob. remote dentat. basi truncatis, juniorib. subtus pubescentibus, stipulis dentatis. H. F. 171.

A. 50-60' 万. Arbor orientalis. Fl. 5. Flor. cim foliis apparent. Cortex quotannis deciduus. Frequens ad vias.

\section{ORDO VI. MIONADELPHIA.}

\section{PINUS.}

Flor. ơ. Cal. squamosus. Cor. o. Stam. plurima racemosa. Antherce duplicatae cum squama connatac. Flor. ㅇ. Amentum inibricatun, squam. 2.floris. Involucr. squama, Cal. o, Cor. o. Styl.1. Nux plerumque alata.

$$
\text { * Foliis fasciculatis (Larix). }
$$

1. P. Larix: fol. fasciculat. deciduis, strobil. ovato-oblongis, squamis margine relex. laceris, bracteol. panduriformibus. L. W. IV. 362 .

A. $60-80^{\circ}$ 万. F1. 5. In sylvis hinc inde culta. Thiery. Hasenh. Jungfh. etc.

* Foliis quinis.

2. P. Strobus: ramul remot, strbcicatrisat. glabris, fol. quinis, strobil. cylindraccis apice attenuat. lax. foliis longioribus. L. WV. IV. 50 r.

A. $60-80^{\prime}$ h. Fl. 4-5. Cortex trunci lae. vis. Arbor frequentissime culta. Thiergart. Jungfb, etc. 
* * Foliis geminis (Pinus).

3. P. syluestris: fol. geminat. rigidis, strobil. sub. gemin. orato-conicis, basi rotundat. longitudine foliorum. L. W. IY. 49\%.

๘. vulgaris. B. rubra. A. so-120' t. Fl. $5-5$. Arbor totas syluas constiunens.

**** Foliis solitariis planis distiche inversis (Picea).

4. P. Picea; fol. solitariis plan. integr. emarginat. distiche inversis, strobili squam. obtusissimis adpressis. L. W. IV. 504 .

A. $150-160^{\prime}$ 古. Fl.5. Arbor hinc inde culta. Thierg. etc.

***** Foliis solitariis tetragonis subsecundis (Abies).

5. P. Abies: ramul. glabris, fol. solitar. tetragogonis, strobil. cylindraceis, squam, xhombeis complanat. margine repand, erosis. L. W. IV. 506.

A. 160-190: Fl. 5, 6. Culta in sylvis hinc inde Thierg. Jungfh. etc.

390. THU J A.

Flor. Ђొ. Amentum subrotundum imbricarum squam. I.floris. Cal. o. Cor. o. Antherae plerumque 4 subsessiles. Flor. ㅇ. Amentum oblongum imbricatum, squam. 2-floris. Cal. o. Cor. o. Styl, 1. Nux 2-alata v. nuda plerumque 1 sub squama coriacea concara. 
1. T. occidentalis: ramis folios. compresso-ancipitibus, fol. quadrifariam imbricat. adpress. ovatoyhombeis medio tuberculatis, strobil. obovato-ovalibus, squam. adpressis, interiorib. truncat, infra apicem gibbosis. H. F. 177 .

A. $20-60^{\prime}$ 古. Fl. 5. Colitur hinc inde. Thierg. etc.

\section{1, B R Y O I A.}

Flor. ${ }^{\nearrow}$. Cal. 5-dentat. superus. Cor. 5-partita. Filam. 3. Flor. 오. Cal. et Cor maris. Styl. 3-fidus. Bacca subglobosa polysperma infera.

1. B. alba: fol. cordat. 5-lob. dentat. calloso-asperis acutis, flor. corymbos. monoicis, cal. 오 corollam aequantibus. H. A. VI. 23.

Rat? napiformis tuberculosa. Caul. scandentes. 2. Fl. 6, 7. Cor, flavo-viridicans. Filam. glabra. Bacca nigra. Ad sepes et in dumetis hinc inde. Willmersd S. Spand, Off.

2. B. dioica (facquin): fol. cordat. 5-lob. dentat. calloso-asperis acuminatis, flor. corymbos. dioicis, cal. 오 corolla brevioribus. H. A. VI. 24 .

Habitus B. albae Rad. non tuberculosa. 24. Fl. 5. Flor. majores. Filamenta pilosa. Bacca scarlatina. Similibus locis. Panckow etc. 


\section{I. X XI. D I O E C I A. ORDO I. MONANDRIA.}

392. N A J A s.

Flor. ช. Cal. o. Cor. o. Anthera sessilis 4-locul. 4-valvis. Flor. ․ Cal. o. Cor. o. Ovarium stylo 2-5-fido. Achaen. drupaceum mucronatum.

1. N. major (Roth): fol. lanceolat. dentat. plan. apice tridentatis. Roth germ. II. 99 .

Najas monosperma et tetrasperma. Kth. 255.

A. 3-5" ○. Caul. dichotomus inferme radicans. Fol. opposita sessilia sinuata dentata. Fl. 7 , 8. In fuucio arenoso lacuum. Schlachtensee. S. Teg. Kalksee.

2. N. minor (Allioni): fol. lineari-subulat. recurvat. aculeato-dentatis. Roth germ. II. 500. S. I. 499 .

Caulinia fragilis. L. W. IV. 182. Kth.23g.

Praecedente tenerior et fragilior. Fol. cauJina inferiora remotiora, superiora conferta

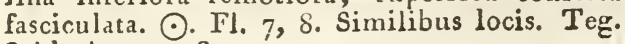
Schlachtensee S.

\section{ORDO II. DIANDRIA.}

393. SA IIX.

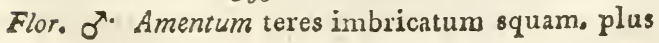


minusve ciliat. 1-floris. Cal. o. Cor. o. Nect. glandula staminifera in basi squamae. Flor. ㅇ․ Amentum, cal. et cor. maris. Nect. glandula germinifera in squamae basi. Styl. bifidus. Caps. 1locul. 2-valv. Sem. plurima basi desmate cincta. * Foliis glabris serratis.

1. S. triandra: fol. lanceolat. acuminat. basi obtus. serrat. subtus glaucescentibus, ament. coaetaneis triandris, germinib. pedicellat, oblong. :uber. culat. glabris, stigm. sessilibus. L. WV. IV. 65\%.

A. $8-14^{\prime}$ 万. Fl. 5. In solo lumido et paludoso. Thierg. Charlottb. etc.

2. S. Russeliana (Smith): fol. lanceolat. acuminat. serrat. glabris, ament. coaetaneis triandris, germinib, pedicellat. subulat. Laevibus, styl. elongatis. L. W. IV. 656.

Fl. 4-5 万. Rami flexiles. Ad pagos, vias, fossas passim. Schöneb. S. Teg. etc.

3. S. pentandra: fol. olliptic. acuminat. serrat. glabris, petiol. superne glandulosis, amentis serotin. 5-andris, germinib. lanceolat. glabris. L. W. IV. 658 .

A. $20-40^{\prime}$ t. Fl. 5. In solo paludoso hu. mido frequentissima. Off.

4. S. Meycrina (Rostkovius): fol. oblongo-lanceolat. acuminat. glabris, petiol. superne glandulosis, 
ament. serotin. triandris, stam. reflexis, germinib. lanceolat. glabris. W. Baumz. 427 .

A. $20-30^{\circ}$ 古. Fl. 5. Ad vias et fossas. Pul. vermühlen.

5. S. vitellina: fol. lanceolat. acuminat. glabris serratis subtus glaucescentib., petiol. pilosiusculis, ament. coataneis, germinibus sessilibus lanceolat. elongat. glabris, stigm, sessilib. bilobis, ramis vi. tellinis splendentibus. H. F. 183.

A. $40-60^{\circ}$. . In solo humido frequens:

6. S. fragilis: fol. lanceolat, acuminat. concolo. rib. glabris glanduloso-serratis, ament. coaetaneis, nectario masculorum gemino, germinib. subsessilib. elongat. oblong. glabris. II. F. 183.

A. $20-45^{\prime}$ t. Rami fusci fragiles. In locis humidis arenosis vulgaris.

7. S. Helix: monandra erecta, fol. lanceol. acuminat. serrulat. glabris, stylo elongato filiformi, stigm. linearibus. L. W. IV. C 72.

A. 10-12, h. Rami cincrei v. fuscescenti. rubri splendentes. Fl. 4, ante fol. eruptionem. Ad margines aquarum, Panck, $u$, Schönh. W. Spand. W.

$$
\text { * * Foliis villosis. }
$$

8. S. repens: fol, ovali.lanceolat, recurvato-acut, supra pubescentibus, subtus subsericeis, caps. priF. B. 
mum sericeo-pubescentibus demum sabglabris, ramis depress. exstipulatis. H. F. 188.

A. $1-2 \cdot$ t. Fl. $4-5$ ante foliozum eraptionem. In solo arenoso humido frequens.

9. S. fusca: fol. ovali-lanceolat. utrinque attenuat. subintegerrim. acutis, supra glabris, subtus serice-tomentosis, stipul. lanceolato-subulatis, capsulis lanceolat. pedicellat. sericeis. H. F. 188 .

A. $3-4^{\prime}$ t. Fl. ante foliorum eruptionem 4-5. In solo humido et sicce Panck. Schönhaus. etc.

10. S. incubaces: fol. lanceolat, utrinque attenuat. integr. convexis supra glabris, subtus incan. sub. -riceis, germinib. elongat. ovato-oblong. sericeis pedicellatis, stigm. subsessilibus. L. IV. IV. 696 .

A. $2-3^{\circ}$ 南. Fl. $4-5$ ante foliorum eruptionem. In pascuis humidis arenosis hinc inde.

11. S. rosmarinifolia: fol. lineari-lanceolat. sub. integerrim. plan. supra pubescentibus, subtus sericeis, germinib. elongato-oblong. villos. pedicellatis, stylo elongato. H. F. $18 \mathrm{~g}$.

A. $1-1 \frac{\pi}{2} / \hbar$. Fl, 5. In solo paludoso frequentissima.

13. S. uliginosa (IVilldenow): fol. obovat. breviter recurvato acuminatis apicem versus undulato-deneatis, supra viridib. pubescentibus, subtus rugosoveros et incano-rillosis, atipul, subdimidiato-cor- 
dat. dentatis, caps. elongato-oblong. pedicellatis, stigm. sessilibus. H. F. 191.

A. $4-5$ 古. Fl. $3-4$ ante foliorum eru. ptionem. In locis humidis haud rara.

14. S. aurita: fol. obovat. basi attenuat. breviter acuminat. mucronatis, mucrone recurvato, subserrat., supra viridib. pubescentibus, subtus rugosovenos. griseo-pubescentibus, stipul. subdimidiatocordat. subintegerrimis, caps, elongato.oblong. pedicellatis, stigm. sessilib. H. F. 190.

A. $2-3^{\prime}$ f. Fl. $3-4$ ante fol, evolutionem.

In locis humidis umbrosis.

15. S. cladostemma (Hayne): fol. strbrotundo-obovat. basi subcordat. breviter acuminat. mucronatis, mucrone recurvato, undulato-dentat. supra viridib, pubescentibus, subtus rugoso-venos, incanopubescentibus, stipul. oblique lunat. integr. undu. latis, ament. 1.andris, anther. ramosis. H. F. 190. A. 5-6, 古. Fl. 4-5 ante foliorum eruptionem. Differt a $S$. aurita amentis 1-andris, basi foliorum, filament. solitariis infra divisionem pilosis. In solo humido ad rivulos rarius. Panckow.

16. S. aquatica (Smith): fol, obovato-elliptic. subserrat. acutis, supra viridib. pubescentibus sub. tus glauc. pilosiusculis, stipul. rotundat, dentat, 
germinib. pedicellat, lanceolat. villosis, stígm. sessilibus, L. W. IV. 701 .

A. $\delta-10^{\prime}$ t. Fl. 3,4 ante folia eroluta. In locis humidis, fruticetis etc. non rara. Thierg. 17. S. Capraea: fol. ovat. acuminat. serrat. un. dulatis subtus tomentosis, stipul. sublunatis, caps. renericosis. L. IV. IV. 703.

A. $20-30$ t. Fl. 4, 5. In solo humido, in nemoribus et fruticetis copinse.

18. S. acuminata (Hoffmann): fol. lanceolato-oblong. acuminat. undulat, denticulat. subtus tomen. tosis, stipul. reniformibus, caps. ovato-subulatis. L. W. IV. 704 .

A. $8-10^{\prime}$ F. Fl. $3-4$, antequam folia ap. parent. In nemorosis humidiusculis passim. Strahl. Kth.

19. S. viminalis: fol. subintegerrim. lineari-lan. ceolat. longissim. subtus albo-sericeis, pilis squamarum amenti \& germine longioribus. L. W. IV. 706 .

A. 12-16, 万- 万. Fl. 4, 5 ante foliorum eruptionem. In locis humidis ad margines aquarum. Thierg. Spand. etc.

20. S. alba: fol, lanceolat, acuminat..serrat. utrinque sericeis, serratur. infim. glandulosis, stigm. bipartitis. L. W. IV. 710.

A. $30-40^{\circ}$ t. Fl. 5 post foliorum eruptionem. Ad rias, sepes etc. vulgatissima. 


\section{ORDO III. TETRANDRIA.}

394. VIsCUM.

Flor. o. Cal. 4-partitus. Cor, o. Filam, 0. Anthsrae calycis laciniis insertae. Flor. ㅇ. Cal. 4phyllus superus. Cor. o. Styl. o. Bacca 1-sperma. 1. V. album: caule dichotomo, fol. opposit, ob. ovato-lanceolat. obtus. trinervibus, flor, terminalib. axillaribusque sessilib. plerumque ternis. I. W. IV. 737. H. A. IV. 24.

In arboribus, praesertím in P. sylvestri et Quercubus crescens et in ipsos ramos radices virides, quae postea quasi cum ligno arboris confluere videntur, agens. Fl. 4, 5. Bacca alba. In sylvis haud rarum. Off.

\section{ORDO IV. PENTANDRIA.}

\section{SPIN A CIA。}

Flor. 万'. Cal.5-partitus, Flor. ‥ Cal.2--4-fidus, Cor. 0. Styli 4. Achaen. 1. calyce indurato in. clusum.

1. S. oleracea: fructibus sessifibus, L. W. IV. 1766 .

a. spinosa $\beta$. inermis. A. $\frac{1}{2}-I \frac{T}{2} \bigodot_{\text {. Fol. sa- }}$ gittata et oblongo-ovata. Fl. 6, 7. Flor. axillares. Olus notissimum in agris cultum. 
3.2 DIOZCIA. PEXTATDKIA, OCTANDRIA.

\section{C A N N A B Y s.}

Flor. ơ. Cal. 5-partitus. Cor. o. Flor. ․ Cal. 1-sepalus integer, lateribus hians. Cor. 0. Styli

2. Achaen. nuciforme calyce inclusum.

1. C. sativa: fol. oppositis digitatis. L. W. IV. 768. H. A. VIII. 35.

A. $1-10^{\circ} \odot$. Fl. 6-8. In fimetis, ruderatis, ad vias etc. $v$. in agris culta, Off.

397. HUMUIUS.

Flor. ð. Cal, 5-sepalus. Cor. 0. Flor. ㅇ․ Cal.1-se. palus oblique patens. Cor. x-petala subglobosa germen arcte cingens. Styli 2. Achaen. corolla persistente tectum.

1. H. Lupulus: L W.IV.76g. H.A. VIII:36.

Rad. repens. Caulis scandens. Fol. cordata $3-5$. loba v. integra. Fl. 7, 8. Flor. $\sigma^{\nearrow}$ paniculati, $q$ capitati. In solo meliore subhumido, in sylyis, ad sepes etc. y. culta. Off.

\section{ORDO V. OCTANDRIA.}

398. POPUIUS.

Flor. $\sigma^{7}$. Amentum laxe imbricatum, squam. laceris 1-floris. Cal. turbinato-cyathiformis obli. quus integer. Cor. 0. Flor. ㅇ․ Amentum laxe imbricatum, squam. laceris 1-floris. Cal. subventricoso. cyathiformis obliquus integer. 
Cor. o. Styl. o. Stigm. plerumque 4-partitum, rarius 3- v. 6-partitum. Caps. 2--3-valvis, 1-locularis. Sem. plurima basi desmate cincta. I. $P$. alba: fol. cordato-subrotund. lobat. dentat. subtus niveo-tomentosis, petiol. subcompressis summorum foliorum dimidium folium aequantibus, amentis ovatis. H. F. 200.

A. $80-100$ 南. Fl. 3-4. Ad vias frequen. tissima. Stadt. Thierg. Teg. etc.

2. $P$. canescens (Smith): fol. subrotund. angulato. repand. deritat. subtus incano-tomentosis, petiol. subcompressis, summorum folior. dimidio folio brevioribus, amentis cylindrac. laxis. H. F. 199.

Antecedenti simillima et vix discernenda. Ad vias hinc inde obvia.

3. P. tremula: fol. subrotund, acut. angulatodentat, utrinque glabris, petiol. valde compressis. H. F. 201 .

A. $60-100$ h. Fl. 3-4. In sylvis humidis, ad vias etc. frequens.

Dum haec scribo $a b$ amico Schultz afferuntur mihi $P$. tremulae rani, ab uno eodem. que arbore decerpti, in quibus conspiciuntur amenta ㅇ discreta, flores $\sigma^{\top}$. et $\$$ in unum amentum conjuncti et flores $\Varangle$ in aliis amen. tis. In arbore autem, unde rami demti erant, amenta mascula, quae decidua esse solent, ad. huc haerebant. 
4. P. nigra: fol. deltoideis acuminat. serrat. utrinque glabris longitudine latitudinem superantibus, ramul. juniorib. glabris. H. F. 201,

A. $60-80^{\circ}$ t. Rami patentes. Fl. 4. Ad vias frequentissima.

5. P. dilatata (Aiton): fol. deltoideis acuminat. serrat. utrinque glabr. latitudine longitudinem su. perantibus. H. F. 201.

A. 20-80' h. Rami coarctati. Fl. 4-5. Ad vias frequentissima incola Italiae. Omnes fere arbores sunt $\sigma^{7}$.

6. P. monilifera (Aiton): fol. subcordat. basi glandulos. acuminat, serratis, serratur. hamat, cartila. gineis brevissime ciliatis utraque pagina glabris, nervis patentibus, petiol, compressis, ram. teretibus. H. F. $\delta 05$.

A. 70-80' 万. Fl. 4-5. Arbor Canadensis hinc inde cultus. Thierg. Hallsche Thor etc.

ORDO VI. ENNEANDRIA. 399. MERCURIALIS.

Flor. $\sigma^{7}$ et . . Cal.3-partitus. Cor. o. Stam.9-10. Antherae globosae didymae. Flor. ㅇ. Cal. et Cor. maris. Styli 2, Caps. 2.cocca 2-localaris, locul. 1-spermis.

1. $M$. perennis: rad. repente, caule simplicissimo, fol, scabris, L. WV. 1V. 809 . 
A. $\frac{7}{2}-1 \cdot 2$ \%ol. petiolata opposita orata acuminata serrata. Fl. 5. Flor. axillares. Ia sylvis frondosis. Fasanerie W.

2. M. annua: rad. fibrosa, caule herbaceo brs. chiato, fol. oblong. glabris, flor. $\sigma^{\top}$ spicatis. I. W. IV. 810 .

A. $1-1 \frac{1}{2}^{\prime} \odot$. Fol. serrata. Fl. 7-8. Flor. 9 axillares $v_{0}$ geminati. In agris, ad ruderata eta Beim Invalidenhause. WV.

400. IIYDROCIIAPS.

Flor. o'. Spatha 2-phylla. Cal.3-fidus. Cor.3-petala. Filam. plurima. Styli 3 imperfecti in me. dio. Flor. O Cal. et Cor. maris. Styli 6. Caps. 6-locul. polysperma infera.

1. H. Morsus ranae. L. IV. IV. 812.

Caul. teretes sub aqua fuitantes et repentes 24. Fol. natantia cordata orbiculata. FI. 7,8 . Pedunculi axillares. Cor. albae. In aquis stagnantibus frequens.

\section{ORDO VII. DODECANDRIA.}

401. STRATIOTES.

Flor. O'. Spatha 2-phylla. Cal. 3-partitus. Cor. 3-petala. Stam. in ఫ̛ 20 omnia fertilia, in $\sigma^{7}$ 9-12 antherifera, reliqua antheris destituta (Neciaria). Flor. ․ Spatha Cal, et Cor, maris. 
Styli 6 bipartiti. Bacca inf. G-locularis, locul. polyspermis, sem. parietalibus.

1. S. aloides: fol. lineari-lanceolat. carinat. aculeato-denticulatis. L. W. IV. 820 .

Caul. stoloniferi 24. Fol, erecto-patentia, cas naliculata, acuminata, margine serrata. Fl.6,7. Cor. alba. In aquis stagnantibus frequens.

\section{ORDO I. MONADELPHIA.}

\section{JUNTPEUS.}

Flor. ठ7. Amentum ovat. imbricat. baseos squamis sterilibus. Cal. o. Cor. o. Stam. in flore terminali 3 cum filament. brevibus, in squamuli Hore $2-6$ sine flamentis. Flor. ㅇ․ Amentum ovat. imbricatum, squam. sterilibus. Cal. o. Cor. o. Germ. 1 in Rore uno alterove termiuali. Styl. 3. Bacca c. amenti squamis coalita gibba 1--3.sperma.

1. F. communis: fol. tern. patentib. canaliculatolinearibus, apice attenuat, spinescenti-acuminatis, bacca longioribus. H. F. 205.

A. 10-15' (apud nos) T. F1. 5. Baccae atroviolaceae. In sylvis acerosis hinc inde. Teg. Hasenh. etc. Off.

$$
\text { 403. TAXUS. }
$$

Flor. ఫౌ. Cal. 0. (uisi gemma squamae). Cor. 0. 
Stam. plurima, anther. dentat. peltatis. Flor. 9 Cal. o (nisi gemma squamae), Cor. o. Germ. 1. Styl. o. Nux receptac, dilatato cum gemmae squamis baccato cincta.

1. T. baccata: trunco arboreo, ram. patenti-divergentibus, fol. distich. linearib. mucronatis, margine revolutis subtus bruneo-ligurinis opac. plerumque obsolete carinatis, carin. opacis, florib. solitar. confertis, receptac. $\sigma^{\top}$ globoso. H. F. 206. A. 20-30' 7. Fl. $3-4$. In sylvis et in ne. moribus hinc inde culta. Thierg.

\section{C1. XXIII. CRYPTOGA MIA. FILICES.} 404. EQ U I S E T U M.

Receprac. peltata multangula subtus indusiata, in racemum scrobuliformem disposita. Indusia 4-8 saccata latere interiore longitudinaliter dehiscentes. Stam. 4. Germen 1 subglobo. sum. Stylus o. Semen 1 (?). (Caulis aplyyllus verticillatim ramosus vaginatus.).

I. E. arvense: caulib. sterilib. plerumque simpliciter ramosis scabriusculis, ramis tetragonis, caulib. Exuctificantib. simplicibus, vaginis subcylin- 
draceis inciso-dentatis, dentib. acuminatis. II. A. VIII. 46.

$\alpha$. procumbens. $\beta$. erectum. A. $\frac{r}{2}-1^{\prime} 2 \%$. Fl. 4, 5. In agris sterilibus et fertilibus frequens.

2. E. sylvaticum : caulib. sterilib. fructificantibusque duplicato-ramosis, ramis scabriuscul. deflexis tetragonis, ramul. subtriquetris. L. W.V.3.

A. 1-1 $\frac{T}{2}{ }^{\prime}$ 4. Rad. repens. Fr. 4-6. In solo humidiusculo, ad fossas, in sylvis. Teg. Papenb. Charlottb. etc.

3. E. umbrosum (Meycr): caulib. sterilib. simpliciter ramosis, ram. scabriuscul. triquetris, fructificantibus demum ramosis. L. W. V. 3.

Antecedenti similis, 24. Fr. 6, 7. In sylvis subhumidis. Charlottb. Schlolsgarten. Strempel.

4. E. limosum: caulib. subramosis, ram, subduoden. pentagon. İaevib. glabris, spica terminali. I. W. V. 4 .

«. nudum. $\beta$. ramosum. A. $1-2,2$, FI. 5, 6. In paludibus turfosis et fossis frequens.

5. E. palustre: caulib. simpliciter ramosis glabris sulcatis, ram. 5-gon. apice spiciferis. L.W. V. 5 .

A. $1-1 \frac{T^{\prime}}{2} 2$. Fr. 5, 6. In solo humido es in aqua frequens.

6. E. hicmale: caulib, simplicib, erect, scabes- 
rim. apice spiciferis, vagin. discoloribus basi apiceque sphacelatis, dentib. aristatis omnino caducis. L. W. V. 8 .

a. vulgare. B. brachiatum. A. 2-3' 4 . Rad. repens. Fr. 5, 6. In collibus siccis, ad margines paludum et in pratis. Kreutz-Berg. Jungfernl. etc.

$$
\text { 405. IrCONODIUM. }
$$

Caps. reniformes uniloculares bivalves polyspermae. Sem. minutissima pulveriformia. * Axillis foliorum fructiferis (Selago).

1. L. Selago: fol. spars. octofariis lineari-lanceo. lat. acuminat. integerrimis imbricato-patul. rigidis, caule dichotomo erecto, ram. fastigiatis. I. V. V. 49 .

A. $3^{\prime \prime}-\frac{T}{2}$ ' 24. Caul. adscendens v. erectus. Fol. 8-1n.faria. Fr. 6-s. In nemoribus ad arbores rarius. Grunw.

* * Spicis terminalibus fructiferis.

2. I. clavatum: caule repente, ramis adscendentibus, fol. spars. enerv. incurvato-setigeris, spicis geminat. cylindraceis pedunculatis, bracteis ovat. actuminat, eroso-denticulatis. L. W. V. 16. H, A. VIII. 47.

Caulis repens 24 . Fr. 7 , s. Spicae 2-4. In pinetis frequens. Off.

3. L. inundatum: caule subramoso repente, ramis 
simplicib. solitar. erect. apice monostachyis, fol. linearib. spars. acut. integerrim. supra curvis, spica sessili foliosa, L. W. V. 25.

A. 3-4" 24. Fr. 8, 9. Spicae brácteae maximae. In Iocis humidis ad paludes. Jungfernh. Grunw.

4. L. annotinum: caule repente, ram. adscendentibus basi bipartitis, ramul. simplicibus, fol. 5.fariis lineari-lanceolat. mucronatis, apice serrulatis patentib. ad incrementa annua contractis, spic, so• litar. sessilib. terminalibus. L. W. V. 23 .

Fr. 7, S. In sylvis muscosis hinc inde. Teg. Jungfl. Cöpn. Heide.

E. L. complanatum: caule erecto, ram. alternatim dichotomis, fol. bifariis connat. apice patentibus, superficialib. solitariis adpressis, peduncul. 4-fid. 4-stachyis, spicis teretibus eylindraceis, $L$. W. V. 19 .

Rami alato-foliosi. 24. Fr. 7, 8. In sylvis acerosis hinc inde. Jungfh. Spand. Teg. etc.

$$
\text { 406. орнioclossum. }
$$

Capsulae nudae in spicam articulatam disticham connatae 1-loculares transverse dehiscentes 2-valves.

1. O. vulgatum: spica caulina, fronde ovata ob. tusa arcte reticulata, L..W. V.58. H. A. VI, 48. 
A. $2-2 \frac{1}{2} \cdot 2$. Rad. fibrosa lateraliter prolem sequentis anni profert. In pratis humidis apertis et umbrosis. Thierg. Frankf. Th. Fried. richsf, etc.

407. BOTR ICHIUM.

Capsulae subglobosae rachidi racemi compositi adnatae discretie nudae uniloculares, valvul. binis postice connexis, transverse dehiscentes. 1. B. Lunaria (Swartz): scapo superne unifrondoso, fronde subsolitaria pinnata et bipinnata, foliol. integcrim. crenat. incisis dentat. pinnatifidisque.

Osmunda Lunaria.

a. vulgare. $\beta$. rutaceum. Strempel. Filic. p. 21. Planta variabilis, 24 . Fr. 5, 6. In syl$\checkmark$ is graminosis siccioribus, in collibus apricis. Jung fh. Grunw. Tafsd. Kalkb. etc.

$$
\text { 40.8. OS MIU N DA. }
$$

Caps. subglobosae pedicellatae striatae semi.bivalves paniculatae frondem contractam tegentes. Indusium o.

1. O. regalis: frondib. bipinnatis, foliol. lanceolat. subintegerrim. basi inferiore subauriculatis, inferiorib. oppositis, panicula bipinnata fructif. cante in apice frondis. L. W. V.97.

A. $2-4^{\prime} 2$ In pratis et nemoribus subhumidis hinc inde, Charlottb. Teg. Strempel. Papenb. 


\section{POI I PODIUM.}

Caps. gyratae irregulariter dehiscentes. Sori dorsales subrotundi sparsi. Indusia o.

r. P. vulgare: frondib. profunde pinnatifidis, fo. liol. lineari-lanceolat. obtus. crenulat. approximaris, superiorib. sensim minoribus, soris solitariis, caudice paleaceo. L. W. V. 172.

A. $2 "-\frac{T}{2} " 24$. Plad. repens squamis paleaceis obsita. Fr. 6, 7. Ad vias, in declivibus, ad arbores frequens, Pichelswerder. Grunw. etc. :

2. P. Phegopteris: frondib. bipinnatifidis, pinn. duab, infim. deflexis, foliol. lineari.lanceolat. ob. tus. integerrim, ciliatis, infim, adnato-decurreuti. bus cum opposit. rhombum fere formantibus, venis Lirtis, soris solitar, marginalibus. L. W. V. 199.

A. $\frac{1}{2}-1 \cdot 2 \%$. Rad. repens. Frons pyramida. lis simplex. Fr. 7,8 . In nenoribus umbrosis humidis raius. Teg.

3. P. Diyopteris: frondib. ternat. bipinnat. patulo-deflexis, foliol. obtus. subintegerrimis, soris marginalibus, radice filiformi. L. IV. V. 209.

A. $3^{\prime \prime}-\frac{\pi}{2} 2 \%$. Rad.repens. Fr. 7 , 8. In sylvis subhumid, hincinde. Grunw. Teg. Cöpn. Heide.

\section{A S1'IDIUM.}

Caps. gyratae irregulariter dehiscentes. Sori dor. sales subrotundi sparsi. Indusium umbilicatum v. uno latere dehiscens. 
* Indusium orbiculare peltatum stipitatum in medio soro insertum undique liberum. (Aspidium R. Brown.)

1. A. Oreopteris (Swartz): frondib. pinnatis, foliol. lanceolat. glabris subtus resinoso-glandulos. pinnatifidis, laciniis lanceolat. obtus. integerrimis, infimis sublongioribus, sor. marginalibus. L. W. V. 247.

A. 1-3. 24. Fr. 7, 8. In sylvis subhumidis rarius. Teg. IV. Jungfh. Reliberge Strempel.

2. A. aculeatum (Swartz): frondib. bipinnatis, foliol. ovat. subfalcat. acut. mucronato-serratis, basi superiore truncatis, inferiore abbreviato-cuneatis, superiorib. fructificantibus stipite rachique paleaceis. L. W. V. 258.

A. 1-2, 4. $\mathrm{Fr}, 6-8$. In montosis umbro. sis sylvarum frondosarum. Pritzhagen. Strem. pel. 29.

* Indusium reniforme, sinu affixum, marginibus liberis Nephrodium $R$. Brown).

3. A. spinulosum: frond bipinnatis, foliol. oblong. decurrentib. inciso.dentatis, laciniis mucro. nato-serratis, stipite paleaceo, indusiis glandulosis. L. W. V. 262.

Nephrodium spinulosum. Strempel. 30.

F. B.

A. 1-2, 4. Rad, ex bulbis quasi compo. 
sita. Fr, 6, 7, 8. In nemorib. humidiusculis frequens. Jungfh. Tesp. etc.

4. A. Filix mas: frondib, bipinnatis, foliol. oblong. obtus. serratis, serratur. muticis, soris cristae mediae approximatis, stipite rachibusque paleaceis. L. WV. V. 259.

Nephrodium Filix mas. Strcmp. 3o.

A. $1-3 \cdot 2$. Rad. crassa paleacea, fibrillis subrufis. Fr. $6-8$. In nemoribus humidiusculis frequens. Off.

5. A. cristatum: frondib. pinnatis, foliol, subcordato-oblong. pinnatifidis, laciniis oblong. obtus. dentato-serratis, stipite paleaceo. L. VV. V. 252.

Nephrodium cristatum. Stremp. 31 .

A. 1-2' 4. Rad crassiuscula subpaleacea. Fr. 7, 8. In sylvis humidis turfosis. Grunw. an einer Stelle.

6. A. Thelypteris: frondib. pinnatis, foliol. lineari.lanceolat pinuatifid. glabris, laciniis ovat. acut. integerrimis, soris marginalib. contiguis demum confluentibus. L. W. V. 249.

Nephrodium Thelypteris. Stremp. 32.

A. $1-3^{\prime} 4$. Foliola alterna subtriangularia. Fr, 6. In locis humidis frequentissimum.

* Indusium oblongum altero latere venae lateraliter affixum, altero liberum (Athyrium Roth). 
7. A Filix femina: frondib. bipinnatis, foliol. oblongo-inciso-serratis, serratur. bi- sive triden. tat. acutiusculis, sor. oblong. rectis. L. W. V. 276. A. $1-4^{\prime}$ 4. Rad. omnino nigra paleacea. Fr. 6-8. In sylvis undique frequens.

8. A. fragile: frondib. bipinnatis, foliol oblong. obtus. inciso-serratis, serratur. obtus, denticulatis, rachide alata: L. W. V. 280.

A. $\frac{r}{4}-1,2$. In fossis et viis cavis rarius. Bei Schönhausen.

\section{A SPIENIUN.}

Caps. gyratae irregulariter dehiscentes. Sori dor. sales lineares transversales sparsi. Indusia e ve. nis lateralib. orta costam versus dehiscentia.

1. A. Trichomanes: frondib. pinatis, pinn. sub. rotundo-oblong. obtus. crenat. basi truncato-cuneatis, stipite discolore. L. IV. V. 331.

A. $2-6$ " 4 . Stipites atro-purpureo nigricantes, nitentes. Fr. 7, 8. In muris et fossis. muscosis rarius, Charlottb. Sclilofsgart. Jungfernh. S.

2. A. Ruta muraria: frondibus basi bipinnatis, apice simpliciter pinnatis, foliol rhombeo-oblong. obtusis, apice obtuse denticulatis. L.W.V.341.

A. 2-4" 24. Fr, 6-8. In muris vetustiori-

bus. Giefshaus. Maner v. Spandan etc.

3. A. Adiantum nigrum: frondibus bipinnatis; pinnis oblongo-lanceolat, acutis, foliol. oblong. 
pinnatifido-incisis, laciniis apice dentatis, soris de. mum contluentibus. L. W. V. 346 .

A. $\frac{3}{4}-1 \cdot 24$. Rad. fibrosa. Stipites prinum virides demum purpurascenti-fuscescentes. Fr. 7, 8. In locis montosis. B. Potsdam. Strempel. 412 PTERIS.

Caps. gyratae irregulariter dehiscentes, Sori lineares marginales continui. Indusium \& margine frondis inflexum interius deliscens.

I. $P$ a aquilina: ram. bipinnatis, foliol linearilanceolatis, superiorib. indivisis, inferiorib. pinnatifidis, laciniis oblong. obtusis, L. W. V. 402.

A. $\frac{1}{2}-3^{\prime} 4$. Fr. $6-8$. In agris, sylvis undique frequens.

413. BLECH UM.

Caps. gyratae irregulariter dehiscentes. Sori lineares longitudinales continui costae frondis utrinque paralleli. Indusium superficiarum continuum interius dehiscens.

1. B. boreale (Swartz): frondib. sterilib pinnatifidis, laciniis lanceolat. obtusinsculis parallelis, fructiferis pinnatis, pinnis linearibus acuminatis. L. W. V. 408 .

A. $\frac{\pi}{4}-1$ 24. Fr. 7,8. In locis humidis et subhumidis sylvarum. Jungfh. Strempel. Teg. 415. PILULARIA.

Fructus radicales. Indusia imbricata connata capsulam 4-locularem 4-valvem mentientia. Sem. numerosa. 
1. P. globulifera. L. W. V. 535.

A. $2 \frac{1}{2}-3 \cdot 24$. Caulis ramostus repens. Fol. fasciculata linearia subulata. Vernatio circina. lis. Fr. 7, 8. In lacubus. Temph.

416. SA L VINIA.

Fructus radicales. Indusia imbricata connata cap. sulam 1-locularem mentientia. Sem. recepta. culo centrali inserta.

1. $S$. natans: fol. ellipticis obtus. supra fascicu. lato-setosis, petiol. pilosis, fructib. subsessilib. ag. gregatis. L. W. V. 536 .

Fr. 8 Natans inter arundinem et trabes. Bei Pichelswerder. Stadt n.d. Oberbaum hin. Schlolsg. z. Charlottenb, sonst häufig. S.

\section{A L G A E. (?) \\ 417. CHARA.}

Globuli epigyni (lateritio-croceí) in laminas 3-4 dehiscentes, fila spiralia conglomerata emitten. tentes. Nucula subturbinata sessilis spiraliter striata, involucro diaphano apice obsolete 5fido corticata 1-locularis polysperma non dehiscers. Sem. minutissima sphaerica argentea. (Caulis verticillatim ramosus articulatus.)

1. C. flexilis: canlib. utricnlat. diaphan. Alaccid. glaberrim. ramosissimis, ramis opposit. patentibus, verticillorum ramulis $6-8$ inarticulatis aphyllis 
apice bi-ter-furcat. simplicibusque obtusis, nucui. obracteat. lateralibus. Wallr. Ann. bot. p. 176.

In aquis lacuum et fossarum passim. 24 . Jungfh. Teg. etc.

2. C. vulgaris: caulib. ramos teretib. scabriuscul. verticillorumq. ramul. 8.nis linearib. apice aphy!! inarticulat, obtusis, bracteis 4 -nis inaequalibus binis longissim. nuculas duplo excedentibus, reliquis inferiorib. illas aequantibus. Wallr. Anu. bot. 179.

In fossis et paludibus frequentissima. 4.

3. C. pulchella: caulib. gracilib glaberrin. ramos. pellucidis, ramul. S-nis lincari-subulat. articulat. aequalib. mucronatis, bracteis quatern, nucula ovali brevioribus. WVallr. Ann. bot. 184.

In fossis quietis. Friedrf. S. II. 46 .

4. C. hispida: caulib. hispid. ramos. inferne demum inermib. contorto-sulcat. suberosis superne incrassat. aculeatis, aculeis dens. setaceis reversis, verticillor. ramul. 8 -nis 6-articnlat patentibus, beticteis inaequalib. 4-nis nuculas ovatas subaequantibus. Wallr, Ann. bot. 184 .

Chara tementosa. Kt.l. 240.

In aquis stagnantibus. 2\%. Tafsd. Treptow. Spandau etc.

5. C latifolia: caulib. scabriuscul. rumosis, ramul. verticillat. subsenis articulat. complanatis, mucrone terminatis, nucul. nudis, bractcis o, Kth, $24 \mathrm{~L}$ In lacubus prope ripas. 4 . Teg. 


\section{A D DITAMENTA.}

\section{Cl. IV.}

тंтAMLOETON. (pag. 68.)

Schlechtendal II. 245 hoc medo notas spee cificas Potamogetonis Zosterifolii, compressi et pusilli emendandos esse censet.

8. P. Zosterifolius (Schumacher): caule complae nato-ancipiti, fol. linearibus nultinerviis basi eglandulosis.

9. P. compressus: caule compresso, fol. linearib. 3--5-nerv. basi biglandulosis.

10. P. pusillus : canle compressiusculo tereti, fol. setaceo-linearib. 3-nerv. basi biglandulosis.

\section{Cl. $Y$.}

pulmonaria. (pag. 7i.?

2. P. angustifolia: fol. pubescenti-hirsut. lanceolatis, radicalib. petiolatis, canlin. sessilib. semi. amplexicaulib. Lehni. A. 27j.

A. $\frac{\pi}{2}-\frac{3}{4} ; 2$. Fl. 4, 5. Cor. coeruleo-purpurascens. Cal. profundius partitus, laciniis angustioribus. In montibus calcareie. Fïdersd, Cruse. S. II. 246 .

$$
\text { AxAGALIIs. (pag. 76.) }
$$

Attentos esse Botanicos vult S. II. 246 de diso 
crimine specifico $A$. coeruleae et phoeniceae, dum eas diversas esse species dubitat.

\section{cuscuta. (pag. 100.)}

Num latet apud nos quoque IV eihii species C. epilinum?

Gentiana. (pag. 100.)

Delendum est secundum S. II. 247. nomen G. uliginosa (Willdenow) et ejus loco inserendum G. Amarella $L$.

BUPIEURUM.

Involucr. 5-phyllum umbellam subaequans. Fruct. ovati s. prismatici solidi, cost. 5 obtusiusculis, sutura excavata. (Fol. simplicia.)

1. B. falcatum: involucro universali sub-5-phyllo, partialib. 5-phyll. acutis, fol. radicalib. oblong. petiolatis, caulin. lanceolato-linearibus, caule flexuoso. L. R. S. VI. 396.

A. 1-2' 4. F1. 6, 7. Cor. Alavescens. In declivibus ripae ad lacus. Temph. Eysenhardt. S. II. 247.

SESELI. (pag. 104.)

2. S. annuum: caule erecto striato simpliciusculo, petiol. vaginantib. strict. foliisq. decomposit. erectis, foliol. linearib. 3-partit. cuspidatis, involucro partiali polyphyllo sublanceolato umbellis breviore, L, R. S, VI. 398 . 
A. $1-2^{\prime}, \sigma^{7}$. Fl. 6, 7. Cor. albida. In collilibus siccis herbosis, Scliulzend. Crome S. II. 2 \&S.

\section{XIII. \\ TIIIA. (pag. 188.)}

1. $T$. parvifolia addendum Synonymum $T$. micro. phylla. IV. Enum. 565.

2. T. vulgaris addendum Synony mum T.europaea.

3. $T$. pauciflora addendum Synonymum T. grandifolia. H off m.

NENUPHAR. (pag. 188.)

Synonym, addendum. Nymphaea lutea.

\section{Cl. XIII.}

CALEOPSIs, (pag. 209.)

Galeopsis Walterina Schlechtendal, addendum Synonymum $G$. pubescens Besser.

$$
\begin{gathered}
\text { Cl. XV. } \\
\text { MALVA. (pag. 243.) }
\end{gathered}
$$

In animadversione ad $M$. rotundifoliam facta pro s. $M$. neglecta etc. indigena sit: legendum est et $M$. neglecta Wallroth s. $M$. rotundifolia $L$. indi. genae sint.

Attamen, quum Cel Schl. observasse videatur utramque speciem, jam addere liceat notas earum. 1. $M$. rotundifolia: prostrata, fol. cordato-rotund. 
7-lobisqque duplicato-dentatis, cor. caljcem duplum longa, peduncul. subbin. demum declinatis, achaen. laevib. pubescentib. (disco majusculo). Reichenb. Icon. Cent. I. Dec. II. p. 20.

M. neglecta Wallroth.

2. M. borealis (Wallmann) prostrata, fol. cordatorotund. 7-lo-bisque subaequaliter dentatis, cor. calycem aequante, peduncul. subquatern. declinatis, achaen. marginat. reticulat. pubescentibus disco minimo. Reichenb. Icon. Cent. I, Dec. II. p. 21.

Malva pusilla Withering. 


\section{N D E X}

generum earumque specierum, quae sub aliis generibus latent.

Absinthium 287. vulgare 287.

Acer 177.

Achillea 356.

Achyrophorus 329.

Acinos 214.

vulgaris 214.

Acorus 152.

Adonis 231.

Adoxa 180.

Aegopodium $10 \%$.

Podagraria 10\%.

Aesculus 168.

Aethusa 128.

Agrimonia 207.

Agropyrum 5.3.

caninum 53.

repens 53 .

Agrostemma 201. Agrostis 30 .

lanceolata 26.

Aira 37.

aquatica 42.

caryophyllea 31.

cristata 39.

flexuosa 31.

glauca 39.

precex 31.

Ajuga 235 .

Alchemilla 70 .

Alisma 167.

Alliaria 282.

Allium 158.

Alnus 374.

Alopecurus 26 .

Alsine 162.

media 162.

Althaea 289.

Alyssum 266.

paniculatum 229. 
sativum 228.

Amaranthus 378 .

Anagallis 8.3.

Anchusa 78.

angustifolia 7.3 .

Andromeda 186.

Anenione 230.

patens 195.

pratensis $\mathbf{1 9 5 .}$

Pulsatilla 195.

vernalis $19 \%$

Anethum 107.

Foeniculum 107.

graveolens 115.

Angelica 142.

Antennaria $28 S$.

dioica 288.

Anthemis 355 .

Anthericun 155 .

Anthoxanthum 15.

Anthriscus 135.

Anthyllis 298.

Anthirhinum 260 .

arvinse 221.

Elatine 221.

Linaria 221.

minus 222.

A pargia 323.

Apium 125.

Aquilegia 226.

Arabis 279 .

Axbutus 187.
Arctinm 333.

Arenaria 197.

tenuifolia 163 .

Aristolochia $36 \mathrm{~s}$.

Armeria 149.

Arnoseris 331 .

Arrhenaterum 49.

Artemisia 342 .

Arundo 3I.

festucacea 40.

Phragmites 47.

Asarum 204. Asclepias 101.

Vincetoxicum 101.

Asparagus 153.

Asperngo 77.

Asperula 63.'

Aspidium 410.

Asplenium 412 .

Asprella 21.

oryzoides 21.

Aster 347 .

Astragulus 310.

Athamantha 113.

Cervaria 113.

Oreoselinum 114.

Athyrium 354.

Atriplex 111.

Avena 33 .

Ballota 245 .

Barbarea 277. 
Bellis 351.

lierberis 151 .

Berteroa 265.

Betonica 243 .

Letula 383 .

Bidens 338.

Blechnum 413 .

Botrychium 4n7.

Brachypodium 51.

Brassica 284.

Briza 42.

Bromus 35. erectus 37. giganteus 37. gracilis 52 . inermis 38 . pinnatus 52. pratensis 37 .

Bupleurnm 418. falcatum 360 .

Butomus 182.

Calendula 359 .

Calla 170.

Callitriche 2.

Calluna 175.

Caltha 234.

Calystegia 84.

sepium 84.

Camelina 272.

Campanula 102.

Cannabis 396 .
Capsella 270.

Cardamine 250.

Carduus 335.

Carex 372.

Carlina 337.

Carpinus 387.

Carum 122.

Catabrosa 42.

Caucalis 109.

Anthriscus 109.

arvensis 109.

scandicina 111.

Caulinia 335.

Centaurea 358.

Centunculus 68 .

Cerastiun 199.

Ceratophyllum 379.

Chaeropliyllum 134.

bulbosum 110.

temulum 110.

Chara 417 .

Chelidonium 218.

Chenopodium 1 ro.

Chilochloa 23.

Boehmeri 23.

Chimophila 153. corymbosa I53.

Chironia 78 .

Centaurium 78 .

inaperta 79 .

Chondrilla 320.

Chrysanthemum 352 . 
inodorum 298.

Chrysocoma 340.

Chrysosplenium 188 .

Cicer 305.

Cichorium 332 .

Cicuta 127.

Cineraria 349.

Circaea 11.

Cistus 190.

Helianthemum 190.

Cladium 14.

Clinopodium 248.

Cnicus 282.

acaulis 283.

lanccolatus $2 \$ 2$.

oleraceres 28.3 .

palistris 292.

Cnitium 123.

Cochlearia 268.

Coronopus 230 .

Colchicum 164 .

Comarum 216.

Conium 137.

Convallaria 154.

Convolyulus 93.

Cornus 65.

Coronilla 306.

Corrigiala 146.

Corydalis 291.

Corylus 385.

Corynephorus 39. canescens 39.
Crataegus I75.

monogyna 175.

Oxyacantha 175 .

Crepis 527.

Cucubalus 194.

Cuscuta 113.

epilinum 360 .

Cynanchum 115.

Cynog!ossum 76 .

Cynosurus 4.1.

Cyperus 20.

Dactylis 40.

Danthonia 29.

strigosa 29.

Datura 95.

Dancus 130 .

Delplinium 225.

Dechampsia $3 \mathrm{~g}$. caespitosa 38.

Dianthus 193.

Digitalis 262 .

Dipsacus 60.

Donax 39.

Draba 226.

verna 226.

Drosera 147 .

Echinochloa 47.

Echinospermum 72.

Lappula 72. 
Echium 82.

Elatine 18I.

Eleocharis 15.

Elymus 56.

Enodium 45.

Epilobium 172.

Epipactis 366.

Nidus avis 306. ovata 305.

Equisetum 404.

Erica $1-6$.

vulgaris 143 .

Erigeron 344 .

Eriophorum 23.

Erodium 287.

Erophila 267.

Ervum 304.

Lens 156 .

Eryngium 116.

Erysimum 283.

Alliaria 236.

Earbarea 232.

officinale 2.35.

Erythraea 88.

Eupatorium 339.

Euphorbia 224.

Euphrasia 255.

Evonymus 91.

Fagopyrum 14y. esculentum 149 . tataricum 149.
Fagus 386.

Fedia 1.7 .

Festuca 36 .

fluitans 42.

pinnata 52.

Ficaria 199.

ranunculoides 199.

Filago 289.

germanica 289.

montuna 290.

Fragaria 214.

Fraxinus 12.

Fumaria 292.

fabacea 24,4.

Galega 309.

Galeobdolon 24r.

Galeopsis 242.

TValterina 209.

ITalterina 361.

pubescens 361 .

Galingsogea 357.

Galium 64.

Genista 295.

Gentiana 11 ' Amarella 3 too. Centaurium 78. uliginosa 360 .

Geranium 288. cicutarium $239^{\circ}$

Geum 217.

Glechoma 239. 
Glyceria 4.3.

Gnaphalium 343 .

Goodyera 362 .

Gratiola 6 .

Gymnadenia 304.

Conopsea 304.

Gypsophila I9ı.

Habenaria 304. bifolia 304. Hedera 105.

Hedysarum 308 .

Helinnthemum 223.

Hepatica 196. triloba 196.

- Heracleum 140.

Herminium 30.5. Monorchis 305 .

Herniaria 112.

Hieracium 326 .

Hierochloe 51.

Hippuris 1.

Holcus 5 n.

avenaceus 49 .

borealis 50 .

bulbosus 49.

Holosteum 59.

Hordeum 57.

Hottonia $85^{\circ}$

Humalus 397.

Hydrocharis 400.

Hydrocotyle 118.
Hyoscyamus 9;. Hyoseris 279.

Hypericnm 315 .

Hy pochoeris 328 . maculata 278 .

radicala 278 .

Jasione 100.

Iberis 228.

nudicaulis 228.

Illecebrum 106.

Impatiens 90.

Inula 350.

Iris 18.

Isolepis 15.

acicularis 15 .

setacea 17.

supina 17.

Juncus 159.

campestris 132.

pilostis 132.

Juniperus 402 .

Koeleria 38 .

Lactuca 319.

Lamium 240.

Lappula 75 .

Lapsana 330.

pusilla 279.

Laserpitium 143.

Lathraea 257.

Lathyrus 302.

Ledum 184. 
Leersia 25。

Lemma 13.

Lepidium 275.

Leontodon 322.

autumnale 272.

hirtum 273.

hispidum 272.

Leonurus 247.

Galeobdolon 208.

Ligusticum 138.

Ligustrum 3 .

Liliam 157.

Limosella 263.

Linaria 259.

Linnaea 26\%.

Linum $15^{\circ}$.

Listera 364 .

Lithospermum 80.

Littorella 373.

Lolium 52.

Lonicera 104.

Lotus 313.

Luzula 160 .

Iychnis 200.

I_ycium 97.

Lycopodium 405.

Lycopsis 7.3.

arvensis 73.

Lycopus 9.

Lysimachia 86 .

Lythrum 205.

Malaxis 367 .

F. B.
Malva 290.

rotundifolia $36 \mathbf{r}$.

borealis 362 .

Marrubium 2.6 .

Matricaria 354.

Medicago 314.

Melampyrum 256.

Melica 46 .

coerulea 45.

Melilotus 311.

Mentha 238.

Menyanthes 87.

Mercurialis 399.

Mespilus 210.

Menm 1:6.

Milium 29.

Molinia 45.

Monotropa 183.

Montia 58 .

Morus 375.

Myagrum 238.

dentatum 229.

paniculatum 229.

sativum 228.

Myosotis 74 .

Lappula 72.

Myosurus 198.

Myriophyllųm 380 .

Myrrhis 133.

Najas 392.

Nardus 2, .

Nasturtium 276.

A a 
Nenupliar 221.

Neottia 363. repens 305.

Neottidium 365.

Nepeta 237.

Sephrodium 353.

Neslia 273.

Nigella 227.

Nymphaea 220. luteu 361 .

Ocnanthe 124.

Oenothera 171.

Onagra 14 \%.

biennis $\mathbf{3} 40$.

Ononis 296.

Onopordon 336.

Ophigglossum 406.

Ophrys 361.

Nidlis avis 306.

orata 306.

paludosa 308 .

spiralis 305.

Orchis 360 .

Origanum 249.

Ornithogalum 156.

Ornithopus 307.

Orobus 301.

Osmenada 408 .

Lunaria 351.

Oxalis 203.

Oxy coccus $174^{\circ}$

Panicum 48 .
Papaver 219。

Parietaria 71 .

Paris 179.

Parnassia 148.

Pastinaca 141 .

Pedicularis 258 .

Peplis 161.

Peucedanum 105.

Silaus 105.

Phalaris 28.

Phaseolus 299.

Phellandrium 106. aquaticum 106.

Phleum 27.

Phragmites 47.

Phyteuma 10I.

Picris 325.

Pilularia 415 .

Pimpinella 119.

Pinguicula 7 .

Pinus 389.

Pisum 306.

Plantago 67.

platanus 388 .

Poa 44.

aquatica $\$ 5$.

decumbens 32 .

distans 42.

fluitans 42.

serotina 14 .

Polycneinum 19.

Polygala 293. 
Polrgoninm 178.

Polyporium 409.

Populus 399.

Potamogeton 72.

Potentilla 215.

Poterium 382.

Prenanthes 321.

Primula 84 .

Prunella 252.

Prunus 206.

Psamma 27.

arenaria 27.

Pteris 412.

Pulmonaria 79.

angustifolia 359 .

Pulsatilla 229.

Pyrethrum 353.

Pyrola 185.

Pyrus 209.

Quercus 35千.

Radiola 120.

linoides 120.

Ranuriculus 232.

Raphanus 286.

Reseda 208.

Rhamnus 98 .

Rhinanthus 254 .

Rhynchospora 19. alba 19 . fusca 19.

Ribes 103.
Robinia 297.

Rosa 212.

Rubus 213.

Rumex 166 .

Sagina 73.

Sagittaria $3 \$ 1$.

Salix 393.

Salsola 109 .

Salvia 10.

Salvinia 416 .

Sambucus 145 .

Sanguiforba 69.

Sanicula 117.

Saponaria 192.

Satyrium 305.

repens 305.

Saxifraga 189.

Scabiosa 61.

Scandix 132.

Anthriscits 111 .

Cerefolium 111.

Schedonorus 37.

Scherardia 62.

Scheuchzeria 162.

Schoenus 22.

Schollera 143.

Orycoccus 143.

Scirpus 21.

Scleranthus 190.

Scorzonera 317.

Scrophularia 261.

A a 2 
Scutellaria 25 . S'ecale 55. Sedum 202. Selinum 13g. Carrifolia 116. Chabraei 104. palustre 108.

Sempervivum 169. rectorum 169. Senebiera 274. Senecio 346. Serapias 307. latifolia 307. longifolia 307. rubra 307 . Serratula 334 . arvensis 283. Seseli 120. annuum 360,

Setaria 48 . Silene 195. Sinapis 285 . Sison 121 . Sisymbrium 281. Alliaria 236. amphibium 232. arenosum 234. Nasturtium 231. sylvestre 231. Sium 136. Solanum 96. Solidago 348 .
Sonchus 318. palustris $26 \mathrm{~g}$. Sorbus 174. Sparganium 371. Spartium 294. Spergula 198 . Spinacia 395. Spiraea 211. Stachys 244 . Statice 119. Armeria 119. Stellaria 196. Stipa 32. Stratiotes 401 . Symphytum 81. Syntherisma 48 . Syringa 4.

Tanacetum 34r. Taxus 403.

Teesdalia $27 \mathrm{I}$. Teucrium 236. Thalictrum 228. Thésium 107. Thlaspi 269. bursa pastoris 227. campestre 230.

Thrincia 324 . Thuja 390. Thymus 250. Thysselinum 129. Tilia 221. 
europaea 361. microphylla 361. Tofieldia 165. Torilis 131.

Tormentilla 180. erecta 180. reptans 191. Tragopogon 316 . Trapa 66.

Trichodium 25. caninum 25. Trientalis $16 \mathrm{~g}$. Trifolium 312 . officinale 259 . vulgare $25 \mathrm{y}$. Triglochin 163. Triodia 33 . Triticum 54. Trollius 234 . Turritis 278. hirsuta 233.
Tussilago 345.

Typha 370.

Olmus 108 .

Urtica 376 .

Utricularia 8.

Vaccinium 173.

Oxycoccus 143.

Valeriana 16. dentata 12. olitoria 12.

Verbascum 92. Verbena 253. Veronica 5. Viburnum 144. Vicia 203.

Vinca 99 . Viola 89.

Viscum 394.

Xanthium 377. Zannichellia 369 . 
BEROLINI, LITTERIS C. A. W. SCIIMIDT. 



$$
29 \delta
$$




\section{New York Botanical Garden Library}

QK314.B7

Brandt, Johann Frie/Flora Berolinensis,

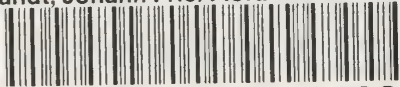

35185001208162 
